\title{
Validation of a Database of Formation Enthalpies and of Mid-level Model Chemistries
}

\author{
J. M. Simmie \\ Combustion Chemistry Centre \& School of Chemistry \\ National University of Ireland, Galway H91 TK33, Ireland \\ Tel. +353-91-492451 \\ Email: john.simmie@nuigalway.ie
}

J. N. Sheahan

School of Mathematics \& Statistics

National University of Ireland, Galway, H91 TK33, Ireland

Tel. +353-91-493103

Email: jerome.sheahan@nuigalway.ie 


\section{Contents}

$\begin{array}{llr}1 & \text { 1-propynyl-freqs.log } & 6\end{array}$

$\begin{array}{lll}2 & \text { 13Cyclopentadiene.log } & 7\end{array}$

$\begin{array}{llr}3 & \text { 1ButeneGauche.log } & 8\end{array}$

$\begin{array}{llr}4 & \text { 1Butyne.log } & 9\end{array}$

$\begin{array}{lll}5 & 1 \text { Cyclopropenyl.log } & 10\end{array}$

6 1HydroxyEthylGaucheAnti.log 11

$7 \quad$ 1HydroxyEthylGaucheSyn.log $\quad 12$

$\begin{array}{lll}8 & \text { 1Propynyl.log } & 13\end{array}$

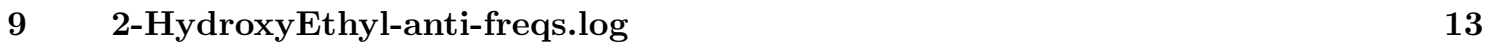

$10 \quad 2$-Hydroxyethyl-s-anti.log $\quad 14$

$11 \quad 2$-HydroxyEthyls-freqs.log $\quad 15$

$12 \quad 2$-Propynyl.log $\quad 17$

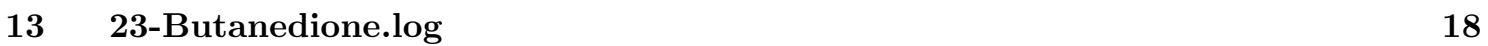

$\begin{array}{lll}14 & \text { 2HydroxyEthylGaucheAnti.log } & 19\end{array}$

$15 \quad 2$ HydroxyEthylGaucheSyn.log $\quad 20$

16 Allyl-freqs.log $\quad 21$

17 AminoMethyl.log $\quad 22$

18 Aminoxyl-H2NO-freqs.log 23

19 AmmoniaOxide.log 24

20 Benzene-freqs.log 25

21 CCNN-freqs.log $\quad 26$

22 ch2chchch3-cis.log $\quad 26$

23 ch2chchch3-trans.log 28

$\begin{array}{lll}24 & \text { ch2chchch3.log } & 29\end{array}$

25 Cis-2-butene.log 31

26 cis-HCOO.log 32

$27 \quad$ CNCN-freqs.log $\quad 32$

$28 \quad$ CNN-triplet-freqs.log $\quad 33$

$29 \quad$ CNNC-freqs.log 33

$30 \quad$ CPO-C2symm-freqs.log $\quad 34$ 
$33 \quad$ CyanicAcid.log

34 Cyanogen-anharmonic.log

35 Cyclobutanone-freqs.log

36 Cyclohexane-chair-D3d.log

38 Cyclohexane-chair-z-matrix.log

$39 \quad$ Cyclohexane-D3d-freqs.log

40 Cyclohexane-D3d.log

41 Cyclohexane-twist-boat.log

42 Cyclopropenyl-symm.log

43 Cyclopropenyl.log

$44 \quad$ Diazene-cis.log

45 Diazene-trans.log

46 Diazenyl.log

47 Diisocyanogen-mp2.log

48 Diisocyanogen.log

49 Dioxirane.log

50 Ethoxy-freqs-vibrot.log

51 Ethoxy-freqs.log

52 Ethylidene-singlet-loose.log

$53 \quad$ Ethylidene-singlet-scan.log

54 Ethylidene-singlet.log

56 Ethylidyne.log 60

$57 \quad$ Ethynol.log

60 Formyloxy.log 
62 Glyoxal-cis.log $\quad 64$

63 Glyoxal-trans.log $\quad 65$

64 HO2-freqs.log $\quad 65$

65 Hydrazine.log $\quad 66$

$\begin{array}{llr}66 & \text { Hydrogen-azide-HNNN.log } & 67\end{array}$

$\begin{array}{llr}67 & \text { Hydrogen-isocyanide.log } & 67\end{array}$

$\begin{array}{llr}68 & \text { Hydroxyamidogen-cis.log } & 68\end{array}$

$69 \quad$ Hydroxyamidogen-trans.log $\quad 69$

$\begin{array}{llr}70 & \text { Hydroxyformyl-cis.log } & 70\end{array}$

$\begin{array}{llr}71 & \text { Hydroxyformyl-trans.log } & 70\end{array}$

$\begin{array}{llr}72 & \text { Hydroxylamine-cis.log } & 71\end{array}$

$\begin{array}{llr}73 & \text { Hydroxylamine-trans.log } & 72\end{array}$

74 Hydroxymethylene-cis.log $r 2$

$\begin{array}{lll}75 & \text { Hydroxymethylene-g-triplet.log } & 73\end{array}$

76 Hydroxymethylene-trans.log $\quad 74$

$\begin{array}{llr}77 & \text { Iminomethyl-cis.log } & 74\end{array}$

$\begin{array}{llr}78 & \text { Iminomethyl-trans.log } & 75\end{array}$

$\begin{array}{llr}79 & \text { Isobutane-freqs.log } & 76\end{array}$

$\begin{array}{lll}80 & \text { IsocyanicAcid-HNCO.log } & 77\end{array}$

$\begin{array}{llr}81 & \text { Isocyanogen.log } & 78\end{array}$

$\begin{array}{llr}82 & \text { Isodiazine.log } & 79\end{array}$

$\begin{array}{llr}83 & \text { Isoformyl.log } & 79\end{array}$

84 IsofulminicAcid.log $\quad 80$

85 MethaneDiol-2.log $\quad 80$

86 Methanediol.log $\quad 81$

87 MethaneDiols-freqs.log $\quad 82$

88 Methylamidogen.log $\quad 85$

$89 \quad$ Methylenimine.log $\quad 86$

$\begin{array}{llr}90 & \text { Methylhydroperoxide.log } & 87\end{array}$

$91 \quad$ Methylperoxy.log $\quad 88$

$92 \quad$ N2H3.log $\quad 89$ 
$93 \quad$ NCCN-freqs.log $r$

94 NCN-freqs.log $\quad 90$

$95 \quad$ NitricAcid.log $\quad 91$

$96 \quad$ Nitrosobenzene.log $\quad 92$

$\begin{array}{llr}97 & \text { Nitrosylhydride.log } & 93\end{array}$

$98 \quad$ NitrousAcidCis.log $\quad 93$

$99 \quad$ NitrousAcidTrans.log $\quad 94$

100 NO-freqs.log $\quad 95$

101 nPrOO-freqs.log $\quad 95$

102 o-Benzyne.log $\quad 97$

103 OH-freqs.log $\quad 98$

104 Oxoethenyl.log $\quad 98$

105 Peroxynitrousacid-cis-cis.log $\quad 99$

106 Peroxynitrousacid-cis-perp.log $r$

107 Peroxynitrousacid-trans-perp.log 100

108 Toluene.log $r 101$

109 Trans-2-butene.log 103

110 Vinoxy-radical-freqs.log $\quad 104$

111 Vinylalcohol-anti.log $r 05$

112 Vinylalcohol-syn.log $\quad 105$

113 Vinylidene.log $\quad 106$ 


\section{Introduction}

A summary of Gaussian output files are presented below ${ }^{\S}$. The level of theory used for the geometry optimisation and frequency calculation is B3LYP/cc-pVTZ+d, appropriate for W2X and W3X-L. The scaled frequencies are not correctly represented and should be ignored because these Wx methods use different scaling factors for the purposes of computing the zero-point energy, the entropy and the enthalpy function. Note that for linear molecules, such as cyanogen, more frequencies are listed that are possible; this error is in the process of being corrected.

\section{1-propynyl-freqs.log}

\begin{tabular}{|c|c|c|c|c|c|}
\hline \multirow{2}{*}{$\begin{array}{l}\text { Center } \\
\text { Number }\end{array}$} & \multirow{2}{*}{$\begin{array}{l}\text { Atomic } \\
\text { Number }\end{array}$} & \multirow{2}{*}{$\begin{array}{c}\text { Atomic } \\
\text { Type }\end{array}$} & \multicolumn{3}{|c|}{ Coordinates (Angstroms) } \\
\hline & & & $\mathrm{X}$ & Y & Z \\
\hline 1 & 6 & 0 & 0.000000 & 0.351993 & 0.000000 \\
\hline 2 & 6 & 0 & -0.704933 & 1.381697 & 0.000000 \\
\hline 3 & 6 & 0 & 0.436089 & -1.031194 & 0.000000 \\
\hline 4 & 1 & 0 & 1.024011 & -1.261028 & 0.890367 \\
\hline 5 & 1 & 0 & -0.434954 & -1.692922 & 0.000000 \\
\hline 6 & 1 & 0 & 1.024011 & -1.261028 & -0.890367 \\
\hline
\end{tabular}

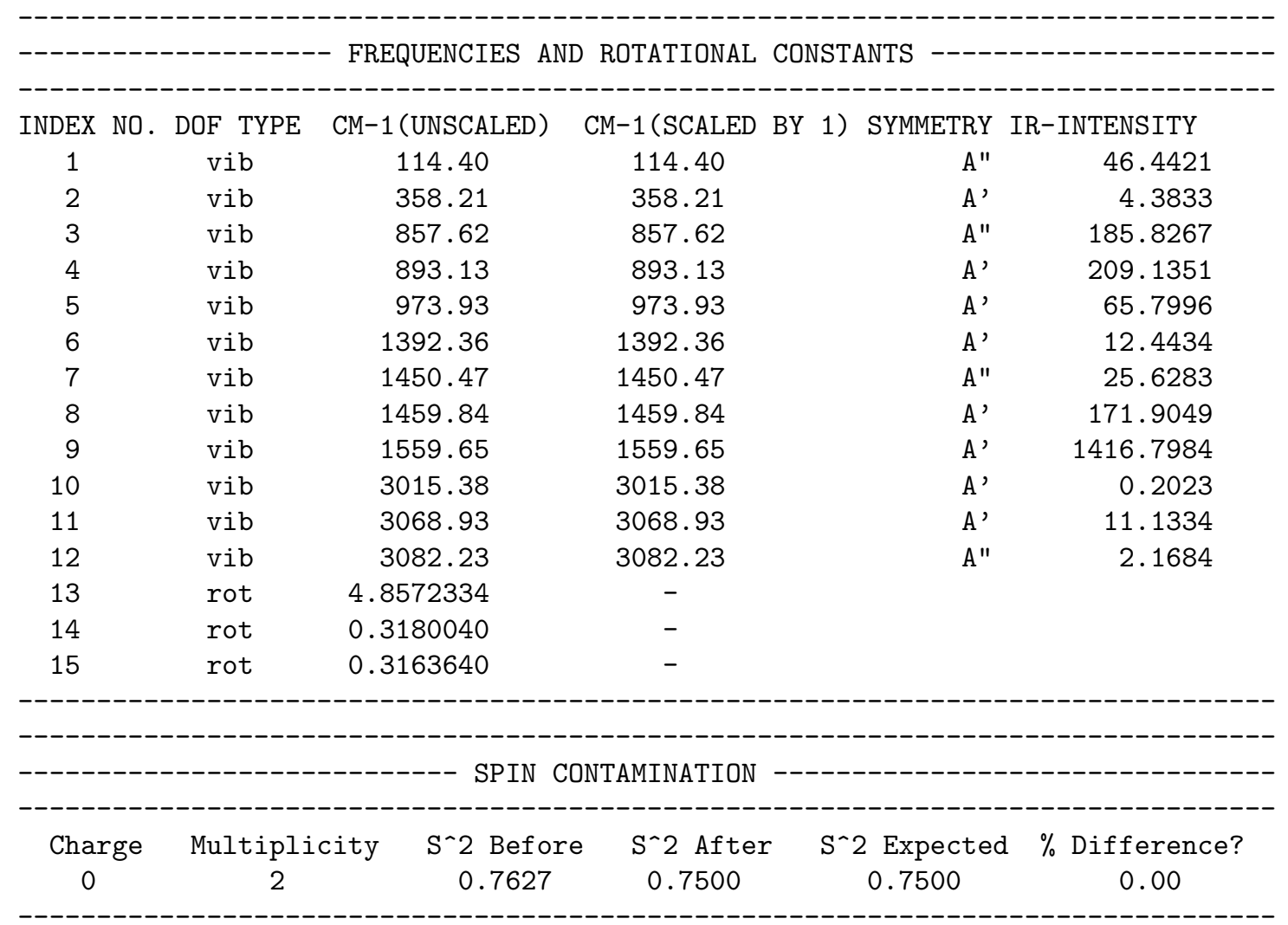

\footnotetext{
${ }^{\S}$ Application written and maintained by Dr. K. P. Somers; LATEXwrap by J. M. Simmie
} 


\section{13Cyclopentadiene.log}

\begin{tabular}{|c|c|c|c|c|c|}
\hline \multicolumn{6}{|c|}{$\begin{array}{r}-1 \\
-1--1\end{array}$} \\
\hline \multirow{2}{*}{$\begin{array}{l}\text { Center } \\
\text { Number }\end{array}$} & \multirow{2}{*}{$\begin{array}{l}\text { Atomic } \\
\text { Number }\end{array}$} & \multirow{2}{*}{$\begin{array}{l}\text { Atomic } \\
\text { Type }\end{array}$} & \multicolumn{3}{|c|}{ Coordinates (Angstroms) } \\
\hline & & & $\mathrm{X}$ & $\mathrm{Y}$ & $\mathrm{Z}$ \\
\hline 1 & 6 & 0 & 0.000000 & 0.732677 & -0.987410 \\
\hline 2 & 6 & 0 & 0.000000 & -0.732677 & -0.987410 \\
\hline 3 & 6 & 0 & 0.000000 & -1.176479 & 0.280380 \\
\hline 4 & 6 & 0 & 0.000000 & 0.000000 & 1.213169 \\
\hline 5 & 6 & 0 & 0.000000 & 1.176479 & 0.280380 \\
\hline 6 & 1 & 0 & 0.000000 & 1.344905 & -1.877401 \\
\hline 7 & 1 & 0 & 0.000000 & -1.344905 & -1.877401 \\
\hline 8 & 1 & 0 & 0.000000 & -2.205189 & 0.605999 \\
\hline 9 & 1 & 0 & -0.874514 & 0.000000 & 1.874072 \\
\hline 10 & 1 & 0 & 0.874514 & 0.000000 & 1.874072 \\
\hline 11 & 1 & 0 & 0.000000 & 2.205189 & 0.605999 \\
\hline
\end{tabular}

$\begin{array}{llll}\text { Rotational constants (GHZ): } & 8.5093089 & 8.2725298 & 4.3047798\end{array}$

FREQUENCIES AND ROTATIONAL CONSTANTS

INDEX NO. DOF TYPE CM-1(UNSCALED) CM-1(SCALED BY) SYMMETRY IR-INTENSITY

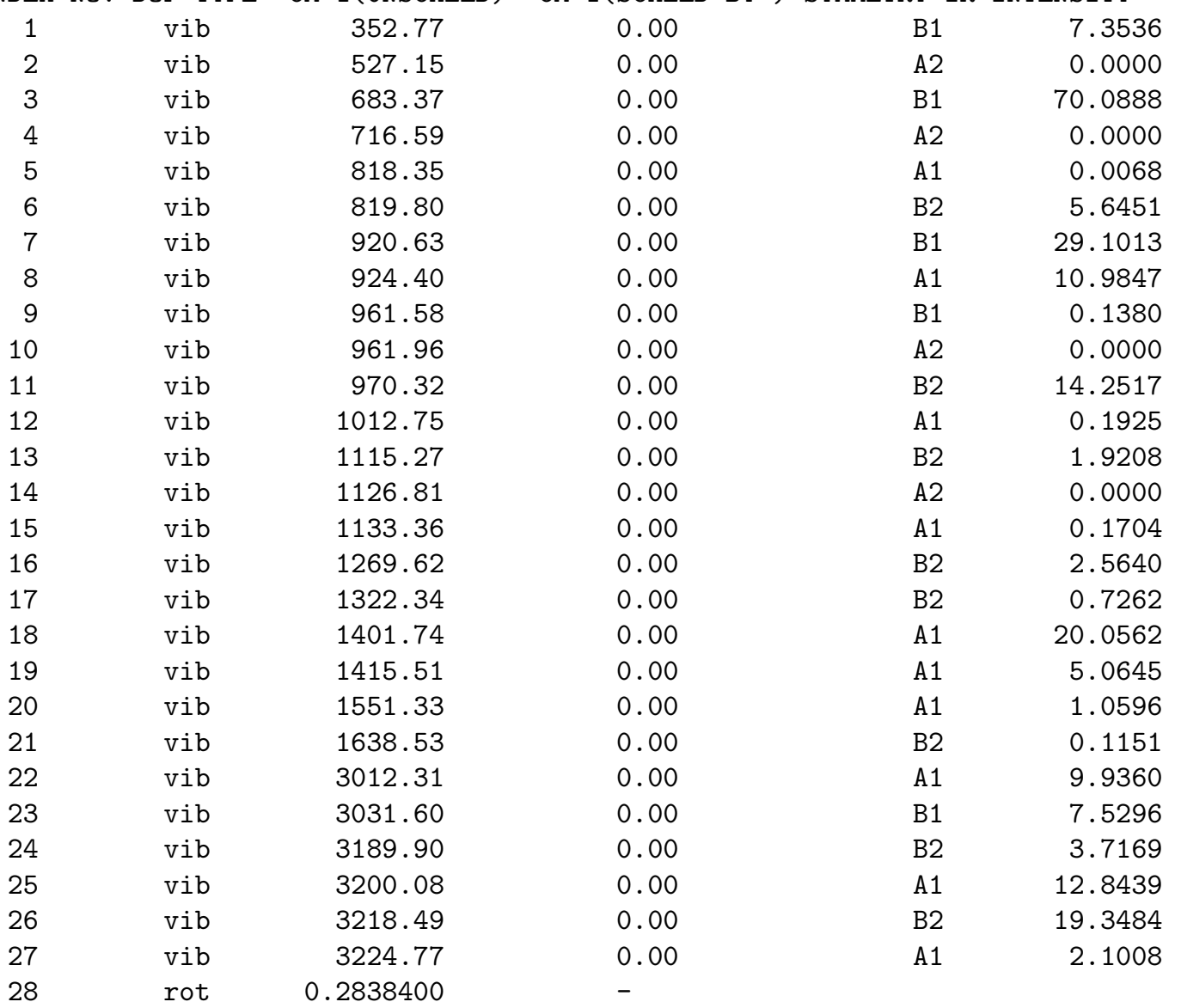




\section{1ButeneGauche.log}

\begin{tabular}{|c|c|c|c|c|c|}
\hline \multicolumn{6}{|c|}{ - } \\
\hline \multirow{2}{*}{$\begin{array}{l}\text { Center } \\
\text { Number }\end{array}$} & \multirow{2}{*}{$\begin{array}{l}\text { Atomic } \\
\text { Number }\end{array}$} & \multirow{2}{*}{$\begin{array}{c}\text { Atomic } \\
\text { Type }\end{array}$} & \multicolumn{3}{|c|}{ Coordinates (Angstroms) } \\
\hline & & & $\mathrm{X}$ & Y & Z \\
\hline 1 & 6 & 0 & -1.856001 & 0.018439 & -0.277479 \\
\hline 2 & 6 & 0 & -0.721957 & -0.295965 & 0.335156 \\
\hline 3 & 1 & 0 & -2.728835 & -0.618237 & -0.220547 \\
\hline 4 & 1 & 0 & -1.953158 & 0.928394 & -0.858276 \\
\hline 5 & 1 & 0 & -0.672297 & -1.221577 & 0.903725 \\
\hline 6 & 6 & 0 & 0.537140 & 0.517779 & 0.306836 \\
\hline 7 & 1 & 0 & 0.363563 & 1.438745 & -0.254192 \\
\hline 8 & 1 & 0 & 0.791753 & 0.817244 & 1.329112 \\
\hline 9 & 6 & 0 & 1.726001 & -0.244302 & -0.292215 \\
\hline 10 & 1 & 0 & 1.531192 & -0.517773 & -1.329938 \\
\hline 11 & 1 & 0 & 2.632682 & 0.361543 & -0.265239 \\
\hline 12 & 1 & 0 & 1.923998 & -1.164039 & 0.261566 \\
\hline
\end{tabular}

FREQUENCIES AND ROTATIONAL CONSTANTS

INDEX NO. DOF TYPE CM-1(UNSCALED) CM-1(SCALED BY ) SYMMETRY IR-INTENSITY

\begin{tabular}{|c|c|c|c|c|c|}
\hline 1 & vib & 108.50 & 0.00 & A & 0.0637 \\
\hline 2 & vib & 230.24 & 0.00 & A & 0.0264 \\
\hline 3 & vib & 316.38 & 0.00 & A & 0.5225 \\
\hline 4 & vib & 434.44 & 0.00 & A & 0.8055 \\
\hline 5 & vib & 655.89 & 0.00 & A & 9.8826 \\
\hline 6 & vib & 798.86 & 0.00 & A & 3.0146 \\
\hline 7 & vib & 857.88 & 0.00 & A & 2.2499 \\
\hline 8 & vib & 950.08 & 0.00 & A & 43.4929 \\
\hline 9 & vib & 991.89 & 0.00 & A & 1.9699 \\
\hline 10 & vib & 1020.01 & 0.00 & A & 2.3289 \\
\hline 11 & vib & 1036.59 & 0.00 & A & 9.7052 \\
\hline 12 & vib & 1096.16 & 0.00 & A & 3.8261 \\
\hline 13 & vib & 1202.26 & 0.00 & A & 0.3002 \\
\hline 14 & vib & 1292.59 & 0.00 & A & 0.1085 \\
\hline 15 & vib & 1325.09 & 0.00 & A & 1.1090 \\
\hline 16 & vib & 1348.04 & 0.00 & A & 2.3162 \\
\hline 17 & vib & 1411.43 & 0.00 & A & 1.6289 \\
\hline 18 & vib & 1459.56 & 0.00 & A & 1.9278 \\
\hline 19 & vib & 1484.55 & 0.00 & A & 3.6918 \\
\hline 20 & vib & 1499.11 & 0.00 & A & 5.9195 \\
\hline
\end{tabular}




$\begin{array}{rrrrrr}21 & \text { vib } & 1508.40 & 0.00 & \mathrm{~A} & 5.4988 \\ 22 & \text { vib } & 1708.06 & 0.00 & \mathrm{~A} & 12.6392 \\ 23 & \text { vib } & 3003.67 & 0.00 & \mathrm{~A} & 25.6064 \\ 24 & \text { vib } & 3025.83 & 0.00 & \mathrm{~A} & 31.0700 \\ 25 & \text { vib } & 3047.20 & 0.00 & \mathrm{~A} & 8.8292 \\ 26 & \text { vib } & 3086.46 & 0.00 & \mathrm{~A} & 35.9806 \\ 27 & \text { vib } & 3093.08 & 0.00 & \mathrm{~A} & 34.3688 \\ 28 & \text { vib } & 3112.43 & 0.00 & \mathrm{~A} & 30.9069 \\ 29 & \text { vib } & 3128.05 & 0.00 & \mathrm{~A} & 5.2642 \\ 30 & \text { vib } & 3208.57 & 0.00 & \mathrm{~A} & 18.4819 \\ 31 & \text { rot } & 0.7635906 & - & & \\ 32 & \text { rot } & 0.1381949 & - & & \\ 33 & \text { rot } & 0.1349972 & - & & \\ ------------------------\end{array}$

\section{1Butyne.log}

Charge $=0$ Multiplicity $=1$ Stoichiometry $\quad$ C4H6

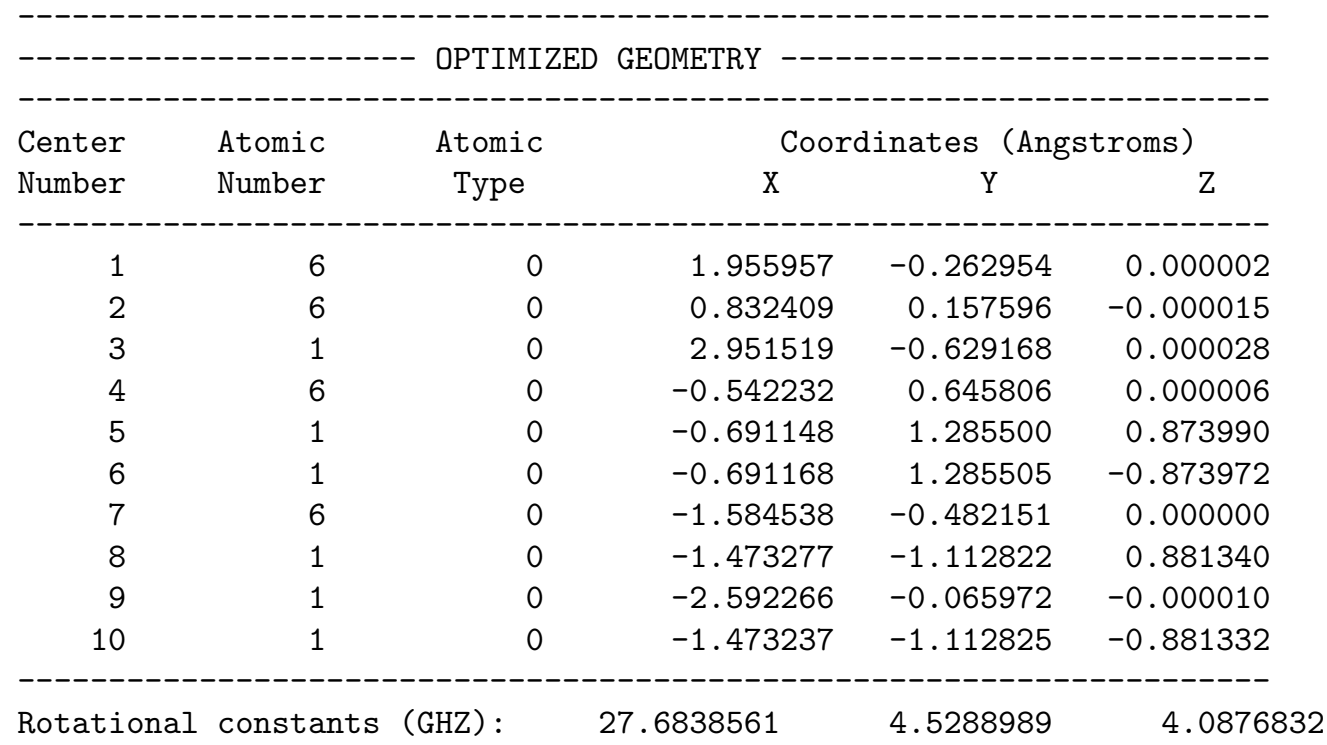

FREQUENCIES AND ROTATIONAL CONSTANTS

$\begin{array}{cccccr}\text { INDEX NO. DOF TYPE } & \text { CM-1 (UNSCALED) } & \text { CM-1 (SCALED BY ) } & \text { SYMMETRY } & \text { IR-INTENSITY } \\ 1 & \text { vib } & 201.73 & 0.00 & \mathrm{~A} & 1.3039 \\ 2 & \text { vib } & 219.66 & 0.00 & \mathrm{~A} & 0.5362 \\ 3 & \text { vib } & 361.55 & 0.00 & \mathrm{~A} & 7.0708 \\ 4 & \text { vib } & 521.87 & 0.00 & \mathrm{~A} & 7.7507 \\ 5 & \text { vib } & 662.32 & 0.00 & \mathrm{~A} & 47.2326 \\ 6 & \text { vib } & 670.82 & 0.00 & \mathrm{~A} & 44.7987 \\ 7 & \text { vib } & 788.31 & 0.00 & \mathrm{~A} & 1.1450 \\ 8 & \text { vib } & 845.58 & 0.00 & \mathrm{~A} & 0.0179 \\ 9 & \text { vib } & 1018.85 & 0.00 & \mathrm{~A} & 0.2876 \\ 10 & \text { vib } & 1089.82 & 0.00 & \mathrm{~A} & 2.8103 \\ 11 & \text { vib } & 1109.88 & 0.00 & \mathrm{~A} & 0.6648\end{array}$




$\begin{array}{rrrrrr}12 & \text { vib } & 1288.52 & 0.00 & \text { A } & 0.0000 \\ 13 & \text { vib } & 1349.31 & 0.00 & \text { A } & 8.5474 \\ 14 & \text { vib } & 1412.02 & 0.00 & \text { A } & 1.4662 \\ 15 & \text { vib } & 1478.90 & 0.00 & \text { A } & 3.1895 \\ 16 & \text { vib } & 1496.60 & 0.00 & \text { A } & 6.3953 \\ 17 & \text { vib } & 1506.90 & 0.00 & \text { A } & 3.6352 \\ 18 & \text { vib } & 2220.70 & 0.00 & \text { A } & 6.8631 \\ 19 & \text { vib } & 3021.13 & 0.00 & \text { A } & 16.9021 \\ 20 & \text { vib } & 3038.46 & 0.00 & \text { A } & 6.3323 \\ 21 & \text { vib } & 3044.43 & 0.00 & \text { A } & 28.8422 \\ 22 & \text { vib } & 3103.82 & 0.00 & \text { A } & 26.1223 \\ 23 & \text { vib } & 3109.71 & 0.00 & \text { A } & 63.1917 \\ 24 & \text { vib } & 3473.42 & 0.00 & & \\ 25 & \text { rot } & 0.9234340 & - & & \\ 26 & \text { rot } & 0.1510678 & - & & \end{array}$

\section{1Cyclopropenyl.log}

Charge $=0$ Multiplicity $=2$ Stoichiometry C3H3(2)

\begin{tabular}{|c|c|c|c|c|c|}
\hline \multirow{2}{*}{$\begin{array}{l}\text { Center } \\
\text { Number }\end{array}$} & \multirow{2}{*}{$\begin{array}{l}\text { Atomic } \\
\text { Number }\end{array}$} & \multirow{2}{*}{$\begin{array}{c}\text { Atomic } \\
\text { Type }\end{array}$} & \multicolumn{3}{|c|}{ Coordinates (Angstroms) } \\
\hline & & & $\mathrm{X}$ & $\mathrm{Y}$ & $\mathrm{Z}$ \\
\hline 1 & 6 & 0 & -0.490650 & -0.692115 & 0.000000 \\
\hline 2 & 6 & 0 & 0.000000 & 0.841899 & 0.000000 \\
\hline 3 & 6 & 0 & 0.867159 & -0.088710 & 0.000000 \\
\hline 4 & 1 & 0 & -0.433702 & 1.820325 & 0.000000 \\
\hline 5 & 1 & 0 & -0.912676 & -1.093383 & 0.916422 \\
\hline 6 & 1 & 0 & -0.912676 & -1.093383 & -0.916422 \\
\hline
\end{tabular}

FREQUENCIES AND ROTATIONAL CONSTANTS

INDEX NO. DOF TYPE CM-1(UNSCALED) CM-1(SCALED BY ) SYMMETRY IR-INTENSITY

\begin{tabular}{|c|c|c|c|c|c|}
\hline 1 & vib & 626.81 & 0.00 & $A^{\prime \prime}$ & 67.8742 \\
\hline 2 & vib & 637.99 & 0.00 & $A^{\prime}$ & 1.6352 \\
\hline 3 & vib & 765.62 & 0.00 & $A^{\prime \prime}$ & 1.0416 \\
\hline 4 & vib & 878.39 & 0.00 & $A^{\prime}$ & 79.2254 \\
\hline 5 & vib & 1007.24 & 0.00 & $A^{\prime}$ & 33.6263 \\
\hline 6 & vib & 1040.07 & 0.00 & $A^{\prime}$ & 0.7425 \\
\hline 7 & vib & 1082.33 & 0.00 & $A^{\prime \prime}$ & 1.0762 \\
\hline 8 & vib & 1508.35 & 0.00 & $A^{\prime}$ & 1.5938 \\
\hline 9 & vib & 1769.14 & 0.00 & $A^{\prime}$ & 17.9807 \\
\hline 10 & vib & 3067.17 & 0.00 & $A^{\prime}$ & 42.2857 \\
\hline 11 & vib & 3146.14 & 0.00 & $A^{\prime \prime}$ & 21.1440 \\
\hline 12 & vib & 3333.33 & 0.00 & $A^{\prime}$ & 16.7079 \\
\hline
\end{tabular}




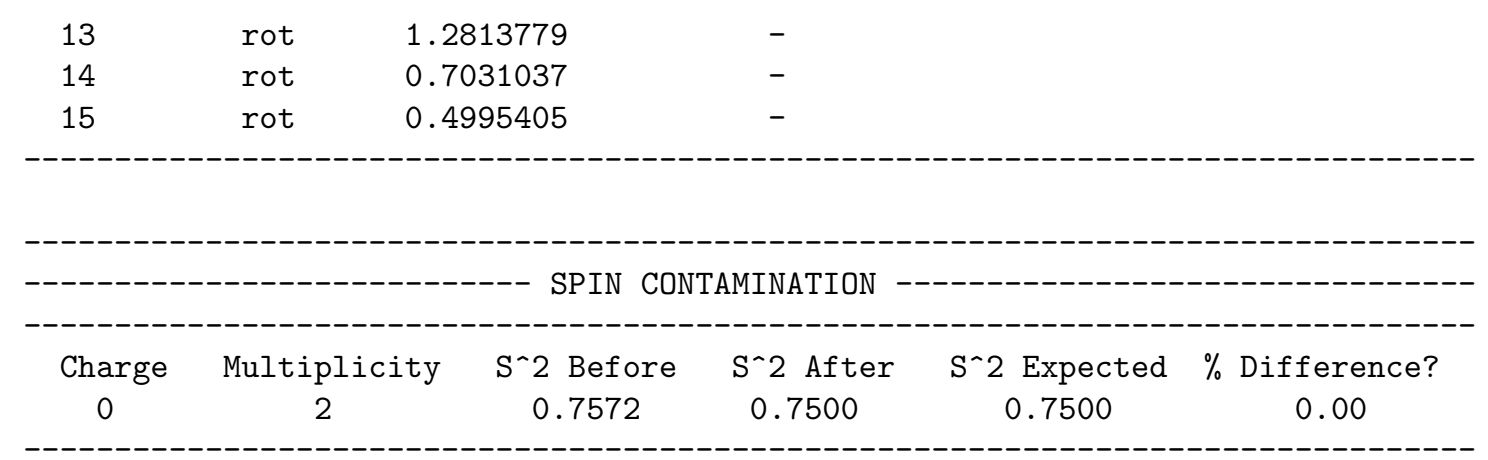

\section{1HydroxyEthylGaucheAnti.log}

$$
\text { Charge }=0 \text { Multiplicity }=2 \text { Stoichiometry } \quad \text { C2H50(2) }
$$

\begin{tabular}{|c|c|c|c|c|c|}
\hline \multirow{2}{*}{$\begin{array}{l}\text { Center } \\
\text { Number }\end{array}$} & \multirow{2}{*}{$\begin{array}{l}\text { Atomic } \\
\text { Number }\end{array}$} & \multirow{2}{*}{$\begin{array}{l}\text { Atomic } \\
\text { Type }\end{array}$} & \multicolumn{3}{|c|}{ Coordinates (Angstroms) } \\
\hline & & & $\mathrm{X}$ & Y & $\mathrm{Z}$ \\
\hline 1 & 6 & 0 & 1.227398 & -0.162950 & 0.011162 \\
\hline 2 & 1 & 0 & 2.028862 & 0.544818 & -0.199780 \\
\hline 3 & 1 & 0 & 1.406081 & -0.582754 & 1.012071 \\
\hline 4 & 6 & 0 & -0.089843 & 0.504011 & -0.096190 \\
\hline 5 & 1 & 0 & -0.232275 & 1.526456 & 0.237508 \\
\hline 6 & 8 & 0 & -1.168947 & -0.339848 & 0.019837 \\
\hline 7 & 1 & 0 & -1.980866 & 0.174487 & 0.000056 \\
\hline 8 & 1 & 0 & 1.304440 & -0.990586 & -0.698381 \\
\hline
\end{tabular}

FREQUENCIES AND ROTATIONAL CONSTANTS

$\begin{array}{cccccr}\text { INDEX NO. DOF TYPE } & \text { CM-1 (UNSCALED) } & \text { CM-1 (SCALED BY ) } & \text { SYMMETRY } & \text { IR-INTENSITY } \\ 1 & \text { vib } & 179.73 & 0.00 & \mathrm{~A} & 3.7505 \\ 2 & \text { vib } & 353.70 & 0.00 & \mathrm{~A} & 121.6161 \\ 3 & \text { vib } & 408.40 & 0.00 & \mathrm{~A} & 9.5352 \\ 4 & \text { vib } & 558.79 & 0.00 & \mathrm{~A} & 17.9636 \\ 5 & \text { vib } & 929.37 & 0.00 & \mathrm{~A} & 9.5440 \\ 6 & \text { vib } & 1022.53 & 0.00 & \mathrm{~A} & 5.9973 \\ 7 & \text { vib } & 1058.51 & 0.00 & \mathrm{~A} & 33.5979 \\ 8 & \text { vib } & 1207.77 & 0.00 & \mathrm{~A} & 42.0327 \\ 9 & \text { vib } & 1274.72 & 0.00 & \mathrm{~A} & 122.3160 \\ 10 & \text { vib } & 1397.06 & 0.00 & \mathrm{~A} & 0.1737 \\ 11 & \text { vib } & 1447.96 & 0.00 & \mathrm{~A} & 15.0065 \\ 12 & \text { vib } & 1465.11 & 0.00 & \mathrm{~A} & 10.7999 \\ 13 & \text { vib } & 1490.42 & 0.00 & \mathrm{~A} & 1.3569 \\ 14 & \text { vib } & 2944.54 & 0.00 & \mathrm{~A} & 49.9017 \\ 15 & \text { vib } & 3037.01 & 0.00 & \mathrm{~A} & 19.8623 \\ 16 & \text { vib } & 3096.14 & 0.00 & \mathrm{~A} & 10.0639\end{array}$




\begin{tabular}{llrlll}
17 & vib & 3128.87 & 0.00 & A & 45.9392 \\
18 & vib & 3831.16 & 0.00 & A & 54.5633 \\
19 & rot & 1.5484878 & - & & \\
20 & rot & 0.3148532 & - & & \\
21 & rot & 0.2764095 & - & & \\
\hline
\end{tabular}

SPIN CONTAMINATION

Charge Multiplicity $\quad S^{\wedge} 2$ Before $S^{\wedge} 2$ After $S^{\wedge} 2$ Expected \% Difference?
0
2
0.7536
0.7500
0.7500
0.00

\section{1HydroxyEthylGaucheSyn.log}

\begin{tabular}{|c|c|c|c|c|c|}
\hline \multirow{2}{*}{$\begin{array}{l}\text { Center } \\
\text { Number }\end{array}$} & \multirow{2}{*}{$\begin{array}{l}\text { Atomic } \\
\text { Number }\end{array}$} & \multirow{2}{*}{$\begin{array}{c}\text { Atomic } \\
\text { Type }\end{array}$} & \multicolumn{3}{|c|}{ Coordinates (Angstroms) } \\
\hline & & & $\mathrm{X}$ & Y & $\mathrm{Z}$ \\
\hline 1 & 6 & 0 & 1.208137 & -0.179314 & 0.019536 \\
\hline 2 & 1 & 0 & 2.032701 & 0.494909 & -0.211402 \\
\hline 3 & 1 & 0 & 1.377333 & -0.574473 & 1.033490 \\
\hline 4 & 6 & 0 & -0.091435 & 0.525404 & -0.112209 \\
\hline 5 & 1 & 0 & -0.206898 & 1.559417 & 0.180168 \\
\hline 6 & 8 & 0 & -1.259682 & -0.177610 & 0.047124 \\
\hline 7 & 1 & 0 & -1.103187 & -1.106394 & -0.154058 \\
\hline 8 & 1 & 0 & 1.277290 & -1.029119 & -0.669151 \\
\hline
\end{tabular}

FREQUENCIES AND ROTATIONAL CONSTANTS

INDEX NO. DOF TYPE CM-1(UNSCALED) CM-1 (SCALED BY) SYMMETRY IR-INTENSITY

\begin{tabular}{|c|c|c|c|c|c|}
\hline 1 & vib & 193.76 & 0.00 & $\mathrm{~A}$ & 3.3319 \\
\hline 2 & vib & 332.40 & 0.00 & $\mathrm{~A}$ & 56.9254 \\
\hline 3 & vib & 407.95 & 0.00 & $\mathrm{~A}$ & 7.9881 \\
\hline 4 & vib & 570.32 & 0.00 & $\mathrm{~A}$ & 64.5138 \\
\hline 5 & vib & 915.28 & 0.00 & $\mathrm{~A}$ & 13.5848 \\
\hline 6 & vib & 1019.30 & 0.00 & $\mathrm{~A}$ & 7.0872 \\
\hline 7 & vib & 1061.41 & 0.00 & $\mathrm{~A}$ & 41.0813 \\
\hline 8 & vib & 1200.20 & 0.00 & $\mathrm{~A}$ & 102.1660 \\
\hline 9 & vib & 1309.21 & 0.00 & A & 1.8212 \\
\hline 10 & vib & 1401.34 & 0.00 & A & 12.8362 \\
\hline 11 & vib & 1434.83 & 0.00 & A & 76.2583 \\
\hline 12 & vib & 1467.44 & 0.00 & A & 6.8414 \\
\hline 13 & vib & 1485.91 & 0.00 & A & 1.0775 \\
\hline 14 & vib & 2927.74 & 0.00 & A & 57.6615 \\
\hline
\end{tabular}




$\begin{array}{llllll}15 & \text { vib } & 3002.23 & 0.00 & \text { A } & 30.8960 \\ 16 & \text { vib } & 3093.42 & 0.00 & \text { A } & 14.0474 \\ 17 & \text { vib } & 3186.27 & 0.00 & \text { A } & 19.8245 \\ 18 & \text { vib } & 3801.25 & 0.00 & \text { A } & 31.8149 \\ 19 & \text { rot } & 1.4863395 & - & & \\ 20 & \text { rot } & 0.3148911 & - & \\ 21 & \text { rot } & 0.2750527 & - & \end{array}$

SPIN CONTAMINATION

$\begin{array}{cccccc}\text { Charge } & \text { Multiplicity } & \mathrm{S}^{\wedge} 2 \text { Before } & \mathrm{S}^{\wedge} 2 \text { After } & \mathrm{S}^{\wedge} 2 \text { Expected } & \% \text { Difference? } \\ 0 & 2 & 0.7536 & 0.7500 & 0.7500 & 0.00\end{array}$

\section{1Propynyl.log}

\section{2-HydroxyEthyl-anti-freqs.log}

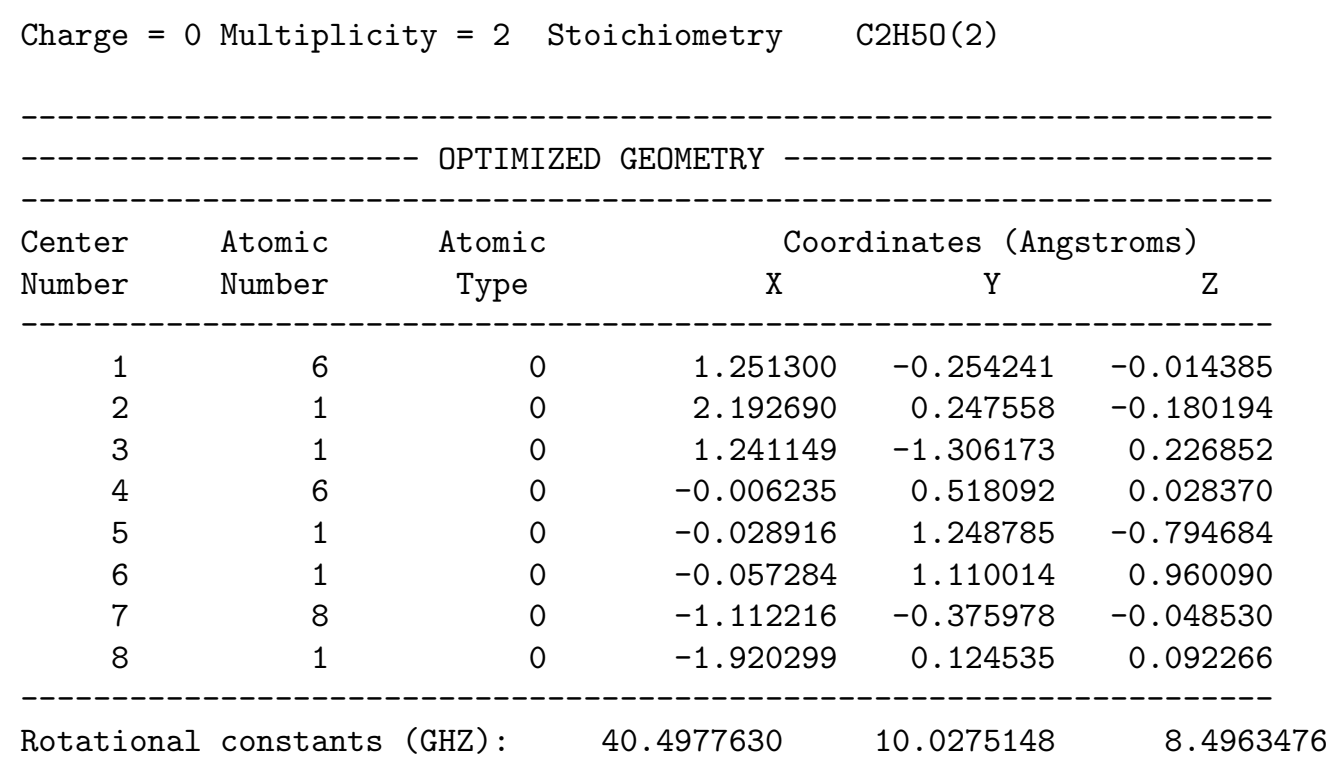

FREQUENCIES AND ROTATIONAL CONSTANTS

INDEX NO. DOF TYPE CM-1(UNSCALED) CM-1(SCALED BY) SYMMETRY IR-INTENSITY

\begin{tabular}{|c|c|c|c|c|c|}
\hline 1 & vib & 110.25 & 0.00 & A & 23.0895 \\
\hline 2 & vib & 265.92 & 0.00 & A & 86.8293 \\
\hline 3 & vib & 412.99 & 0.00 & A & 27.6015 \\
\hline 4 & vib & 458.79 & 0.00 & A & 32.4654 \\
\hline 5 & vib & 867.67 & 0.00 & A & 20.2806 \\
\hline 6 & vib & 957.16 & 0.00 & A & 9.4548 \\
\hline 7 & vib & 1060.03 & 0.00 & A & 29.6259 \\
\hline 8 & vib & 1109.33 & 0.00 & $\mathrm{~A}$ & 48.5462 \\
\hline
\end{tabular}




$\begin{array}{rrrrrr}9 & \text { vib } & 1217.36 & 0.00 & \text { A } & 6.5564 \\ 10 & \text { vib } & 1269.54 & 0.00 & \text { A } & 58.5510 \\ 11 & \text { vib } & 1422.89 & 0.00 & \text { A } & 0.8917 \\ 12 & \text { vib } & 1460.65 & 0.00 & \text { A } & 5.5141 \\ 13 & \text { vib } & 1492.91 & 0.00 & \text { A } & 6.7237 \\ 14 & \text { vib } & 2877.94 & 0.00 & \text { A } & 62.0845 \\ 15 & \text { vib } & 2933.33 & 0.00 & \text { A } & 59.7671 \\ 16 & \text { vib } & 3155.51 & 0.00 & \text { A } & 5.3259 \\ 17 & \text { vib } & 3265.16 & 0.00 & \text { A } & 25.3811 \\ 18 & \text { vib } & 3830.81 & 0.00 & & \\ 19 & \text { rot } & 1.3508600 & - & & \\ 20 & \text { rot } & 0.3344819 & - & & \\ 21 & \text { rot } & 0.2834076 & - & & \end{array}$

SPIN CONTAMINATION

$\begin{array}{cccccc}\text { Charge } & \text { Multiplicity } & \mathrm{S}^{\wedge} 2 \text { Before } & \mathrm{S}^{\wedge} 2 \text { After } & \mathrm{S}^{\wedge} 2 \text { Expected } & \% \text { Difference? } \\ 0 & 2 & 0.7540 & 0.7500 & 0.7500 & 0.00\end{array}$

\section{2-Hydroxyethyl-s-anti.log}

Charge $=0$ Multiplicity $=2$ Stoichiometry $\mathrm{C} 2 \mathrm{H} 50(2)$

\begin{tabular}{|c|c|c|c|c|c|}
\hline \multirow{2}{*}{$\begin{array}{l}\text { Center } \\
\text { Number }\end{array}$} & \multirow{2}{*}{$\begin{array}{l}\text { Atomic } \\
\text { Number }\end{array}$} & \multirow{2}{*}{$\begin{array}{c}\text { Atomic } \\
\text { Type }\end{array}$} & \multicolumn{3}{|c|}{ Coordinates (Angstroms) } \\
\hline & & & $\mathrm{X}$ & Y & Z \\
\hline 1 & 6 & 0 & 1.251306 & -0.254239 & -0.014212 \\
\hline 2 & 1 & 0 & 1.241464 & -1.305785 & 0.228726 \\
\hline 3 & 1 & 0 & 2.192521 & 0.247269 & -0.181813 \\
\hline 4 & 6 & 0 & -0.006220 & 0.518069 & 0.028468 \\
\hline 5 & 1 & 0 & -0.028575 & 1.249154 & -0.794252 \\
\hline 6 & 1 & 0 & -0.057638 & 1.109493 & 0.960468 \\
\hline 7 & 8 & 0 & -1.112275 & -0.375881 & -0.049263 \\
\hline 8 & 1 & 0 & -1.920088 & 0.123930 & 0.095436 \\
\hline
\end{tabular}

FREQUENCIES AND ROTATIONAL CONSTANTS

$\begin{array}{cccccr}\text { INDEX NO. } & \text { DOF TYPE } & \text { CM-1 (UNSCALED) } & \text { CM-1 (SCALED BY }) & \text { SYMMETRY IR-INTENSITY } \\ 1 & \text { vib } & 116.30 & 0.00 & A & 25.8493 \\ 2 & \text { vib } & 258.98 & 0.00 & A & 84.2815 \\ 3 & \text { vib } & 413.71 & 0.00 & \mathrm{~A} & 26.5631 \\ 4 & \text { vib } & 460.28 & 0.00 & \mathrm{~A} & 33.2414 \\ 5 & \text { vib } & 867.61 & 0.00 & \mathrm{~A} & 20.2407 \\ 6 & \text { vib } & 957.13 & 0.00 & \mathrm{~A} & 9.4438\end{array}$




\begin{tabular}{|c|c|c|c|c|c|}
\hline 7 & vib & 1060.26 & 0.00 & $\mathrm{~A}$ & 30.1715 \\
\hline 8 & vib & 1109.39 & 0.00 & $\mathrm{~A}$ & 48.2569 \\
\hline 9 & vib & 1217.34 & 0.00 & $\mathrm{~A}$ & 6.8052 \\
\hline 10 & vib & 1269.44 & 0.00 & $\mathrm{~A}$ & 58.1232 \\
\hline 11 & vib & 1422.94 & 0.00 & $\mathrm{~A}$ & 0.8972 \\
\hline 12 & vib & 1460.74 & 0.00 & $\mathrm{~A}$ & 5.5005 \\
\hline 13 & vib & 1492.81 & 0.00 & $\mathrm{~A}$ & 6.7325 \\
\hline 14 & vib & 2877.43 & 0.00 & $\mathrm{~A}$ & 62.0050 \\
\hline 15 & vib & 2932.89 & 0.00 & $A$ & 59.7904 \\
\hline 16 & vib & 3155.09 & 0.00 & $\mathrm{~A}$ & 5.3260 \\
\hline 17 & vib & 3264.67 & 0.00 & $\mathrm{~A}$ & 5.3956 \\
\hline 18 & vib & 3829.86 & 0.00 & $\mathrm{~A}$ & 25.7899 \\
\hline 19 & rot & 1.3508446 & - & & \\
\hline 20 & rot & 0.3344519 & - & & \\
\hline 21 & rot & 0.2834139 & - & & \\
\hline
\end{tabular}

SPIN CONTAMINATION

$\begin{array}{cccccc}\text { Charge } & \text { Multiplicity } & \mathrm{S}^{\wedge} 2 \text { Before } & \mathrm{S}^{\wedge} 2 \text { After } & \mathrm{S}^{\wedge} 2 \text { Expected } & \% \text { Difference? } \\ 0 & 2 & 0.7540 & 0.7500 & 0.7500 & 0.00\end{array}$
0.7540
0.7500
0.7500
0.00

\section{2-HydroxyEthyls-freqs.log}

Charge $=0$ Multiplicity $=2$ Stoichiometry $\quad$ C2H50(2)

\begin{tabular}{|c|c|c|c|c|c|}
\hline \multirow{2}{*}{$\begin{array}{l}\text { Center } \\
\text { Number }\end{array}$} & \multirow{2}{*}{$\begin{array}{l}\text { Atomic } \\
\text { Number }\end{array}$} & \multirow{2}{*}{$\begin{array}{c}\text { Atomic } \\
\text { Type }\end{array}$} & \multicolumn{3}{|c|}{ Coordinates (Angstroms) } \\
\hline & & & $\mathrm{X}$ & $\mathrm{Y}$ & $\mathrm{Z}$ \\
\hline 1 & 6 & 0 & 1.251300 & -0.254241 & -0.014385 \\
\hline 2 & 1 & 0 & 2.192690 & 0.247558 & -0.180194 \\
\hline 3 & 1 & 0 & 1.241149 & -1.306173 & 0.226852 \\
\hline 4 & 6 & 0 & -0.006235 & 0.518092 & 0.028370 \\
\hline 5 & 1 & 0 & -0.028916 & 1.248785 & -0.794684 \\
\hline 6 & 1 & 0 & -0.057284 & 1.110014 & 0.960090 \\
\hline 7 & 8 & 0 & -1.112216 & -0.375978 & -0.048530 \\
\hline 8 & 1 & 0 & -1.920299 & 0.124535 & 0.092266 \\
\hline
\end{tabular}

FREQUENCIES AND ROTATIONAL CONSTANTS

INDEX NO. DOF TYPE CM-1(UNSCALED) CM-1 (SCALED BY) SYMMETRY IR-INTENSITY

$\begin{array}{llllll}1 & \text { vib } & 110.25 & 0.00 & \mathrm{~A} & 23.0895 \\ 2 & \text { vib } & 265.92 & 0.00 & \mathrm{~A} & 86.8293 \\ 3 & \text { vib } & 412.99 & 0.00 & \mathrm{~A} & 27.6015 \\ 4 & \text { vib } & 458.79 & 0.00 & \mathrm{~A} & 32.4654\end{array}$




\begin{tabular}{|c|c|c|c|c|c|}
\hline 5 & vib & 867.67 & 0.00 & A & 20.2806 \\
\hline 6 & vib & 957.16 & 0.00 & A & 9.4548 \\
\hline 7 & vib & 1060.03 & 0.00 & A & 29.6259 \\
\hline 8 & vib & 1109.33 & 0.00 & A & 48.5462 \\
\hline 9 & vib & 1217.36 & 0.00 & A & 6.5564 \\
\hline 10 & vib & 1269.54 & 0.00 & A & 58.5510 \\
\hline 11 & vib & 1422.89 & 0.00 & A & 0.8917 \\
\hline 12 & vib & 1460.65 & 0.00 & A & 5.5141 \\
\hline 13 & vib & 1492.91 & 0.00 & A & 6.7237 \\
\hline 14 & vib & 2877.94 & 0.00 & A & 62.0845 \\
\hline 15 & vib & 2933.33 & 0.00 & A & 59.7671 \\
\hline 16 & vib & 3155.51 & 0.00 & A & 5.3259 \\
\hline 17 & vib & 3265.16 & 0.00 & A & 5.3811 \\
\hline 18 & vib & 3830.81 & 0.00 & A & 25.8069 \\
\hline 19 & rot & 1.3508600 & - & & \\
\hline 20 & rot & 0.3344819 & - & & \\
\hline 21 & rot & 0.2834076 & - & & \\
\hline
\end{tabular}

SPIN CONTAMINATION

$\begin{array}{cccccc}\text { Charge } & \text { Multiplicity } & S^{\wedge} 2 \text { Before } & S^{\wedge} 2 \text { After } & S^{\wedge} 2 \text { Expected } & \% \text { Difference? } \\ 0 & 2 & 0.7540 & 0.7500 & 0.7500 & 0.00\end{array}$

2) LEVEL OF THEORY: B3LYP/CC-PVTZ+D

Charge $=0$ Multiplicity $=2$ Stoichiometry $\quad$ C2H5O(2)

\begin{tabular}{|c|c|c|c|c|c|}
\hline \multirow{2}{*}{$\begin{array}{l}\text { Center } \\
\text { Number }\end{array}$} & \multirow{2}{*}{$\begin{array}{l}\text { Atomic } \\
\text { Number }\end{array}$} & \multirow{2}{*}{$\begin{array}{c}\text { Atomic } \\
\text { Type }\end{array}$} & \multicolumn{3}{|c|}{ Coordinates (Angstroms) } \\
\hline & & & $\mathrm{X}$ & $\mathrm{Y}$ & $\mathrm{Z}$ \\
\hline 1 & 6 & 0 & -1.233818 & -0.270882 & -0.008394 \\
\hline 2 & 1 & 0 & -2.135077 & 0.108873 & -0.467966 \\
\hline 3 & 1 & 0 & -1.281544 & -1.198273 & 0.545528 \\
\hline 4 & 6 & 0 & 0.006232 & 0.537274 & 0.030504 \\
\hline 5 & 1 & 0 & 0.060367 & 1.130672 & 0.952731 \\
\hline 6 & 1 & 0 & 0.019705 & 1.257258 & -0.798163 \\
\hline 7 & 8 & 0 & 1.193907 & -0.254861 & 0.042684 \\
\hline 8 & 1 & 0 & 1.150811 & -0.857984 & -0.706262 \\
\hline
\end{tabular}

FREQUENCIES AND ROTATIONAL CONSTANTS

INDEX NO. DOF TYPE CM-1(UNSCALED) CM-1 (SCALED BY ) SYMMETRY IR-INTENSITY

$\begin{array}{lllllr}1 & \text { vib } & 188.45 & 0.00 & \text { A } & 3.0763 \\ 2 & \text { vib } & 333.37 & 0.00 & A & 103.1483 \\ 3 & \text { vib } & 424.31 & 0.00 & A & 17.3342 \\ 4 & \text { vib } & 539.20 & 0.00 & \text { A } & 44.5914\end{array}$

Page 16 of 107 


$\begin{array}{rrrrrr}5 & \text { vib } & 828.84 & 0.00 & \text { A } & 0.9278 \\ 6 & \text { vib } & 948.82 & 0.00 & \text { A } & 58.5254 \\ 7 & \text { vib } & 1082.64 & 0.00 & \text { A } & 49.6224 \\ 8 & \text { vib } & 1119.35 & 0.00 & \text { A } & 1.1751 \\ 9 & \text { vib } & 1182.22 & 0.00 & \mathrm{~A} & 26.2967 \\ 10 & \text { vib } & 1362.41 & 0.00 & \mathrm{~A} & 7.9748 \\ 11 & \text { vib } & 1398.52 & 0.00 & \mathrm{~A} & 31.9612 \\ 12 & \text { vib } & 1453.20 & 0.00 & \mathrm{~A} & 5.8955 \\ 13 & \text { vib } & 1485.62 & 0.00 & \mathrm{~A} & 2.2439 \\ 14 & \text { vib } & 2963.77 & 0.00 & \mathrm{~A} & 43.9686 \\ 15 & \text { vib } & 2979.84 & 0.00 & \mathrm{~A} & 38.8723 \\ 16 & \text { vib } & 3139.54 & 0.00 & \mathrm{~A} & 7.5264 \\ 17 & \text { vib } & 3246.16 & 0.00 & \mathrm{~A} & 9.9616 \\ 18 & \text { vib } & 3803.04 & 0.00 & \mathrm{~A} & 19.8039 \\ 19 & \text { rot } & 1.2898037 & - & & \\ 20 & \text { rot } & 0.3294304 & - & & \\ 21 & \text { rot } & 0.2856355 & - & & \\ -------------------------------------------------------------------------\end{array}$

SPIN CONTAMINATION

$\begin{array}{cccccc}\text { Charge } & \text { Multiplicity } & \mathrm{S}^{\wedge} 2 \text { Before } & \mathrm{S}^{\wedge} 2 \text { After } & \mathrm{S}^{\wedge} 2 \text { Expected } & \% \text { Difference? } \\ 0 & 2 & 0.7539 & 0.7500 & 0.7500 & 0.00\end{array}$

\section{2-Propynyl.log}

Charge $=0$ Multiplicity $=2$ Stoichiometry C3H3(2)

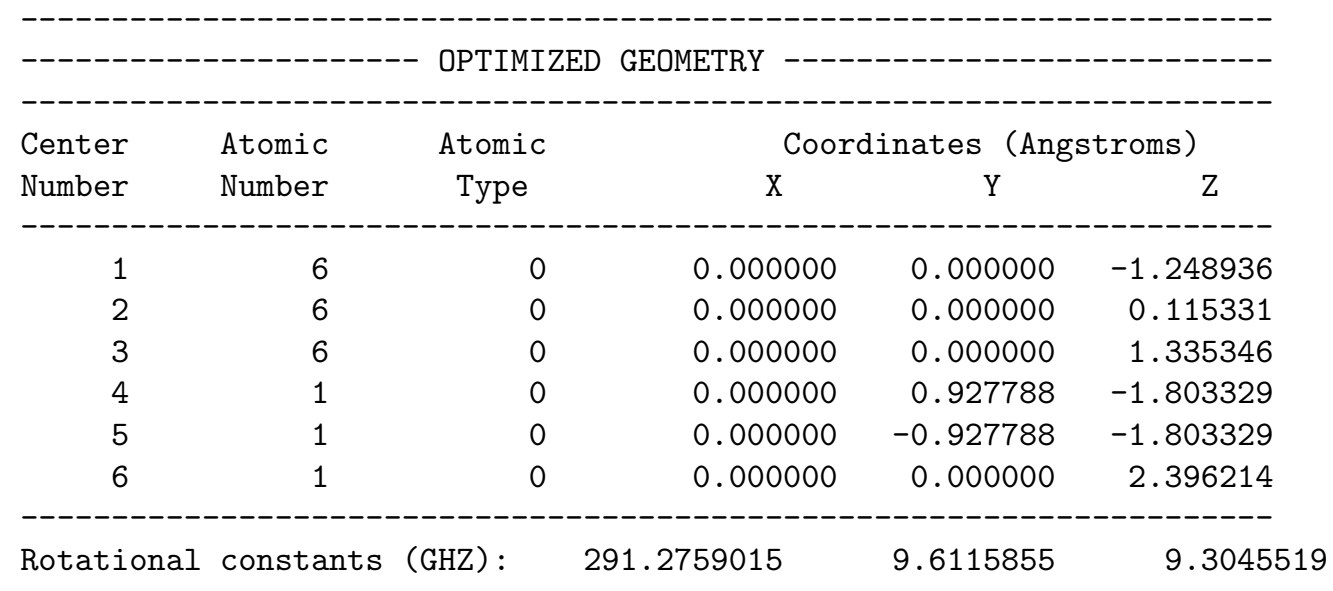

FREQUENCIES AND ROTATIONAL CONSTANTS

INDEX NO. DOF TYPE CM-1(UNSCALED) CM-1(SCALED BY ) SYMMETRY IR-INTENSITY

\begin{tabular}{|c|c|c|c|c|c|}
\hline 1 & vib & 354.90 & 0.00 & B2 & 5.7717 \\
\hline 2 & vib & 407.66 & 0.00 & B1 & 7.6275 \\
\hline 3 & vib & 463.84 & 0.00 & B1 & 49.5903 \\
\hline 4 & vib & 632.89 & 0.00 & B2 & 50.5826 \\
\hline
\end{tabular}




\begin{tabular}{|c|c|c|c|c|c|}
\hline 5 & vib & 691.57 & 0.00 & B1 & 43.3816 \\
\hline 6 & vib & 1033.55 & 0.00 & B2 & 0.9204 \\
\hline 7 & vib & 1090.10 & 0.00 & $\mathrm{~A} 1$ & 1.7335 \\
\hline 8 & vib & 1460.02 & 0.00 & $\mathrm{~A} 1$ & 1.1408 \\
\hline 9 & vib & 2010.84 & 0.00 & $\mathrm{~A} 1$ & 1.2500 \\
\hline 10 & vib & 3142.83 & 0.00 & $\mathrm{~A} 1$ & 2.0416 \\
\hline 11 & vib & 3231.41 & 0.00 & B2 & 0.7813 \\
\hline 12 & vib & 3462.44 & 0.00 & $\mathrm{~A} 1$ & 60.3053 \\
\hline 13 & rot & 9.7159183 & - & & \\
\hline 14 & rot & 0.3206080 & - & & \\
\hline 15 & rot & 0.3103664 & - & & \\
\hline
\end{tabular}

SPIN CONTAMINATION

$\begin{array}{cccccc}\text { Charge } & \text { Multiplicity } & \mathrm{S}^{\wedge} 2 \text { Before } & \mathrm{S}^{\wedge} 2 \text { After } & \mathrm{S}^{\wedge} 2 \text { Expected } & \% \text { Difference? } \\ 0 & 2 & 0.7700 & 0.7502 & 0.7500 & 0.03\end{array}$

\section{23-Butanedione.log}

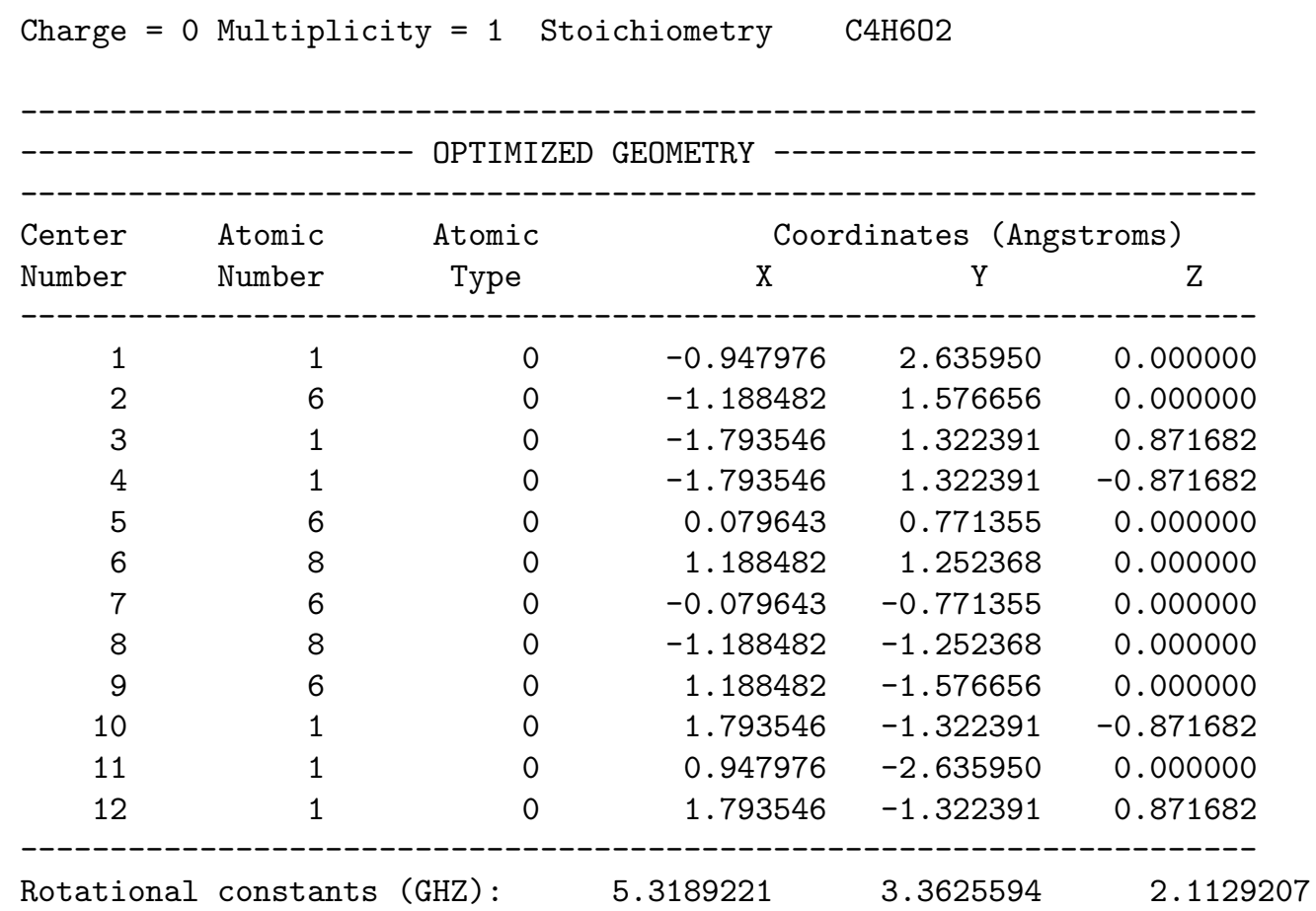

FREQUENCIES AND ROTATIONAL CONSTANTS

INDEX NO. DOF TYPE CM-1(UNSCALED) CM-1(SCALED BY ) SYMMETRY IR-INTENSITY

\begin{tabular}{|c|c|c|c|c|c|}
\hline 1 & vib & 48.72 & 0.00 & $\mathrm{AU}$ & 11.1683 \\
\hline 2 & vib & 115.32 & 0.00 & $B G$ & 0.0000 \\
\hline 3 & vib & 118.63 & 0.00 & $\mathrm{AU}$ & 0.0245 \\
\hline 4 & vib & 240.70 & 0.00 & $\mathrm{BU}$ & 16.3183 \\
\hline
\end{tabular}




$\begin{array}{rrrrrr}5 & \text { vib } & 351.54 & 0.00 & \text { AU } & 5.3358 \\ 6 & \text { vib } & 362.05 & 0.00 & \text { AG } & 0.0000 \\ 7 & \text { vib } & 520.72 & 0.00 & \text { AG } & 0.0000 \\ 8 & \text { vib } & 546.33 & 0.00 & \text { BU } & 41.8339 \\ 9 & \text { vib } & 625.54 & 0.00 & \text { BG } & 0.0000 \\ 10 & \text { vib } & 683.02 & 0.00 & \text { AG } & 0.0000 \\ 11 & \text { vib } & 909.46 & 0.00 & \text { BU } & 28.9554 \\ 12 & \text { vib } & 961.87 & 0.00 & \text { AU } & 5.1043 \\ 13 & \text { vib } & 1014.31 & 0.00 & \text { AG } & 0.0000 \\ 14 & \text { vib } & 1073.40 & 0.00 & \text { BG } & 0.0000 \\ 15 & \text { vib } & 1135.68 & 0.00 & \text { BU } & 80.4836 \\ 16 & \text { vib } & 1276.54 & 0.00 & \text { AG } & 0.0000 \\ 17 & \text { vib } & 1390.35 & 0.00 & \text { BU } & 67.1911 \\ 18 & \text { vib } & 1396.12 & 0.00 & \text { AG } & 0.0000 \\ 19 & \text { vib } & 1457.07 & 0.00 & \text { AG } & 0.0000 \\ 20 & \text { vib } & 1458.13 & 0.00 & \text { BU } & 29.0512 \\ 21 & \text { vib } & 1459.65 & 0.00 & \text { AU } & 19.1142 \\ 22 & \text { vib } & 1464.67 & 0.00 & \text { BG } & 0.0000 \\ 23 & \text { vib } & 1784.38 & 0.00 & \text { AG } & 0.0000 \\ 24 & \text { vib } & 1786.78 & 0.00 & \text { BU } & 247.8465 \\ 25 & \text { vib } & 3043.89 & 0.00 & \text { BU } & 0.3992 \\ 26 & \text { vib } & 3043.96 & 0.00 & \text { AU } & 0.0000 \\ 27 & \text { vib } & 3096.54 & 0.00 & \text { BG } & 0.0979 \\ 28 & \text { vib } & 3096.79 & 0.00 & \text { AG } & 0.0000 \\ 29 & \text { vib } & 3148.95 & 0.00 & & 14.2279 \\ 30 & \text { vib } & 3149.59 & 0.00 & & \\ 31 & \text { rot } & 0.1774201 & - & & \\ 32 & \text { rot } & 0.1121629 & - & - & \\ 33 & \text { rot } & 0.0704794 & & & \\ --------------19 & & \end{array}$

\section{2HydroxyEthylGaucheAnti.log}

Charge $=0$ Multiplicity $=2$ Stoichiometry $\quad$ C2H5O(2)

\begin{tabular}{|c|c|c|c|c|c|}
\hline \multirow{2}{*}{$\begin{array}{l}\text { Center } \\
\text { Number }\end{array}$} & \multirow{2}{*}{$\begin{array}{l}\text { Atomic } \\
\text { Number }\end{array}$} & \multirow{2}{*}{$\begin{array}{c}\text { Atomic } \\
\text { Type }\end{array}$} & \multicolumn{3}{|c|}{ Coordinates (Angstroms) } \\
\hline & & & $\mathrm{X}$ & Y & Z \\
\hline 1 & 6 & 0 & 1.233884 & -0.270805 & -0.008364 \\
\hline 2 & 1 & 0 & 1.282188 & -1.197578 & 0.546494 \\
\hline 3 & 1 & 0 & 2.134567 & 0.108321 & -0.469569 \\
\hline 4 & 6 & 0 & -0.006198 & 0.537231 & 0.030634 \\
\hline 5 & 1 & 0 & -0.019783 & 1.257453 & -0.797841 \\
\hline 6 & 1 & 0 & -0.060557 & 1.130373 & 0.953012 \\
\hline 7 & 8 & 0 & -1.193885 & -0.255013 & 0.042683 \\
\hline 8 & 1 & 0 & -1.151448 & -0.857021 & -0.707176 \\
\hline . & & 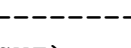 & - & --- & ----- \\
\hline Ro & cons & GHZ) : & 6660056 & 9.8748872 & 8.56333 \\
\hline
\end{tabular}




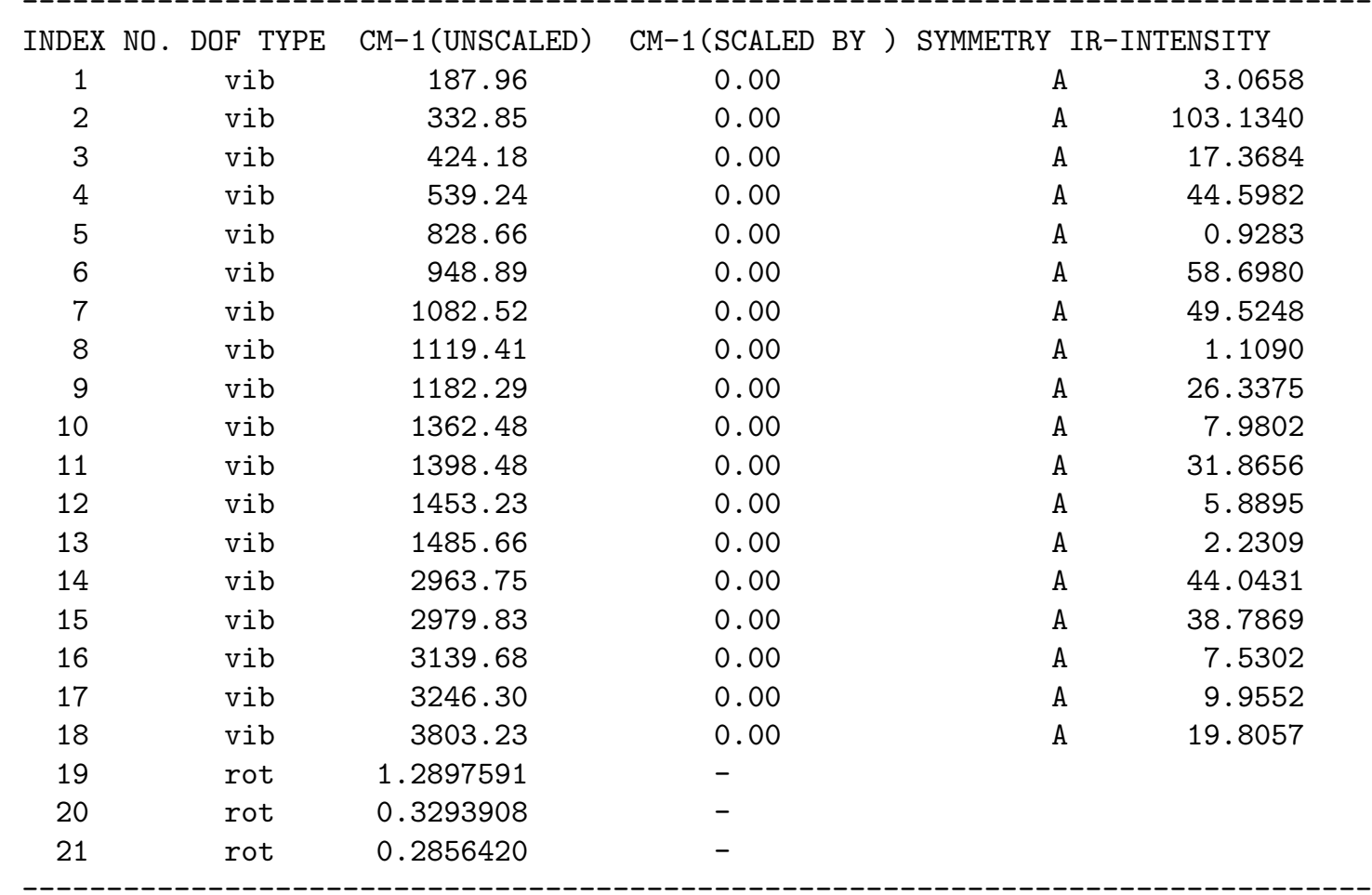

SPIN CONTAMINATION

$\begin{array}{cccccc}\text { Charge } & \text { Multiplicity } & S^{\wedge} 2 \text { Before } & S^{\wedge} 2 \text { After } & S^{\wedge} 2 \text { Expected } & \% \text { Difference? } \\ 0 & 2 & 0.7539 & 0.7500 & 0.7500 & 0.00\end{array}$

\section{2HydroxyEthylGaucheSyn.log}

Charge $=0$ Multiplicity $=2$ Stoichiometry $\quad$ C2H50(2)

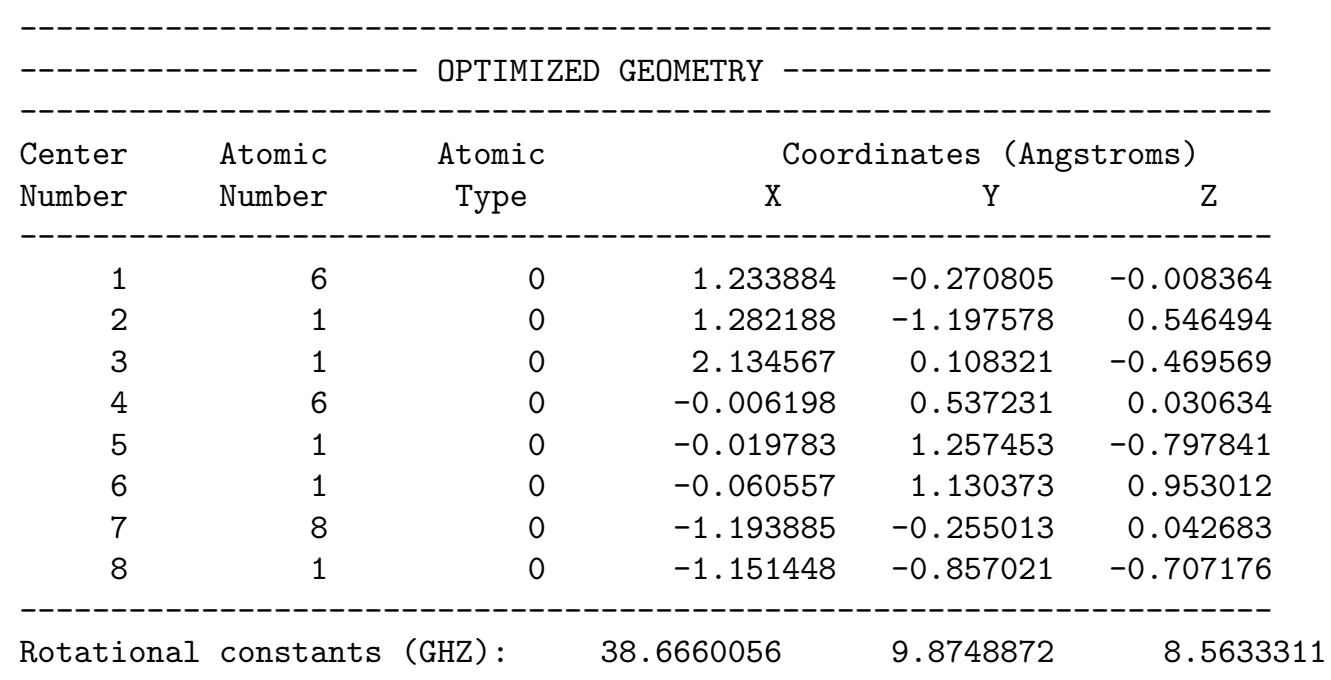


FREQUENCIES AND ROTATIONAL CONSTANTS

\begin{tabular}{|c|c|c|c|c|c|}
\hline INDEX NO. & DOF TYPE & CM-1 (UNSCALED) & CM-1 (SCALED BY ) & SYMMETRY II & TENSITY \\
\hline 1 & vib & 187.96 & 0.00 & A & 3.0658 \\
\hline 2 & vib & 332.85 & 0.00 & A & 103.1340 \\
\hline 3 & vib & 424.18 & 0.00 & A & 17.3684 \\
\hline 4 & vib & 539.24 & 0.00 & A & 44.5982 \\
\hline 5 & vib & 828.66 & 0.00 & A & 0.9283 \\
\hline 6 & vib & 948.89 & 0.00 & A & 58.6980 \\
\hline 7 & vib & 1082.52 & 0.00 & A & 49.5248 \\
\hline 8 & vib & 1119.41 & 0.00 & A & 1.1090 \\
\hline 9 & vib & 1182.29 & 0.00 & A & 26.3375 \\
\hline 10 & vib & 1362.48 & 0.00 & A & 7.9802 \\
\hline 11 & vib & 1398.48 & 0.00 & A & 31.8656 \\
\hline 12 & vib & 1453.23 & 0.00 & A & 5.8895 \\
\hline 13 & vib & 1485.66 & 0.00 & A & 2.2309 \\
\hline 14 & vib & 2963.75 & 0.00 & A & 44.0431 \\
\hline 15 & vib & 2979.83 & 0.00 & A & 38.7869 \\
\hline 16 & vib & 3139.68 & 0.00 & A & 7.5302 \\
\hline 17 & vib & 3246.30 & 0.00 & A & 9.9552 \\
\hline 18 & vib & 3803.23 & 0.00 & A & 19.8057 \\
\hline 19 & rot & 1.2897591 & - & & \\
\hline 20 & rot & 0.3293908 & - & & \\
\hline 21 & rot & 0.2856420 & - & & \\
\hline
\end{tabular}

SPIN CONTAMINATION

$\begin{array}{cccccc}\text { Charge } & \text { Multiplicity } & S^{\wedge} 2 \text { Before } & S^{\wedge} 2 \text { After } & S^{\wedge} 2 \text { Expected } & \% \text { Difference? } \\ 0 & 2 & 0.7539 & 0.7500 & 0.7500 & 0.00\end{array}$

\section{Allyl-freqs.log}

Charge $=0$ Multiplicity $=2$ Stoichiometry C3H5(2)

\begin{tabular}{|c|c|c|c|c|c|}
\hline \multirow{2}{*}{$\begin{array}{l}\text { Center } \\
\text { Number }\end{array}$} & \multirow{2}{*}{$\begin{array}{l}\text { Atomic } \\
\text { Number }\end{array}$} & \multirow{2}{*}{$\begin{array}{c}\text { Atomic } \\
\text { Type }\end{array}$} & \multicolumn{3}{|c|}{ Coordinates (Angstroms) } \\
\hline & & & $\mathrm{X}$ & $\mathrm{Y}$ & $\mathrm{Z}$ \\
\hline 1 & 6 & 0 & 0.000000 & 0.000000 & 0.440593 \\
\hline 2 & 1 & 0 & 0.000000 & 0.000000 & 1.526424 \\
\hline 3 & 6 & 0 & 0.000000 & 1.224045 & -0.195203 \\
\hline 4 & 6 & 0 & 0.000000 & -1.224045 & -0.195203 \\
\hline 5 & 1 & 0 & 0.000000 & 2.149358 & 0.361575 \\
\hline 6 & 1 & 0 & 0.000000 & -2.149358 & 0.361575 \\
\hline 7 & 1 & 0 & 0.000000 & 1.292659 & -1.275349 \\
\hline 8 & 1 & 0 & 0.000000 & -1.292659 & -1.275349 \\
\hline
\end{tabular}




Rotational constants (GHZ): $55.3415560 \quad 10.3904591 \quad 8.7480077$

FREQUENCIES AND ROTATIONAL CONSTANTS

\begin{tabular}{|c|c|c|c|c|c|}
\hline INDEX NO. & DOF TYPE & CM-1 (UNSCALED) & CM-1 (SCALED BY 1) & SYMMETRY & IR-INTENSITY \\
\hline 1 & vib & 429.31 & 429.31 & A1 & 0.0936 \\
\hline 2 & vib & 532.12 & 532.12 & B1 & 15.2727 \\
\hline 3 & vib & 554.23 & 554.23 & A2 & 0.0000 \\
\hline 4 & vib & 797.70 & 797.70 & A2 & 0.0000 \\
\hline 5 & vib & 822.84 & 822.84 & B1 & 72.6785 \\
\hline 6 & vib & 940.15 & 940.15 & B2 & 0.0040 \\
\hline 7 & vib & 1017.72 & 1017.72 & B1 & 19.2276 \\
\hline 8 & vib & 1040.86 & 1040.86 & A1 & 0.0197 \\
\hline 9 & vib & 1210.86 & 1210.86 & B2 & 0.3963 \\
\hline 10 & vib & 1275.61 & 1275.61 & A1 & 1.1224 \\
\hline 11 & vib & 1429.82 & 1429.82 & B2 & 5.6909 \\
\hline 12 & vib & 1516.31 & 1516.31 & B2 & 1.0710 \\
\hline 13 & vib & 1522.64 & 1522.64 & A1 & 2.8703 \\
\hline 14 & vib & 3130.34 & 3130.34 & A1 & 12.4817 \\
\hline 15 & vib & 3137.65 & 3137.65 & B2 & 7.6572 \\
\hline 16 & vib & 3143.66 & 3143.66 & A1 & 5.2082 \\
\hline 17 & vib & 3234.46 & 3234.46 & B2 & 4.9904 \\
\hline 18 & vib & 3237.10 & 3237.10 & A1 & 16.9066 \\
\hline 19 & rot & 1.8459956 & - & & \\
\hline 20 & rot & 0.3465884 & - & & \\
\hline 21 & rot & 0.2918021 & - & & \\
\hline
\end{tabular}

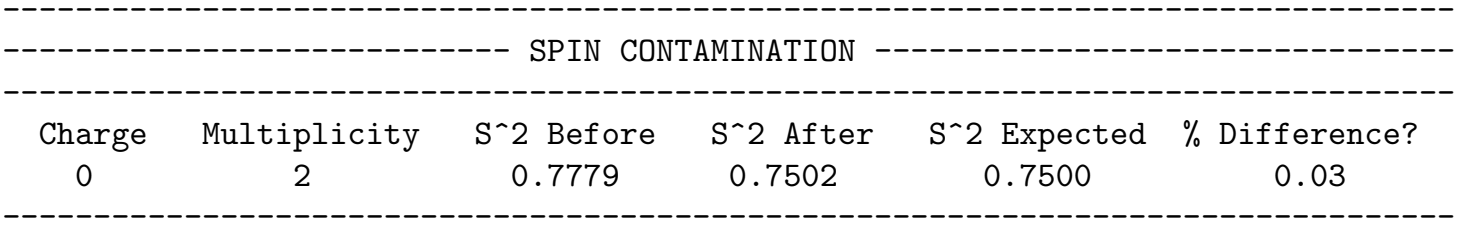

\section{AminoMethyl.log}

Charge = 0 Multiplicity $=2$ Stoichiometry $\quad$ CH4N(2)

\begin{tabular}{|c|c|c|c|c|c|}
\hline \multirow{2}{*}{$\begin{array}{l}\text { Center } \\
\text { Number }\end{array}$} & \multirow{2}{*}{$\begin{array}{l}\text { Atomic } \\
\text { Number }\end{array}$} & \multirow{2}{*}{$\begin{array}{c}\text { Atomic } \\
\text { Type }\end{array}$} & \multicolumn{3}{|c|}{ Coordinates (Angstroms) } \\
\hline & & & $\mathrm{X}$ & $\mathrm{Y}$ & $\mathrm{Z}$ \\
\hline 1 & 6 & 0 & 0.013224 & 0.731374 & 0.000000 \\
\hline 2 & 1 & 0 & 0.273586 & 1.216811 & 0.928506 \\
\hline 3 & 1 & 0 & 0.273586 & 1.216811 & -0.928506 \\
\hline 4 & 7 & 0 & 0.013224 & -0.661992 & 0.000000 \\
\hline 5 & 1 & 0 & -0.359542 & -1.093962 & -0.831747 \\
\hline 6 & 1 & 0 & -0.359542 & -1.093962 & 0.831747 \\
\hline
\end{tabular}




Rotational constants (GHZ): $147.4840519 \quad 27.5183128 \quad 23.8354108$

FREQUENCIES AND ROTATIONAL CONSTANTS

$\begin{array}{ccccrr}\text { INDEX NO. DOF TYPE } & \text { CM-1 (UNSCALED) } & \text { CM-1 (SCALED BY }) & \text { SYMMETRY } & \text { IR-INTENSITY } \\ 1 & \text { vib } & 441.38 & 0.00 & A^{\prime \prime} & 30.8660 \\ 2 & \text { vib } & 597.60 & 0.00 & A^{\prime} & 148.9187 \\ 3 & \text { vib } & 694.01 & 0.00 & A^{\prime} & 136.4624 \\ 4 & \text { vib } & 938.71 & 0.00 & A^{\prime \prime} & 0.0227 \\ 5 & \text { vib } & 1220.08 & 0.00 & A^{\prime} & 22.5208 \\ 6 & \text { vib } & 1324.74 & 0.00 & A^{\prime \prime} & 2.5552 \\ 7 & \text { vib } & 1479.79 & 0.00 & A^{\prime} & 2.1149 \\ 8 & \text { vib } & 1650.59 & 0.00 & A^{\prime} & 29.7342 \\ 9 & \text { vib } & 3141.45 & 0.00 & A^{\prime} & 15.3970 \\ 10 & \text { vib } & 3249.83 & 0.00 & A^{\prime} & 16.6067 \\ 11 & \text { vib } & 3532.16 & 0.00 & A^{\prime \prime} & 10.0997 \\ 12 & \text { vib } & 3631.87 & 0.00 & & \\ 13 & \text { rot } & 4.9195384 & - & & \\ 14 & \text { rot } & 0.9179121 & - & - & \end{array}$

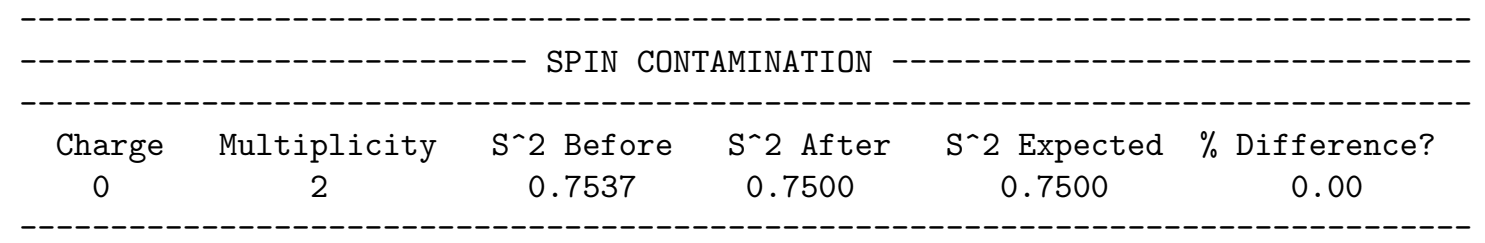

\section{Aminoxyl-H2NO-freqs.log}

Charge $=0$ Multiplicity $=2$ Stoichiometry H2NO(2)

\begin{tabular}{|c|c|c|c|c|c|}
\hline \multirow{2}{*}{$\begin{array}{l}\text { Center } \\
\text { Number }\end{array}$} & \multirow{2}{*}{$\begin{array}{l}\text { Atomic } \\
\text { Number }\end{array}$} & \multirow{2}{*}{$\begin{array}{c}\text { Atomic } \\
\text { Type }\end{array}$} & \multicolumn{3}{|c|}{ Coordinates (Angstroms) } \\
\hline & & & $\mathrm{X}$ & $\mathrm{Y}$ & $\mathrm{Z}$ \\
\hline 1 & 7 & 0 & 0.018154 & 0.541596 & -0.000000 \\
\hline 2 & 1 & 0 & -0.136153 & 1.037772 & 0.872102 \\
\hline 3 & 1 & 0 & -0.136153 & 1.037772 & -0.872102 \\
\hline 4 & 8 & 0 & 0.018154 & -0.733339 & 0.000000 \\
\hline
\end{tabular}

FREQUENCIES AND ROTATIONAL CONSTANTS

INDEX NO. DOF TYPE CM-1(UNSCALED) CM-1(SCALED BY) SYMMETRY IR-INTENSITY
1
vib
352.02
0.00
A'
170.3050 


\begin{tabular}{rrrlrr}
2 & vib & 1270.83 & 0.00 & $A^{\prime \prime}$ & 1.0164 \\
3 & vib & 1372.82 & 0.00 & $A^{\prime}$ & 6.9487 \\
4 & vib & 1660.81 & 0.00 & $A^{\prime}$ & 12.3750 \\
5 & vib & 3403.46 & 0.00 & $A^{\prime}$ & 0.1651 \\
6 & vib & 3531.06 & 0.00 & $A^{\prime \prime}$ & 2.1303 \\
7 & rot & 10.7372430 & - & & \\
8 & rot & 1.1395446 & - & & \\
9 & rot & 1.0348870 & - & \\
\hline
\end{tabular}

\section{AmmoniaOxide.log}

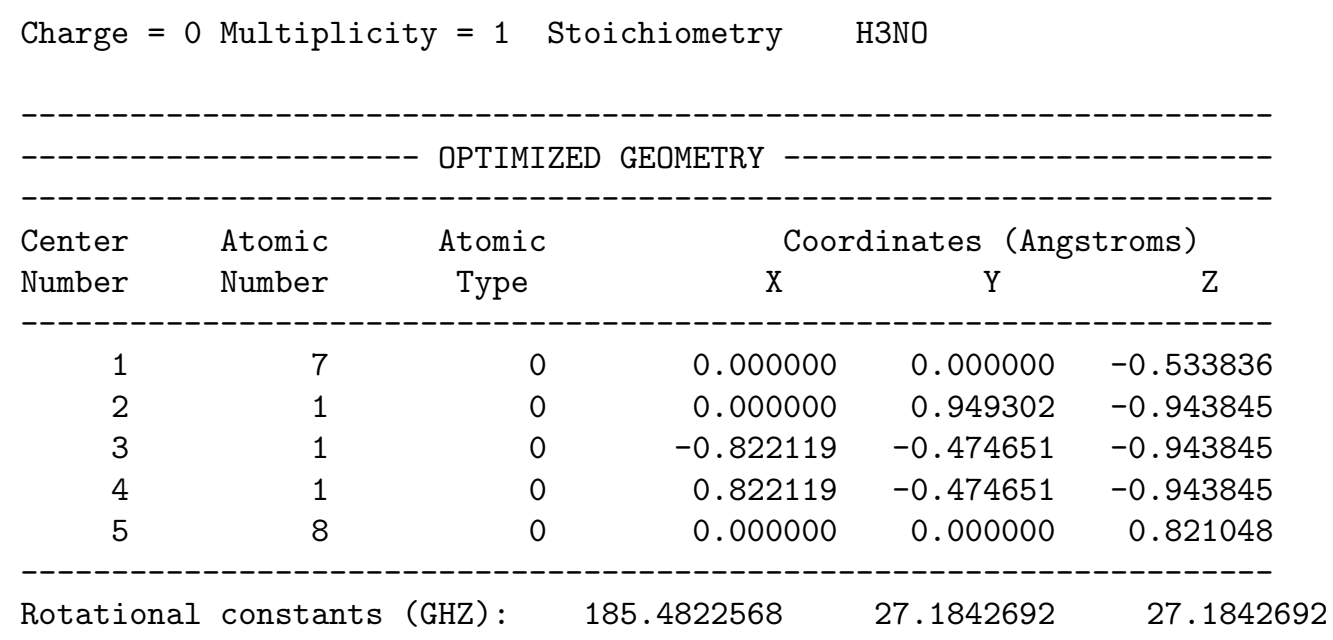

FREQUENCIES AND ROTATIONAL CONSTANTS

$\begin{array}{ccccrr}\text { INDEX NO. } & \text { DOF TYPE } & \text { CM-1 (UNSCALED) } & \text { CM-1 (SCALED BY }) & \text { SYMMETRY } & \text { IR-INTENSITY } \\ 1 & \text { vib } & 944.40 & 0.00 & \mathrm{~A} 1 & 73.7137 \\ 2 & \text { vib } & 1157.63 & 0.00 & \mathrm{E} & 22.4060 \\ 3 & \text { vib } & 1157.64 & 0.00 & \mathrm{E} & 22.4058 \\ 4 & \text { vib } & 1547.33 & 0.00 & \mathrm{~A} 1 & 1.5006 \\ 5 & \text { vib } & 1650.12 & 0.00 & \mathrm{E} & 9.4202 \\ 6 & \text { vib } & 1650.12 & 0.00 & \mathrm{E} & 9.4206 \\ 7 & \text { vib } & 3175.82 & 0.00 & \mathrm{E} & 7.8000 \\ 8 & \text { vib } & 3175.82 & 0.00 & \mathrm{E} & 7.7972 \\ 9 & \text { vib } & 3182.94 & 0.00 & \mathrm{~A} 1 & 116.4206 \\ 10 & \text { rot } & 6.1870221 & - & & \\ 11 & \text { rot } & 0.9067696 & - & & \\ 12 & \text { rot } & 0.9067696 & - & & \\ ----------------------------------------------------------------\end{array}$




\section{Benzene-freqs.log}

\begin{tabular}{|c|c|c|c|c|c|}
\hline \multirow{2}{*}{$\begin{array}{l}\text { Center } \\
\text { Number }\end{array}$} & \multirow{2}{*}{$\begin{array}{l}\text { Atomic } \\
\text { Number }\end{array}$} & \multirow{2}{*}{$\begin{array}{c}\text { Atomic } \\
\text { Type }\end{array}$} & \multicolumn{3}{|c|}{ Coordinates (Angstroms) } \\
\hline & & & $\mathrm{X}$ & Y & Z \\
\hline 1 & 6 & 0 & 0.000000 & 1.390740 & 0.000000 \\
\hline 2 & 6 & 0 & 1.204416 & 0.695370 & 0.000000 \\
\hline 3 & 6 & 0 & -1.204416 & 0.695370 & 0.000000 \\
\hline 4 & 1 & 0 & 2.141508 & 1.236400 & 0.000000 \\
\hline 5 & 1 & 0 & -2.141508 & 1.236400 & 0.000000 \\
\hline 6 & 6 & 0 & 1.204416 & -0.695370 & 0.000000 \\
\hline 7 & 6 & 0 & -1.204416 & -0.695370 & 0.000000 \\
\hline 8 & 1 & 0 & 2.141508 & -1.236400 & 0.000000 \\
\hline 9 & 1 & 0 & -2.141508 & -1.236400 & 0.000000 \\
\hline 10 & 6 & 0 & 0.000000 & -1.390740 & 0.000000 \\
\hline 11 & 1 & 0 & 0.000000 & -2.472800 & 0.000000 \\
\hline 12 & 1 & 0 & 0.000000 & 2.472800 & 0.000000 \\
\hline
\end{tabular}

FREQUENCIES AND ROTATIONAL CONSTANTS

INDEX NO. DOF TYPE CM-1 (UNSCALED) CM-1 (SCALED BY ) SYMMETRY IR-INTENSITY

$\begin{array}{rrrrrr}1 & \text { vib } & 415.18 & 0.00 & \text { E2U } & 0.0000 \\ 2 & \text { vib } & 415.19 & 0.00 & \text { E2U } & 0.0000 \\ 3 & \text { vib } & 624.08 & 0.00 & \text { E2G } & 0.0000 \\ 4 & \text { vib } & 624.08 & 0.00 & \text { E2G } & 0.0000 \\ 5 & \text { vib } & 694.45 & 0.00 & \text { A2U } & 103.7876 \\ 6 & \text { vib } & 727.12 & 0.00 & \text { B2G } & 0.0000 \\ 7 & \text { vib } & 870.23 & 0.00 & \text { E1G } & 0.0000 \\ 8 & \text { vib } & 870.23 & 0.00 & \text { E1G } & 0.0000 \\ 9 & \text { vib } & 990.81 & 0.00 & \text { E2U } & 0.0000 \\ 10 & \text { vib } & 990.81 & 0.00 & \text { E2U } & 0.0000 \\ 11 & \text { vib } & 1015.35 & 0.00 & \text { A1G } & 0.0000 \\ 12 & \text { vib } & 1024.14 & 0.00 & \text { B2G } & 0.0000 \\ 13 & \text { vib } & 1030.58 & 0.00 & \text { B1U } & 0.0000 \\ 14 & \text { vib } & 1062.02 & 0.00 & \text { E1U } & 4.7354 \\ 15 & \text { vib } & 1062.03 & 0.00 & \text { E1U } & 4.7337 \\ 16 & \text { vib } & 1176.53 & 0.00 & \text { B2U } & 0.0000 \\ 17 & \text { vib } & 1200.29 & 0.00 & \text { E2G } & 0.0000 \\ 18 & \text { vib } & 1200.29 & 0.00 & \text { E2G } & 0.0000 \\ 19 & \text { vib } & 1335.05 & 0.00 & \text { B2U } & 0.0000 \\ 20 & \text { vib } & 1389.38 & 0.00 & \text { A2G } & 0.0000 \\ 21 & \text { vib } & 1518.60 & 0.00 & \text { E1U } & 7.9483 \\ 22 & \text { vib } & 1518.60 & 0.00 & \text { E1U } & 7.9525 \\ 23 & \text { vib } & 1637.40 & 0.00 & \text { E2G } & 0.0000 \\ 24 & \text { vib } & 1637.40 & 0.00 & \text { E2G } & 0.0000\end{array}$




\begin{tabular}{llrllr}
25 & vib & 3157.63 & 0.00 & B1U & 0.0000 \\
26 & vib & 3167.44 & 0.00 & E2G & 0.0000 \\
27 & vib & 3167.44 & 0.00 & E2G & 0.0000 \\
28 & vib & 3183.18 & 0.00 & E1U & 39.3743 \\
29 & vib & 3183.18 & 0.00 & E1U & 39.3753 \\
30 & vib & 3193.28 & 0.00 & A1G & 0.0000 \\
31 & rot & 0.1913087 & - & & \\
32 & rot & 0.1913087 & - & & \\
33 & rot & 0.0956543 & - & & \\
\hline
\end{tabular}

\section{CCNN-freqs.log}

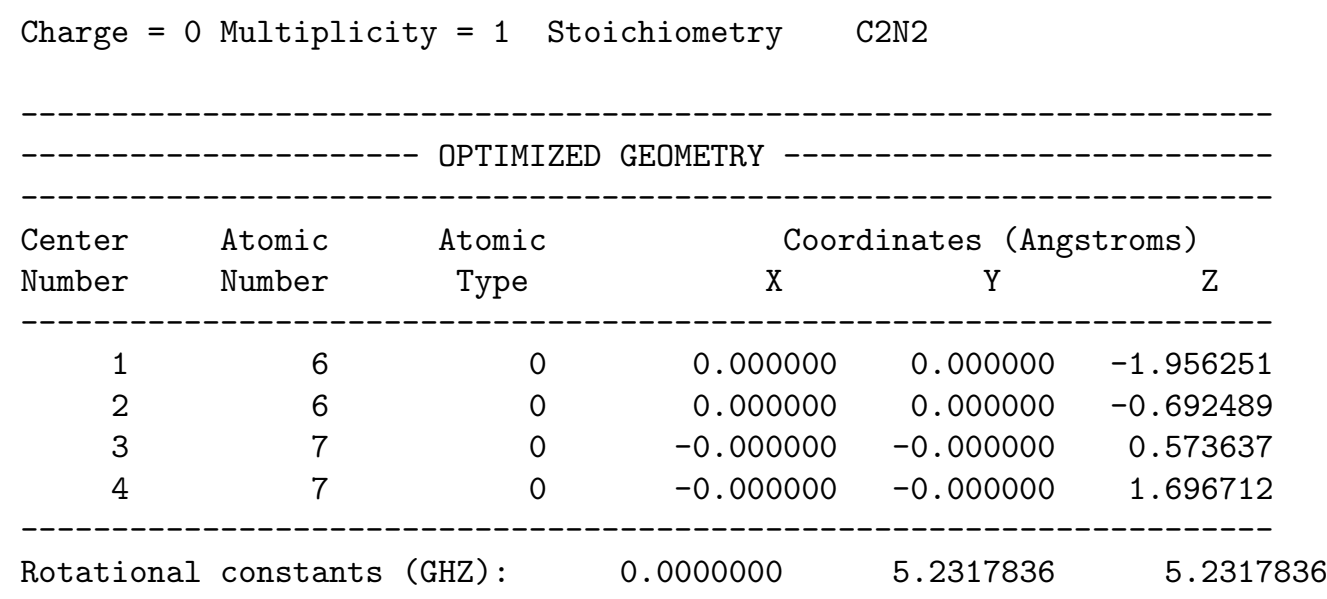

FREQUENCIES AND ROTATIONAL CONSTANTS

\begin{tabular}{|c|c|c|c|c|c|}
\hline INDEX NO. & DOF TYPE & CM-1 (UNSCALED) & CM-1 (SCALED BY ) & SYMMETRY I & TENSITY \\
\hline 1 & vib & 165.20 & 0.00 & PI & 5.1175 \\
\hline 2 & vib & 165.20 & 0.00 & PI & 5.1175 \\
\hline 3 & vib & 564.37 & 0.00 & PI & 9.2252 \\
\hline 4 & vib & 564.37 & 0.00 & PI & 9.2252 \\
\hline 5 & vib & 987.38 & 0.00 & $\mathrm{SG}$ & 0.1852 \\
\hline 6 & vib & 2024.32 & 0.00 & $\mathrm{SG}$ & 247.3227 \\
\hline 7 & vib & 2291.89 & 0.00 & $\mathrm{SG}$ & 800.0915 \\
\hline 8 & vib & 0.00 & 0.00 & & \\
\hline 9 & vib & 0.00 & 0.00 & & \\
\hline 10 & rot & 0.0000000 & - & & \\
\hline 11 & rot & 0.1745135 & - & & \\
\hline 12 & rot & 0.1745135 & - & & \\
\hline
\end{tabular}

\section{2 ch2chchch3-cis.log}

Charge $=0$ Multiplicity $=2$ Stoichiometry $\quad$ C4H7(2) 


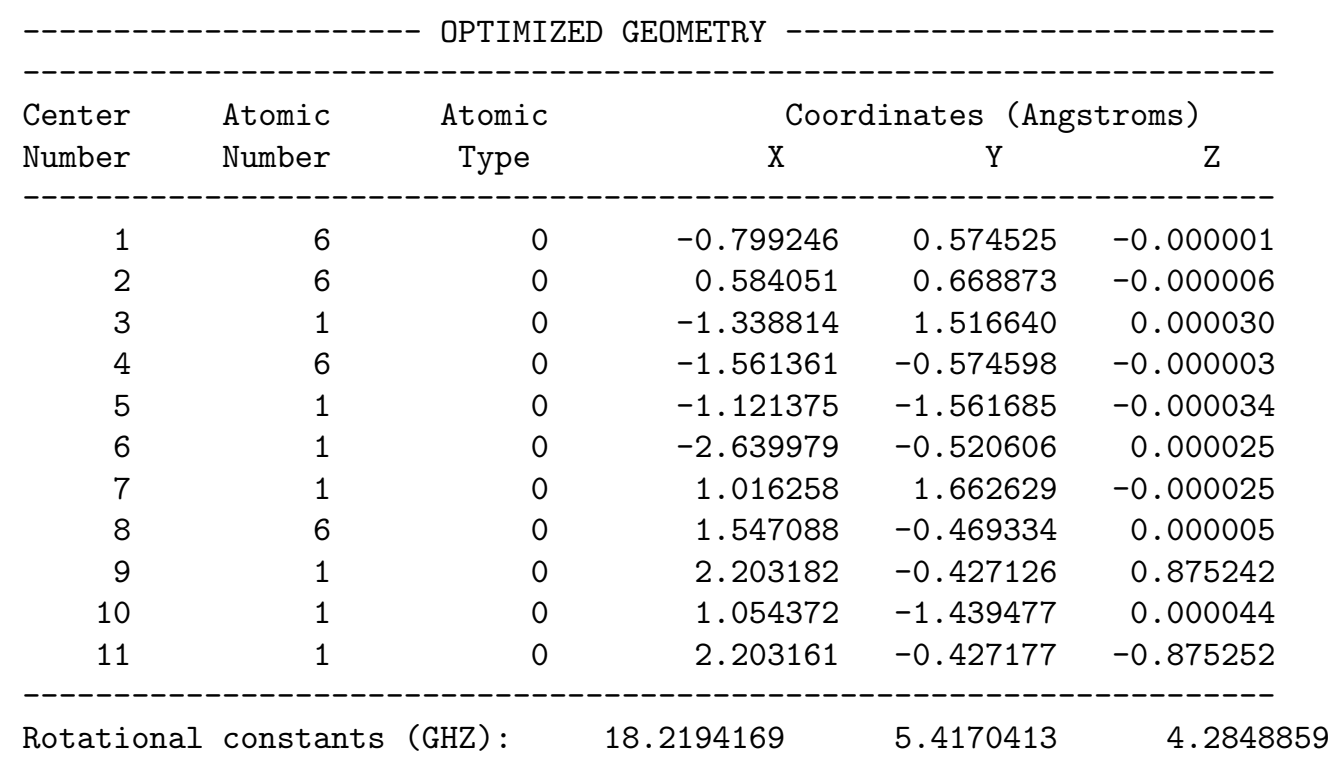

FREQUENCIES AND ROTATIONAL CONSTANTS

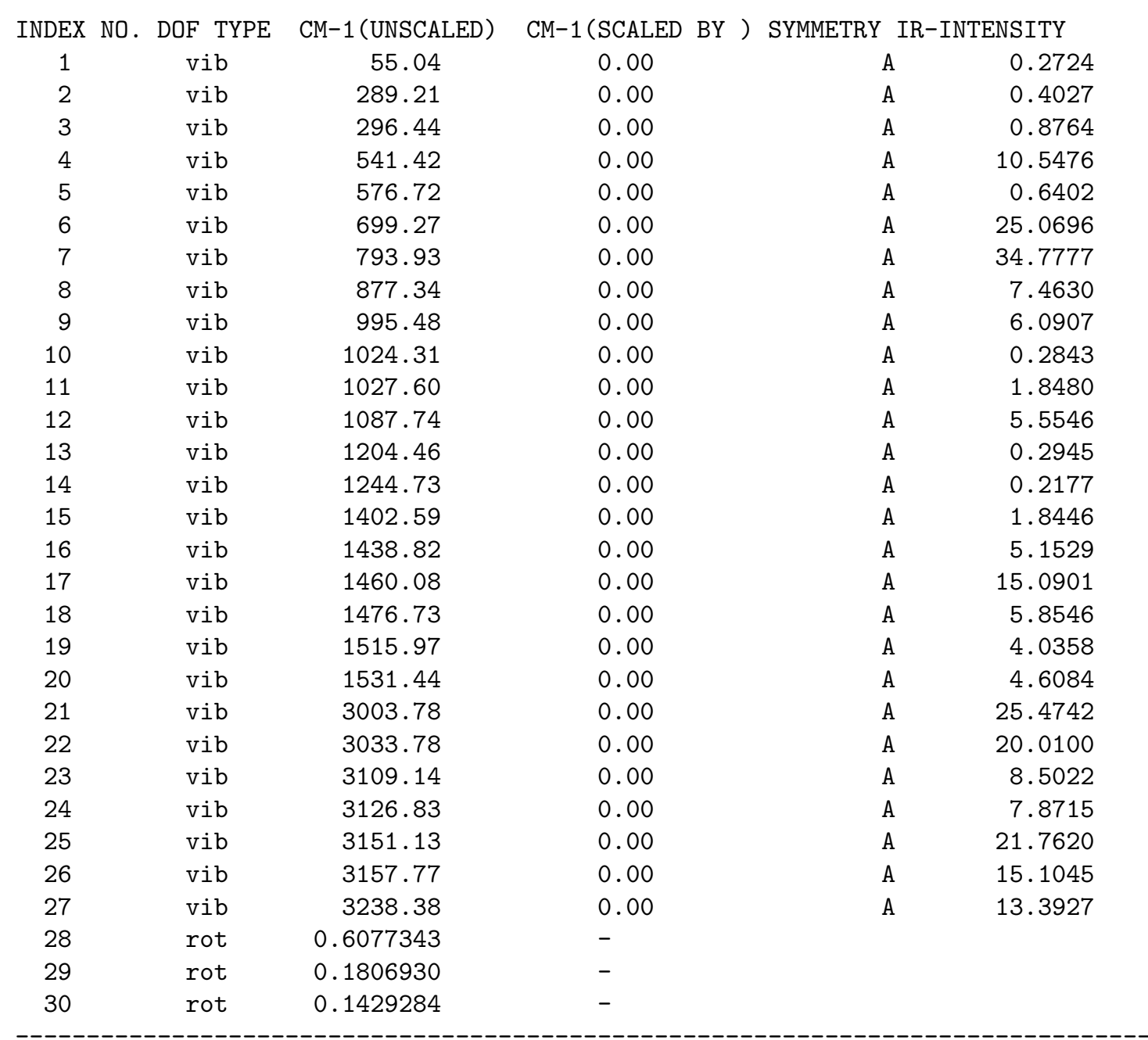




$\begin{array}{cccccc}\text { Charge } & \text { Multiplicity } & S^{\wedge} 2 \text { Before } & S^{\wedge} 2 \text { After } & S^{\wedge} 2 \text { Expected } & \% \text { Difference? } \\ 0 & 2 & 0.7765 & 0.7502 & 0.7500 & 0.03\end{array}$

\section{HINDERED ROTOR CORRECTIONS}

E (Thermal)

CV $\mathrm{KCal} / \mathrm{Mol}$

$\mathrm{Cal} / \mathrm{Mol}-\mathrm{Kelvin}$

$\mathrm{S}$

VIBRATIONS ONLY: 62.472

HR CORRECTED: $\quad 62.290$

17.830

Cal/Mol-Kelvin

CORRECTED-VIB :

$-0.182$

16.943

73.216

72.565

$-0.887$

$-0.651$

\section{3 ch2chchch3-trans.log}

Charge $=0$ Multiplicity $=2$ Stoichiometry $\quad$ C4H7(2)

\begin{tabular}{|c|c|c|c|c|c|}
\hline \multirow{2}{*}{$\begin{array}{l}\text { Center } \\
\text { Number }\end{array}$} & \multirow{2}{*}{$\begin{array}{l}\text { Atomic } \\
\text { Number }\end{array}$} & \multirow{2}{*}{$\begin{array}{l}\text { Atomic } \\
\text { Type }\end{array}$} & \multicolumn{3}{|c|}{ Coordinates (Angstroms) } \\
\hline & & & $\mathrm{X}$ & $\mathrm{Y}$ & $\mathrm{Z}$ \\
\hline 1 & 6 & 0 & 0.657533 & 0.378620 & 0.000349 \\
\hline 2 & 6 & 0 & -0.473904 & -0.415841 & 0.000331 \\
\hline 3 & 1 & 0 & 0.505838 & 1.455256 & 0.000357 \\
\hline 4 & 1 & 0 & -0.343218 & -1.493652 & 0.000781 \\
\hline 5 & 6 & 0 & 1.957882 & -0.081175 & -0.000289 \\
\hline 6 & 1 & 0 & 2.177181 & -1.140906 & -0.000602 \\
\hline 7 & 1 & 0 & 2.795557 & 0.600280 & -0.000148 \\
\hline 8 & 6 & 0 & -1.871399 & 0.100220 & -0.000252 \\
\hline 9 & 1 & 0 & -2.428550 & -0.252096 & -0.874777 \\
\hline 10 & 1 & 0 & -2.428872 & -0.250053 & 0.874946 \\
\hline 11 & 1 & 0 & -1.898602 & 1.190222 & -0.001386 \\
\hline
\end{tabular}

FREQUENCIES AND ROTATIONAL CONSTANTS

INDEX NO. DOF TYPE CM-1(UNSCALED) CM-1(SCALED BY) SYMMETRY IR-INTENSITY

\begin{tabular}{|c|c|c|c|c|c|}
\hline 1 & vib & 151.25 & 0.00 & A & 0.7789 \\
\hline 2 & vib & 218.20 & 0.00 & A & 1.5180 \\
\hline 3 & vib & 287.41 & 0.00 & A & 0.1962 \\
\hline 4 & vib & 507.03 & 0.00 & A & 0.6999 \\
\hline 5 & vib & 546.31 & 0.00 & A & 5.5740 \\
\hline 6 & vib & 744.82 & 0.00 & A & 0.0728 \\
\hline 7 & vib & 798.92 & 0.00 & A & 49.0297 \\
\hline 8 & vib & 882.68 & 0.00 & A & 6.3764 \\
\hline 9 & vib & 996.71 & 0.00 & $A$ & 4.4217 \\
\hline
\end{tabular}




\begin{tabular}{|c|c|c|c|c|c|}
\hline 10 & vib & 997.20 & 0.00 & $\mathrm{~A}$ & 22.4219 \\
\hline 11 & vib & 1030.84 & 0.00 & A & 2.6454 \\
\hline 12 & vib & 1140.78 & 0.00 & $\mathrm{~A}$ & 0.7422 \\
\hline 13 & vib & 1208.99 & 0.00 & A & 0.7920 \\
\hline 14 & vib & 1287.90 & 0.00 & $\mathrm{~A}$ & 2.0863 \\
\hline 15 & vib & 1348.81 & 0.00 & A & 1.1770 \\
\hline 16 & vib & 1411.97 & 0.00 & A & 1. 2824 \\
\hline 17 & vib & 1473.32 & 0.00 & $\mathrm{~A}$ & 6.0113 \\
\hline 18 & vib & 1477.20 & 0.00 & $\mathrm{~A}$ & 15.6068 \\
\hline 19 & vib & 1512.83 & 0.00 & $\mathrm{~A}$ & 7.0564 \\
\hline 20 & vib & 1528.80 & 0.00 & $\mathrm{~A}$ & 1.4542 \\
\hline 21 & vib & 2994.94 & 0.00 & $\mathrm{~A}$ & 33.1897 \\
\hline 22 & vib & 3024.49 & 0.00 & $\mathrm{~A}$ & 21.7662 \\
\hline 23 & vib & 3086.45 & 0.00 & $\mathrm{~A}$ & 14.4800 \\
\hline 24 & vib & 3113.38 & 0.00 & $\mathrm{~A}$ & 7.2963 \\
\hline 25 & vib & 3133.72 & 0.00 & $\mathrm{~A}$ & 25.3162 \\
\hline 26 & vib & 3141.62 & 0.00 & $\mathrm{~A}$ & 15.9276 \\
\hline 27 & vib & 3235.08 & 0.00 & $\mathrm{~A}$ & 12.1119 \\
\hline 28 & rot & 1.2822507 & - & & \\
\hline 29 & rot & 0.1338332 & - & & \\
\hline 30 & rot & 0.1239337 & - & & \\
\hline
\end{tabular}

\begin{tabular}{|c|c|c|c|c|c|}
\hline Charge & Multiplicity & $\mathrm{S}^{\wedge} 2$ Before & $S^{\wedge} 2$ After & $S^{\wedge} 2$ Expected & $\%$ Difference? \\
\hline 0 & 2 & 0.7767 & 0.7502 & 0.7500 & 0.03 \\
\hline
\end{tabular}

\begin{tabular}{|c|c|c|c|}
\hline & E (Thermal) & CV & $\mathrm{S}$ \\
\hline & $\mathrm{KCal} / \mathrm{Mol}$ & Cal/Mol-Kelvin & Cal/Mol-Kelvin \\
\hline VIBRATIONS ONLY: & 62.327 & 17.915 & 71.567 \\
\hline HR CORRECTED: & 62.360 & 17.714 & 72.004 \\
\hline CORRECTED-VIB: & 0.033 & -0.201 & 0.437 \\
\hline
\end{tabular}

\section{4 ch2chchch3.log}

Charge $=0$ Multiplicity $=2$ Stoichiometry $\quad \mathrm{C} 4 \mathrm{H} 7(2)$

\begin{tabular}{|c|c|c|c|c|c|}
\hline \multirow{2}{*}{$\begin{array}{l}\text { Center } \\
\text { Number }\end{array}$} & \multirow{2}{*}{$\begin{array}{l}\text { Atomic } \\
\text { Number }\end{array}$} & \multirow{2}{*}{$\begin{array}{c}\text { Atomic } \\
\text { Type }\end{array}$} & \multicolumn{3}{|c|}{ Coordinates (Angstroms) } \\
\hline & & & $\mathrm{X}$ & $\mathrm{Y}$ & $\mathrm{Z}$ \\
\hline 1 & 6 & 0 & 1.957710 & 0.081139 & 0.000039 \\
\hline 2 & 6 & 0 & 0.657564 & -0.378626 & -0.000122 \\
\hline 3 & 1 & 0 & 2.177047 & 1.140893 & -0.000142 \\
\hline
\end{tabular}




\begin{tabular}{|c|c|c|c|c|c|}
\hline 4 & 1 & 0 & 2.795708 & -0.599904 & 0.000641 \\
\hline 5 & 1 & 0 & 0.505988 & -1.455273 & -0.000441 \\
\hline 6 & 6 & 0 & -0.473886 & 0.415827 & -0.000003 \\
\hline 7 & 1 & 0 & -0.343152 & 1.493589 & -0.000198 \\
\hline 8 & 6 & 0 & -1.871347 & -0.100225 & 0.000073 \\
\hline 9 & 1 & 0 & -2.428752 & 0.251389 & -0.874620 \\
\hline 10 & 1 & 0 & -1.898561 & -1.190205 & -0.000275 \\
\hline 11 & 1 & 0 & -2.428520 & 0.250820 & 0.875118 \\
\hline
\end{tabular}

FREQUENCIES AND ROTATIONAL CONSTANTS

INDEX NO. DOF TYPE CM-1(UNSCALED) CM-1 (SCALED BY ) SYMMETRY IR-INTENSITY

\begin{tabular}{|c|c|c|c|c|c|}
\hline 1 & vib & 151.15 & 0.00 & A & 0.7785 \\
\hline 2 & vib & 217.95 & 0.00 & A & 1.5118 \\
\hline 3 & vib & 287.28 & 0.00 & A & 0.1958 \\
\hline 4 & vib & 507.04 & 0.00 & A & 0.7013 \\
\hline 5 & vib & 546.25 & 0.00 & A & 5.5876 \\
\hline 6 & vib & 744.75 & 0.00 & A & 0.0552 \\
\hline 7 & vib & 799.15 & 0.00 & A & 49.0327 \\
\hline 8 & vib & 882.69 & 0.00 & A & 6.3745 \\
\hline 9 & vib & 996.67 & 0.00 & A & 4.4384 \\
\hline 10 & vib & 997.21 & 0.00 & A & 22.3936 \\
\hline 11 & vib & 1030.83 & 0.00 & A & 2.6579 \\
\hline 12 & vib & 1140.87 & 0.00 & A & 0.7407 \\
\hline 13 & vib & 1209.35 & 0.00 & A & 0.7917 \\
\hline 14 & vib & 1287.99 & 0.00 & A & 2.0935 \\
\hline 15 & vib & 1348.84 & 0.00 & A & 1.1815 \\
\hline 16 & vib & 1411.96 & 0.00 & A & 1.2829 \\
\hline 17 & vib & 1473.30 & 0.00 & A & 6.0101 \\
\hline 18 & vib & 1477.22 & 0.00 & A & 15.5944 \\
\hline 19 & vib & 1513.03 & 0.00 & A & 7.1215 \\
\hline 20 & vib & 1528.97 & 0.00 & A & 1.3889 \\
\hline 21 & vib & 2994.91 & 0.00 & A & 33.1940 \\
\hline 22 & vib & 3024.43 & 0.00 & A & 21.7679 \\
\hline 23 & vib & 3086.63 & 0.00 & A & 14.4936 \\
\hline 24 & vib & 3113.45 & 0.00 & A & 7.4451 \\
\hline 25 & vib & 3134.03 & 0.00 & A & 24.5439 \\
\hline 26 & vib & 3141.59 & 0.00 & A & 16.5485 \\
\hline 27 & vib & 3234.93 & 0.00 & A & 12.1312 \\
\hline 28 & rot & 1.2823256 & - & & \\
\hline 29 & rot & 0.1338445 & - & & \\
\hline 30 & rot & 0.1239441 & - & & \\
\hline
\end{tabular}

SPIN CONTAMINATION

$\begin{array}{cccccc}\text { Charge } & \text { Multiplicity } & \mathrm{S}^{\wedge} 2 \text { Before } & \mathrm{S}^{\wedge} 2 \text { After } & \mathrm{S}^{\wedge} 2 \text { Expected } & \% \text { Difference? } \\ 0 & 2 & 0.7767 & 0.7502 & 0.7500 & 0.03\end{array}$




$\begin{array}{llcc} & \text { E (Thermal) } & \text { CV } & \text { S } \\ & \text { KCal/Mol } & \text { Cal/Mol-Kelvin } & \text { Cal/Mol-Kelvin } \\ \text { VIBRATIONS ONLY: } & 62.329 & 17.915 & 71.571 \\ \text { HR CORRECTED: } & 62.362 & 17.713 & 72.007 \\ \text { CORRECTED-VIB: } & 0.033 & -0.202 & 0.436\end{array}$

\section{Cis-2-butene.log}

\begin{tabular}{|c|c|c|c|c|c|}
\hline \multirow{2}{*}{$\begin{array}{l}\text { Center } \\
\text { Number }\end{array}$} & \multirow{2}{*}{$\begin{array}{l}\text { Atomic } \\
\text { Number }\end{array}$} & \multirow{2}{*}{$\begin{array}{l}\text { Atomic } \\
\text { Type }\end{array}$} & \multicolumn{3}{|c|}{ Coordinates (Angstroms) } \\
\hline & & & $\mathrm{X}$ & $\mathrm{Y}$ & Z \\
\hline 1 & 6 & 0 & 0.000000 & 0.665679 & 0.661920 \\
\hline 2 & 1 & 0 & 0.000000 & 1.161854 & 1.627754 \\
\hline 3 & 6 & 0 & 0.000000 & -0.665679 & 0.661920 \\
\hline 4 & 1 & 0 & 0.000000 & -1.161854 & 1.627754 \\
\hline 5 & 6 & 0 & 0.000000 & 1.586219 & -0.520638 \\
\hline 6 & 1 & 0 & 0.000000 & 1.056996 & -1.471593 \\
\hline 7 & 1 & 0 & -0.875798 & 2.240653 & -0.501925 \\
\hline 8 & 1 & 0 & 0.875798 & 2.240653 & -0.501925 \\
\hline 9 & 6 & 0 & 0.000000 & -1.586219 & -0.520638 \\
\hline 10 & 1 & 0 & 0.875798 & -2.240653 & -0.501925 \\
\hline 11 & 1 & 0 & -0.875798 & -2.240653 & -0.501925 \\
\hline 12 & 1 & 0 & 0.000000 & -1.056996 & -1.471593 \\
\hline
\end{tabular}

FREQUENCIES AND ROTATIONAL CONSTANTS

\begin{tabular}{|c|c|c|c|c|c|}
\hline INDEX NO. & DOF TYPE & CM-1 (UNSCALED) & CM-1 (SCALED BY ) & SYMMETRY & IR-INTENSITY \\
\hline 1 & vib & 124.67 & 0.00 & B1 & 0.8351 \\
\hline 2 & vib & 136.40 & 0.00 & A2 & 0.0000 \\
\hline 3 & vib & 285.37 & 0.00 & $\mathrm{~A} 1$ & 0.0037 \\
\hline 4 & vib & 401.06 & 0.00 & A2 & 0.0000 \\
\hline 5 & vib & 572.02 & 0.00 & B2 & 6.0818 \\
\hline 6 & vib & 697.81 & 0.00 & B1 & 37.1519 \\
\hline 7 & vib & 868.45 & 0.00 & $\mathrm{~A} 1$ & 0.3946 \\
\hline 8 & vib & 979.09 & 0.00 & B2 & 16.8506 \\
\hline 9 & vib & 1010.78 & 0.00 & A2 & 0.0000 \\
\hline 10 & vib & 1025.88 & 0.00 & $\mathrm{~A} 1$ & 0.9053 \\
\hline 11 & vib & 1066.94 & 0.00 & A2 & 0.0000 \\
\hline 12 & vib & 1067.33 & 0.00 & B1 & 3.3099 \\
\hline 13 & vib & 1158.79 & 0.00 & B2 & 0.3564 \\
\hline 14 & vib & 1296.45 & 0.00 & $\mathrm{~A} 1$ & 0.0135 \\
\hline 15 & vib & 1396.49 & 0.00 & B2 & 1.4212 \\
\hline 16 & vib & 1420.64 & 0.00 & $\mathrm{~A} 1$ & 3.9789 \\
\hline
\end{tabular}




\begin{tabular}{|c|c|c|c|c|c|}
\hline 17 & vib & 1441.93 & 0.00 & B2 & 10.1336 \\
\hline 18 & vib & 1482.40 & 0.00 & A2 & 0.0000 \\
\hline 19 & vib & 1486.51 & 0.00 & B1 & 11.1830 \\
\hline 20 & vib & 1490.62 & 0.00 & B2 & 3.9923 \\
\hline 21 & vib & 1495.95 & 0.00 & A1 & 5.1493 \\
\hline 22 & vib & 1731.33 & 0.00 & A1 & 4.7329 \\
\hline 23 & vib & 3014.68 & 0.00 & B2 & 46.1674 \\
\hline 24 & vib & 3016.94 & 0.00 & A1 & 11.8078 \\
\hline 25 & vib & 3050.93 & 0.00 & A2 & 0.0000 \\
\hline 26 & vib & 3051.28 & 0.00 & B1 & 44.3726 \\
\hline 27 & vib & 3096.47 & 0.00 & B2 & 5.2007 \\
\hline 28 & vib & 3111.24 & 0.00 & A1 & 12.7404 \\
\hline 29 & vib & 3118.49 & 0.00 & B2 & 15.8685 \\
\hline 30 & vib & 3140.68 & 0.00 & A1 & 53.3773 \\
\hline 31 & rot & 0.5476143 & - & & \\
\hline 32 & rot & 0.1697210 & - & & \\
\hline 33 & rot & 0.1360308 & - & & \\
\hline
\end{tabular}

\section{6 cis-HCOO.log}

\section{CNCN-freqs.log}

Charge $=0$ Multiplicity $=1$ Stoichiometry $\quad$ C2N2

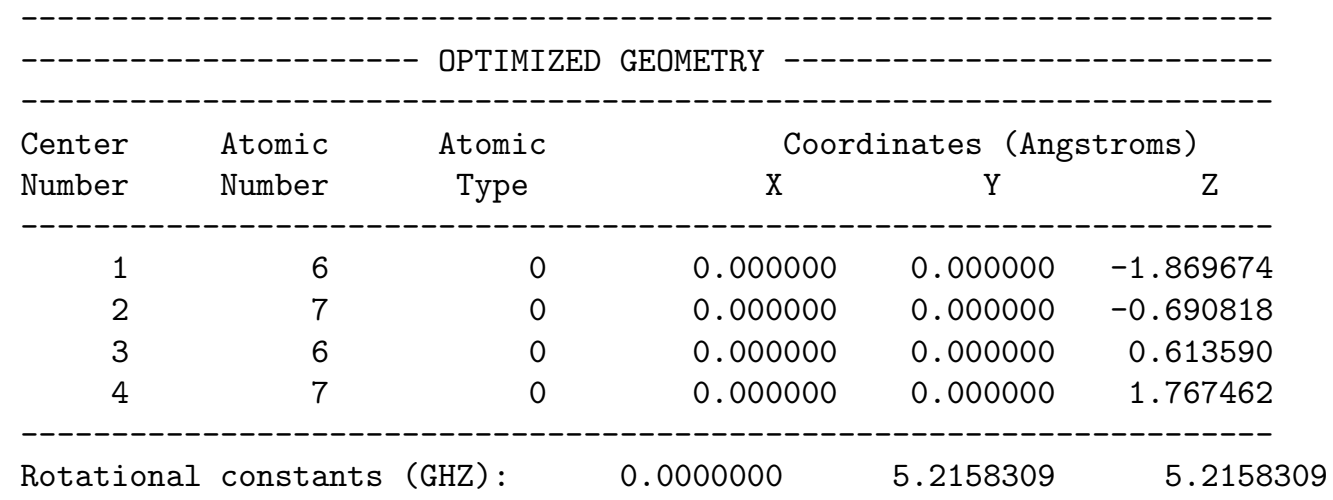

FREQUENCIES AND ROTATIONAL CONSTANTS

$\begin{array}{cccccr}\text { INDEX NO. DOF TYPE } & \text { CM-1(UNSCALED) } & \text { CM-1 (SCALED BY }) & \text { SYMMETRY IR-INTENSITY } \\ 1 & \text { vib } & 215.38 & 0.00 & \text { PI } & 9.3177 \\ 2 & \text { vib } & 215.38 & 0.00 & \text { PI } & 9.3177 \\ 3 & \text { vib } & 508.83 & 0.00 & \text { PI } & 7.3619 \\ 4 & \text { vib } & 508.83 & 0.00 & \text { PI } & 7.3619 \\ 5 & \text { vib } & 960.61 & 0.00 & \text { SG } & 0.6281 \\ 6 & \text { vib } & 2133.30 & 0.00 & \text { SG } & 156.4061 \\ 7 & \text { vib } & 2393.22 & 0.00 & \text { SG } & 107.5784 \\ 8 & \text { vib } & 0.00 & 0.00 & & \\ 9 & \text { vib } & 0.00 & 0.00 & & \end{array}$




$\begin{array}{llll}10 & \text { rot } & 0.0000000 & - \\ 11 & \text { rot } & 0.1739814 & - \\ 12 & \text { rot } & 0.1739814 & -\end{array}$

\section{CNN-triplet-freqs.log}

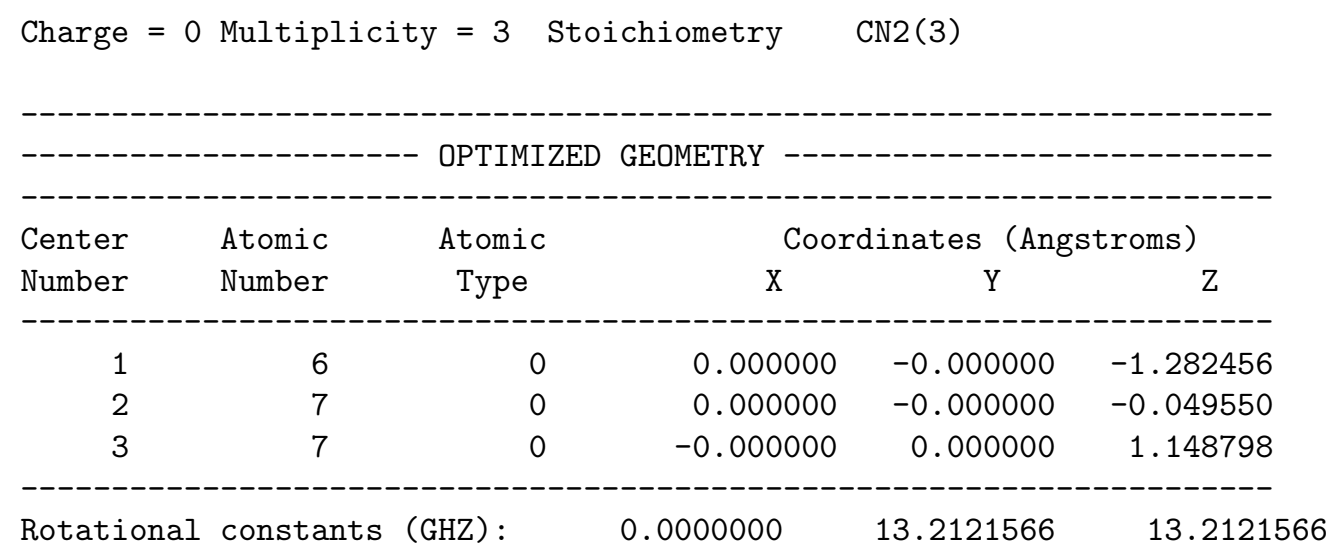

FREQUENCIES AND ROTATIONAL CONSTANTS

\begin{tabular}{cccccr} 
INDEX NO. DOF TYPE & CM-1(UNSCALED) & CM-1 (SCALED BY ) & SYMMETRY IR-INTENSITY \\
1 & vib & 408.76 & 0.00 & PI & 7.2837 \\
2 & vib & 408.76 & 0.00 & PI & 7.2837 \\
3 & vib & 1284.93 & 0.00 & SG & 15.6286 \\
4 & vib & 1511.75 & 0.00 & SG & 0.6175 \\
5 & vib & 0.00 & 0.00 & \\
6 & vib & 0.00 & 0.00 & \\
7 & rot & 0.0000000 & - & \\
8 & rot & 0.4407101 & - & & \\
9 & rot & 0.4407101 & - & & \\
\hline
\end{tabular}

\begin{tabular}{|c|c|c|c|c|c|}
\hline Charge & Multiplicity & $\mathrm{S}^{\wedge} 2$ Before & $S^{\wedge} 2$ After & $S^{\wedge} 2$ Expected & $\%$ Difference? \\
\hline 0 & 3 & 2.0347 & 2.0006 & 2.0000 & 0.03 \\
\hline
\end{tabular}

\section{CNNC-freqs.log}

Charge $=0$ Multiplicity $=1$ Stoichiometry $\quad$ C2N2

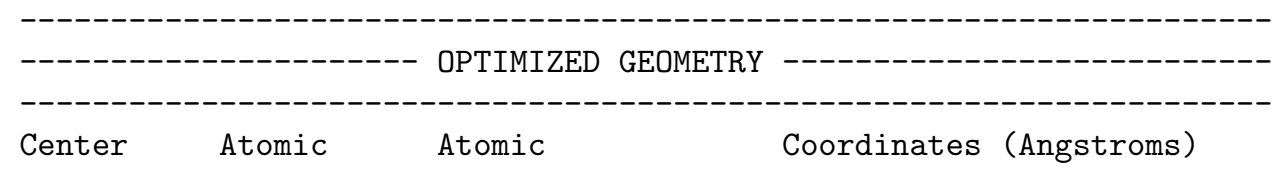




\begin{tabular}{|c|c|c|c|c|c|c|}
\hline Number & Number & Type & $\mathrm{X}$ & $\mathrm{Y}$ & \multicolumn{2}{|c|}{$\mathrm{Z}$} \\
\hline 1 & 6 & 0 & 0.000000 & 0.000000 & \multicolumn{2}{|c|}{1.812605} \\
\hline 2 & 7 & 0 & 0.000000 & 0.000000 & \multicolumn{2}{|c|}{0.635704} \\
\hline 3 & 7 & 0 & 0.000000 & 0.000000 & \multicolumn{2}{|c|}{-0.635704} \\
\hline 4 & 6 & 0 & 0.000000 & 0.000000 & \multicolumn{2}{|c|}{-1.812605} \\
\hline Rotational & constants & (GHZ): $\quad 0$ & 0000000 & 5.6046925 & \multicolumn{2}{|c|}{5.6046925} \\
\hline \multicolumn{7}{|c|}{ 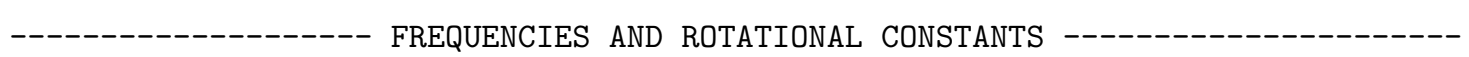 } \\
\hline INDEX NO. I & DOF TYPE & CM-1 (UNSCALED) & CM-1 (SCALED & BY ) SYMME & RY I & NTENSITY \\
\hline 1 & vib & 204.11 & 0.00 & & PIU & 5.4224 \\
\hline 2 & vib & 204.11 & 0.00 & & PIU & 5.4224 \\
\hline 3 & vib & 366.43 & 0.00 & & PIG & 0.0000 \\
\hline 4 & vib & 366.43 & 0.00 & & PIG & 0.0000 \\
\hline 5 & vib & 977.01 & 0.00 & & SGG & 0.0000 \\
\hline 6 & vib & 2076.53 & 0.00 & & SGU & 273.7713 \\
\hline 7 & vib & 2288.82 & 0.00 & & SGG & 0.0000 \\
\hline 8 & vib & 0.00 & 0.00 & & & \\
\hline 9 & vib & 0.00 & 0.00 & & & \\
\hline 10 & rot & 0.0000000 & - & & & \\
\hline 11 & rot & 0.1869524 & - & & & \\
\hline 12 & rot & 0.1869524 & - & & & \\
\hline
\end{tabular}

\section{CPO-C2symm-freqs.log}

Charge $=0$ Multiplicity $=1$ Stoichiometry $\quad$ C5H80

\begin{tabular}{|c|c|c|c|c|c|}
\hline \multirow{2}{*}{$\begin{array}{l}\text { Center } \\
\text { Number }\end{array}$} & \multirow{2}{*}{$\begin{array}{l}\text { Atomic } \\
\text { Number }\end{array}$} & \multirow{2}{*}{$\begin{array}{c}\text { Atomic } \\
\text { Type }\end{array}$} & \multicolumn{3}{|c|}{ Coordinates (Angstroms) } \\
\hline & & & $\mathrm{X}$ & $\mathrm{Y}$ & $\mathrm{Z}$ \\
\hline 1 & 8 & 0 & -0.000000 & 0.000000 & 2.129618 \\
\hline 2 & 6 & 0 & -0.000000 & 0.000000 & 0.925476 \\
\hline 3 & 6 & 0 & -0.000000 & 1.239405 & 0.030965 \\
\hline 4 & 6 & 0 & -0.000000 & -1.239405 & 0.030965 \\
\hline 5 & 6 & 0 & 0.294692 & 0.713283 & -1.378400 \\
\hline 6 & 6 & 0 & -0.294692 & -0.713283 & -1.378400 \\
\hline 7 & 1 & 0 & -1.002042 & 1.676468 & 0.088892 \\
\hline 8 & 1 & 0 & 1.002042 & -1.676468 & 0.088892 \\
\hline 9 & 1 & 0 & 0.695855 & 1.987709 & 0.406929 \\
\hline 10 & 1 & 0 & -0.695855 & -1.987709 & 0.406929 \\
\hline 11 & 1 & 0 & -0.122150 & 1.338698 & -2.166349 \\
\hline 12 & 1 & 0 & 0.122150 & -1.338698 & -2.166349 \\
\hline 13 & 1 & 0 & 1.374237 & 0.665728 & -1.539762 \\
\hline 14 & 1 & 0 & -1.374237 & -0.665728 & -1.539762 \\
\hline
\end{tabular}


FREQUENCIES AND ROTATIONAL CONSTANTS

\begin{tabular}{|c|c|c|c|c|c|}
\hline INDEX NO. & DOF TYPE & CM-1 (UNSCALED) & CM-1 (SCALED BY ) & SYMMETRY I & TENSITY \\
\hline 1 & vib & 95.84 & 0.00 & B & 4.9097 \\
\hline 2 & vib & 229.24 & 0.00 & A & 0.1143 \\
\hline 3 & vib & 452.00 & 0.00 & B & 3.3996 \\
\hline 4 & vib & 472.36 & 0.00 & B & 4.6460 \\
\hline 5 & vib & 569.32 & 0.00 & A & 5.2306 \\
\hline 6 & vib & 589.03 & 0.00 & B & 2.5867 \\
\hline 7 & vib & 707.61 & 0.00 & A & 1.3351 \\
\hline 8 & vib & 809.25 & 0.00 & A & 2.0067 \\
\hline 9 & vib & 841.98 & 0.00 & B & 10.6319 \\
\hline 10 & vib & 893.65 & 0.00 & A & 0.3776 \\
\hline 11 & vib & 925.79 & 0.00 & B & 0.9150 \\
\hline 12 & vib & 964.49 & 0.00 & A & 0.1221 \\
\hline 13 & vib & 968.59 & 0.00 & B & 10.0722 \\
\hline 14 & vib & 1030.70 & 0.00 & A & 0.5261 \\
\hline 15 & vib & 1147.54 & 0.00 & B & 76.6139 \\
\hline 16 & vib & 1169.03 & 0.00 & B & 2.2842 \\
\hline 17 & vib & 1173.58 & 0.00 & A & 0.5188 \\
\hline 18 & vib & 1222.20 & 0.00 & A & 0.0835 \\
\hline 19 & vib & 1256.18 & 0.00 & B & 1.2865 \\
\hline 20 & vib & 1298.78 & 0.00 & B & 5.0157 \\
\hline 21 & vib & 1308.42 & 0.00 & A & 1.6414 \\
\hline 22 & vib & 1342.22 & 0.00 & A & 0.1339 \\
\hline 23 & vib & 1346.50 & 0.00 & B & 2.6368 \\
\hline 24 & vib & 1452.72 & 0.00 & B & 19.3251 \\
\hline 25 & vib & 1453.84 & 0.00 & A & 0.0193 \\
\hline 26 & vib & 1500.34 & 0.00 & B & 4.3490 \\
\hline 27 & vib & 1512.64 & 0.00 & A & 1.6266 \\
\hline 28 & vib & 1817.66 & 0.00 & A & 240.1323 \\
\hline 29 & vib & 3022.44 & 0.00 & A & 1.4544 \\
\hline 30 & vib & 3022.64 & 0.00 & B & 5.7106 \\
\hline 31 & vib & 3029.94 & 0.00 & A & 24.2247 \\
\hline 32 & vib & 3037.13 & 0.00 & B & 41.8000 \\
\hline 33 & vib & 3086.21 & 0.00 & A & 24.9744 \\
\hline 34 & vib & 3090.20 & 0.00 & B & 31.7642 \\
\hline 35 & vib & 3098.94 & 0.00 & A & 5.9559 \\
\hline 36 & vib & 3099.60 & 0.00 & B & 25.2632 \\
\hline 37 & rot & 0.2214240 & - & & \\
\hline 38 & rot & 0.1117387 & - & & \\
\hline 39 & rot & 0.0801354 & - & & \\
\hline
\end{tabular}

\section{CPO-freqs-z-matrix.log}

Charge $=0$ Multiplicity $=1 \quad$ Stoichiometry $\quad$ C5H8O 


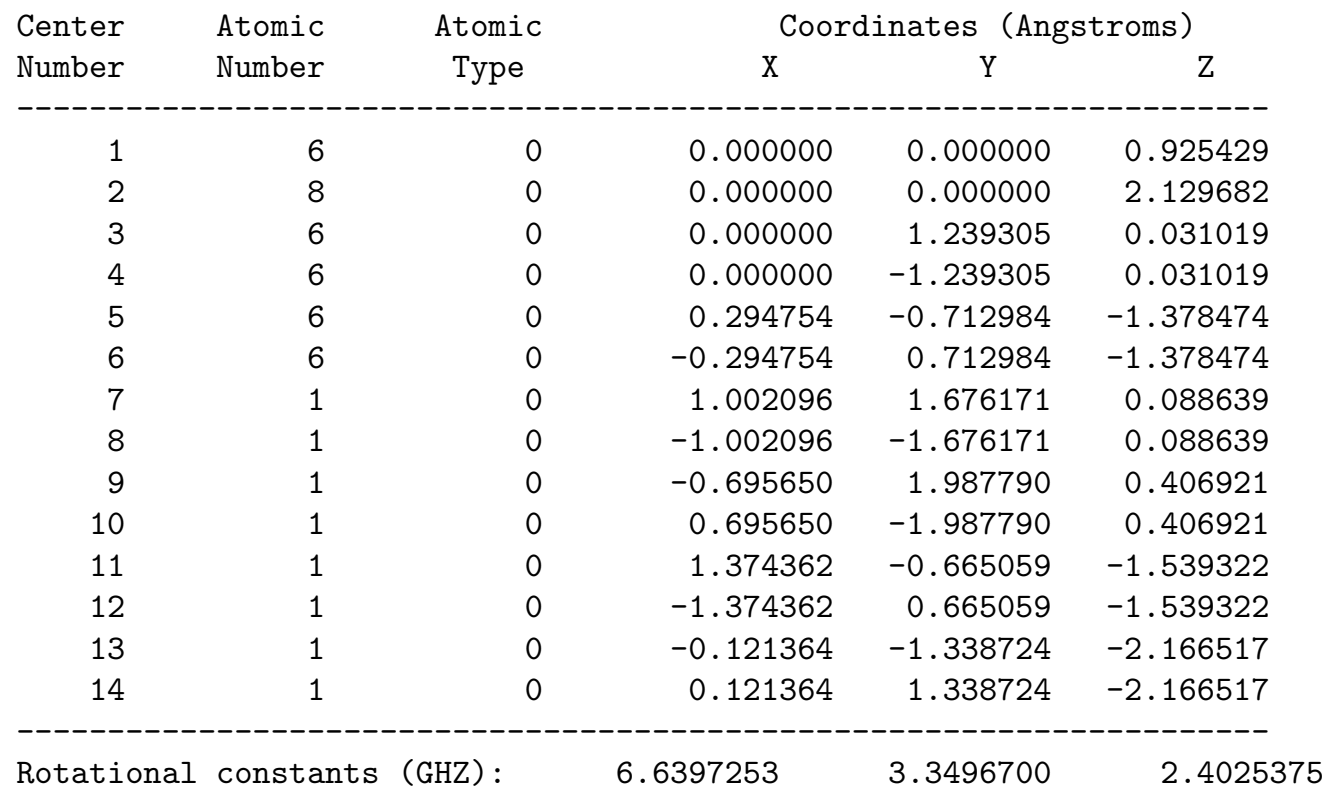

FREQUENCIES AND ROTATIONAL CONSTANTS

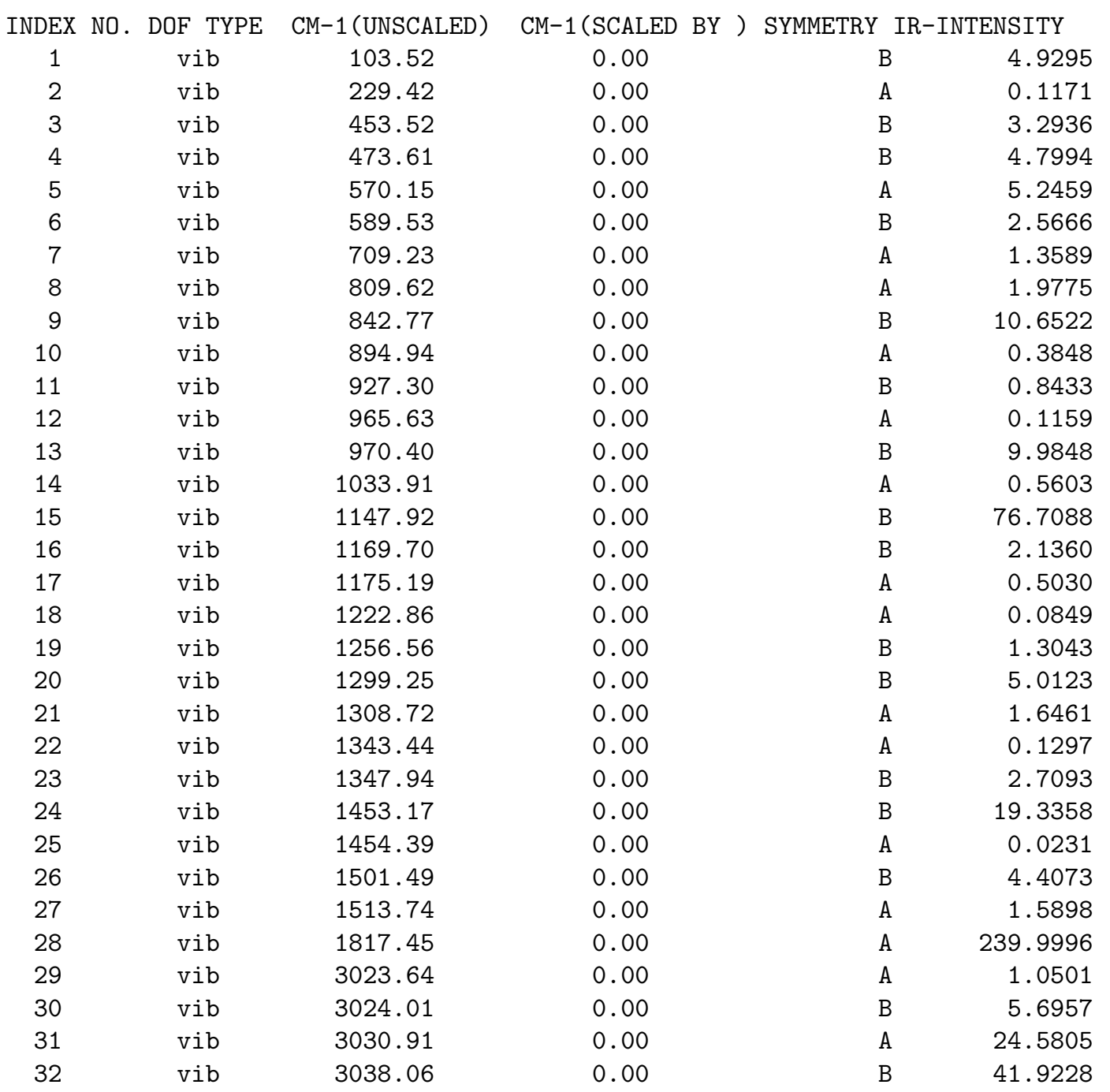




$\begin{array}{lllllr}33 & \text { vib } & 3087.22 & 0.00 & \text { A } & 24.9920 \\ 34 & \text { vib } & 3091.18 & 0.00 & \text { B } & 31.7453 \\ 35 & \text { vib } & 3099.91 & 0.00 & \text { A } & 5.9448 \\ 36 & \text { vib } & 3100.54 & 0.00 & \text { B } & 25.2120 \\ 37 & \text { rot } & 0.2214774 & - & & \\ 38 & \text { rot } & 0.1117330 & - & \\ 39 & \text { rot } & 0.0801400 & - & \end{array}$

\section{Cyanato.log}

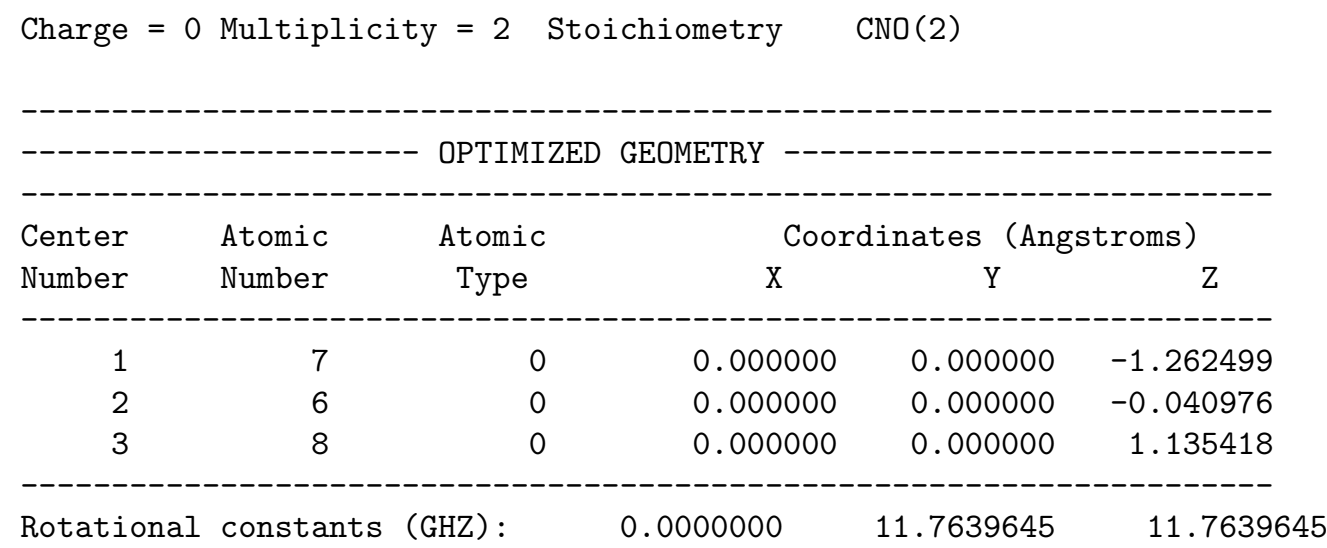

\section{FREQUENCIES AND ROTATIONAL CONSTANTS}

INDEX NO. DOF TYPE CM-1(UNSCALED) CM-1(SCALED BY) SYMMETRY IR-INTENSITY

$\begin{array}{rrrrrr}1 & \text { vib } & 509.70 & 0.00 & \text { PI } & 38.2358 \\ 2 & \text { vib } & 591.88 & 0.00 & \text { PI } & 15.0626 \\ 3 & \text { vib } & 1297.49 & 0.00 & \text { SG } & 8.7016 \\ 4 & \text { vib } & 1990.56 & 0.00 & \text { SG } & 64.1135 \\ 5 & \text { vib } & 0.00 & 0.00 & & \\ 6 & \text { vib } & 0.00 & 0.00 & \\ 7 & \text { rot } & 0.0000000 & - & \\ 8 & \text { rot } & 0.3924036 & - & \\ 9 & \text { rot } & 0.3924036 & - & \end{array}$

SPIN CONTAMINATION

\begin{tabular}{|c|c|c|c|c|c|}
\hline Charge & Multiplicity & $\mathrm{S}^{\wedge} 2$ Before & $\mathrm{S}^{\wedge} 2$ After & $S^{\wedge} 2$ Expected & $\%$ Difference? \\
\hline 0 & 2 & 0.7624 & 0.7501 & 0.7500 & 0.01 \\
\hline
\end{tabular}

\section{CyanicAcid.log}

Charge $=0$ Multiplicity $=1$ Stoichiometry CHNO 


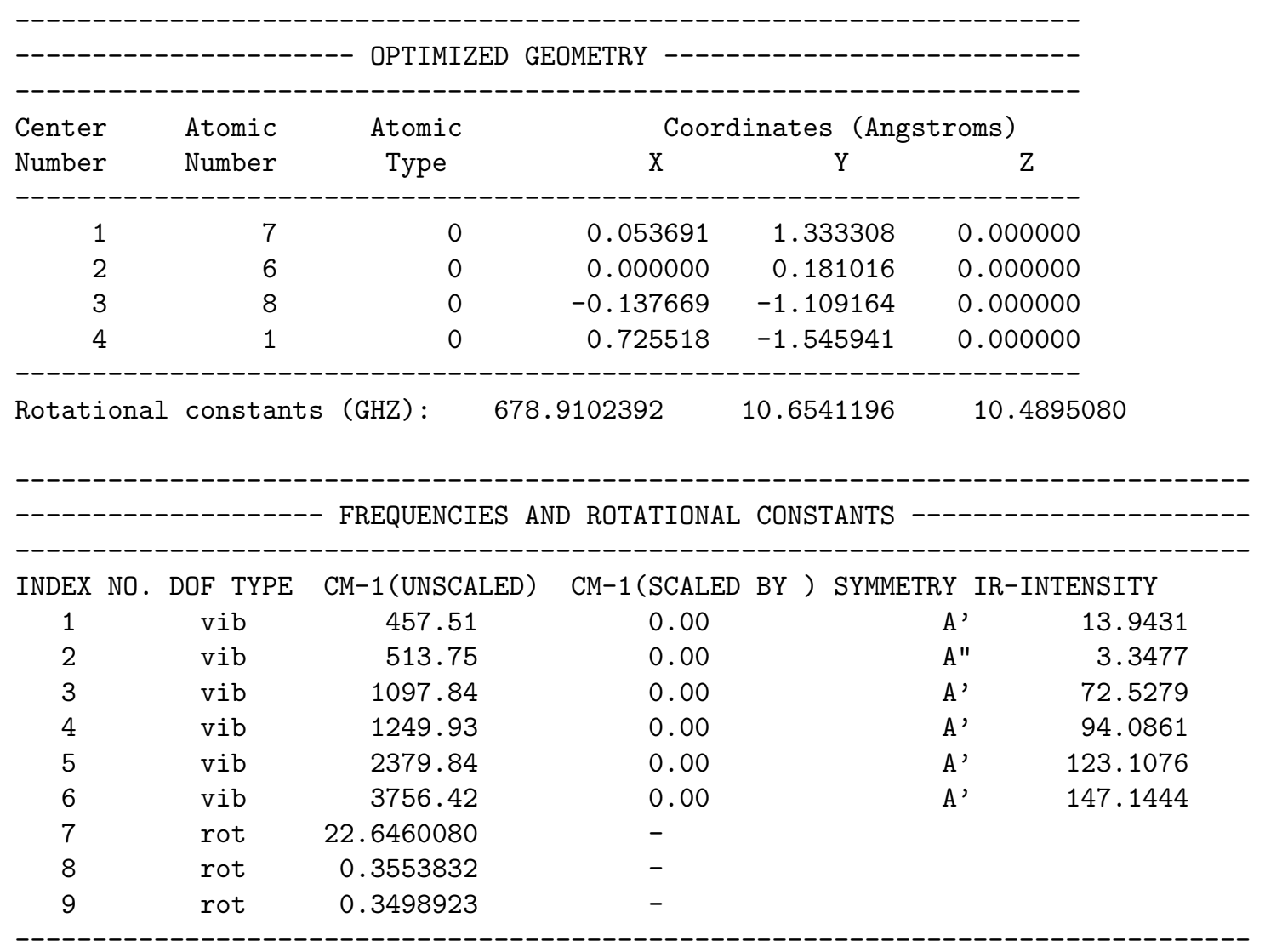

\section{Cyanogen-anharmonic.log}

Charge $=0$ Multiplicity $=1$ Stoichiometry $\quad$ C2N2

\begin{tabular}{|c|c|c|c|c|c|}
\hline \multirow{2}{*}{$\begin{array}{l}\text { Center } \\
\text { Number }\end{array}$} & \multirow{2}{*}{$\begin{array}{l}\text { Atomic } \\
\text { Number }\end{array}$} & \multirow{2}{*}{$\begin{array}{c}\text { Atomic } \\
\text { Type }\end{array}$} & \multicolumn{3}{|c|}{ Coordinates (Angstroms) } \\
\hline & & & $\mathrm{X}$ & Y & Z \\
\hline 1 & 6 & 0 & 0.000000 & 0.000000 & -0.687898 \\
\hline 2 & 7 & 0 & 0.000000 & 0.000000 & -1.845972 \\
\hline 3 & 6 & 0 & 0.000000 & 0.000000 & 0.687896 \\
\hline 4 & 7 & 0 & 0.000000 & 0.000000 & 1.845974 \\
\hline Rotatic & & HZ) & 000000 & 4092 & 4.73240 \\
\hline
\end{tabular}

FREQUENCIES AND ROTATIONAL CONSTANTS

$\begin{array}{ccccrr}\text { INDEX NO. DOF TYPE } & \text { CM-1(UNSCALED) } & \text { CM-1(SCALED BY } 1 \text { ) } & \text { SYMMETRY } & \text { IR-INTENSITY } \\ 1 & \text { vib } & 261.18 & 261.18 & \text { PI } & 21.5451 \\ 2 & \text { vib } & 261.18 & 261.18 & \text { PI } & 21.5451 \\ 3 & \text { vib } & 576.10 & 576.10 & \text { PI } & 0.0000 \\ 4 & \text { vib } & 576.10 & 576.10 & \text { PI } & 0.0000\end{array}$




$\begin{array}{rrrrrr}5 & \text { vib } & 886.30 & 886.30 & \text { SG } & 0.0000 \\ 6 & \text { vib } & 2268.62 & 2268.62 & \text { SG } & 0.0010 \\ 7 & \text { vib } & 2433.18 & 2433.18 & \text { SG } & 0.0000 \\ 8 & \text { vib } & 0.00 & 0.00 & & \\ 9 & \text { vib } & 0.00 & 0.00 & \text { PI } & 21.5451 \\ 10 & \text { vib } & 261.18 & 261.18 & \text { PI } & 21.5451 \\ 11 & \text { vib } & 261.18 & 261.18 & \text { PI } & 0.0000 \\ 12 & \text { vib } & 576.10 & 576.10 & \text { PI } & 0.0000 \\ 13 & \text { vib } & 576.10 & 576.10 & \text { SG } & 0.0000 \\ 14 & \text { vib } & 886.30 & 886.30 & \text { SG } & 0.0010 \\ 15 & \text { vib } & 2268.62 & 2268.62 & \text { SG } & 0.0000 \\ 16 & \text { vib } & 2433.18 & 2433.18 & & \\ 17 & \text { vib } & 0.00 & 0.00 & & \\ 18 & \text { vib } & 0.00 & 0.00 & & \\ 19 & \text { rot } & 0.0000000 & - & & \\ 20 & \text { rot } & 0.1578562 & - & & \end{array}$

\section{Cyclobutanone-freqs.log}

Charge $=0$ Multiplicity $=1$ Stoichiometry $\quad$ C4H60

\begin{tabular}{|c|c|c|c|c|c|}
\hline \multirow{2}{*}{$\begin{array}{l}\text { Center } \\
\text { Number }\end{array}$} & \multirow{2}{*}{$\begin{array}{l}\text { Atomic } \\
\text { Number }\end{array}$} & \multirow{2}{*}{$\begin{array}{c}\text { Atomic } \\
\text { Type }\end{array}$} & \multicolumn{3}{|c|}{ Coordinates (Angstroms) } \\
\hline & & & $\mathrm{X}$ & $\mathrm{Y}$ & $\mathrm{Z}$ \\
\hline 1 & 8 & 0 & 0.145861 & 1.867526 & 0.000000 \\
\hline 2 & 6 & 0 & 0.032678 & 0.677189 & 0.000000 \\
\hline 3 & 6 & 0 & -0.051487 & -0.380525 & 1.107918 \\
\hline 4 & 6 & 0 & -0.051487 & -0.380525 & -1.107918 \\
\hline 5 & 6 & 0 & -0.051487 & -1.475155 & -0.000000 \\
\hline 6 & 1 & 0 & -0.924836 & -2.122324 & -0.000000 \\
\hline 7 & 1 & 0 & 0.841525 & -2.095538 & -0.000000 \\
\hline 8 & 1 & 0 & 0.793408 & -0.376371 & 1.796859 \\
\hline 9 & 1 & 0 & 0.793408 & -0.376371 & -1.796859 \\
\hline 10 & 1 & 0 & -0.969849 & -0.307751 & 1.692274 \\
\hline 11 & 1 & 0 & -0.969849 & -0.307751 & -1.692274 \\
\hline
\end{tabular}

FREQUENCIES AND ROTATIONAL CONSTANTS

INDEX NO. DOF TYPE CM-1(UNSCALED) CM-1 (SCALED BY ) SYMMETRY IR-INTENSITY

$\begin{array}{llllll}1 & \text { vib } & 29.18 & 0.00 & A^{\prime} & 3.9269 \\ 2 & \text { vib } & 403.30 & 0.00 & A^{\prime} & 1.2891 \\ 3 & \text { vib } & 458.68 & 0.00 & A^{\prime \prime} & 3.0853 \\ 4 & \text { vib } & 625.22 & 0.00 & A^{\prime \prime} & 0.0126 \\ 5 & \text { vib } & 673.25 & 0.00 & A^{\prime} & 0.8154 \\ 6 & \text { vib } & 740.15 & 0.00 & A^{\prime} & 3.3322\end{array}$




\begin{tabular}{|c|c|c|c|c|c|}
\hline 7 & vib & 835.94 & 0.00 & $A^{\prime}$ & 3.3956 \\
\hline 8 & vib & 916.77 & 0.00 & A" & 0.3299 \\
\hline 9 & vib & 946.35 & 0.00 & A" & 0.0421 \\
\hline 10 & vib & 964.78 & 0.00 & $A^{\prime}$ & 2.4373 \\
\hline 11 & vib & 1079.43 & 0.00 & A" & 87.3778 \\
\hline 12 & vib & 1098.12 & 0.00 & $A^{\prime}$ & 0.1249 \\
\hline 13 & vib & 1188.16 & 0.00 & A" & 1.4645 \\
\hline 14 & vib & 1222.45 & 0.00 & $A^{\prime}$ & 0.1314 \\
\hline 15 & vib & 1226.54 & 0.00 & A" & 0.1536 \\
\hline 16 & vib & 1238.65 & 0.00 & $A^{\prime}$ & 4.5376 \\
\hline 17 & vib & 1273.14 & 0.00 & A" & 6.8740 \\
\hline 18 & vib & 1429.49 & 0.00 & A" & 19.3554 \\
\hline 19 & vib & 1445.02 & 0.00 & $A^{\prime}$ & 0.9661 \\
\hline 20 & vib & 1499.67 & 0.00 & $A^{\prime}$ & 0.9951 \\
\hline 21 & vib & 1866.24 & 0.00 & $A^{\prime}$ & 309.8238 \\
\hline 22 & vib & 3050.81 & 0.00 & A" & 16.2182 \\
\hline 23 & vib & 3055.02 & 0.00 & $A^{\prime}$ & 8.2468 \\
\hline 24 & vib & 3079.54 & 0.00 & $A^{\prime}$ & 24.6040 \\
\hline 25 & vib & 3094.09 & 0.00 & $A^{\prime}$ & 4.2464 \\
\hline 26 & vib & 3100.43 & 0.00 & A" & 0.6831 \\
\hline 27 & vib & 3128.18 & 0.00 & $A^{\prime}$ & 26.7756 \\
\hline 28 & rot & 0.3623279 & - & & \\
\hline 29 & rot & 0.1604468 & - & & \\
\hline 30 & rot & 0.1186983 & - & & \\
\hline
\end{tabular}

\section{Cyclohexane-chair-D3d.log}

Charge $=0$ Multiplicity $=1$ Stoichiometry C6H12

\begin{tabular}{|c|c|c|c|c|c|}
\hline \multirow{2}{*}{$\begin{array}{l}\text { Center } \\
\text { Number }\end{array}$} & \multirow{2}{*}{$\begin{array}{l}\text { Atomic } \\
\text { Number }\end{array}$} & \multirow{2}{*}{$\begin{array}{c}\text { Atomic } \\
\text { Type }\end{array}$} & \multicolumn{3}{|c|}{ Coordinates (Angstroms) } \\
\hline & & & $\mathrm{X}$ & Y & $\mathrm{Z}$ \\
\hline 1 & 6 & 0 & 0.000000 & -1.463408 & 0.227399 \\
\hline 2 & 6 & 0 & -1.267349 & 0.731704 & 0.227399 \\
\hline 3 & 6 & 0 & 1.267349 & 0.731704 & 0.227399 \\
\hline 4 & 6 & 0 & 0.000000 & 1.463408 & -0.227399 \\
\hline 5 & 6 & 0 & -1.267349 & -0.731704 & -0.227399 \\
\hline 6 & 6 & 0 & 1.267349 & -0.731704 & -0.227399 \\
\hline 7 & 1 & 0 & 0.000000 & -1.535161 & 1.320446 \\
\hline 8 & 1 & 0 & -1.329488 & 0.767581 & 1.320446 \\
\hline 9 & 1 & 0 & 1.329488 & 0.767580 & 1.320446 \\
\hline 10 & 1 & 0 & 0.000000 & 1.535161 & -1.320446 \\
\hline 11 & 1 & 0 & -1.329488 & -0.767580 & -1.320446 \\
\hline 12 & 1 & 0 & 1.329488 & -0.767581 & -1.320446 \\
\hline 13 & 1 & 0 & 0.000000 & -2.488680 & -0.149734 \\
\hline 14 & 1 & 0 & -2.155260 & 1.244340 & -0.149734 \\
\hline 15 & 1 & 0 & 2.155260 & 1.244340 & -0.149734 \\
\hline 16 & 1 & 0 & 0.000000 & 2.488680 & 0.149734 \\
\hline 17 & 1 & 0 & -2.155260 & -1.244340 & 0.149734 \\
\hline
\end{tabular}




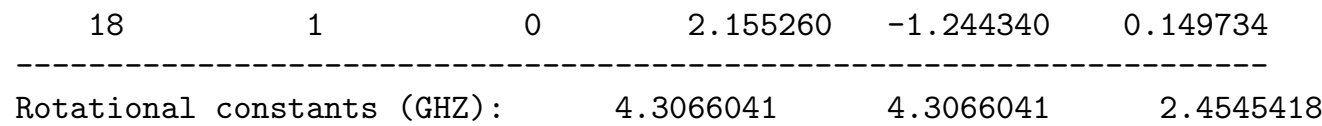

FREQUENCIES AND ROTATIONAL CONSTANTS

INDEX NO. DOF TYPE CM-1(UNSCALED) CM-1(SCALED BY ) SYMMETRY IR-INTENSITY

\begin{tabular}{|c|c|c|c|c|}
\hline vib & 228.23 & 0.00 & EU & 0.0035 \\
\hline vib & 228.23 & 0.00 & EU & 0.0035 \\
\hline vib & 375.83 & 0.00 & $\mathrm{~A} 1 \mathrm{G}$ & 0.0000 \\
\hline vib & 428.82 & 0.00 & EG & 0.0000 \\
\hline vib & 428.82 & 0.00 & EG & 0.0000 \\
\hline vib & 522.73 & 0.00 & $\mathrm{~A} 2 \mathrm{U}$ & 0.7190 \\
\hline vib & 793.41 & 0.00 & EG & 0.0000 \\
\hline vib & 793.41 & 0.00 & EG & 0.0000 \\
\hline vib & 800.73 & 0.00 & $\mathrm{~A} 1 \mathrm{G}$ & 0.0000 \\
\hline vib & 862.76 & 0.00 & $\mathrm{EU}$ & 1.8509 \\
\hline vib & 862.76 & 0.00 & EU & 1.8512 \\
\hline vib & 918.94 & 0.00 & EU & 1.8035 \\
\hline vib & 918.94 & 0.00 & EU & 1.8035 \\
\hline vib & 1035.83 & 0.00 & EG & 0.0000 \\
\hline vib & 1035.83 & 0.00 & EG & 0.0000 \\
\hline vib & 1045.27 & 0.00 & $\mathrm{~A} 2 \mathrm{U}$ & 2.1941 \\
\hline vib & 1074.70 & 0.00 & $\mathrm{~A} 2 \mathrm{G}$ & 0.0000 \\
\hline vib & 1093.92 & 0.00 & $\mathrm{~A} 1 \mathrm{U}$ & 0.0000 \\
\hline vib & 1130.49 & 0.00 & $\mathrm{~A} 1 \mathrm{U}$ & 0.0000 \\
\hline vib & 1185.12 & 0.00 & $\mathrm{~A} 1 \mathrm{G}$ & 0.0000 \\
\hline vib & 1288.15 & 0.00 & $\mathrm{EU}$ & 1.8728 \\
\hline vib & 1288.15 & 0.00 & $\mathrm{EU}$ & 1.8729 \\
\hline vib & 1293.00 & 0.00 & EG & 0.0000 \\
\hline vib & 1293.00 & 0.00 & EG & 0.0000 \\
\hline vib & 1356.01 & 0.00 & A2G & 0.0000 \\
\hline vib & 1370.46 & 0.00 & $\mathrm{~A} 1 \mathrm{U}$ & 0.0000 \\
\hline vib & 1377.99 & 0.00 & EG & 0.0000 \\
\hline vib & 1377.99 & 0.00 & EG & 0.0000 \\
\hline vib & 1388.98 & 0.00 & EU & 0.3002 \\
\hline vib & 1388.98 & 0.00 & EU & 0.3002 \\
\hline vib & 1486.60 & 0.00 & EG & 0.0000 \\
\hline vib & 1486.60 & 0.00 & EG & 0.0000 \\
\hline vib & 1491.13 & 0.00 & EU & 4.9459 \\
\hline vib & 1491.13 & 0.00 & EU & 4.9463 \\
\hline vib & 1497.41 & 0.00 & $\mathrm{~A} 2 \mathrm{U}$ & 13.3102 \\
\hline vib & 1511.60 & 0.00 & $\mathrm{~A} 1 \mathrm{G}$ & 0.0000 \\
\hline vib & 2995.84 & 0.00 & EU & 24.5741 \\
\hline vib & 2995.84 & 0.00 & $\mathrm{EU}$ & 24.5742 \\
\hline vib & 2996.47 & 0.00 & $\mathrm{~A} 1 \mathrm{G}$ & 0.0000 \\
\hline vib & 3004.09 & 0.00 & EG & 0.0000 \\
\hline vib & 3004.09 & 0.00 & EG & 0.0000 \\
\hline vib & 3007.46 & 0.00 & $\mathrm{~A} 2 \mathrm{U}$ & 74.1831 \\
\hline vib & 3045.31 & 0.00 & $\mathrm{EU}$ & 123.4722 \\
\hline vib & 3045.31 & 0.00 & $\mathrm{EU}$ & 123.4711 \\
\hline vib & 3046.96 & 0.00 & EG & 0.0000 \\
\hline vib & 3046.96 & 0.00 & EG & 0.0000 \\
\hline vib & 3049.34 & 0.00 & $\mathrm{~A} 1 \mathrm{G}$ & 0.0000 \\
\hline vib & 3054.69 & 0.00 & $\mathrm{~A} 2 \mathrm{U}$ & 148.3218 \\
\hline
\end{tabular}




\begin{tabular}{llll}
49 & rot & 0.1436528 & - \\
50 & rot & 0.1436528 & - \\
51 & rot & 0.0818747 & - \\
\hline
\end{tabular}

\section{Cyclohexane-chair-freqs.log}

\begin{tabular}{|c|c|c|c|c|c|}
\hline \multirow{2}{*}{$\begin{array}{l}\text { Center } \\
\text { Number }\end{array}$} & \multirow{2}{*}{$\begin{array}{l}\text { Atomic } \\
\text { Number }\end{array}$} & \multirow{2}{*}{$\begin{array}{c}\text { Atomic } \\
\text { Type }\end{array}$} & \multicolumn{3}{|c|}{ Coordinates (Angstroms) } \\
\hline & & & $\mathrm{X}$ & Y & Z \\
\hline 1 & 6 & 0 & 0.000000 & -1.463360 & 0.227411 \\
\hline 2 & 6 & 0 & -1.267307 & 0.731680 & 0.227411 \\
\hline 3 & 6 & 0 & 1.267307 & 0.731680 & 0.227411 \\
\hline 4 & 6 & 0 & 0.000000 & 1.463360 & -0.227411 \\
\hline 5 & 6 & 0 & -1.267307 & -0.731680 & -0.227411 \\
\hline 6 & 6 & 0 & 1.267307 & -0.731680 & -0.227411 \\
\hline 7 & 1 & 0 & 0.000000 & -1.535260 & 1.320477 \\
\hline 8 & 1 & 0 & -1.329575 & 0.767630 & 1.320477 \\
\hline 9 & 1 & 0 & 1.329575 & 0.767630 & 1.320477 \\
\hline 10 & 1 & 0 & 0.000000 & 1.535260 & -1.320477 \\
\hline 11 & 1 & 0 & -1.329575 & -0.767630 & -1.320477 \\
\hline 12 & 1 & 0 & 1.329575 & -0.767630 & -1.320477 \\
\hline 13 & 1 & 0 & 0.000000 & -2.488618 & -0.149812 \\
\hline 14 & 1 & 0 & -2.155206 & 1.244309 & -0.149812 \\
\hline 15 & 1 & 0 & 2.155206 & 1.244309 & -0.149812 \\
\hline 16 & 1 & 0 & 0.000000 & 2.488618 & 0.149812 \\
\hline 17 & 1 & 0 & -2.155206 & -1.244309 & 0.149812 \\
\hline 18 & 1 & 0 & 2.155206 & -1.244309 & 0.149812 \\
\hline
\end{tabular}

FREQUENCIES AND ROTATIONAL CONSTANTS

INDEX NO. DOF TYPE CM-1(UNSCALED) CM-1(SCALED BY ) SYMMETRY IR-INTENSITY

\begin{tabular}{|c|c|c|c|c|c|}
\hline 1 & vib & 228.33 & 0.00 & EU & 0.0035 \\
\hline 2 & vib & 228.33 & 0.00 & EU & 0.0035 \\
\hline 3 & vib & 375.92 & 0.00 & $\mathrm{~A} 1 \mathrm{G}$ & 0.0000 \\
\hline 4 & vib & 428.88 & 0.00 & EG & 0.0000 \\
\hline 5 & vib & 428.88 & 0.00 & EG & 0.0000 \\
\hline 6 & vib & 522.87 & 0.00 & $\mathrm{~A} 2 \mathrm{U}$ & 0.7189 \\
\hline 7 & vib & 793.51 & 0.00 & EG & 0.0000 \\
\hline 8 & vib & 793.51 & 0.00 & $E G$ & 0.0000 \\
\hline 9 & vib & 800.83 & 0.00 & $\mathrm{~A} 1 \mathrm{G}$ & 0.0000 \\
\hline 10 & vib & 862.84 & 0.00 & $\mathrm{EU}$ & 1.8497 \\
\hline 11 & vib & 862.84 & 0.00 & EU & 1.8500 \\
\hline 12 & vib & 919.02 & 0.00 & EU & 1.8052 \\
\hline 13 & vib & 919.02 & 0.00 & EU & 1.8052 \\
\hline
\end{tabular}




\begin{tabular}{|c|c|c|c|c|c|}
\hline 14 & vib & 1035.92 & 0.00 & EG & 0.0000 \\
\hline 15 & vib & 1035.92 & 0.00 & EG & 0.0000 \\
\hline 16 & vib & 1045.31 & 0.00 & $\mathrm{~A} 2 \mathrm{U}$ & 2.1928 \\
\hline 17 & vib & 1074.77 & 0.00 & A2G & 0.0000 \\
\hline 18 & vib & 1094.00 & 0.00 & $\mathrm{~A} 1 \mathrm{U}$ & 0.0000 \\
\hline 19 & vib & 1130.57 & 0.00 & $\mathrm{~A} 1 \mathrm{U}$ & 0.0000 \\
\hline 20 & vib & 1185.15 & 0.00 & $\mathrm{~A} 1 \mathrm{G}$ & 0.0000 \\
\hline 21 & vib & 1288.18 & 0.00 & $\mathrm{EU}$ & 1.8754 \\
\hline 22 & vib & 1288.18 & 0.00 & $\mathrm{EU}$ & 1.8755 \\
\hline 23 & vib & 1293.05 & 0.00 & EG & 0.0000 \\
\hline 24 & vib & 1293.05 & 0.00 & EG & 0.0000 \\
\hline 25 & vib & 1356.09 & 0.00 & $\mathrm{~A} 2 \mathrm{G}$ & 0.0000 \\
\hline 26 & vib & 1370.56 & 0.00 & $\mathrm{~A} 1 \mathrm{U}$ & 0.0000 \\
\hline 27 & vib & 1378.08 & 0.00 & EG & 0.0000 \\
\hline 28 & vib & 1378.08 & 0.00 & EG & 0.0000 \\
\hline 29 & vib & 1389.06 & 0.00 & $\mathrm{EU}$ & 0.3002 \\
\hline 30 & vib & 1389.06 & 0.00 & $\mathrm{EU}$ & 0.3002 \\
\hline 31 & vib & 1486.63 & 0.00 & EG & 0.0000 \\
\hline 32 & vib & 1486.63 & 0.00 & EG & 0.0000 \\
\hline 33 & vib & 1491.18 & 0.00 & $\mathrm{EU}$ & 4.9491 \\
\hline 34 & vib & 1491.18 & 0.00 & $\mathrm{EU}$ & 4.9493 \\
\hline 35 & vib & 1497.43 & 0.00 & $\mathrm{~A} 2 \mathrm{U}$ & 13.2973 \\
\hline 36 & vib & 1511.65 & 0.00 & $\mathrm{~A} 1 \mathrm{G}$ & 0.0000 \\
\hline 37 & vib & 2995.60 & 0.00 & EU & 24.5150 \\
\hline 38 & vib & 2995.60 & 0.00 & $\mathrm{EU}$ & 24.5150 \\
\hline 39 & vib & 2996.22 & 0.00 & A1G & 0.0000 \\
\hline 40 & vib & 3003.88 & 0.00 & EG & 0.0000 \\
\hline 41 & vib & 3003.88 & 0.00 & EG & 0.0000 \\
\hline 42 & vib & 3007.26 & 0.00 & $\mathrm{~A} 2 \mathrm{U}$ & 74.3058 \\
\hline 43 & vib & 3045.12 & 0.00 & $\mathrm{EU}$ & 123.5855 \\
\hline 44 & vib & 3045.12 & 0.00 & $\mathrm{EU}$ & 123.5842 \\
\hline 45 & vib & 3046.76 & 0.00 & EG & 0.0000 \\
\hline 46 & vib & 3046.76 & 0.00 & EG & 0.0000 \\
\hline 47 & vib & 3049.16 & 0.00 & $\mathrm{~A} 1 \mathrm{G}$ & 0.0000 \\
\hline 48 & vib & 3054.47 & 0.00 & $\mathrm{~A} 2 \mathrm{U}$ & 148.1852 \\
\hline 49 & rot & 0.1436578 & - & & \\
\hline 50 & rot & 0.1436578 & - & & \\
\hline 51 & rot & 0.0818787 & - & & \\
\hline
\end{tabular}

\section{Cyclohexane-chair-z-matrix.log}

Charge $=0$ Multiplicity $=1$ Stoichiometry $\quad$ C6H12

\begin{tabular}{cccccc} 
Center & Atomic & Atomic & \multicolumn{3}{c}{ Coordinates (Angstroms) } \\
Number & Number & Type & X & Y & Z \\
-1 & 6 & 0 & -1.267364 & 0.731713 & 0.227369 \\
2 & 6 & 0 & -0.000000 & -1.463426 & 0.227369 \\
3 & 6 & 0 & 1.267364 & 0.731713 & 0.227369
\end{tabular}




\begin{tabular}{|c|c|c|c|c|c|}
\hline 4 & 6 & 0 & -0.000000 & 1.463426 & -0.227369 \\
\hline 5 & 6 & 0 & -1.267364 & -0.731713 & -0.227369 \\
\hline 6 & 6 & 0 & 1.267364 & -0.731713 & -0.227369 \\
\hline 7 & 1 & 0 & -1.329595 & 0.767642 & 1.320441 \\
\hline 8 & 1 & 0 & -2.155298 & 1.244362 & -0.149730 \\
\hline 9 & 1 & 0 & -0.000000 & -1.535284 & 1.320441 \\
\hline 10 & 1 & 0 & -0.000000 & -2.488724 & -0.149730 \\
\hline 11 & 1 & 0 & 1.329595 & 0.767642 & 1.320441 \\
\hline 12 & 1 & 0 & 2.155298 & 1.244362 & -0.149730 \\
\hline 13 & 1 & 0 & -0.000000 & 1.535284 & -1.320441 \\
\hline 14 & 1 & 0 & -0.000000 & 2.488724 & 0.149730 \\
\hline 15 & 1 & 0 & -1.329595 & -0.767642 & -1.320441 \\
\hline 16 & 1 & 0 & -2.155298 & -1.244362 & 0.149730 \\
\hline 17 & 1 & 0 & 1.329595 & -0.767642 & -1.320441 \\
\hline 18 & 1 & 0 & 2.155298 & -1.244362 & 0.149730 \\
\hline
\end{tabular}

FREQUENCIES AND ROTATIONAL CONSTANTS

$\begin{array}{cccccc}\text { INDEX NO. } & \text { DOF TYPE } & \text { CM-1 (UNSCALED) } & \text { CM-1 (SCALED BY }) & \text { SYMMETRY IR-INTENSITY } \\ 1 & \text { vib } & 228.30 & 0.00 & \text { EU } & 0.0034 \\ 2 & \text { vib } & 228.31 & 0.00 & \text { EU } & 0.0035 \\ 3 & \text { vib } & 375.87 & 0.00 & \text { A1G } & 0.0000 \\ 4 & \text { vib } & 428.82 & 0.00 & \text { EG } & 0.0000 \\ 5 & \text { vib } & 428.83 & 0.00 & \text { EG } & 0.0000 \\ 6 & \text { vib } & 522.78 & 0.00 & \text { A2U } & 0.7204 \\ 7 & \text { vib } & 793.45 & 0.00 & \text { EG } & 0.0000 \\ 8 & \text { vib } & 793.47 & 0.00 & \text { EG } & 0.0000 \\ 9 & \text { vib } & 800.72 & 0.00 & \text { A1G } & 0.0000 \\ 10 & \text { vib } & 862.76 & 0.00 & \text { EU } & 1.8495 \\ 11 & \text { vib } & 862.76 & 0.00 & \text { EU } & 1.8485 \\ 12 & \text { vib } & 918.97 & 0.00 & \text { EU } & 1.8036 \\ 13 & \text { vib } & 918.97 & 0.00 & \text { EU } & 1.8045 \\ 14 & \text { vib } & 1035.84 & 0.00 & \text { EG } & 0.0000 \\ 15 & \text { vib } & 1035.84 & 0.00 & \text { EG } & 0.0000 \\ 16 & \text { vib } & 1045.27 & 0.00 & \text { A2U } & 2.1958 \\ 17 & \text { vib } & 1074.75 & 0.00 & \text { A2G } & 0.0000 \\ 18 & \text { vib } & 1093.93 & 0.00 & \text { A1U } & 0.0000 \\ 19 & \text { vib } & 1130.53 & 0.00 & \text { A1U } & 0.0000 \\ 20 & \text { vib } & 1185.12 & 0.00 & \text { A1G } & 0.0000 \\ 21 & \text { vib } & 1288.17 & 0.00 & \text { EU } & 1.8729 \\ 22 & \text { vib } & 1288.17 & 0.00 & \text { EU } & 1.8712 \\ 23 & \text { vib } & 1293.01 & 0.00 & \text { EG } & 0.0000 \\ 24 & \text { vib } & 1293.01 & 0.00 & \text { EG } & 0.0000 \\ 25 & \text { vib } & 1356.04 & 0.00 & \text { A2G } & 0.0000 \\ 26 & \text { vib } & 1370.52 & 0.00 & \text { A1U } & 0.0000 \\ 27 & \text { vib } & 1378.06 & 0.00 & \text { EG } & 0.0000 \\ 28 & \text { vib } & 1378.06 & 0.00 & \text { EG } & 0.0000 \\ 29 & \text { vib } & 1389.01 & 0.00 & \text { EU } & 0.3019 \\ 30 & \text { vib } & 1389.02 & 0.00 & \text { EU } & 0.3015 \\ 31 & \text { vib } & 1486.63 & 0.00 & 0.00 & 0.0000 \\ 32 & \text { vib } & 1486.63 & 0.00 & 4.9559 \\ 33 & \text { vib } & 1491.16 & 0.00 & 4.9484 \\ 34 & \text { vib } & 1491.17 & & \end{array}$




$\begin{array}{rrrrrr}35 & \text { vib } & 1497.45 & 0.00 & \text { A2U } & 13.2990 \\ 36 & \text { vib } & 1511.63 & 0.00 & \text { A1G } & 0.0000 \\ 37 & \text { vib } & 2995.53 & 0.00 & \text { EU } & 24.4246 \\ 38 & \text { vib } & 2995.54 & 0.00 & \text { EU } & 24.4326 \\ 39 & \text { vib } & 2996.16 & 0.00 & \text { A1G } & 0.0000 \\ 40 & \text { vib } & 3003.86 & 0.00 & \text { EG } & 0.0000 \\ 41 & \text { vib } & 3003.86 & 0.00 & \text { EG } & 0.0000 \\ 42 & \text { vib } & 3007.29 & 0.00 & \text { A2U } & 74.7189 \\ 43 & \text { vib } & 3045.19 & 0.00 & \text { EU } & 123.5979 \\ 44 & \text { vib } & 3045.20 & 0.00 & \text { EU } & 123.6229 \\ 45 & \text { vib } & 3046.79 & 0.00 & \text { EG } & 0.0000 \\ 46 & \text { vib } & 3046.79 & 0.00 & \text { EG } & 0.0000 \\ 47 & \text { vib } & 3049.24 & 0.00 & \text { A1G } & 0.0000 \\ 48 & \text { vib } & 3054.47 & 0.00 & \text { A2U } & 147.9275 \\ 49 & \text { rot } & 0.1436496 & - & & \\ 50 & \text { rot } & 0.1436496 & - & & \\ 51 & \text { rot } & 0.0818717 & - & & \end{array}$

\section{Cyclohexane-D3d-freqs.log}

Charge $=0$ Multiplicity $=1$ Stoichiometry C6H12

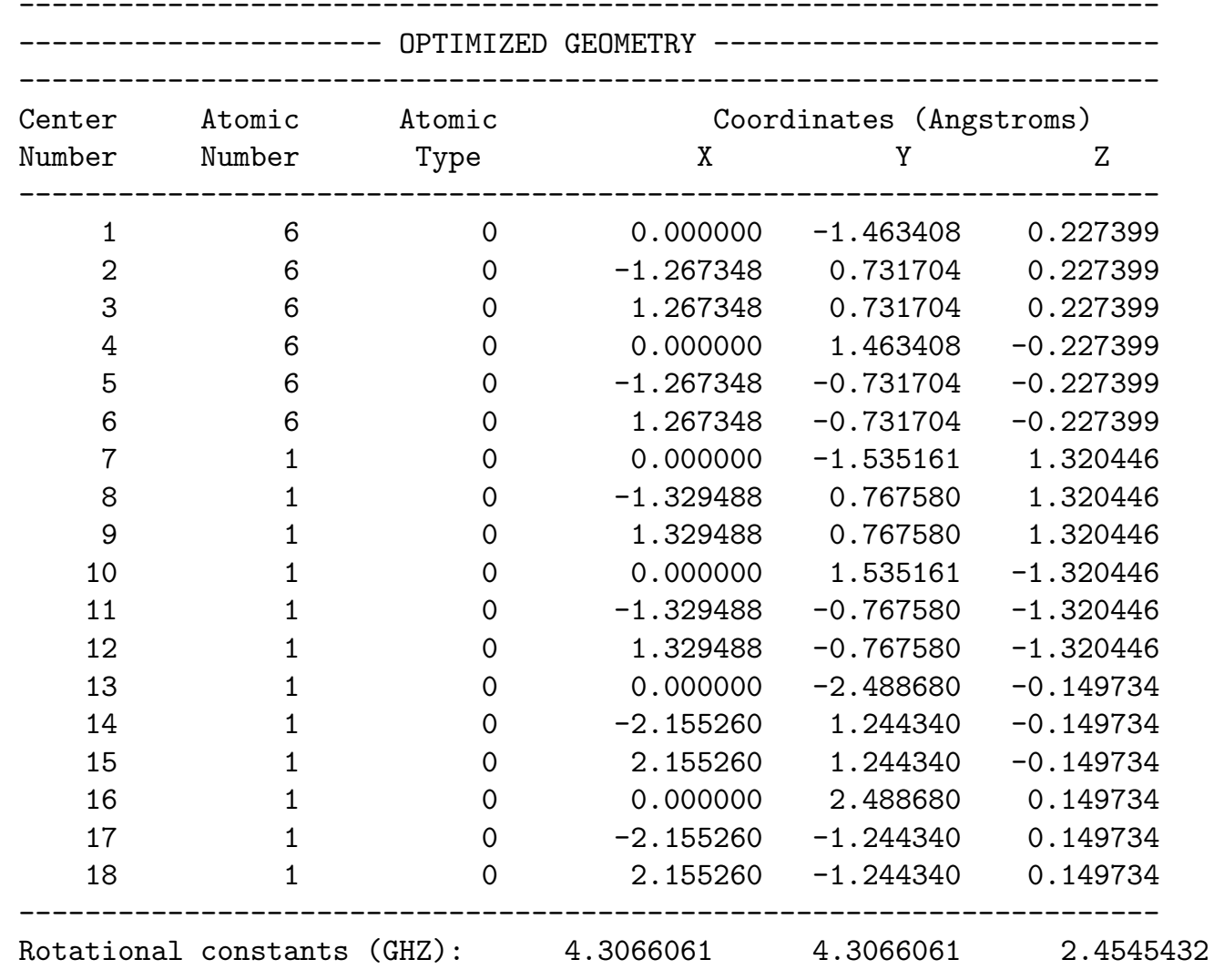




\begin{tabular}{|c|c|c|c|c|c|}
\hline INDEX NO. & DOF TYPE & CM-1 (UNSCALED) & CM-1 (SCALED BY 1) & SYMMETRY & IR-INTENSITY \\
\hline 1 & vib & 228.23 & 228.23 & EU & 0.0035 \\
\hline 2 & vib & 228.23 & 228.23 & EU & 0.0035 \\
\hline 3 & vib & 375.83 & 375.83 & $\mathrm{~A} 1 \mathrm{G}$ & 0.0000 \\
\hline 4 & vib & 428.82 & 428.82 & EG & 0.0000 \\
\hline 5 & vib & 428.82 & 428.82 & EG & 0.0000 \\
\hline 6 & vib & 522.73 & 522.73 & $\mathrm{~A} 2 \mathrm{U}$ & 0.7190 \\
\hline 7 & vib & 793.41 & 793.41 & EG & 0.0000 \\
\hline 8 & vib & 793.41 & 793.41 & EG & 0.0000 \\
\hline 9 & vib & 800.73 & 800.73 & $\mathrm{~A} 1 \mathrm{G}$ & 0.0000 \\
\hline 10 & vib & 862.76 & 862.76 & EU & 1.8511 \\
\hline 11 & vib & 862.76 & 862.76 & EU & 1.8511 \\
\hline 12 & vib & 918.93 & 918.93 & EU & 1.8035 \\
\hline 13 & vib & 918.93 & 918.93 & $\mathrm{EU}$ & 1.8035 \\
\hline 14 & vib & 1035.84 & 1035.84 & EG & 0.0000 \\
\hline 15 & vib & 1035.84 & 1035.84 & EG & 0.0000 \\
\hline 16 & vib & 1045.27 & 1045.27 & $\mathrm{~A} 2 \mathrm{U}$ & 2.1941 \\
\hline 17 & vib & 1074.70 & 1074.70 & A2G & 0.0000 \\
\hline 18 & vib & 1093.92 & 1093.92 & $\mathrm{~A} 1 \mathrm{U}$ & 0.0000 \\
\hline 19 & vib & 1130.49 & 1130.49 & $\mathrm{~A} 1 \mathrm{U}$ & 0.0000 \\
\hline 20 & vib & 1185.12 & 1185.12 & $\mathrm{~A} 1 \mathrm{G}$ & 0.0000 \\
\hline 21 & vib & 1288.15 & 1288.15 & $\mathrm{EU}$ & 1.8728 \\
\hline 22 & vib & 1288.15 & 1288.15 & EU & 1.8728 \\
\hline 23 & vib & 1293.00 & 1293.00 & EG & 0.0000 \\
\hline 24 & vib & 1293.00 & 1293.00 & EG & 0.0000 \\
\hline 25 & vib & 1356.01 & 1356.01 & A2G & 0.0000 \\
\hline 26 & vib & 1370.46 & 1370.46 & $\mathrm{~A} 1 \mathrm{U}$ & 0.0000 \\
\hline 27 & vib & 1377.99 & 1377.99 & EG & 0.0000 \\
\hline 28 & vib & 1377.99 & 1377.99 & EG & 0.0000 \\
\hline 29 & vib & 1388.98 & 1388.98 & EU & 0.3002 \\
\hline 30 & vib & 1388.98 & 1388.98 & EU & 0.3002 \\
\hline 31 & vib & 1486.59 & 1486.59 & EG & 0.0000 \\
\hline 32 & vib & 1486.59 & 1486.59 & EG & 0.0000 \\
\hline 33 & vib & 1491.13 & 1491.13 & EU & 4.9459 \\
\hline 34 & vib & 1491.13 & 1491.13 & EU & 4.9462 \\
\hline 35 & vib & 1497.41 & 1497.41 & $\mathrm{~A} 2 \mathrm{U}$ & 13.3103 \\
\hline 36 & vib & 1511.60 & 1511.60 & $\mathrm{~A} 1 \mathrm{G}$ & 0.0000 \\
\hline 37 & vib & 2995.85 & 2995.85 & EU & 24.5778 \\
\hline 38 & vib & 2995.85 & 2995.85 & EU & 24.5778 \\
\hline 39 & vib & 2996.47 & 2996.47 & $\mathrm{~A} 1 \mathrm{G}$ & 0.0000 \\
\hline 40 & vib & 3004.10 & 3004.10 & EG & 0.0000 \\
\hline 41 & vib & 3004.10 & 3004.10 & EG & 0.0000 \\
\hline 42 & vib & 3007.47 & 3007.47 & $\mathrm{~A} 2 \mathrm{U}$ & 74.1636 \\
\hline 43 & vib & 3045.30 & 3045.30 & $\mathrm{EU}$ & 123.4688 \\
\hline 44 & vib & 3045.30 & 3045.30 & EU & 123.4681 \\
\hline 45 & vib & 3046.96 & 3046.96 & EG & 0.0000 \\
\hline 46 & vib & 3046.96 & 3046.96 & EG & 0.0000 \\
\hline 47 & vib & 3049.34 & 3049.34 & $\mathrm{~A} 1 \mathrm{G}$ & 0.0000 \\
\hline 48 & vib & 3054.69 & 3054.69 & $\mathrm{~A} 2 \mathrm{U}$ & 148.3400 \\
\hline 49 & rot & 0.1436529 & - & & \\
\hline 50 & rot & 0.1436529 & - & & \\
\hline 51 & rot & 0.0818747 & - & & \\
\hline
\end{tabular}




\section{Cyclohexane-D3d.log}

\section{Cyclohexane-twist-boat.log}

Charge $=0$ Multiplicity $=1$ Stoichiometry $\quad$ C6H12

\begin{tabular}{|c|c|c|c|c|c|}
\hline \multirow{2}{*}{$\begin{array}{l}\text { Center } \\
\text { Number }\end{array}$} & \multirow{2}{*}{$\begin{array}{l}\text { Atomic } \\
\text { Number }\end{array}$} & \multirow{2}{*}{$\begin{array}{c}\text { Atomic } \\
\text { Type }\end{array}$} & \multicolumn{3}{|c|}{ Coordinates (Angstroms) } \\
\hline & & & $\mathrm{X}$ & $\mathrm{Y}$ & Z \\
\hline 1 & 6 & 0 & -1.225049 & -0.664614 & 0.379546 \\
\hline 2 & 6 & 0 & -0.000590 & 1.523771 & 0.000337 \\
\hline 3 & 6 & 0 & 1.225049 & -0.663618 & -0.379872 \\
\hline 4 & 6 & 0 & 1.225049 & 0.664614 & 0.379546 \\
\hline 5 & 6 & 0 & 0.000590 & -1.523771 & 0.000337 \\
\hline 6 & 6 & 0 & -1.225049 & 0.663618 & -0.379872 \\
\hline 7 & 1 & 0 & -1.215247 & -0.457259 & 1.453334 \\
\hline 8 & 1 & 0 & -0.254720 & 2.178910 & 0.836636 \\
\hline 9 & 1 & 0 & 2.145814 & -1.217620 & -0.188775 \\
\hline 10 & 1 & 0 & 1.215247 & 0.457259 & 1.453334 \\
\hline 11 & 1 & 0 & 0.254720 & -2.178910 & 0.836636 \\
\hline 12 & 1 & 0 & -2.145814 & 1.217620 & -0.188775 \\
\hline 13 & 1 & 0 & -2.145278 & -1.219133 & 0.187467 \\
\hline 14 & 1 & 0 & 0.252614 & 2.180405 & -0.835067 \\
\hline 15 & 1 & 0 & 1.214281 & -0.456130 & -1.453665 \\
\hline 16 & 1 & 0 & 2.145278 & 1.219133 & 0.187467 \\
\hline 17 & 1 & 0 & -0.252614 & -2.180405 & -0.835067 \\
\hline 18 & 1 & 0 & -1.214281 & 0.456130 & -1.453665 \\
\hline
\end{tabular}

FREQUENCIES AND ROTATIONAL CONSTANTS

INDEX NO. DOF TYPE CM-1(UNSCALED) CM-1 (SCALED BY 1) SYMMETRY IR-INTENSITY

\begin{tabular}{|c|c|c|c|c|c|}
\hline 1 & vib & 100.68 & 100.68 & $\mathrm{~A}$ & 0.0463 \\
\hline 2 & vib & 240.59 & 240.59 & B & 0.0146 \\
\hline 3 & vib & 274.64 & 274.64 & $\mathrm{~A}$ & 0.0000 \\
\hline 4 & vib & 431.78 & 431.78 & A & 0.3309 \\
\hline 5 & vib & 467.74 & 467.74 & $\mathrm{~A}$ & 0.0000 \\
\hline 6 & vib & 561.34 & 561.34 & B & 0.3909 \\
\hline 7 & vib & 770.14 & 770.14 & B & 1.2584 \\
\hline 8 & vib & 780.45 & 780.45 & $\mathrm{~A}$ & 2.1843 \\
\hline 9 & vib & 803.16 & 803.16 & A & 0.0000 \\
\hline 10 & vib & 857.53 & 857.53 & B & 1.2182 \\
\hline 11 & vib & 868.13 & 868.13 & B & 2.0442 \\
\hline 12 & vib & 904.65 & 904.65 & A & 0.0020 \\
\hline 13 & vib & 943.59 & 943.59 & A & 0.0000 \\
\hline 14 & vib & 1019.59 & 1019.59 & B & 1.0733 \\
\hline 15 & vib & 1026.46 & 1026.46 & $\mathrm{~A}$ & 0.0000 \\
\hline 16 & vib & 1054.33 & 1054.33 & A & 0.2810 \\
\hline 17 & vib & 1093.40 & 1093.40 & B & 0.0000 \\
\hline
\end{tabular}




\begin{tabular}{|c|c|c|c|c|c|}
\hline 18 & vib & 1114.74 & 1114.74 & B & 0.0015 \\
\hline 19 & vib & 1153.47 & 1153.47 & B & 0.1056 \\
\hline 20 & vib & 1176.32 & 1176.32 & B & 1.8212 \\
\hline 21 & vib & 1260.06 & 1260.06 & A & 0.3313 \\
\hline 22 & vib & 1270.21 & 1270.21 & A & 0.0000 \\
\hline 23 & vib & 1298.11 & 1298.11 & A & 0.0000 \\
\hline 24 & vib & 1312.82 & 1312.82 & B & 1.6281 \\
\hline 25 & vib & 1346.40 & 1346.40 & A & 0.3257 \\
\hline 26 & vib & 1356.36 & 1356.36 & A & 0.0367 \\
\hline 27 & vib & 1371.90 & 1371.90 & B & 0.6205 \\
\hline 28 & vib & 1378.88 & 1378.88 & A & 0.0000 \\
\hline 29 & vib & 1383.89 & 1383.89 & B & 0.9401 \\
\hline 30 & vib & 1384.38 & 1384.38 & B & 0.3548 \\
\hline 31 & vib & 1492.30 & 1492.30 & A & 0.0000 \\
\hline 32 & vib & 1493.27 & 1493.27 & B & 0.1644 \\
\hline 33 & vib & 1500.89 & 1500.89 & B & 3.6171 \\
\hline 34 & vib & 1505.47 & 1505.47 & B & 4.3213 \\
\hline 35 & vib & 1509.70 & 1509.70 & A & 9.3439 \\
\hline 36 & vib & 1523.39 & 1523.39 & A & 0.0000 \\
\hline 37 & vib & 3002.34 & 3002.34 & B & 17.9749 \\
\hline 38 & vib & 3013.49 & 3013.49 & A & 0.0238 \\
\hline 39 & vib & 3014.23 & 3014.23 & B & 9.5997 \\
\hline 40 & vib & 3019.95 & 3019.95 & A & 51.6914 \\
\hline 41 & vib & 3023.96 & 3023.96 & A & 0.0184 \\
\hline 42 & vib & 3025.43 & 3025.43 & B & 65.9251 \\
\hline 43 & vib & 3041.04 & 3041.04 & A & 18.0757 \\
\hline 44 & vib & 3044.99 & 3044.99 & B & 46.2931 \\
\hline 45 & vib & 3054.78 & 3054.78 & B & 72.4282 \\
\hline 46 & vib & 3060.33 & 3060.33 & A & 0.0091 \\
\hline 47 & vib & 3064.37 & 3064.37 & B & 79.0185 \\
\hline 48 & vib & 3070.80 & 3070.80 & A & 124.5317 \\
\hline 49 & rot & 0.1463361 & - & & \\
\hline 50 & rot & 0.1390098 & - & & \\
\hline 51 & rot & 0.0844256 & - & & \\
\hline
\end{tabular}

\section{Cyclopropenyl-symm.log}

Charge $=0$ Multiplicity $=2$ Stoichiometry C3H3(2)

\begin{tabular}{|c|c|c|c|c|c|}
\hline \multirow{2}{*}{$\begin{array}{l}\text { Center } \\
\text { Number }\end{array}$} & \multirow{2}{*}{$\begin{array}{l}\text { Atomic } \\
\text { Number }\end{array}$} & \multirow{2}{*}{$\begin{array}{c}\text { Atomic } \\
\text { Type }\end{array}$} & \multicolumn{3}{|c|}{ Coordinates (Angstroms) } \\
\hline & & & $\mathrm{X}$ & $\mathrm{Y}$ & Z \\
\hline 1 & 6 & 0 & -0.035930 & 0.884572 & 0.000000 \\
\hline 2 & 6 & 0 & -0.035930 & -0.416848 & 0.653754 \\
\hline 3 & 6 & 0 & -0.035930 & -0.416848 & -0.653754 \\
\hline 4 & 1 & 0 & -0.041921 & -0.981382 & 1.568621 \\
\hline 5 & 1 & 0 & -0.041921 & -0.981382 & -1.568621 \\
\hline 6 & 1 & 0 & 0.730580 & 1.657511 & 0.000000 \\
\hline
\end{tabular}


Rotational constants (GHZ): $\quad 32.1867819 \quad 26.8175454 \quad 15.0509586$

FREQUENCIES AND ROTATIONAL CONSTANTS

\begin{tabular}{|c|c|c|c|c|c|}
\hline INDEX & NO. DOF TYPE & CM-1 (UNSCALED) & CM-1 (SCALED BY ) & SYMMETRY & IR-INTENSITY \\
\hline 1 & vib & 612.64 & 0.00 & $A^{\prime}$ & 64.7522 \\
\hline 2 & vib & 691.71 & 0.00 & A" & 36.4266 \\
\hline 3 & vib & 886.69 & 0.00 & A" & 0.0140 \\
\hline 4 & vib & 917.57 & 0.00 & $A^{\prime}$ & 4.2927 \\
\hline 5 & vib & 979.26 & 0.00 & $A^{\prime}$ & 22.0226 \\
\hline 6 & vib & 982.48 & 0.00 & $A^{\prime \prime}$ & 7.8558 \\
\hline 7 & vib & 1039.54 & 0.00 & $A^{\prime \prime}$ & 22.3607 \\
\hline 8 & vib & 1235.69 & 0.00 & $A^{\prime}$ & 7.7809 \\
\hline 9 & vib & 1662.75 & 0.00 & $A^{\prime}$ & 0.3771 \\
\hline 10 & vib & 3055.64 & 0.00 & $A^{\prime}$ & 56.0600 \\
\hline 11 & vib & 3238.86 & 0.00 & $A^{\prime \prime}$ & 1.8157 \\
\hline 12 & vib & 3284.58 & 0.00 & $A^{\prime}$ & 0.1156 \\
\hline 13 & rot & 1.0736355 & - & & \\
\hline 14 & rot & 0.8945370 & - & & \\
\hline 15 & rot & 0.5020459 & - & & \\
\hline
\end{tabular}

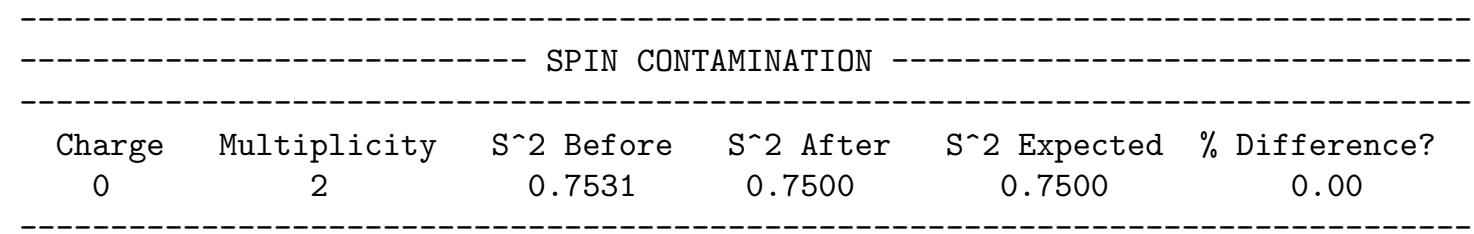

\section{Cyclopropenyl.log}

Charge $=0$ Multiplicity $=2$ Stoichiometry C3H3(2)

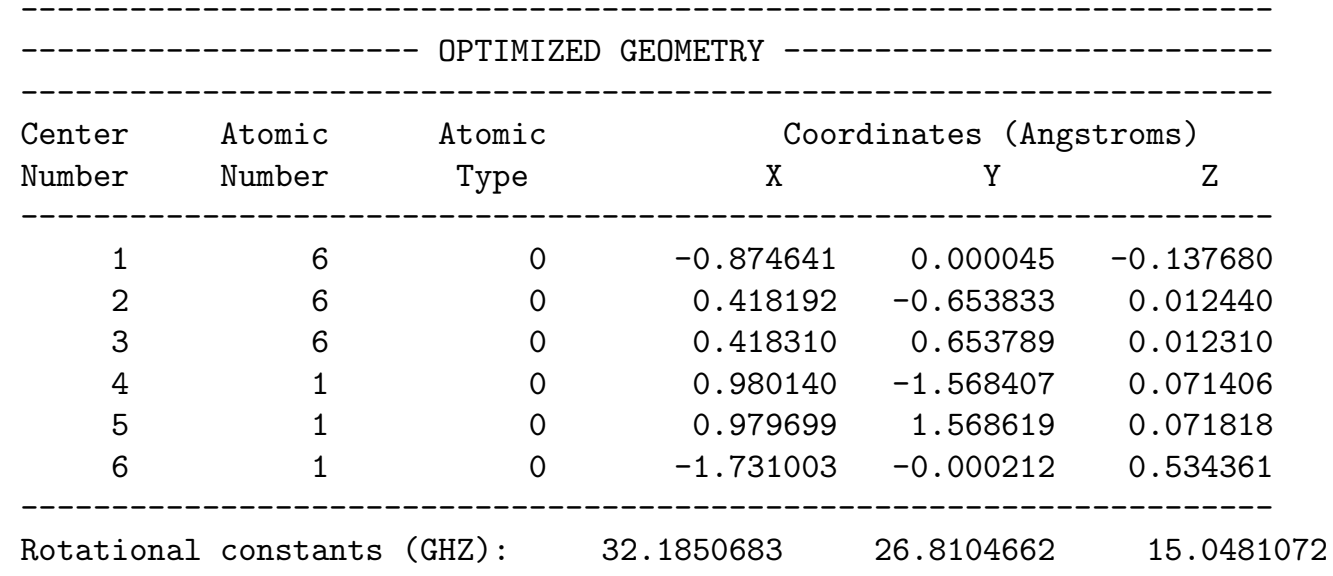

FREQUENCIES AND ROTATIONAL CONSTANTS

INDEX NO. DOF TYPE CM-1(UNSCALED) CM-1(SCALED BY) SYMMETRY IR-INTENSITY 


\begin{tabular}{|c|c|c|c|c|c|}
\hline 1 & vib & 612.29 & 0.00 & $\mathrm{~A}$ & 64.7681 \\
\hline 2 & vib & 691.42 & 0.00 & A & 36.3843 \\
\hline 3 & vib & 886.62 & 0.00 & A & 0.0132 \\
\hline 4 & vib & 917.43 & 0.00 & A & 4.2201 \\
\hline 5 & vib & 978.20 & 0.00 & $\mathrm{~A}$ & 22.0139 \\
\hline 6 & vib & 983.17 & 0.00 & $\mathrm{~A}$ & 7.7994 \\
\hline 7 & vib & 1039.57 & 0.00 & $\mathrm{~A}$ & 22.4713 \\
\hline 8 & vib & 1235.17 & 0.00 & A & 7.8046 \\
\hline 9 & vib & 1662.24 & 0.00 & $\mathrm{~A}$ & 0.3772 \\
\hline 10 & vib & 3055.51 & 0.00 & $\mathrm{~A}$ & 55.9767 \\
\hline 11 & vib & 3239.40 & 0.00 & A & 1.8199 \\
\hline 12 & vib & 3285.07 & 0.00 & A & 0.1167 \\
\hline 13 & rot & 1.0735783 & - & & \\
\hline 14 & rot & 0.8943009 & - & & \\
\hline 15 & rot & 0.5019508 & - & & \\
\hline
\end{tabular}

SPIN CONTAMINATION

$\begin{array}{cccccc}\text { Charge } & \text { Multiplicity } & S^{\wedge} 2 \text { Before } & S^{\wedge} 2 \text { After } & S^{\wedge} 2 \text { Expected } & \% \text { Difference? } \\ 0 & 2 & 0.7531 & 0.7500 & 0.7500 & 0.00\end{array}$

\section{Diazene-cis.log}

Charge $=0$ Multiplicity $=1$ Stoichiometry H2N2

\begin{tabular}{|c|c|c|c|c|c|}
\hline \multirow{2}{*}{$\begin{array}{l}\text { Center } \\
\text { Number }\end{array}$} & \multirow{2}{*}{$\begin{array}{l}\text { Atomic } \\
\text { Number }\end{array}$} & \multirow{2}{*}{$\begin{array}{c}\text { Atomic } \\
\text { Type }\end{array}$} & \multicolumn{3}{|c|}{ Coordinates (Angstroms) } \\
\hline & & & $\mathrm{X}$ & Y & $\mathrm{Z}$ \\
\hline 1 & 7 & 0 & 0.000000 & 0.616948 & -0.119635 \\
\hline 2 & 7 & 0 & 0.000000 & -0.616948 & -0.119635 \\
\hline 3 & 1 & 0 & 0.000000 & 1.021270 & 0.837447 \\
\hline 4 & 1 & 0 & 0.000000 & -1.021270 & 0.837447 \\
\hline
\end{tabular}

\section{FREQUENCIES AND ROTATIONAL CONSTANTS}

$\begin{array}{ccccrr}\text { INDEX NO. DOF TYPE } & \text { CM-1 (UNSCALED) } & \text { CM-1(SCALED BY } & \text { 1) } & \text { SYMMETRY } & \text { IR-INTENSITY } \\ 1 & \text { vib } & 1284.04 & 1284.04 & \mathrm{~A} 2 & 0.0000 \\ 2 & \text { vib } & 1366.83 & 1366.83 & \mathrm{~A} 1 & 4.0375 \\ 3 & \text { vib } & 1567.07 & 1567.07 & \mathrm{~B} 2 & 71.2687 \\ 4 & \text { vib } & 1654.65 & 1654.65 & \mathrm{~A} 1 & 5.8993 \\ 5 & \text { vib } & 3077.03 & 3077.03 & \mathrm{~B} 2 & 93.0868 \\ 6 & \text { vib } & 3176.39 & 3176.39 & \mathrm{~A} 1 & 47.2941 \\ 7 & \text { rot } & 9.7873697 & - & & \\ 8 & \text { rot } & 1.3209084 & - & & \end{array}$




\section{Diazene-trans.log}

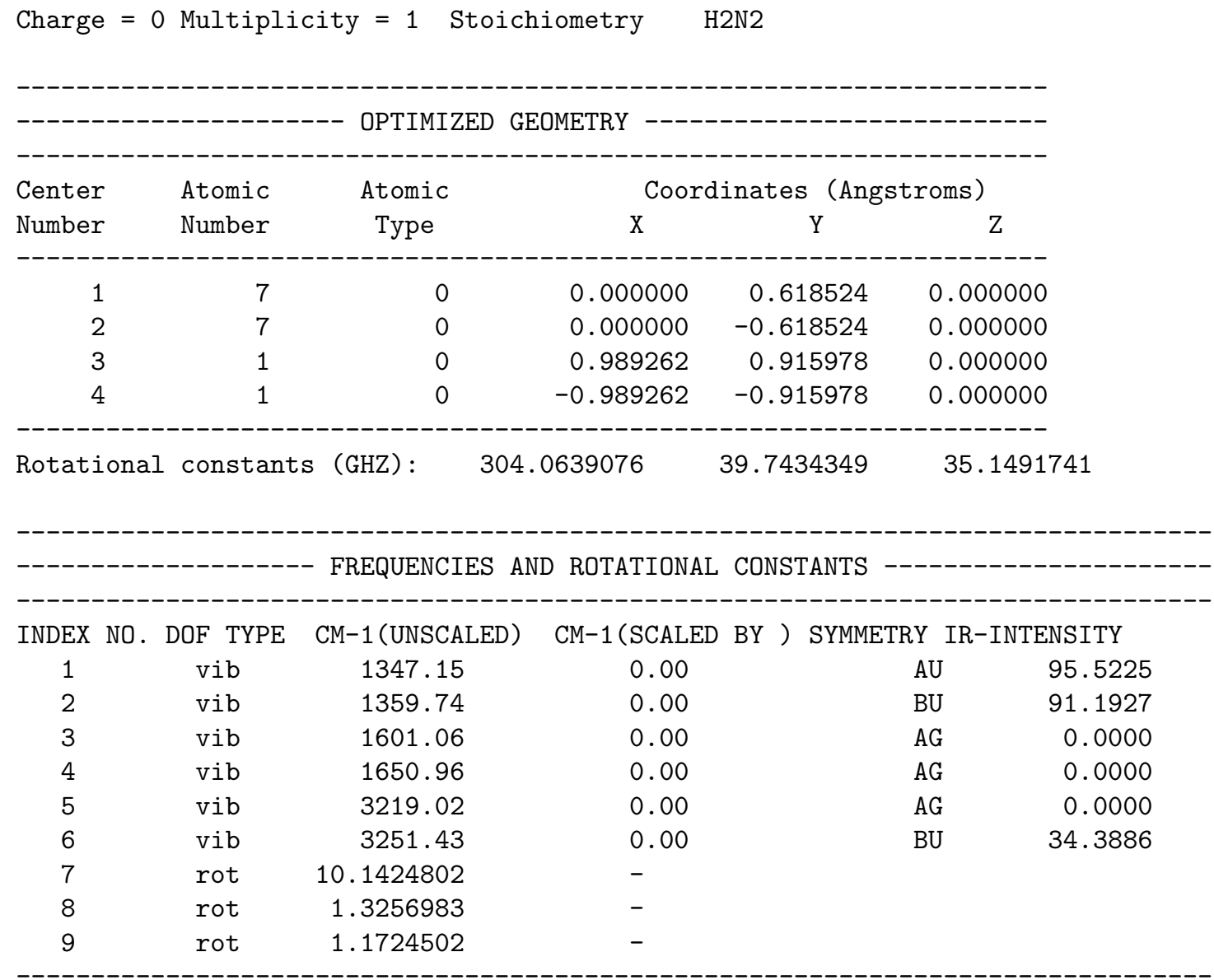

\section{Diazenyl.log}

Charge $=0$ Multiplicity $=2$ Stoichiometry HN2(2)

\begin{tabular}{|c|c|c|c|c|c|}
\hline \multirow{2}{*}{$\begin{array}{l}\text { Center } \\
\text { Number }\end{array}$} & \multirow{2}{*}{$\begin{array}{l}\text { Atomic } \\
\text { Number }\end{array}$} & \multirow{2}{*}{$\begin{array}{c}\text { Atomic } \\
\text { Type }\end{array}$} & \multicolumn{3}{|c|}{ Coordinates (Angstroms) } \\
\hline & & & $\mathrm{X}$ & Y & $\mathrm{Z}$ \\
\hline 1 & 7 & 0 & -0.062532 & -0.512627 & 0.000000 \\
\hline 2 & 7 & 0 & -0.062532 & 0.655623 & 0.000000 \\
\hline 3 & 1 & 0 & 0.875444 & -1.000976 & 0.000000 \\
\hline
\end{tabular}


FREQUENCIES AND ROTATIONAL CONSTANTS

$\begin{array}{cccccc}\text { INDEX NO. DOF TYPE } & \text { CM-1(UNSCALED) } & \text { CM-1(SCALED BY ) } & \text { SYMMETRY } & \text { IR-INTENSITY } \\ 1 & \text { vib } & 1124.65 & 0.00 & \text { A', } & 96.0343 \\ 2 & \text { vib } & 1876.57 & 0.00 & \text { A' } & 23.8858 \\ 3 & \text { vib } & 2777.28 & 0.00 & \text { A' } & 71.0232 \\ 4 & \text { rot } & 22.2015699 & - & & \\ 5 & \text { rot } & 1.5650539 & - & \\ 6 & \text { rot } & 1.4619937 & - & \end{array}$

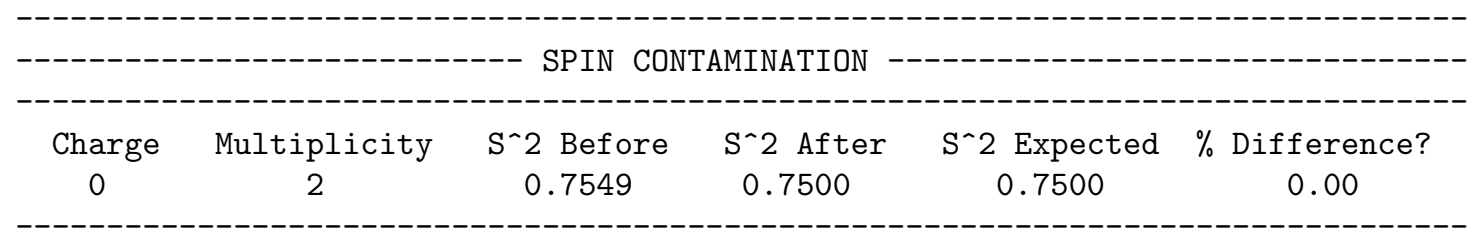

\section{Diisocyanogen-mp2.log}

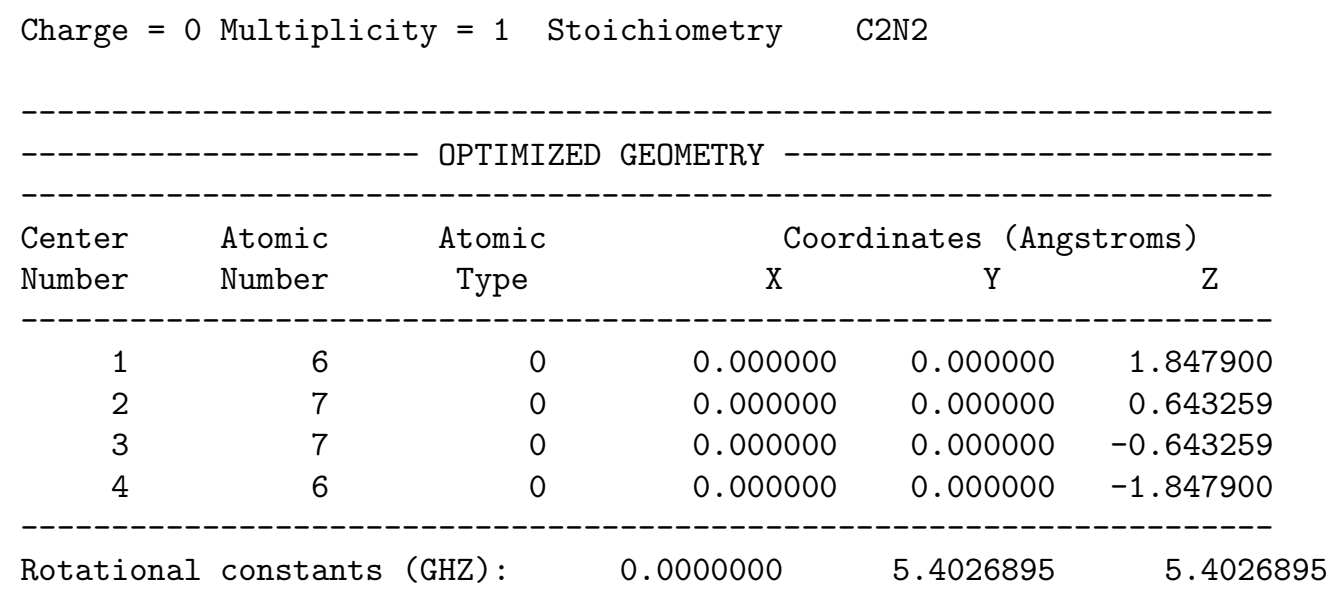

\section{FREQUENCIES AND ROTATIONAL CONSTANTS}

$\begin{array}{ccccrr}\text { INDEX NO. DOF TYPE } & \text { CM-1 (UNSCALED) } & \text { CM-1(SCALED BY } & \text { 1) } & \text { SYMMETRY } & \text { IR-INTENSITY } \\ 1 & \text { vib } & 180.78 & 180.78 & \text { PIU } & 5.8551 \\ 2 & \text { vib } & 180.78 & 180.78 & \text { PIU } & 5.8551 \\ 3 & \text { vib } & 316.42 & 316.42 & \text { PIG } & 0.0000 \\ 4 & \text { vib } & 316.42 & 316.42 & \text { PIG } & 0.0000 \\ 5 & \text { vib } & 941.55 & 941.55 & \text { SGG } & 0.0000 \\ 6 & \text { vib } & 1946.25 & 1946.25 & \text { SGU } & 165.5386 \\ 7 & \text { vib } & 2199.95 & 2199.95 & \text { SGG } & 0.0000 \\ 8 & \text { vib } & 0.00 & 0.00 & & \\ 9 & \text { vib } & 0.00 & 0.00 & & \\ 10 & \text { rot } & 0.0000000 & - & & \\ 11 & \text { rot } & 0.1802143 & - & & \end{array}$




\section{Diisocyanogen.log}

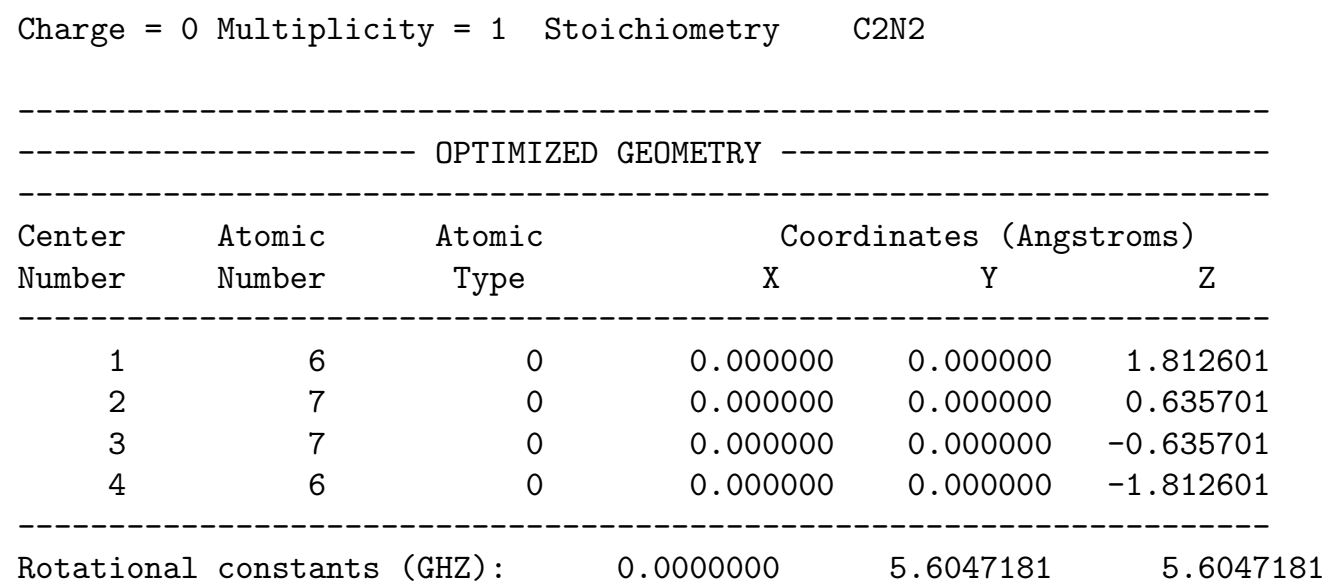

FREQUENCIES AND ROTATIONAL CONSTANTS

$\begin{array}{cccccr}\text { INDEX NO. } & \text { DOF TYPE } & \text { CM-1 (UNSCALED) } & \text { CM-1 (SCALED BY }) & \text { SYMMETRY IR-INTENSITY } \\ 1 & \text { vib } & 204.11 & 0.00 & \text { PI } & 5.4226 \\ 2 & \text { vib } & 204.11 & 0.00 & \text { PI } & 5.4226 \\ 3 & \text { vib } & 366.42 & 0.00 & \text { PI } & 0.0000 \\ 4 & \text { vib } & 366.42 & 0.00 & \text { PI } & 0.0000 \\ 5 & \text { vib } & 977.02 & 0.00 & \text { SG } & 0.0000 \\ 6 & \text { vib } & 2076.54 & 0.00 & \text { SG } & 273.7684 \\ 7 & \text { vib } & 2288.84 & 0.00 & \text { SG } & 0.0000 \\ 8 & \text { vib } & 0.00 & 0.00 & & \\ 9 & \text { vib } & 0.00 & 0.00 & & \\ 10 & \text { rot } & 0.0000000 & - & & \\ 11 & \text { rot } & 0.1869533 & - & & \\ 12 & \text { rot } & 0.1869533 & - & & \end{array}$

\section{Dioxirane.log}

Charge $=0$ Multiplicity $=1$ Stoichiometry $\quad$ CH2O2

\begin{tabular}{|c|c|c|c|c|c|}
\hline \multirow{2}{*}{$\begin{array}{l}\text { Center } \\
\text { Number }\end{array}$} & \multirow{2}{*}{$\begin{array}{l}\text { Atomic } \\
\text { Number }\end{array}$} & \multirow{2}{*}{$\begin{array}{c}\text { Atomic } \\
\text { Type }\end{array}$} & \multicolumn{3}{|c|}{ Coordinates (Angstroms) } \\
\hline & & & $\mathrm{X}$ & $\mathrm{Y}$ & Z \\
\hline 1 & 6 & 0 & 0.000000 & 0.000000 & 0.730486 \\
\hline 2 & 8 & 0 & 0.000000 & 0.749034 & -0.436831 \\
\hline 3 & 8 & 0 & 0.000000 & -0.749034 & -0.436831 \\
\hline 4 & 1 & 0 & 0.925561 & 0.000000 & 1.303190 \\
\hline 5 & 1 & 0 & -0.925561 & 0.000000 & 1.303190 \\
\hline
\end{tabular}




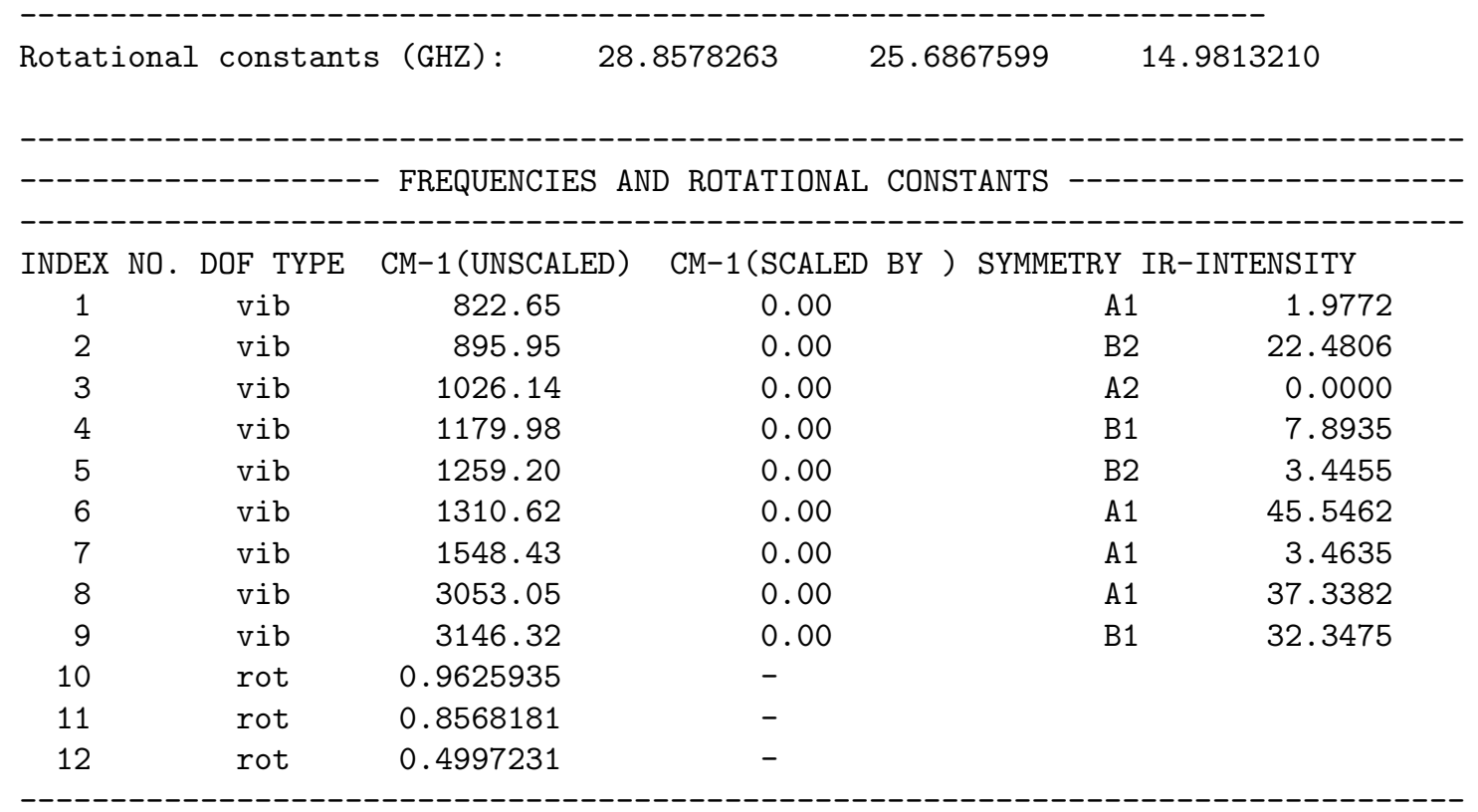

\section{Ethoxy-freqs-vibrot.log}

Charge $=0$ Multiplicity $=2$ Stoichiometry $\quad$ C2H5O(2)

\begin{tabular}{|c|c|c|c|c|c|}
\hline \multirow{2}{*}{$\begin{array}{l}\text { Center } \\
\text { Number }\end{array}$} & \multirow{2}{*}{$\begin{array}{l}\text { Atomic } \\
\text { Number }\end{array}$} & \multirow{2}{*}{$\begin{array}{c}\text { Atomic } \\
\text { Type }\end{array}$} & \multicolumn{3}{|c|}{ Coordinates (Angstroms) } \\
\hline & & & $\mathrm{X}$ & $\mathrm{Y}$ & $\mathrm{Z}$ \\
\hline 1 & 6 & 0 & 1.041700 & -0.602284 & 0.000000 \\
\hline 2 & 6 & 0 & 0.000000 & 0.508116 & 0.000000 \\
\hline 3 & 8 & 0 & -1.302200 & 0.104516 & 0.000000 \\
\hline 4 & 1 & 0 & 2.051400 & -0.187184 & 0.000000 \\
\hline 5 & 1 & 0 & 0.928400 & -1.231184 & 0.883100 \\
\hline 6 & 1 & 0 & 0.928400 & -1.231184 & -0.883100 \\
\hline 7 & 1 & 0 & 0.129600 & 1.189216 & 0.862900 \\
\hline 8 & 1 & 0 & 0.129600 & 1.189216 & -0.862900 \\
\hline
\end{tabular}

FREQUENCIES AND ROTATIONAL CONSTANTS

INDEX NO. DOF TYPE CM-1(UNSCALED) CM-1(SCALED BY) SYMMETRY IR-INTENSITY

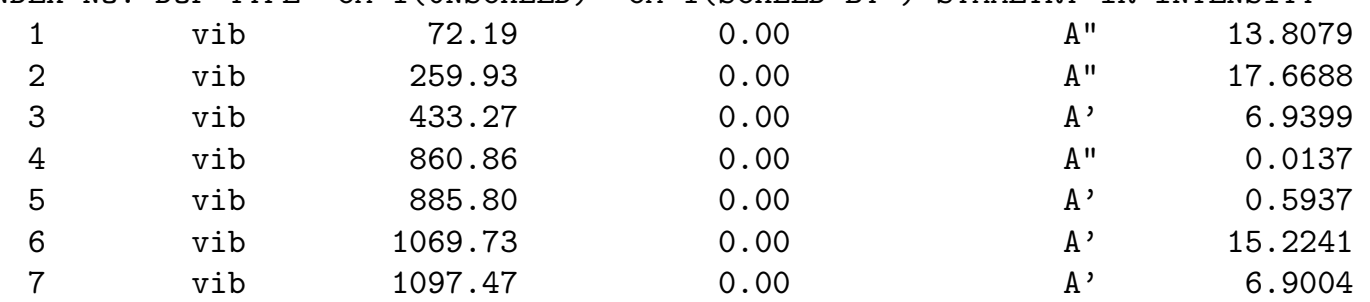




\begin{tabular}{|c|c|c|c|c|c|}
\hline 8 & vib & 1238.80 & 0.00 & $A^{\prime \prime}$ & 0.0123 \\
\hline 9 & vib & 1332.61 & 0.00 & $A^{\prime}$ & 10.9228 \\
\hline 10 & $\mathrm{vib}$ & 1391.20 & 0.00 & $A^{\prime}$ & 23.6838 \\
\hline 11 & vib & 1407.70 & 0.00 & $A^{\prime}$ & 17.7488 \\
\hline 12 & vib & 1485.23 & 0.00 & $A^{\prime \prime}$ & 5.7989 \\
\hline 13 & vib & 1495.70 & 0.00 & $A^{\prime}$ & 5.6207 \\
\hline 14 & vib & 2881.84 & 0.00 & $A^{\prime \prime}$ & 6.0362 \\
\hline 15 & vib & 2882.31 & 0.00 & $A^{\prime}$ & 27.0193 \\
\hline 16 & vib & 3031.90 & 0.00 & $A^{\prime}$ & 13.6034 \\
\hline 17 & vib & 3096.21 & 0.00 & $A^{\prime}$ & 22.5338 \\
\hline 18 & vib & 3105.65 & 0.00 & $A^{\prime \prime}$ & 19.8422 \\
\hline 19 & rot & 1.3354935 & - & & \\
\hline 20 & rot & 0.3188692 & - & & \\
\hline 21 & rot & 0.2840657 & - & & \\
\hline
\end{tabular}

\begin{tabular}{|c|c|c|c|c|c|}
\hline Charge & Multiplicity & $\mathrm{S}^{\wedge} 2$ Before & $S^{\wedge} 2$ After & $S^{\wedge} 2$ Expected & $\%$ Difference? \\
\hline 0 & 2 & 0.7537 & 0.7500 & 0.7500 & 0.00 \\
\hline
\end{tabular}

2) LEVEL OF THEORY: B3LYP/CC-PVTZ+D

Charge $=0$ Multiplicity $=2$ Stoichiometry $\quad$ C2H50(2)

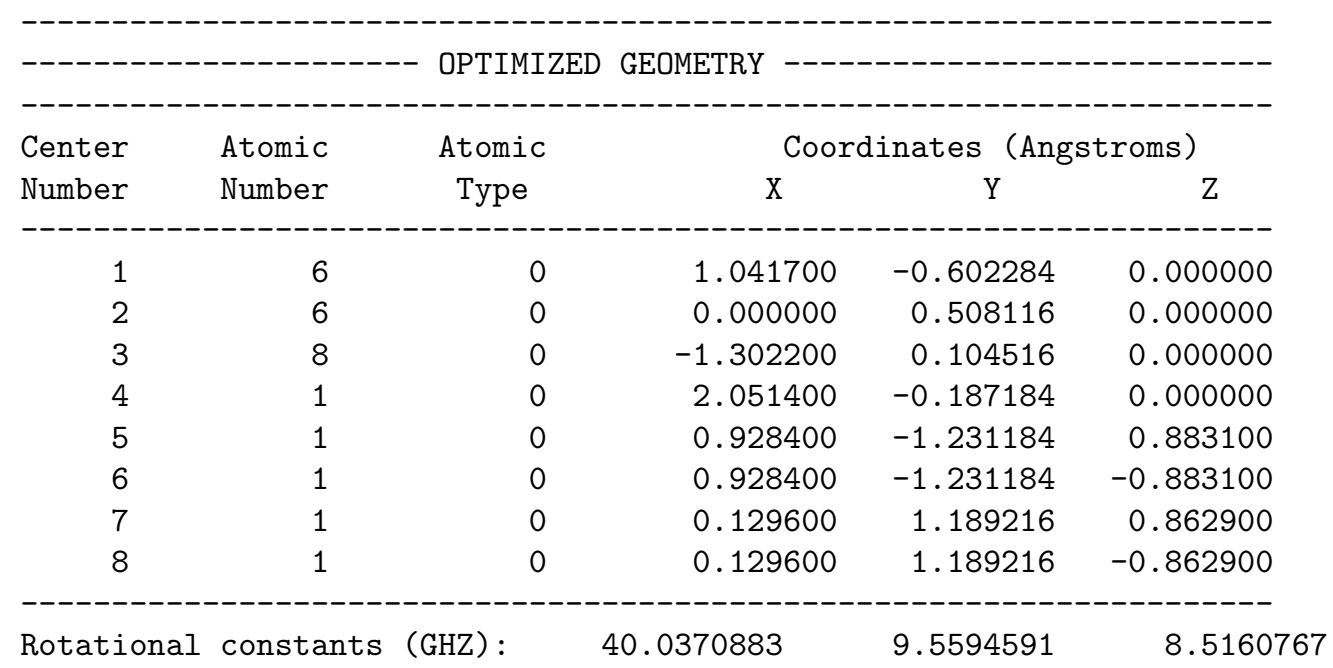

FREQUENCIES AND ROTATIONAL CONSTANTS

$\begin{array}{ccccrr}\text { INDEX NO. DOF TYPE } & \text { CM-1 (UNSCALED) } & \text { CM-1 (SCALED BY ) } & \text { SYMMETRY } & \text { IR-INTENSITY } \\ 1 & \text { vib } & 72.19 & 0.00 & A^{\prime \prime} & 13.8079 \\ 2 & \text { vib } & 259.93 & 0.00 & A^{\prime \prime} & 17.6688 \\ 3 & \text { vib } & 433.27 & 0.00 & A^{\prime} & 6.9399 \\ 4 & \text { vib } & 860.86 & 0.00 & A^{\prime \prime} & 0.0137 \\ 5 & \text { vib } & 885.80 & 0.00 & A^{\prime} & 0.5937 \\ 6 & \text { vib } & 1069.73 & 0.00 & A^{\prime} & 15.2241 \\ 7 & \text { vib } & 1097.47 & 0.00 & A^{\prime} & 6.9004\end{array}$




$\begin{array}{rrrrrr}8 & \text { vib } & 1238.80 & 0.00 & A^{\prime \prime} & 0.0123 \\ 9 & \text { vib } & 1332.61 & 0.00 & A^{\prime} & 10.9228 \\ 10 & \text { vib } & 1391.20 & 0.00 & A^{\prime} & 23.6838 \\ 11 & \text { vib } & 1407.70 & 0.00 & A^{\prime} & 17.7488 \\ 12 & \text { vib } & 1485.23 & 0.00 & A^{\prime \prime} & 5.7989 \\ 13 & \text { vib } & 1495.70 & 0.00 & A^{\prime} & 5.6207 \\ 14 & \text { vib } & 2881.84 & 0.00 & A^{\prime \prime} & 6.0362 \\ 15 & \text { vib } & 2882.31 & 0.00 & A^{\prime} & 27.0193 \\ 16 & \text { vib } & 3031.90 & 0.00 & A^{\prime} & 13.6034 \\ 17 & \text { vib } & 3096.21 & 0.00 & A^{\prime} & 22.5338 \\ 18 & \text { vib } & 3105.65 & 0.00 & & 19.8422 \\ 19 & \text { rot } & 1.3354935 & - & & \\ 20 & \text { rot } & 0.3188692 & - & & \\ 21 & \text { rot } & 0.2840657 & - & & \end{array}$

\section{Ethoxy-freqs.log}

Charge $=0$ Multiplicity $=2$ Stoichiometry $\mathrm{C} 2 \mathrm{H} 50(2)$

\begin{tabular}{|c|c|c|c|c|c|}
\hline \multirow{2}{*}{$\begin{array}{l}\text { Center } \\
\text { Number }\end{array}$} & \multirow{2}{*}{$\begin{array}{l}\text { Atomic } \\
\text { Number }\end{array}$} & \multirow{2}{*}{$\begin{array}{l}\text { Atomic } \\
\text { Type }\end{array}$} & \multicolumn{3}{|c|}{ Coordinates (Angstroms) } \\
\hline & & & $\mathrm{X}$ & Y & $\mathrm{Z}$ \\
\hline 1 & 6 & 0 & 1.041520 & -0.602848 & 0.000000 \\
\hline 2 & 6 & 0 & 0.000000 & 0.508359 & 0.000000 \\
\hline 3 & 8 & 0 & -1.302118 & 0.105033 & 0.000000 \\
\hline 4 & 1 & 0 & 2.051434 & -0.188185 & 0.000000 \\
\hline 5 & 1 & 0 & 0.927693 & -1.231559 & 0.883126 \\
\hline 6 & 1 & 0 & 0.927693 & -1.231559 & -0.883126 \\
\hline 7 & 1 & 0 & 0.130504 & 1.188985 & 0.863068 \\
\hline 8 & 1 & 0 & 0.130504 & 1.188985 & -0.863068 \\
\hline
\end{tabular}

FREQUENCIES AND ROTATIONAL CONSTANTS

$\begin{array}{ccccrr}\text { INDEX NO. DOF TYPE } & \text { CM-1 (UNSCALED) } & \text { CM-1 (SCALED BY } & \text { 1) } & \text { SYMMETRY } & \text { IR-INTENSITY } \\ 1 & \text { vib } & -66.37 & -66.37 & A^{\prime \prime} & 17.5911 \\ 2 & \text { vib } & 255.46 & 255.46 & A^{\prime \prime} & 15.1740 \\ 3 & \text { vib } & 433.94 & 433.94 & A^{\prime} & 6.9187 \\ 4 & \text { vib } & 862.75 & 862.75 & A^{\prime \prime} & 0.0151 \\ 5 & \text { vib } & 886.01 & 886.01 & A^{\prime} & 0.6188\end{array}$




\begin{tabular}{|c|c|c|c|c|c|}
\hline 6 & vib & 1069.92 & 1069.92 & $A^{\prime}$ & 15.6833 \\
\hline 7 & vib & 1097.76 & 1097.76 & $A^{\prime}$ & 6.4884 \\
\hline 8 & vib & 1240.47 & 1240.47 & $\mathrm{~A}^{\prime \prime}$ & 0.0142 \\
\hline 9 & vib & 1333.01 & 1333.01 & $A^{\prime}$ & 11.1239 \\
\hline 10 & vib & 1393.11 & 1393.11 & $A^{\prime}$ & 23.4670 \\
\hline 11 & vib & 1409.78 & 1409.78 & $A^{\prime}$ & 17.7131 \\
\hline 12 & vib & 1485.91 & 1485.91 & $A^{\prime \prime}$ & 5.7988 \\
\hline 13 & vib & 1496.08 & 1496.08 & $A^{\prime}$ & 5.5946 \\
\hline 14 & vib & 2882.70 & 2882.70 & $A^{\prime \prime}$ & 5.9416 \\
\hline 15 & vib & 2882.90 & 2882.90 & $A^{\prime}$ & 26.9937 \\
\hline 16 & vib & 3031.55 & 3031.55 & $A^{\prime}$ & 13.6844 \\
\hline 17 & vib & 3095.75 & 3095.75 & $A^{\prime}$ & 22.4590 \\
\hline 18 & vib & 3105.79 & 3105.79 & $A^{\prime \prime}$ & 19.8179 \\
\hline 19 & rot & 1.3351568 & - & & \\
\hline 20 & rot & 0.3188566 & - & & \\
\hline 21 & rot & 0.2840469 & - & & \\
\hline
\end{tabular}

SPIN CONTAMINATION

Charge Multiplicity $S^{\wedge} 2$ Before $S^{\wedge} 2$ After $S^{\wedge} 2$ Expected \% Difference?
0
2
0.7537
0.7500
0.7500
0.00

\section{Ethylidene-singlet-loose.log}

Charge = 0 Multiplicity $=1 \quad$ Stoichiometry $\quad \mathrm{C} 2 \mathrm{H} 4$

\begin{tabular}{|c|c|c|c|c|c|}
\hline \multirow{2}{*}{$\begin{array}{l}\text { Center } \\
\text { Number }\end{array}$} & \multirow{2}{*}{$\begin{array}{l}\text { Atomic } \\
\text { Number }\end{array}$} & \multirow{2}{*}{$\begin{array}{c}\text { Atomic } \\
\text { Type }\end{array}$} & \multicolumn{3}{|c|}{ Coordinates (Angstroms) } \\
\hline & & & $\mathrm{X}$ & $\mathrm{Y}$ & $\mathrm{Z}$ \\
\hline 1 & 6 & 0 & -0.845029 & -0.190303 & -0.038773 \\
\hline 2 & 6 & 0 & 0.590417 & 0.022101 & -0.031015 \\
\hline 3 & 1 & 0 & -1.310708 & 0.813276 & 0.004069 \\
\hline 4 & 1 & 0 & 1.202762 & -0.840936 & -0.292912 \\
\hline 5 & 1 & 0 & 1.026744 & 0.964237 & -0.382665 \\
\hline 6 & 1 & 0 & 0.608879 & 0.072636 & 1.090237 \\
\hline
\end{tabular}

$\begin{array}{llll}\text { Rotational constants (GHZ): } \quad 122.7922613 & 26.8586145 & 25.2406488\end{array}$

FREQUENCIES AND ROTATIONAL CONSTANTS

$\begin{array}{ccccrr}\text { INDEX NO. DOF TYPE } & \text { CM-1 (UNSCALED) } & \text { CM-1 (SCALED BY } & \text { 1) } & \text { SYMMETRY } & \text { IR-INTENSITY } \\ 1 & \text { vib } & 459.60 & 459.60 & \mathrm{~A} & 5.8447 \\ 2 & \text { vib } & 619.87 & 619.87 & \mathrm{~A} & 39.0831 \\ 3 & \text { vib } & 957.99 & 957.99 & \mathrm{~A} & 21.7773 \\ 4 & \text { vib } & 1124.35 & 1124.35 & \mathrm{~A} & 3.2748 \\ 5 & \text { vib } & 1261.11 & 1261.11 & \mathrm{~A} & 11.8005\end{array}$




\begin{tabular}{|c|c|c|c|c|c|}
\hline 6 & vib & 1301.58 & 1301.58 & A & 41.4142 \\
\hline 7 & vib & 1345.77 & 1345.77 & A & 9.0318 \\
\hline 8 & vib & 1509.99 & 1509.99 & A & 3.8587 \\
\hline 9 & vib & 2820.58 & 2820.58 & A & 15.6465 \\
\hline 10 & vib & 2916.38 & 2916.38 & A & 87.3808 \\
\hline 11 & vib & 2991.73 & 2991.73 & A & 51.0257 \\
\hline 12 & vib & 3081.07 & 3081.07 & A & 23.6795 \\
\hline 13 & rot & 4.0959090 & - & & \\
\hline 14 & rot & 0.8959069 & - & & \\
\hline 15 & rot & 0.8419374 & - & & \\
\hline
\end{tabular}

\section{Ethylidene-singlet-scan.log}

\section{Ethylidene-singlet.log}

\begin{tabular}{|c|c|c|c|c|c|}
\hline \multirow{2}{*}{$\begin{array}{l}\text { Center } \\
\text { Number }\end{array}$} & \multirow{2}{*}{$\begin{array}{l}\text { Atomic } \\
\text { Number }\end{array}$} & \multirow{2}{*}{$\begin{array}{c}\text { Atomic } \\
\text { Type }\end{array}$} & \multicolumn{3}{|c|}{ Coordinates (Angstroms) } \\
\hline & & & $\mathrm{X}$ & Y & $\mathrm{Z}$ \\
\hline 1 & 6 & 0 & 0.078512 & 0.881506 & 0.000000 \\
\hline 2 & 6 & 0 & 0.078512 & -0.585743 & 0.000000 \\
\hline 3 & 1 & 0 & -0.990747 & 1.172129 & 0.000000 \\
\hline 4 & 1 & 0 & 1.062947 & -1.052143 & 0.000000 \\
\hline 5 & 1 & 0 & -0.507170 & -0.947281 & 0.862245 \\
\hline 6 & 1 & 0 & -0.507170 & -0.947281 & -0.862245 \\
\hline
\end{tabular}

FREQUENCIES AND ROTATIONAL CONSTANTS

INDEX NO. DOF TYPE CM-1(UNSCALED) CM-1(SCALED BY) SYMMETRY IR-INTENSITY

\begin{tabular}{|c|c|c|c|c|c|}
\hline 1 & vib & -413.23 & 0.00 & $A^{\prime \prime}$ & 32.8307 \\
\hline 2 & vib & 814.07 & 0.00 & $A^{\prime \prime}$ & 0.4615 \\
\hline 3 & vib & 902.79 & 0.00 & $A^{\prime}$ & 21.7577 \\
\hline 4 & vib & 1073.06 & 0.00 & $A^{\prime}$ & 5.2874 \\
\hline 5 & vib & 1247.29 & 0.00 & $A^{\prime}$ & 23.0451 \\
\hline 6 & vib & 1296.73 & 0.00 & $A^{\prime}$ & 39.0472 \\
\hline 7 & vib & 1453.08 & 0.00 & $A^{\prime \prime}$ & 12.3851 \\
\hline 8 & vib & 1461.24 & 0.00 & $A^{\prime}$ & 0.7818 \\
\hline 9 & vib & 2901.48 & 0.00 & $A^{\prime}$ & 67.6608 \\
\hline 10 & vib & 2939.03 & 0.00 & $A^{\prime}$ & 19.4039 \\
\hline 11 & vib & 2965.31 & 0.00 & $A^{\prime \prime}$ & 3.3266 \\
\hline 12 & vib & 3080.85 & 0.00 & $A^{\prime}$ & 40.7089 \\
\hline 13 & rot & 4.0333893 & - & & \\
\hline 14 & rot & 0.8770412 & - & & \\
\hline
\end{tabular}




\section{Ethylidene-triplet.log}

\begin{tabular}{|c|c|c|c|c|c|}
\hline \multirow{2}{*}{$\begin{array}{l}\text { Center } \\
\text { Number }\end{array}$} & \multirow{2}{*}{$\begin{array}{l}\text { Atomic } \\
\text { Number }\end{array}$} & \multirow{2}{*}{$\begin{array}{l}\text { Atomic } \\
\text { Type }\end{array}$} & \multicolumn{3}{|c|}{ Coordinates (Angstroms) } \\
\hline & & & $\mathrm{X}$ & Y & Z \\
\hline 1 & 6 & 0 & 0.046999 & 0.850431 & 0.000000 \\
\hline 2 & 6 & 0 & 0.046999 & -0.609899 & 0.000000 \\
\hline 3 & 1 & 0 & -0.720874 & 1.609638 & 0.000000 \\
\hline 4 & 1 & 0 & 1.067383 & -1.002988 & 0.000000 \\
\hline 5 & 1 & 0 & -0.455251 & -1.024922 & 0.884933 \\
\hline 6 & 1 & 0 & -0.455251 & -1.024922 & -0.884933 \\
\hline
\end{tabular}

FREQUENCIES AND ROTATIONAL CONSTANTS

\begin{tabular}{|c|c|c|c|c|c|}
\hline INDEX & NO. DOF TYPE & CM-1 (UNSCALED) & CM-1 (SCALED BY ) & SYMMETRY & IR-INTENSITY \\
\hline 1 & vib & 180.60 & 0.00 & $A^{\prime \prime}$ & 30.7748 \\
\hline 2 & vib & 760.38 & 0.00 & $A^{\prime}$ & 11.7963 \\
\hline 3 & vib & 995.35 & 0.00 & A" & 1.1126 \\
\hline 4 & vib & 1070.40 & 0.00 & $A^{\prime}$ & 1.9338 \\
\hline 5 & vib & 1102.25 & 0.00 & $A^{\prime}$ & 0.1980 \\
\hline 6 & vib & 1388.52 & 0.00 & $A^{\prime}$ & 1.8961 \\
\hline 7 & vib & 1451.11 & 0.00 & $A^{\prime \prime}$ & 6.2257 \\
\hline 8 & vib & 1451.77 & 0.00 & $A^{\prime}$ & 5.9518 \\
\hline 9 & vib & 2944.68 & 0.00 & $A^{\prime}$ & 23.0204 \\
\hline 10 & vib & 2977.56 & 0.00 & $A^{\prime \prime}$ & 22.0973 \\
\hline 11 & vib & 3043.67 & 0.00 & $A^{\prime}$ & 11.7562 \\
\hline 12 & vib & 3210.52 & 0.00 & $A^{\prime}$ & 6.1933 \\
\hline 13 & rot & 4.6516525 & - & & \\
\hline 14 & rot & 0.8232759 & - & & \\
\hline 15 & rot & 0.8049156 & - & & \\
\hline
\end{tabular}

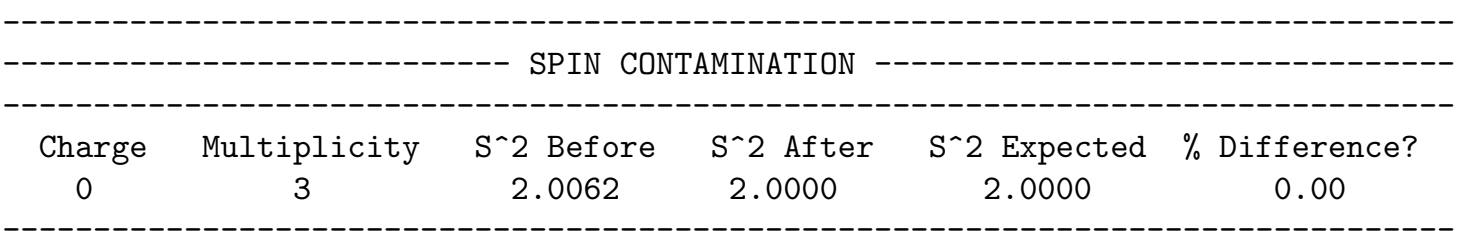




\section{$56 \quad$ Ethylidyne.log}

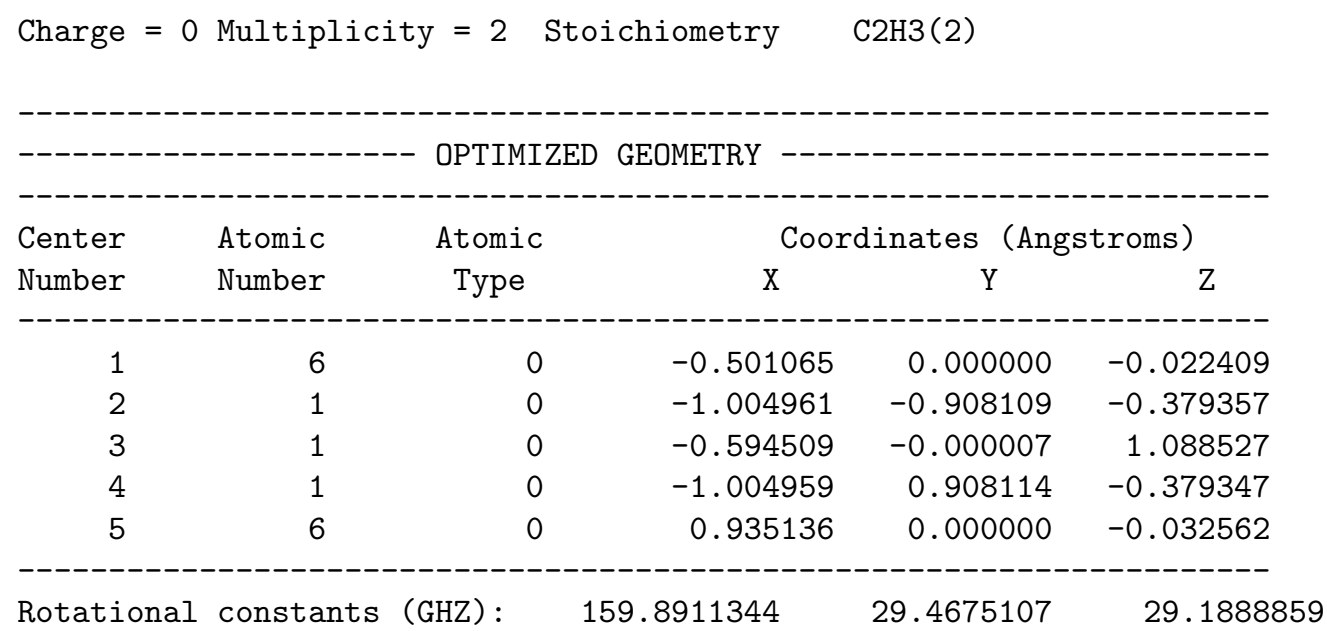

FREQUENCIES AND ROTATIONAL CONSTANTS

INDEX NO. DOF TYPE CM-1(UNSCALED) CM-1 (SCALED BY) SYMMETRY IR-INTENSITY

\begin{tabular}{|c|c|c|c|c|c|}
\hline 1 & vib & 522.47 & 0.00 & A & 4.2564 \\
\hline 2 & vib & 853.89 & 0.00 & $\mathrm{~A}$ & 0.1314 \\
\hline 3 & vib & 1102.35 & 0.00 & $\mathrm{~A}$ & 14.4516 \\
\hline 4 & vib & 1292.09 & 0.00 & $\mathrm{~A}$ & 21.6608 \\
\hline 5 & vib & 1306.20 & 0.00 & $\mathrm{~A}$ & 71.3635 \\
\hline 6 & vib & 1442.25 & 0.00 & A & 7.1231 \\
\hline 7 & vib & 2877.05 & 0.00 & A & 12.0234 \\
\hline 8 & vib & 2963.47 & 0.00 & $\mathrm{~A}$ & 22.2680 \\
\hline 9 & vib & 2994.39 & 0.00 & $\mathrm{~A}$ & 18.6018 \\
\hline 10 & rot & 5.3333942 & - & & \\
\hline 11 & rot & 0.9829304 & - & & \\
\hline 12 & rot & 0.9736364 & - & & \\
\hline
\end{tabular}

\begin{tabular}{|c|c|c|c|c|c|}
\hline Charge & Multiplicity & $\mathrm{S}^{\wedge} 2$ Before & $S^{\wedge} 2$ After & $\mathrm{S}^{\wedge} 2$ Expected & $\%$ Difference? \\
\hline 0 & 2 & 0.7543 & 0.7500 & 0.7500 & 0.00 \\
\hline
\end{tabular}

\section{Ethynol.log}

Charge $=0$ Multiplicity $=1$ Stoichiometry $\quad$ C2H2O

\begin{tabular}{|c|c|c|c|c|c|}
\hline Center & Atomic & Atomic & \multicolumn{3}{|c|}{ Coordinates (Angstroms) } \\
\hline Number & Number & Type & $\mathrm{X}$ & $\mathrm{Y}$ & $\mathrm{Z}$ \\
\hline 1 & 6 & 0 & 0.063967 & 1.318541 & 0.000000 \\
\hline
\end{tabular}

Page 60 of 107 


\begin{tabular}{cccccc}
2 & 6 & 0 & 0.000000 & 0.123395 & 0.000000 \\
3 & 1 & 0 & 0.127981 & 2.375831 & 0.000000 \\
4 & 8 & 0 & -0.152360 & -1.176400 & 0.000000 \\
5 & 1 & 0 & 0.707098 & -1.616247 & 0.000000 \\
\hline Rotational constants (GHZ) : & 678.4580398 & 9.7811466 & 9.6421385
\end{tabular}

FREQUENCIES AND ROTATIONAL CONSTANTS

$\begin{array}{ccccrr}\text { INDEX NO. DOF TYPE } & \text { CM-1 (UNSCALED) } & \text { CM-1 (SCALED BY ) } & \text { SYMMETRY } & \text { IR-INTENSITY } \\ 1 & \text { vib } & 401.58 & 0.00 & A^{\prime} & 11.8590 \\ 2 & \text { vib } & 440.52 & 0.00 & A^{\prime \prime} & 4.4563 \\ 3 & \text { vib } & 545.78 & 0.00 & A^{\prime \prime} & 63.9004 \\ 4 & \text { vib } & 649.98 & 0.00 & A^{\prime} & 49.2427 \\ 5 & \text { vib } & 1084.42 & 0.00 & A^{\prime} & 95.4519 \\ 6 & \text { vib } & 1256.44 & 0.00 & A^{\prime} & 89.9575 \\ 7 & \text { vib } & 2282.74 & 0.00 & A^{\prime} & 142.8150 \\ 8 & \text { vib } & 3489.11 & 0.00 & A^{\prime} & 95.7666 \\ 9 & \text { vib } & 3764.91 & 0.00 & & 105.8902 \\ 10 & \text { rot } & 22.6309242 & - & & \\ 11 & \text { rot } & 0.3262639 & - & - & \end{array}$

\section{Formic-acid-anti.log}

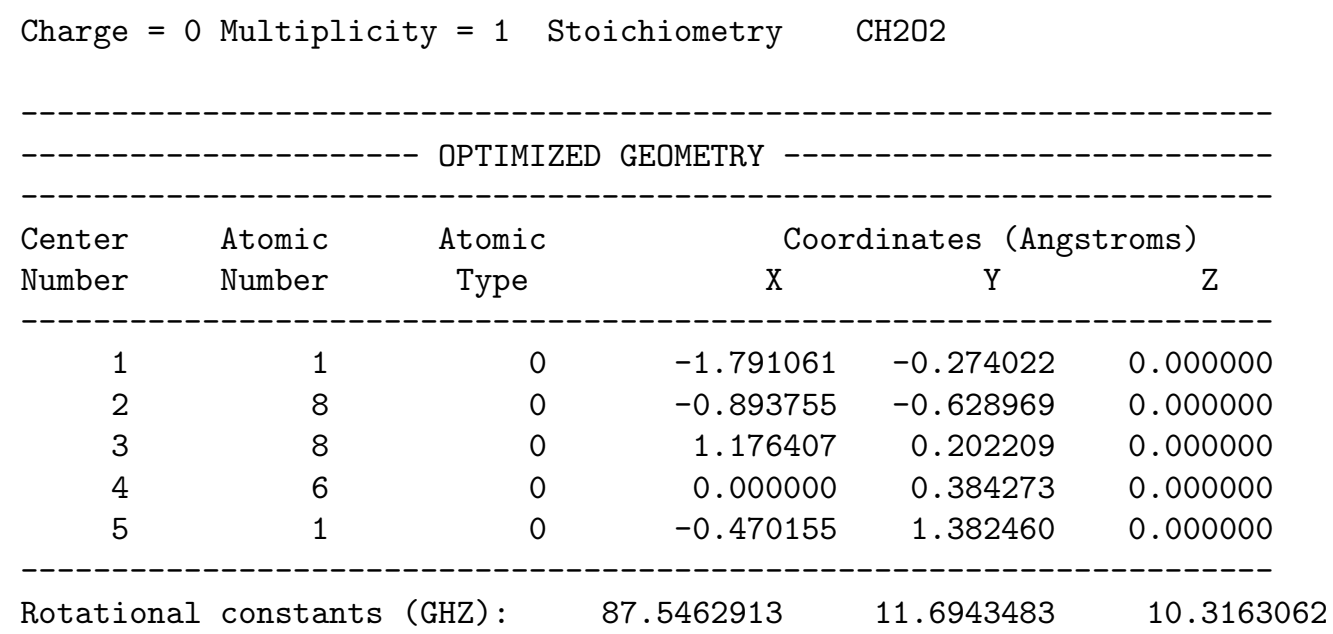

FREQUENCIES AND ROTATIONAL CONSTANTS

$\begin{array}{ccccrr}\text { INDEX NO. DOF TYPE } & \text { CM-1(UNSCALED) } & \text { CM-1 (SCALED BY ) } & \text { SYMMETRY IR-INTENSITY } \\ 1 & \text { vib } & 532.33 & 0.00 & A^{\prime \prime} & 84.2258 \\ 2 & \text { vib } & 661.93 & 0.00 & A^{\prime} & 9.3177 \\ 3 & \text { vib } & 1036.54 & 0.00 & A^{\prime \prime} & 0.0981 \\ 4 & \text { vib } & 1111.25 & 0.00 & A^{\prime} & 49.4539 \\ 5 & \text { vib } & 1274.87 & 0.00 & A^{\prime} & 309.3208 \\ 6 & \text { vib } & 1420.45 & 0.00 & A^{\prime} & 0.4388\end{array}$




\begin{tabular}{rrrllr}
7 & vib & 1871.01 & 0.00 & $A^{\prime}$ & 280.4628 \\
8 & vib & 2952.87 & 0.00 & $A^{\prime}$ & 88.4088 \\
9 & vib & 3788.94 & 0.00 & $A^{\prime}$ & 54.0735 \\
10 & rot & 2.9202299 & - & & \\
11 & rot & 0.3900815 & - & & \\
12 & rot & 0.3441149 & - & & \\
\hline
\end{tabular}

\section{FormicAcidSyn-freqs.log}

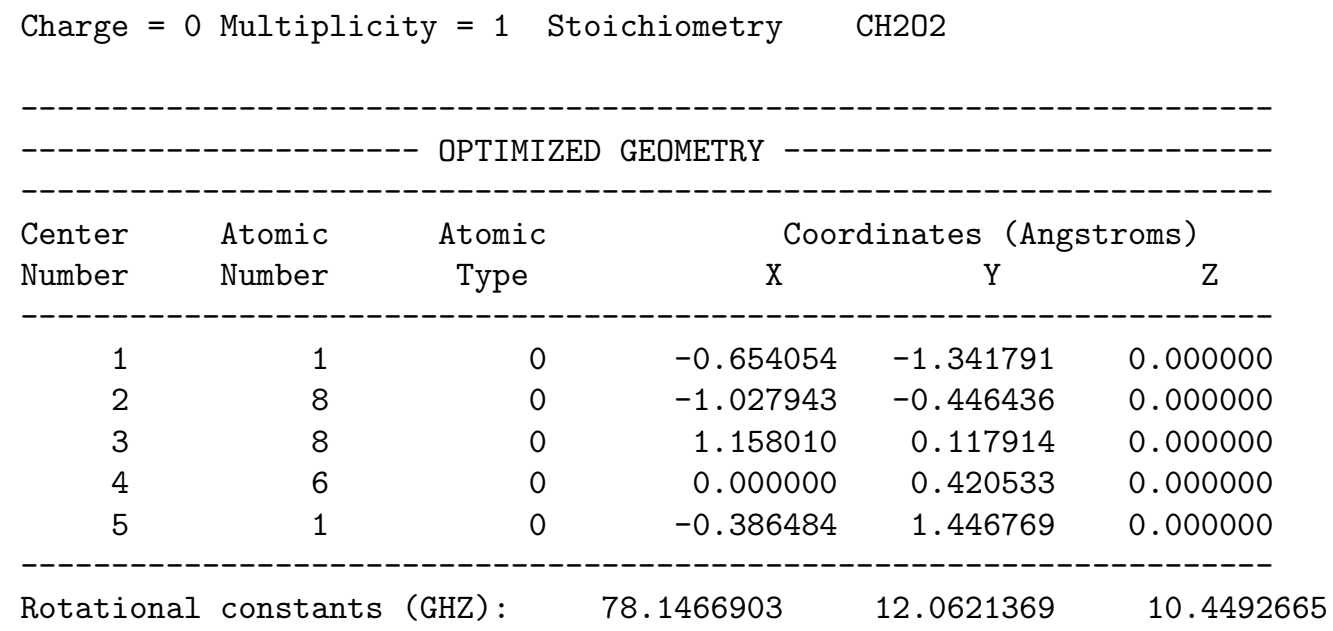

FREQUENCIES AND ROTATIONAL CONSTANTS

INDEX NO. DOF TYPE CM-1(UNSCALED) CM-1(SCALED BY) SYMMETRY IR-INTENSITY

$\begin{array}{rrrrrr}1 & \text { vib } & 630.15 & 0.00 & A^{\prime} & 43.7266 \\ 2 & \text { vib } & 686.62 & 0.00 & A^{\prime \prime} & 138.9135 \\ 3 & \text { vib } & 1055.75 & 0.00 & A^{\prime \prime} & 1.5792 \\ 4 & \text { vib } & 1125.00 & 0.00 & A^{\prime} & 250.5674 \\ 5 & \text { vib } & 1306.18 & 0.00 & A^{\prime} & 8.4558 \\ 6 & \text { vib } & 1405.67 & 0.00 & A^{\prime} & 2.6262 \\ 7 & \text { vib } & 1826.27 & 0.00 & A^{\prime} & 339.6844 \\ 8 & \text { vib } & 3043.76 & 0.00 & A^{\prime} & 47.7048 \\ 9 & \text { vib } & 3722.15 & 0.00 & & 55.0646 \\ 10 & \text { rot } & 2.6066930 & - & & \\ 11 & \text { rot } & 0.4023496 & - & & \\ 12 & \text { rot } & 0.3485500 & - & & \end{array}$

\section{Formyloxy.log}

Charge $=0$ Multiplicity $=2$ Stoichiometry $\quad$ CHO2(2) 


\begin{tabular}{|c|c|c|c|c|c|}
\hline \multirow{2}{*}{$\begin{array}{l}\text { Center } \\
\text { Number }\end{array}$} & \multirow{2}{*}{$\begin{array}{l}\text { Atomic } \\
\text { Number }\end{array}$} & \multirow{2}{*}{$\begin{array}{c}\text { Atomic } \\
\text { Type }\end{array}$} & \multicolumn{3}{|c|}{ Coordinates (Angstroms) } \\
\hline & & & $\mathrm{X}$ & $\mathrm{Y}$ & Z \\
\hline 1 & 8 & 0 & -1.042265 & -0.257926 & 0.000000 \\
\hline 2 & 8 & 0 & 1.042266 & -0.257912 & 0.000000 \\
\hline 3 & 6 & 0 & 0.000000 & 0.432938 & 0.000000 \\
\hline 4 & 1 & 0 & -0.000012 & 1.529080 & 0.000000 \\
\hline
\end{tabular}

\section{FREQUENCIES AND ROTATIONAL CONSTANTS}

INDEX NO. DOF TYPE CM-1(UNSCALED) CM-1(SCALED BY) SYMMETRY IR-INTENSITY

$\begin{array}{rrrrrr}1 & \text { vib } & 652.57 & 0.00 & \text { A', } & 23.1972 \\ 2 & \text { vib } & 1027.24 & 0.00 & \text { A" } & 0.4044 \\ 3 & \text { vib } & 1061.49 & 0.00 & \text { A', } & 203.8736 \\ 4 & \text { vib } & 1289.21 & 0.00 & \text { A', } & 105.8114 \\ 5 & \text { vib } & 1496.17 & 0.00 & \text { A', } & 66.6415 \\ 6 & \text { vib } & 3046.66 & 0.00 & \text { A', } & 46.0711 \\ 7 & \text { rot } & 2.5225873 & - & & \\ 8 & \text { rot } & 0.4850961 & - & & \\ 9 & \text { rot } & 0.4068571 & - & \end{array}$

$\begin{array}{cccccc}\text { Charge } & \text { Multiplicity } & \mathrm{S}^{\wedge} 2 \text { Before } & \mathrm{S}^{\wedge} 2 \text { After } & \mathrm{S}^{\wedge} 2 \text { Expected } & \% \text { Difference? } \\ 0 & 2 & 0.7565 & 0.7500 & 0.7500 & 0.00\end{array}$

\section{FulminicAcid-HCNO.log}

Charge $=0$ Multiplicity $=1$ Stoichiometry CHNO

\begin{tabular}{|c|c|c|c|c|c|}
\hline \multirow{2}{*}{$\begin{array}{l}\text { Center } \\
\text { Number }\end{array}$} & \multirow{2}{*}{$\begin{array}{l}\text { Atomic } \\
\text { Number }\end{array}$} & \multirow{2}{*}{$\begin{array}{l}\text { Atomic } \\
\text { Type }\end{array}$} & \multicolumn{3}{|c|}{ Coordinates (Angstroms) } \\
\hline & & & $\mathrm{X}$ & Y & $\mathrm{Z}$ \\
\hline 1 & 8 & 0 & 0.000000 & 0.000000 & 1.178751 \\
\hline 2 & 7 & 0 & 0.000000 & 0.000000 & -0.019877 \\
\hline 3 & 6 & 0 & 0.000000 & 0.000000 & -1.176001 \\
\hline 4 & 1 & 0 & 0.000000 & 0.000000 & -2.234861 \\
\hline
\end{tabular}




$\begin{array}{rrrrrr}1 & \text { vib } & 204.05 & 0.00 & \text { PI } & 79.0837 \\ 2 & \text { vib } & 204.05 & 0.00 & \text { PI } & 79.0837 \\ 3 & \text { vib } & 568.36 & 0.00 & \text { PI } & 1.4660 \\ 4 & \text { vib } & 568.36 & 0.00 & \text { PI } & 1.4660 \\ 5 & \text { vib } & 1292.78 & 0.00 & \text { SG } & 127.2383 \\ 6 & \text { vib } & 2326.48 & 0.00 & \text { SG } & 389.9435 \\ 7 & \text { vib } & 3504.24 & 0.00 & \text { SG } & 257.3657 \\ 8 & \text { vib } & 0.00 & 0.00 & & \\ 9 & \text { vib } & 0.00 & 0.00 & & \\ 10 & \text { rot } & 0.0000000 & - & & \\ 11 & \text { rot } & 0.3853571 & - & & \\ 12 & \text { rot } & 0.3853571 & - & \end{array}$

\section{Glyoxal-cis.log}

\begin{tabular}{|c|c|c|c|c|c|}
\hline \multirow{2}{*}{$\begin{array}{l}\text { Center } \\
\text { Number }\end{array}$} & \multirow{2}{*}{$\begin{array}{l}\text { Atomic } \\
\text { Number }\end{array}$} & \multirow{2}{*}{$\begin{array}{c}\text { Atomic } \\
\text { Type }\end{array}$} & \multicolumn{3}{|c|}{ Coordinates (Angstroms) } \\
\hline & & & $\mathrm{X}$ & $\mathrm{Y}$ & $\mathrm{z}$ \\
\hline 1 & 8 & 0 & 0.000000 & 1.420666 & -0.536414 \\
\hline 2 & 6 & 0 & 0.000000 & 0.770897 & 0.468950 \\
\hline 3 & 1 & 0 & 0.000000 & 1.233910 & 1.477612 \\
\hline 4 & 6 & 0 & 0.000000 & -0.770897 & 0.468950 \\
\hline 5 & 1 & 0 & 0.000000 & -1.233910 & 1.477612 \\
\hline 6 & 8 & 0 & 0.000000 & -1.420666 & -0.536414 \\
\hline
\end{tabular}

FREQUENCIES AND ROTATIONAL CONSTANTS

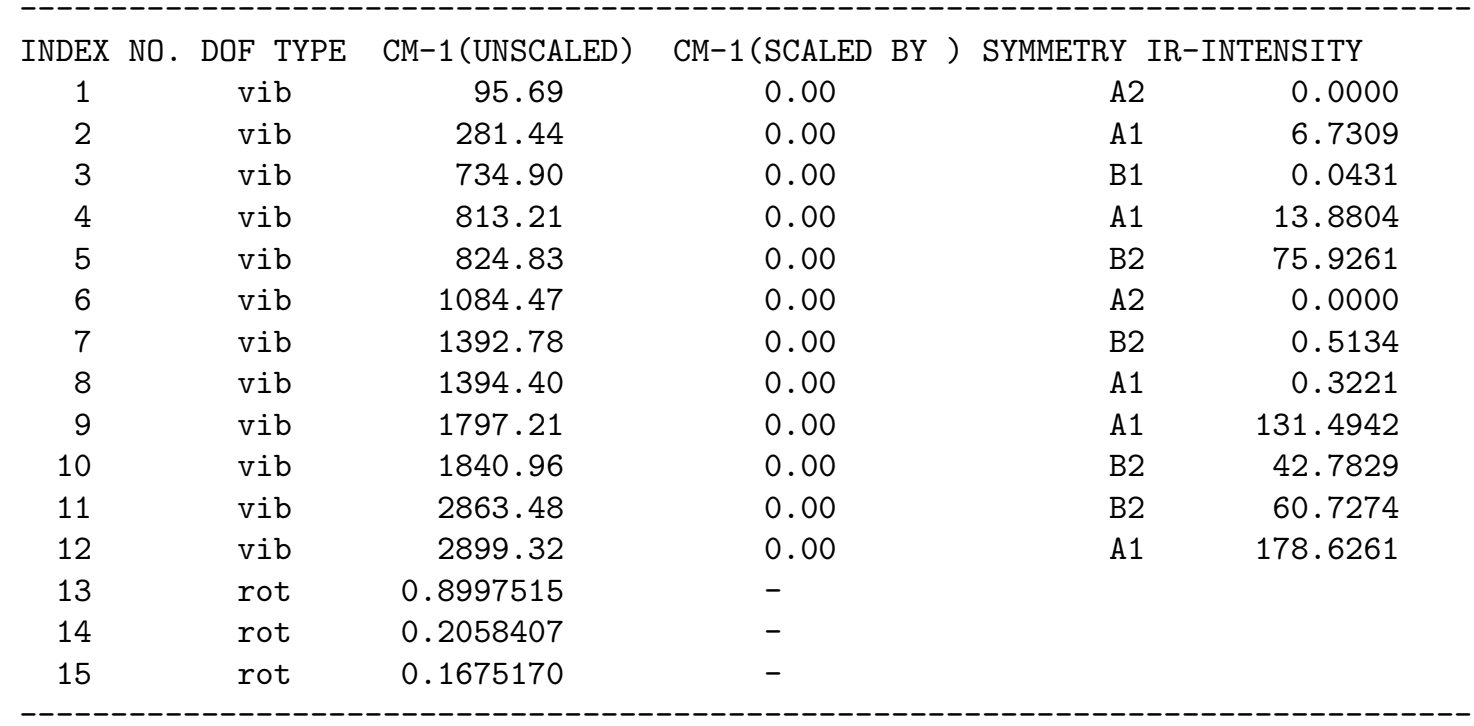




\section{Glyoxal-trans.log}

\begin{tabular}{|c|c|c|c|c|c|}
\hline \multirow{2}{*}{$\begin{array}{l}\text { Center } \\
\text { Number }\end{array}$} & \multirow{2}{*}{$\begin{array}{l}\text { Atomic } \\
\text { Number }\end{array}$} & \multirow{2}{*}{$\begin{array}{c}\text { Atomic } \\
\text { Type }\end{array}$} & \multicolumn{3}{|c|}{ Coordinates (Angstroms) } \\
\hline & & & $\mathrm{X}$ & Y & Z \\
\hline 1 & 8 & 0 & 0.648536 & 1.600954 & 0.000000 \\
\hline 2 & 6 & 0 & 0.648536 & 0.399437 & 0.000000 \\
\hline 3 & 1 & 0 & 1.568648 & -0.214185 & 0.000000 \\
\hline 4 & 6 & 0 & -0.648536 & -0.399437 & 0.000000 \\
\hline 5 & 1 & 0 & -1.568648 & 0.214185 & 0.000000 \\
\hline 6 & 8 & 0 & -0.648536 & -1.600954 & 0.000000 \\
\hline
\end{tabular}

FREQUENCIES AND ROTATIONAL CONSTANTS

\begin{tabular}{|c|c|c|c|c|c|}
\hline INDEX NO. & DOF TYPE & CM-1 (UNSCALED) & CM-1 (SCALED BY 1) & SYMMETRY & IR-INTENSITY \\
\hline 1 & vib & 132.06 & 132.06 & $\mathrm{AU}$ & 31.0029 \\
\hline 2 & vib & 337.03 & 337.03 & BU & 51.2026 \\
\hline 3 & vib & 552.42 & 552.42 & AG & 0.0000 \\
\hline 4 & vib & 816.15 & 816.15 & $\mathrm{AU}$ & 0.8809 \\
\hline 5 & vib & 1064.75 & 1064.75 & $\mathrm{AG}$ & 0.0000 \\
\hline 6 & vib & 1081.87 & 1081.87 & BG & 0.0000 \\
\hline 7 & vib & 1337.61 & 1337.61 & BU & 6.0694 \\
\hline 8 & vib & 1380.50 & 1380.50 & AG & 0.0000 \\
\hline 9 & vib & 1802.43 & 1802.43 & BU & 189.7208 \\
\hline 10 & vib & 1804.24 & 1804.24 & AG & 0.0000 \\
\hline 11 & vib & 2930.16 & 2930.16 & BU & 145.4509 \\
\hline 12 & vib & 2935.09 & 2935.09 & AG & 0.0000 \\
\hline 13 & rot & 1.8760599 & - & & \\
\hline 14 & rot & 0.1598839 & - & & \\
\hline 15 & rot & 0.1473281 & - & & \\
\hline
\end{tabular}

\section{HO2-freqs.log}

Charge $=0$ Multiplicity $=2$ Stoichiometry HO2(2)

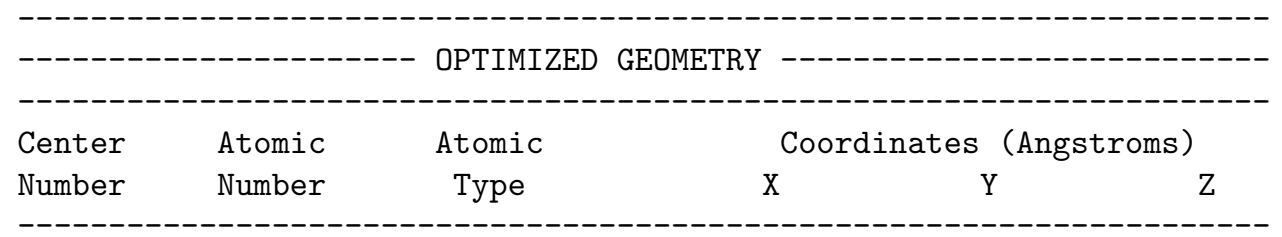




$\begin{array}{cccrrr}1 & 8 & 0 & 0.055373 & -0.610443 & 0.000000 \\ 2 & 1 & 0 & -0.885970 & -0.864927 & 0.000000 \\ 3 & 8 & 0 & 0.055373 & 0.718559 & 0.000000 \\ \text { Rotational constants (GHZ) : } & 619.9103554 & 33.6892980 & 31.9528087\end{array}$

FREQUENCIES AND ROTATIONAL CONSTANTS

INDEX NO. DOF TYPE CM-1(UNSCALED) CM-1(SCALED BY ) SYMMETRY IR-INTENSITY

$\begin{array}{llllll}2 & \text { vib } & 1432.88 & 0.00 & A^{\prime} & 41.4028\end{array}$

$\begin{array}{llllll}3 & \text { vib } & 3602.89 & 0.00 & A^{\prime} & 24.3131\end{array}$

$4 \quad$ rot $20.6779837 \quad-$

6 rot 1.0658310

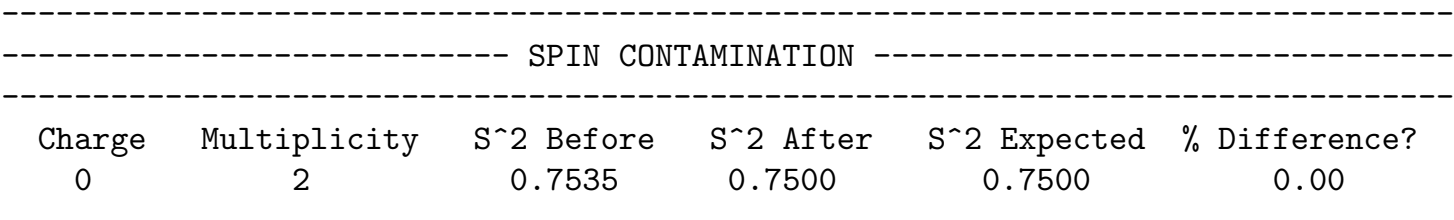

\section{Hydrazine.log}

Charge $=0$ Multiplicity $=1$ Stoichiometry H4N2

\begin{tabular}{|c|c|c|c|c|c|}
\hline \multirow{2}{*}{$\begin{array}{l}\text { Center } \\
\text { Number }\end{array}$} & \multirow{2}{*}{$\begin{array}{l}\text { Atomic } \\
\text { Number }\end{array}$} & \multirow{2}{*}{$\begin{array}{c}\text { Atomic } \\
\text { Type }\end{array}$} & \multicolumn{3}{|c|}{ Coordinates (Angstroms) } \\
\hline & & & $\mathrm{X}$ & $\mathrm{Y}$ & Z \\
\hline 1 & 7 & 0 & 0.000000 & 0.717787 & -0.075737 \\
\hline 2 & 7 & 0 & 0.000000 & -0.717787 & -0.075737 \\
\hline 3 & 1 & 0 & -0.230054 & 1.098142 & 0.836948 \\
\hline 4 & 1 & 0 & 0.230054 & -1.098142 & 0.836948 \\
\hline 5 & 1 & 0 & 0.936835 & 1.020404 & -0.306790 \\
\hline 6 & 1 & 0 & -0.936835 & -1.020404 & -0.306790 \\
\hline
\end{tabular}

FREQUENCIES AND ROTATIONAL CONSTANTS

$\begin{array}{ccccrr}\text { INDEX NO. DOF TYPE } & \text { CM-1 (UNSCALED) } & \text { CM-1(SCALED BY } & \text { 1) } & \text { SYMMETRY } & \text { IR-INTENSITY } \\ 1 & \text { vib } & 433.98 & 433.98 & \mathrm{~A} & 36.8945 \\ 2 & \text { vib } & 809.70 & 809.70 & \mathrm{~A} & 63.0610 \\ 3 & \text { vib } & 998.95 & 998.95 & \mathrm{~B} & 140.7688 \\ 4 & \text { vib } & 1112.39 & 1112.39 & \mathrm{~A} & 13.3289 \\ 5 & \text { vib } & 1300.35 & 1300.35 & \mathrm{~B} & 4.9458\end{array}$




\begin{tabular}{|c|c|c|c|c|c|}
\hline 6 & vib & 1331.87 & 1331.87 & $\mathrm{~A}$ & 3.9929 \\
\hline 7 & vib & 1675.16 & 1675.16 & B & 12.6698 \\
\hline 8 & vib & 1687.07 & 1687.07 & $\mathrm{~A}$ & 10.4305 \\
\hline 9 & vib & 3444.25 & 3444.25 & B & 15.2947 \\
\hline 10 & vib & 3454.90 & 3454.90 & $\mathrm{~A}$ & 3.5161 \\
\hline 11 & vib & 3550.83 & 3550.83 & $\mathrm{~A}$ & 0.0379 \\
\hline 12 & vib & 3557.64 & 3557.64 & B & 0.9762 \\
\hline 13 & rot & 4.8349794 & - & & \\
\hline 14 & rot & 0.8103356 & - & & \\
\hline 15 & rot & 0.8091229 & - & & \\
\hline
\end{tabular}

\section{Hydrogen-azide-HNNN.log}

Charge $=0$ Multiplicity $=1$ Stoichiometry HN3

\begin{tabular}{|c|c|c|c|c|c|}
\hline \multirow{2}{*}{$\begin{array}{l}\text { Center } \\
\text { Number }\end{array}$} & \multirow{2}{*}{$\begin{array}{l}\text { Atomic } \\
\text { Number }\end{array}$} & \multirow{2}{*}{$\begin{array}{c}\text { Atomic } \\
\text { Type }\end{array}$} & \multicolumn{3}{|c|}{ Coordinates (Angstroms) } \\
\hline & & & $\mathrm{X}$ & $\mathrm{Y}$ & $\mathrm{Z}$ \\
\hline 1 & 7 & 0 & 0.076802 & -1.121924 & 0.000000 \\
\hline 2 & 7 & 0 & 0.000000 & 0.110596 & 0.000000 \\
\hline 3 & 7 & 0 & -0.226907 & 1.213788 & 0.000000 \\
\hline 4 & 1 & 0 & 1.050737 & -1.417220 & 0.000000 \\
\hline
\end{tabular}

FREQUENCIES AND ROTATIONAL CONSTANTS

INDEX NO. DOF TYPE CM-1(UNSCALED) CM-1(SCALED BY) SYMMETRY IR-INTENSITY

$\begin{array}{rrrrrr}1 & \text { vib } & 550.32 & 0.00 & A^{\prime} & 14.9605 \\ 2 & \text { vib } & 620.26 & 0.00 & A^{\prime \prime} & 0.0177 \\ 3 & \text { vib } & 1186.52 & 0.00 & A^{\prime} & 217.9847 \\ 4 & \text { vib } & 1303.43 & 0.00 & A^{\prime} & 2.8238 \\ 5 & \text { vib } & 2277.76 & 0.00 & A^{\prime} & 364.0478 \\ 6 & \text { vib } & 3488.08 & 0.00 & A^{\prime} & 42.6760 \\ 7 & \text { rot } & 20.7101531 & - & & \\ 8 & \text { rot } & 0.4063764 & - & & \\ 9 & \text { rot } & 0.3985559 & - & & \end{array}$

\section{Hydrogen-isocyanide.log}

Charge $=0$ Multiplicity $=1$ Stoichiometry CHN 


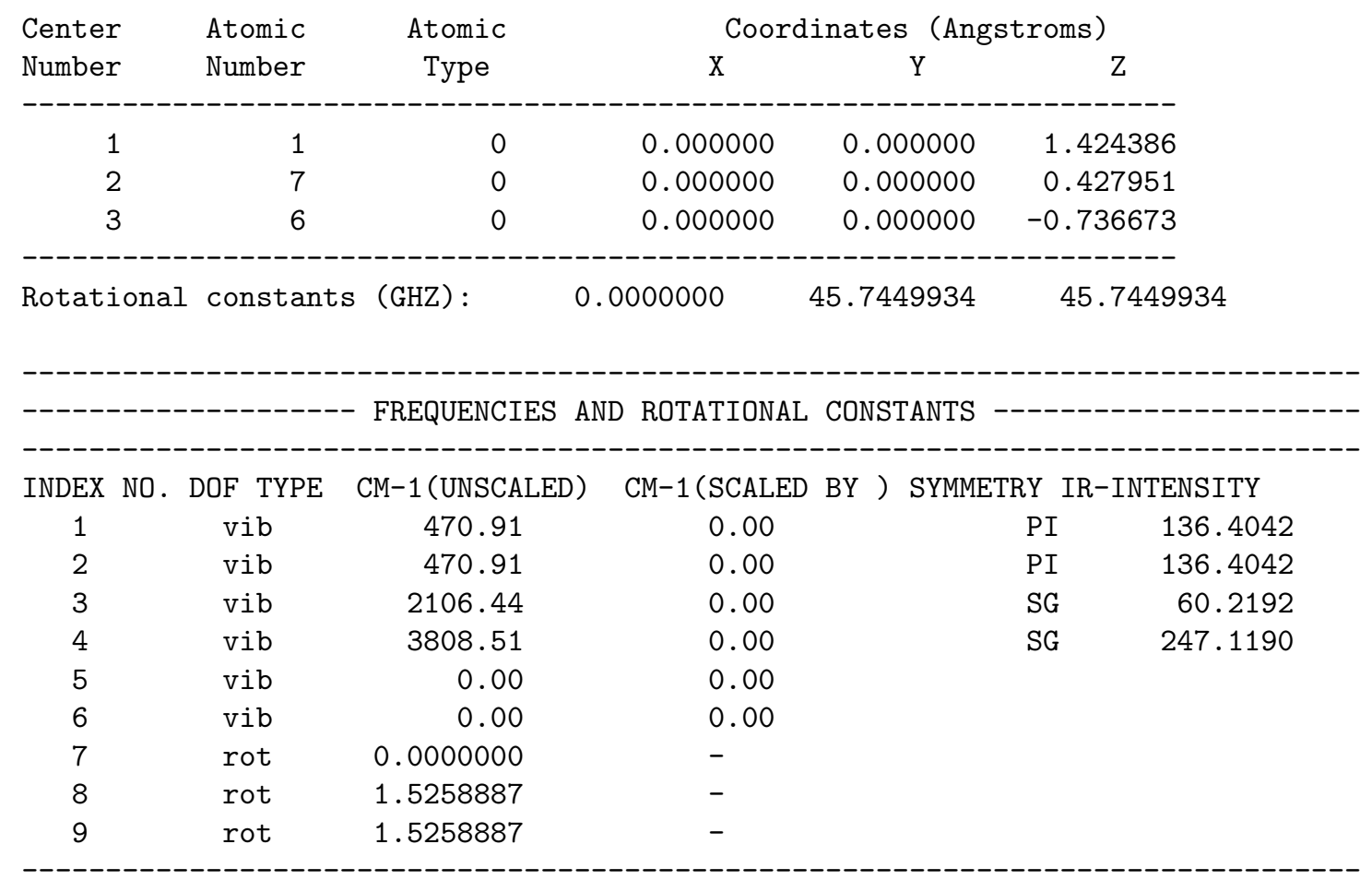

\section{Hydroxyamidogen-cis.log}

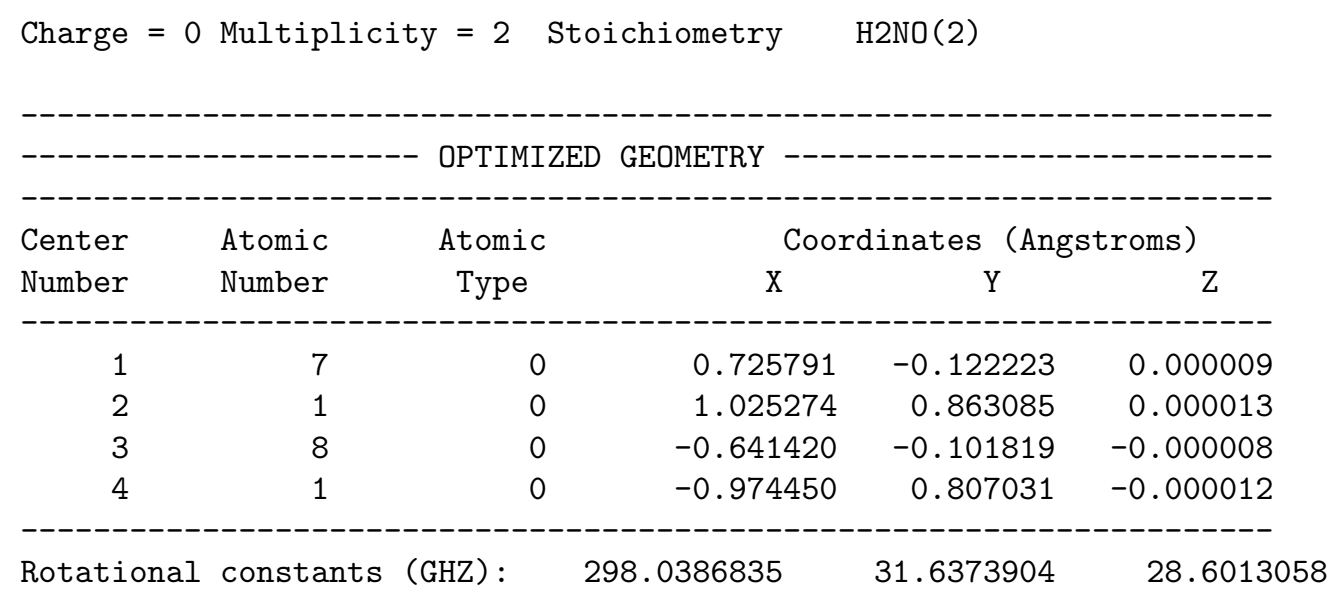

\section{FREQUENCIES AND ROTATIONAL CONSTANTS}

INDEX NO. DOF TYPE CM-1(UNSCALED) CM-1(SCALED BY) SYMMETRY IR-INTENSITY

\begin{tabular}{|c|c|c|c|c|c|}
\hline 1 & vib & 562.94 & 0.00 & $\mathrm{~A}$ & 5.3258 \\
\hline 2 & vib & 1092.19 & 0.00 & $\mathrm{~A}$ & 53.3182 \\
\hline 3 & vib & 1324.05 & 0.00 & $\mathrm{~A}$ & 3.8537 \\
\hline 4 & vib & 1514.05 & 0.00 & $\mathrm{~A}$ & 49.5750 \\
\hline 5 & vib & 3300.39 & 0.00 & $\mathrm{~A}$ & 24.2945 \\
\hline 6 & vib & 3707.47 & 0.00 & A & 19.5419 \\
\hline 7 & rot & 9.9415004 & - & & \\
\hline 8 & rot & 1.0553098 & - & & \\
\hline
\end{tabular}




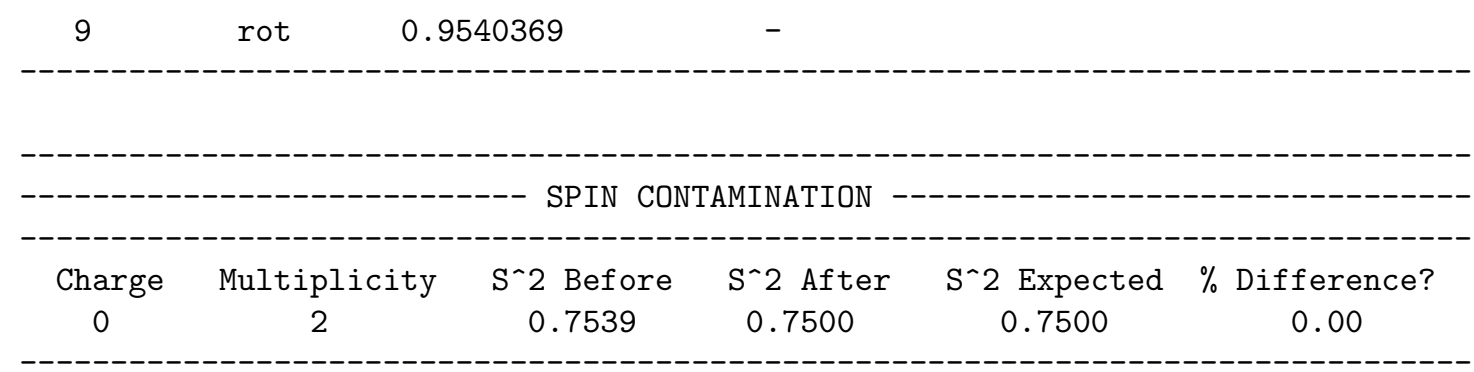

\section{Hydroxyamidogen-trans.log}

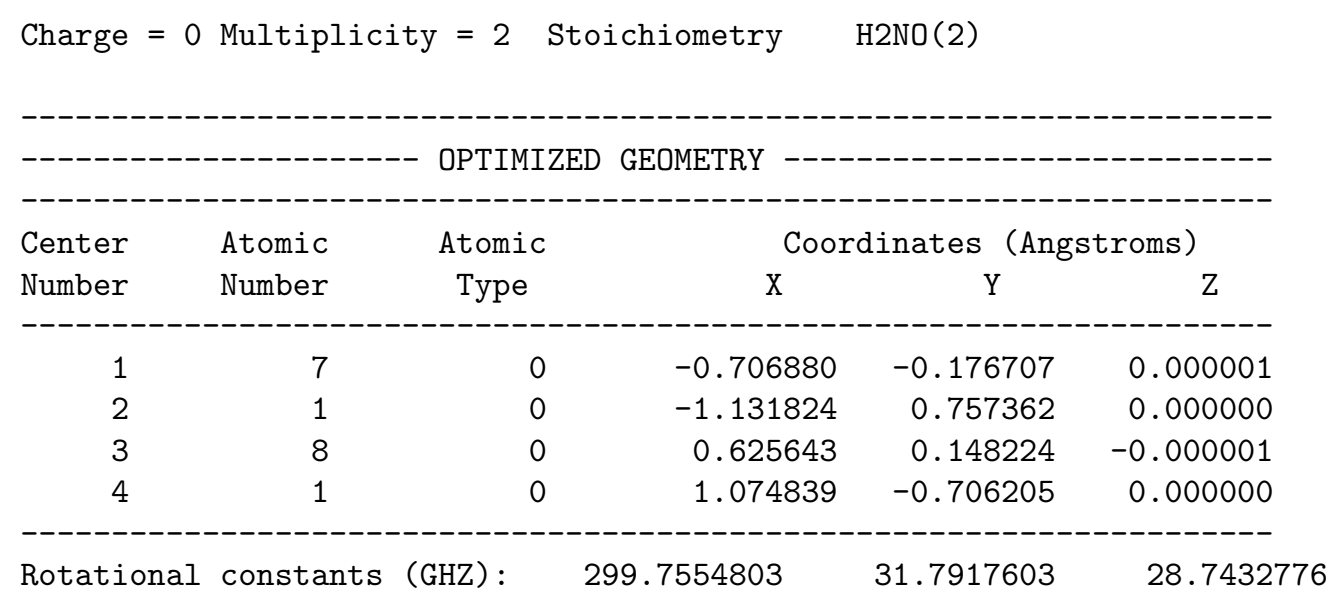

FREQUENCIES AND ROTATIONAL CONSTANTS

$\begin{array}{cccccr}\text { INDEX NO. DOF TYPE } & \text { CM-1(UNSCALED) } & \text { CM-1 (SCALED BY ) } & \text { SYMMETRY IR-INTENSITY } \\ 1 & \text { vib } & 742.94 & 0.00 & \mathrm{~A} & 188.4601 \\ 2 & \text { vib } & 1104.06 & 0.00 & \mathrm{~A} & 59.0808 \\ 3 & \text { vib } & 1269.14 & 0.00 & \mathrm{~A} & 106.8232 \\ 4 & \text { vib } & 1576.45 & 0.00 & \mathrm{~A} & 1.7267 \\ 5 & \text { vib } & 3367.00 & 0.00 & \mathrm{~A} & 6.3001 \\ 6 & \text { vib } & 3768.08 & 0.00 & \mathrm{~A} & 64.1726 \\ 7 & \text { rot } & 9.9987666 & - & & \\ 8 & \text { rot } & 1.0604590 & - & & \\ 9 & \text { rot } & 0.9587725 & - & \end{array}$

\begin{tabular}{|c|c|c|c|c|c|}
\hline Charge & Multiplicity & $\mathrm{S}^{\wedge} 2$ Before & $\mathrm{S}^{\wedge} 2$ After & $\mathrm{S}^{\wedge} 2$ Expected & $\%$ Difference? \\
\hline 0 & 2 & 0.7538 & 0.7500 & 0.7500 & 0.00 \\
\hline
\end{tabular}




\section{Hydroxyformyl-cis.log}

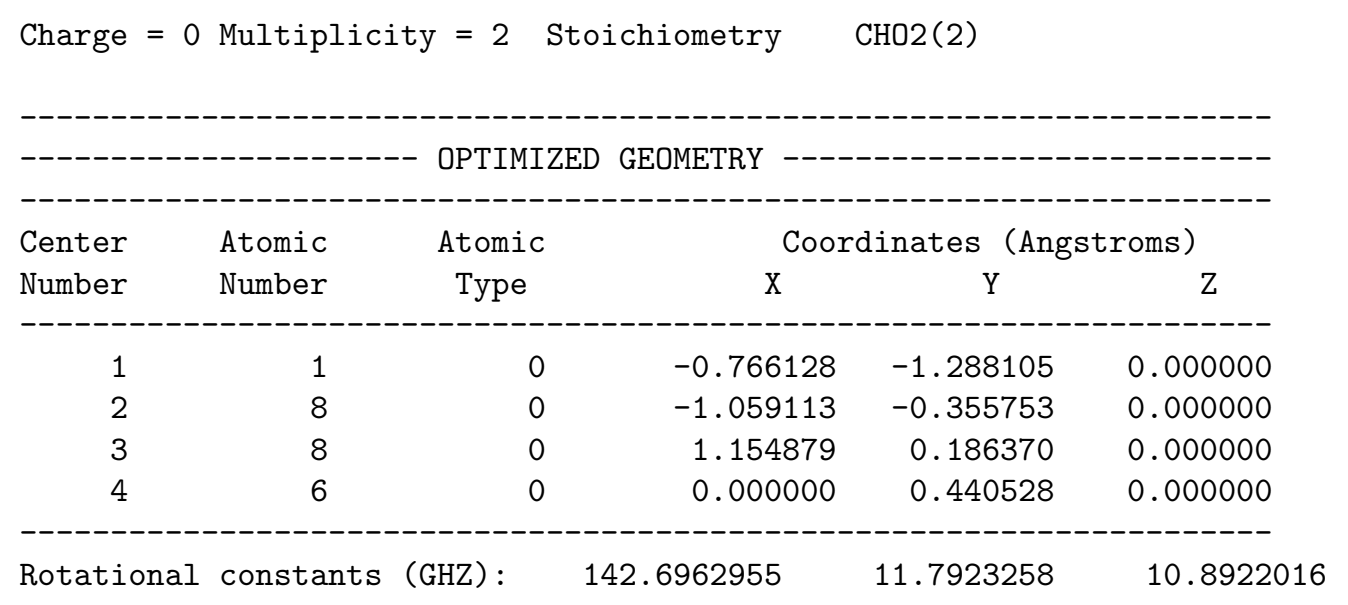

FREQUENCIES AND ROTATIONAL CONSTANTS

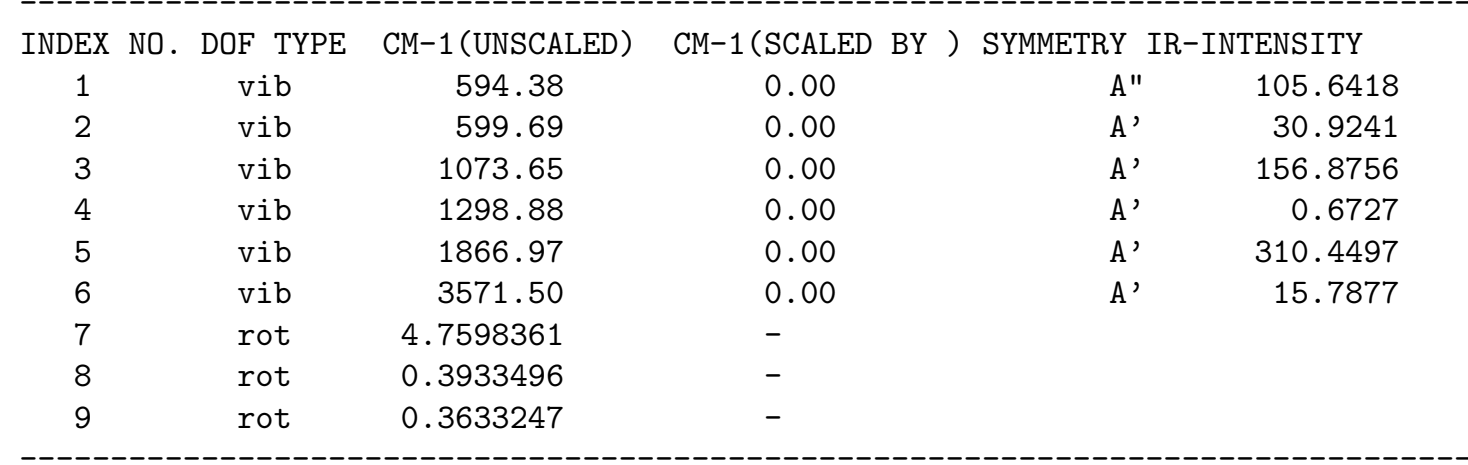

\begin{tabular}{|c|c|c|c|c|c|}
\hline Charge & Multiplicity & $\mathrm{S}^{\wedge} 2$ Before & $S^{\wedge} 2$ After & $\mathrm{S}^{\wedge} 2$ Expected & $\%$ Difference? \\
\hline 0 & 2 & 0.7531 & 0.7500 & 0.7500 & 0.00 \\
\hline
\end{tabular}

\section{Hydroxyformyl-trans.log}

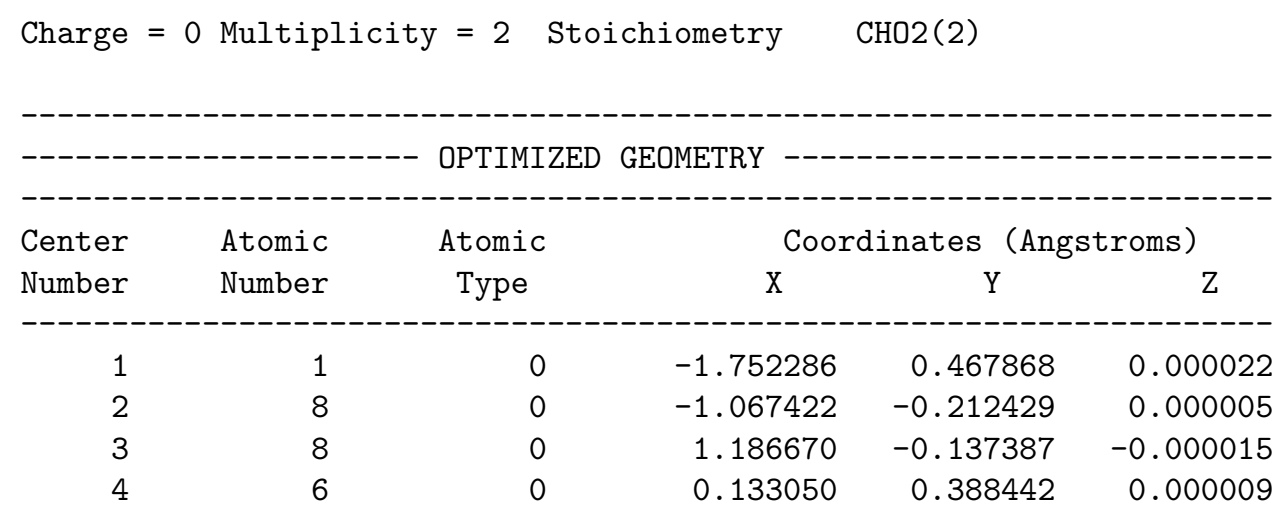


$\begin{array}{llll}\text { Rotational constants (GHZ): } & 166.6079742 & 11.4845954 & 10.7439922\end{array}$

FREQUENCIES AND ROTATIONAL CONSTANTS

$\begin{array}{cccccr}\text { INDEX NO. DOF TYPE } & \text { CM-1(UNSCALED) } & \text { CM-1 (SCALED BY ) } & \text { SYMMETRY } & \text { IR-INTENSITY } \\ 1 & \text { vib } & 545.27 & 0.00 & \text { A } & 86.3819 \\ 2 & \text { vib } & 622.11 & 0.00 & \text { A } & 4.2341 \\ 3 & \text { vib } & 1084.35 & 0.00 & \text { A } & 71.9037 \\ 4 & \text { vib } & 1242.65 & 0.00 & \text { A } & 242.6044 \\ 5 & \text { vib } & 1902.60 & 0.00 & \text { A } & 217.1277 \\ 6 & \text { vib } & 3793.66 & 0.00 & & 118.3432 \\ 7 & \text { rot } & 5.5574438 & - & & \\ 8 & \text { rot } & 0.3830849 & - & & \\ 9 & \text { rot } & 0.3583810 & - & & \end{array}$

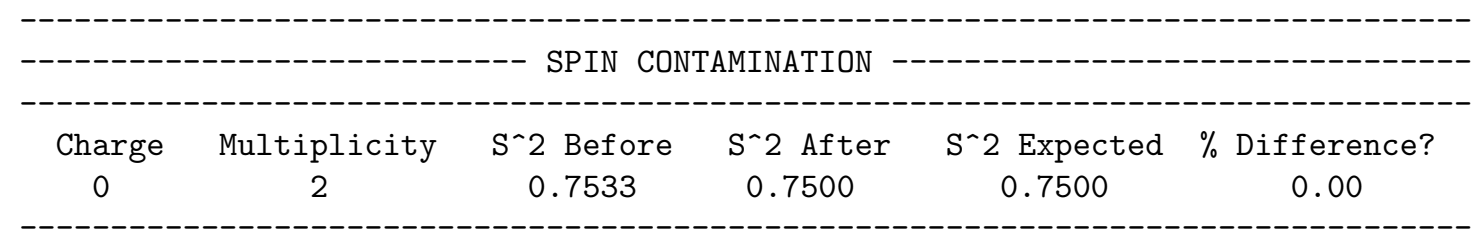

\section{Hydroxylamine-cis.log}

$$
\text { Charge }=0 \text { Multiplicity }=1 \text { Stoichiometry H3NO }
$$

\begin{tabular}{|c|c|c|c|c|c|}
\hline \multirow{2}{*}{$\begin{array}{l}\text { Center } \\
\text { Number }\end{array}$} & \multirow{2}{*}{$\begin{array}{l}\text { Atomic } \\
\text { Number }\end{array}$} & \multirow{2}{*}{$\begin{array}{l}\text { Atomic } \\
\text { Type }\end{array}$} & \multicolumn{3}{|c|}{ Coordinates (Angstroms) } \\
\hline & & & $\mathrm{X}$ & $\mathrm{Y}$ & $\mathrm{Z}$ \\
\hline 1 & 7 & 0 & -0.108795 & 0.699659 & 0.000000 \\
\hline 2 & 1 & 0 & 0.410766 & 1.002373 & 0.820045 \\
\hline 3 & 1 & 0 & 0.410766 & 1.002373 & -0.820045 \\
\hline 4 & 8 & 0 & -0.108795 & -0.733854 & 0.000000 \\
\hline 5 & 1 & 0 & 0.810386 & -1.031526 & 0.000000 \\
\hline
\end{tabular}

Rotational constants (GHZ): $192.0280806 \quad 25.5034462 \quad 25.4019882$

FREQUENCIES AND ROTATIONAL CONSTANTS

INDEX NO. DOF TYPE CM-1(UNSCALED) CM-1(SCALED BY ) SYMMETRY IR-INTENSITY

\begin{tabular}{|c|c|c|c|c|c|}
\hline 1 & vib & 463.09 & 0.00 & $\mathrm{~A}^{\prime \prime}$ & 27.3645 \\
\hline 2 & vib & 910.41 & 0.00 & $A^{\prime}$ & 20.8947 \\
\hline 3 & vib & 1146.78 & 0.00 & $A^{\prime}$ & 56.8316 \\
\hline 4 & vib & 1320.74 & 0.00 & $A^{\prime \prime}$ & 0.1703 \\
\hline 5 & vib & 1372.50 & 0.00 & $A^{\prime}$ & 27.1739 \\
\hline 6 & vib & 1646.72 & 0.00 & $A^{\prime}$ & 19.1395 \\
\hline 7 & vib & 3413.02 & 0.00 & $A^{\prime}$ & 6.2436 \\
\hline
\end{tabular}




$\begin{array}{rrrlll}8 & \text { vib } & 3512.86 & 0.00 & \text { A" } & 0.1186 \\ 9 & \text { vib } & 3718.55 & 0.00 & \text { A } & 4.8819 \\ 10 & \text { rot } & 6.4053673 & - & \\ 11 & \text { rot } & 0.8507034 & - & \\ 12 & \text { rot } & 0.8473191 & - & \end{array}$

\section{Hydroxylamine-trans.log}

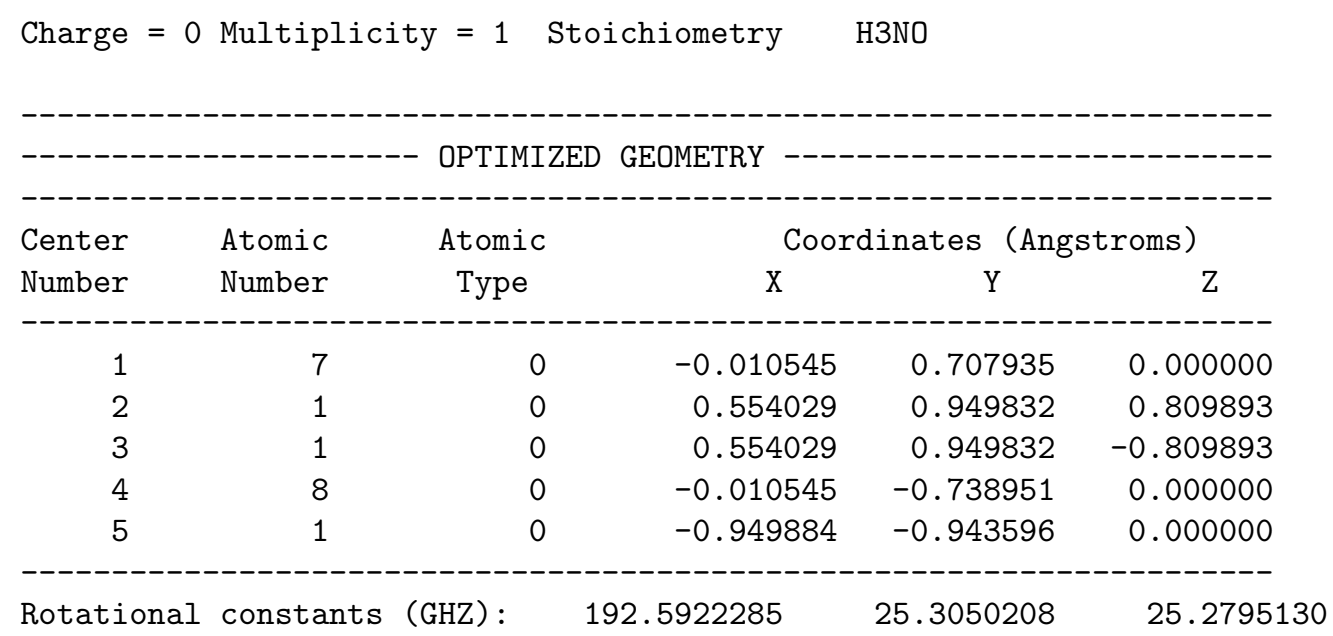

\section{FREQUENCIES AND ROTATIONAL CONSTANTS}

INDEX NO. DOF TYPE CM-1(UNSCALED) CM-1(SCALED BY) SYMMETRY IR-INTENSITY

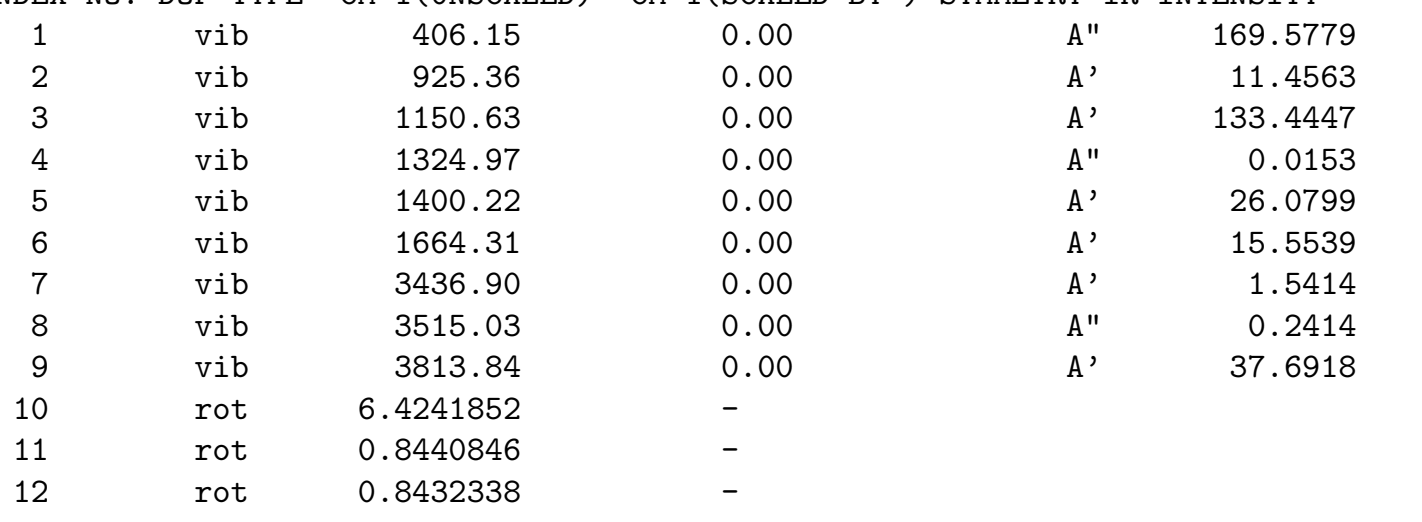

\section{Hydroxymethylene-cis.log}

Charge $=0$ Multiplicity $=1$ Stoichiometry $\quad$ CH2O

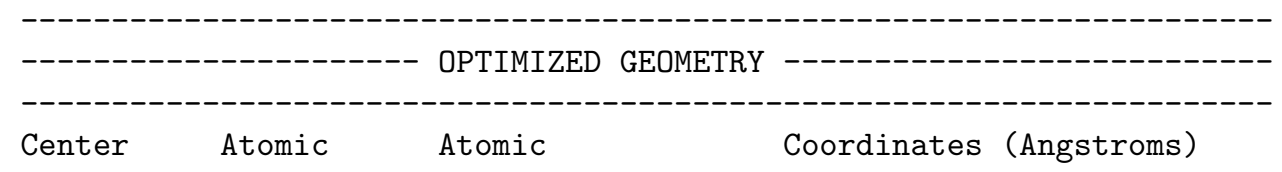




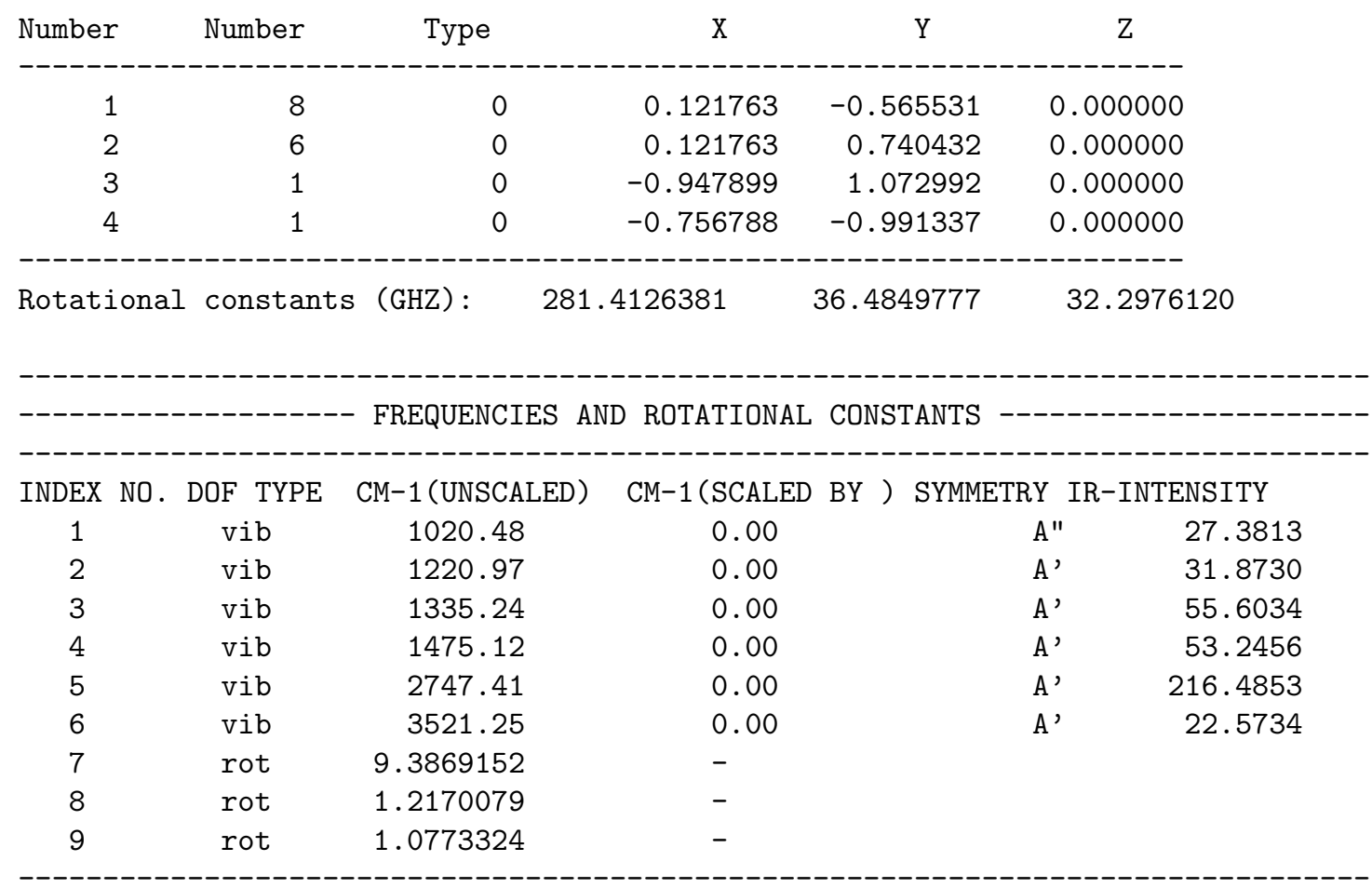

\section{Hydroxymethylene-g-triplet.log}

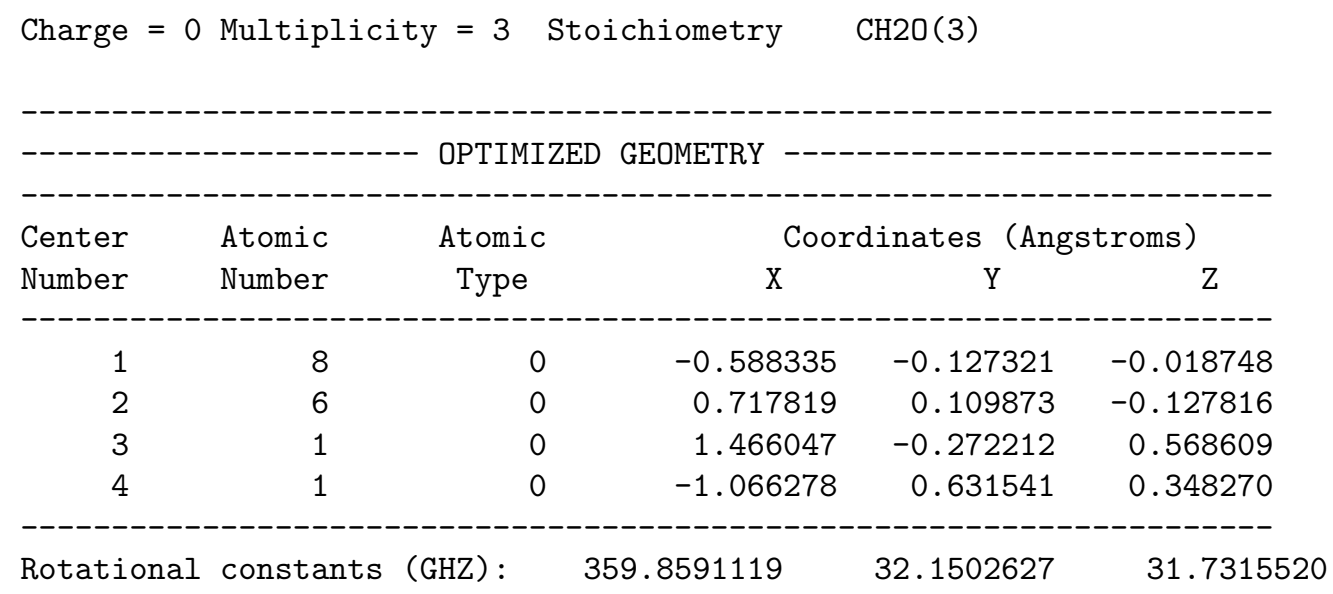

FREQUENCIES AND ROTATIONAL CONSTANTS

INDEX NO. DOF TYPE CM-1(UNSCALED) CM-1(SCALED BY) SYMMETRY IR-INTENSITY

$\begin{array}{rrrrrr}1 & \text { vib } & 457.30 & 0.00 & \mathrm{~A} & 78.6570 \\ 2 & \text { vib } & 1077.82 & 0.00 & \mathrm{~A} & 86.3160 \\ 3 & \text { vib } & 1155.59 & 0.00 & \mathrm{~A} & 70.9852 \\ 4 & \text { vib } & 1301.89 & 0.00 & \mathrm{~A} & 23.6065 \\ 5 & \text { vib } & 3050.85 & 0.00 & \mathrm{~A} & 19.9513 \\ 6 & \text { vib } & 3657.29 & 0.00 & \mathrm{~A} & 37.1778 \\ 7 & \text { rot } & 12.0036079 & - & & \\ 8 & \text { rot } & 1.0724173 & - & & \end{array}$




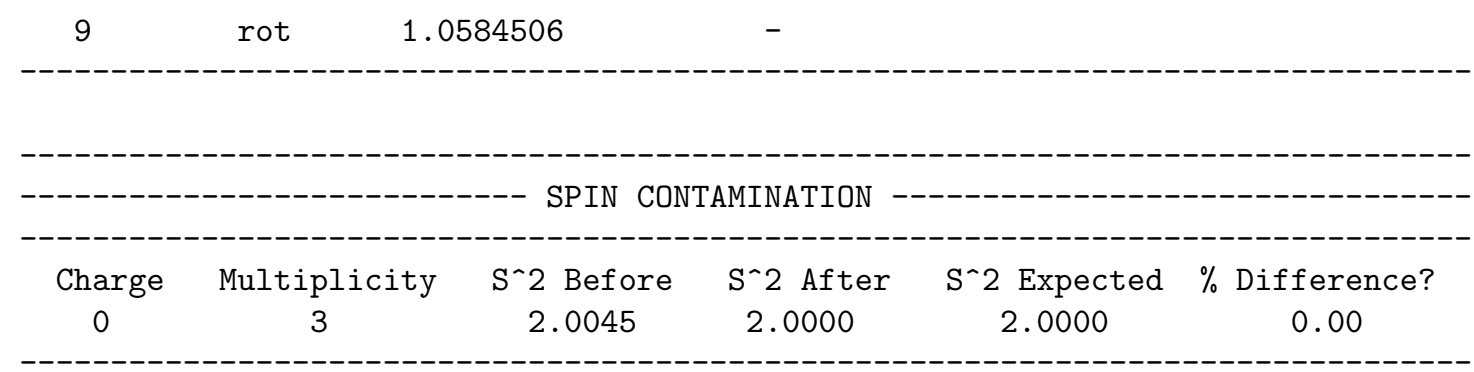

\section{$76 \quad$ Hydroxymethylene-trans.log}

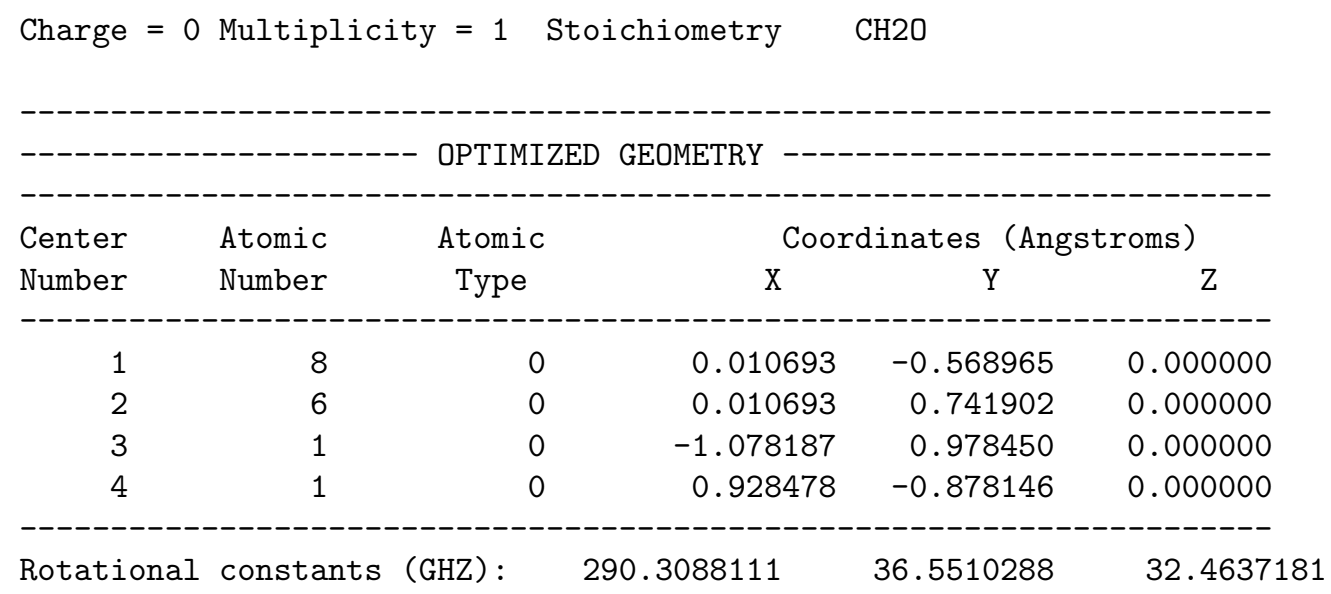

\section{FREQUENCIES AND ROTATIONAL CONSTANTS}

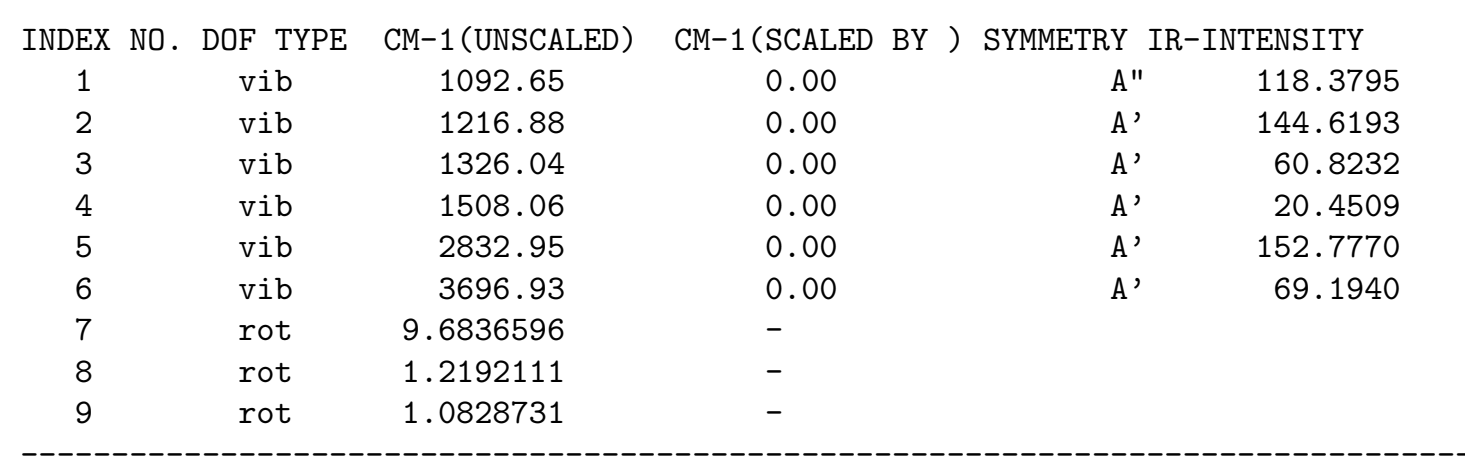

\section{$77 \quad$ Iminomethyl-cis.log}

Charge $=0$ Multiplicity $=2$ Stoichiometry CH2N(2)

$\begin{array}{lccc}\text { Center } & \text { Atomic } & \text { Atomic } & \text { OPTIMIZED GEOMETRY } \\ \text { Number } & \text { Number } & \text { Type } & \text { X Coordinates (Angstroms) }\end{array}$




$\begin{array}{cccrrr}1 & 6 & 0 & -0.633024 & -0.121687 & 0.000006 \\ 2 & 7 & 0 & 0.584339 & -0.099907 & -0.000006 \\ 3 & 1 & 0 & -1.410120 & 0.653290 & 0.000014 \\ 4 & 1 & 0 & 1.117896 & 0.776182 & -0.000012 \\ \text { Rotational constants (GHZ) : } & 394.0438903 & 39.3265468 & 35.7578279\end{array}$

FREQUENCIES AND ROTATIONAL CONSTANTS

$\begin{array}{cccccr}\text { INDEX NO. DOF TYPE } & \text { CM-1(UNSCALED) } & \text { CM-1(SCALED BY ) } & \text { SYMMETRY } & \text { IR-INTENSITY } \\ 1 & \text { vib } & 872.72 & 0.00 & \mathrm{~A} & 106.2974 \\ 2 & \text { vib } & 905.11 & 0.00 & \mathrm{~A} & 4.6483 \\ 3 & \text { vib } & 1003.67 & 0.00 & \mathrm{~A} & 154.8062 \\ 4 & \text { vib } & 1861.92 & 0.00 & \mathrm{~A} & 26.1861 \\ 5 & \text { vib } & 2957.22 & 0.00 & \mathrm{~A} & 51.5186 \\ 6 & \text { vib } & 3293.93 & 0.00 & \mathrm{~A} & 3.5858 \\ 7 & \text { rot } & 13.1438894 & - & & \\ 8 & \text { rot } & 1.3117924 & - & & \\ 9 & \text { rot } & 1.1927528 & - & & \end{array}$

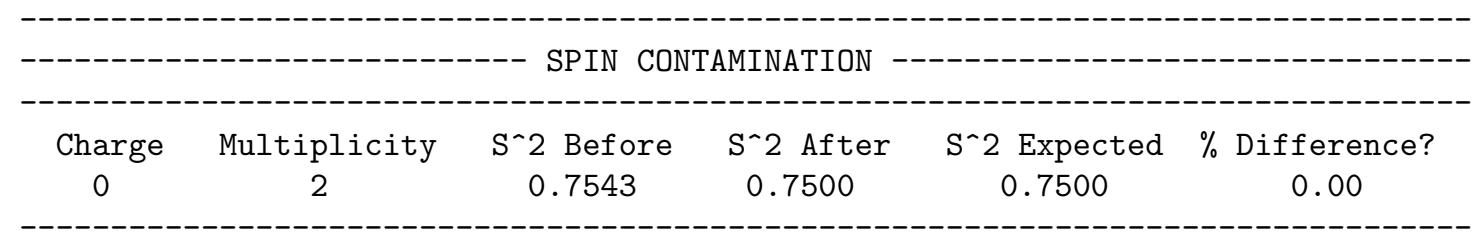

\section{Iminomethyl-trans.log}

Charge $=0$ Multiplicity $=2$ Stoichiometry $\quad$ CH2N(2)

\begin{tabular}{|c|c|c|c|c|c|}
\hline \multirow{2}{*}{$\begin{array}{l}\text { Center } \\
\text { Number }\end{array}$} & \multirow{2}{*}{$\begin{array}{l}\text { Atomic } \\
\text { Number }\end{array}$} & \multirow{2}{*}{$\begin{array}{l}\text { Atomic } \\
\text { Type }\end{array}$} & \multicolumn{3}{|c|}{ Coordinates (Angstroms) } \\
\hline & & & $\mathrm{X}$ & $\mathrm{Y}$ & $\mathrm{Z}$ \\
\hline 1 & 6 & 0 & 0.616522 & 0.193618 & 0.000000 \\
\hline 2 & 7 & 0 & -0.555550 & -0.173647 & 0.000000 \\
\hline 3 & 1 & 0 & 1.484158 & -0.475720 & 0.000001 \\
\hline 4 & 1 & 0 & -1.294441 & 0.529542 & 0.000001 \\
\hline
\end{tabular}

FREQUENCIES AND ROTATIONAL CONSTANTS

INDEX NO. DOF TYPE CM-1(UNSCALED) CM-1(SCALED BY) SYMMETRY IR-INTENSITY

$\begin{array}{llllll}1 & \text { vib } & 912.43 & 0.00 & A & 221.6583 \\ 2 & \text { vib } & 970.85 & 0.00 & A & 110.3207\end{array}$




$\begin{array}{lrrllr}3 & \text { vib } & 1192.12 & 0.00 & \text { A } & 11.9025 \\ 4 & \text { vib } & 1803.18 & 0.00 & \text { A } & 26.9867 \\ 5 & \text { vib } & 3001.29 & 0.00 & \text { A } & 28.5279 \\ 6 & \text { vib } & 3419.54 & 0.00 & \text { A } & 5.5739 \\ 7 & \text { rot } & 13.8406083 & - & & \\ 8 & \text { rot } & 1.3012353 & - & & \\ 9 & \text { rot } & 1.1894119 & - & \\ -\end{array}$

SPIN CONTAMINATION

$\begin{array}{cccccc}\text { Charge } & \text { Multiplicity } & S^{\wedge} 2 \text { Before } & S^{\wedge} 2 \text { After } & S^{\wedge} 2 \text { Expected } & \% \text { Difference? } \\ 0 & 2 & 0.7534 & 0.7500 & 0.7500 & 0.00\end{array}$

\section{Isobutane-freqs.log}

\begin{tabular}{|c|c|c|c|c|c|}
\hline \multicolumn{6}{|c|}{ Multipl } \\
\hline \multicolumn{6}{|c|}{ OPTIMIZED GEOMETRY } \\
\hline \multirow{2}{*}{$\begin{array}{l}\text { Center } \\
\text { Number }\end{array}$} & \multirow{2}{*}{$\begin{array}{l}\text { Atomic } \\
\text { Number }\end{array}$} & \multirow{2}{*}{$\begin{array}{c}\text { Atomic } \\
\text { Type }\end{array}$} & \multicolumn{2}{|c|}{ Coordinates (Angstroms) } & troms) \\
\hline & & & $\mathrm{X}$ & $\mathrm{Y}$ & $\mathrm{Z}$ \\
\hline 1 & 6 & 0 & -0.371337 & 0.000010 & 0.000000 \\
\hline 2 & 1 & 0 & -1.466625 & 0.000022 & 0.000000 \\
\hline 3 & 6 & 0 & 0.095461 & 1.457997 & 0.000000 \\
\hline 4 & 6 & 0 & 0.095461 & -0.728997 & 1.262642 \\
\hline 5 & 6 & 0 & 0.095461 & -0.728997 & -1.262642 \\
\hline 6 & 1 & 0 & 1.187128 & 1.515779 & 0.000000 \\
\hline 7 & 1 & 0 & 1.187129 & -0.757839 & 1.312706 \\
\hline 8 & 1 & 0 & 1.187129 & -0.757839 & -1.312706 \\
\hline 9 & 1 & 0 & -0.264169 & 1.990796 & 0.882178 \\
\hline 10 & 1 & 0 & -0.264169 & 1.990796 & -0.882178 \\
\hline 11 & 1 & 0 & -0.264124 & -1.759406 & 1.282929 \\
\hline 12 & 1 & 0 & -0.264228 & -0.231487 & 2.165172 \\
\hline 13 & 1 & 0 & -0.264228 & -0.231487 & -2.165172 \\
\hline 14 & 1 & 0 & -0.264124 & -1.759406 & -1.282929 \\
\hline
\end{tabular}

FREQUENCIES AND ROTATIONAL CONSTANTS

\begin{tabular}{|c|c|c|c|c|c|}
\hline INDEX NO. & DOF TYPE & CM-1 (UNSCALED) & $\mathrm{CM}-1$ (SCALED BY 1$)$ & SYMMETRY & IR-INTENSITY \\
\hline 1 & vib & 214.10 & 214.10 & A" & 0.0000 \\
\hline 2 & vib & 254.90 & 254.90 & A" & 0.0120 \\
\hline 3 & vib & 255.53 & 255.53 & $A^{\prime}$ & 0.0121 \\
\hline 4 & vib & 364.24 & 364.24 & $A^{\prime}$ & 0.0118 \\
\hline 5 & vib & 364.46 & 364.46 & A" & 0.0118 \\
\hline 6 & vib & 431.21 & 431.21 & $A^{\prime}$ & 0.2020 \\
\hline
\end{tabular}




\begin{tabular}{|c|c|c|c|c|c|}
\hline 7 & vib & 795.04 & 795.04 & $A^{\prime}$ & 0.5561 \\
\hline 8 & vib & 926.05 & 926.05 & A" & 0.8766 \\
\hline 9 & vib & 926.06 & 926.06 & $A^{\prime}$ & 0.8765 \\
\hline 10 & vib & 959.41 & 959.41 & A" & 0.0000 \\
\hline 11 & vib & 971.88 & 971.88 & $\mathrm{~A}^{\prime \prime}$ & 0.0007 \\
\hline 12 & vib & 971.93 & 971.93 & $A^{\prime}$ & 0.0007 \\
\hline 13 & vib & 1192.83 & 1192.83 & $A^{\prime}$ & 2.7585 \\
\hline 14 & vib & 1192.86 & 1192.86 & $\mathrm{~A}^{\prime \prime}$ & 2.7546 \\
\hline 15 & vib & 1212.36 & 1212.36 & $A^{\prime}$ & 0.1272 \\
\hline 16 & vib & 1362.50 & 1362.50 & $A^{\prime}$ & 3.4603 \\
\hline 17 & vib & 1362.53 & 1362.53 & A" & 3.4557 \\
\hline 18 & vib & 1402.93 & 1402.93 & $A^{\prime}$ & 6.2155 \\
\hline 19 & vib & 1402.99 & 1402.99 & $\mathrm{~A}^{\prime \prime}$ & 6.2286 \\
\hline 20 & vib & 1431.19 & 1431.19 & $A^{\prime}$ & 3.4135 \\
\hline 21 & vib & 1484.19 & 1484.19 & $\mathrm{~A}^{\prime \prime}$ & 0.0000 \\
\hline 22 & vib & 1490.75 & 1490.75 & $A^{\prime}$ & 0.3961 \\
\hline 23 & vib & 1490.84 & 1490.84 & A" & 0.3909 \\
\hline 24 & vib & 1508.14 & 1508.14 & A" & 3.8961 \\
\hline 25 & vib & 1508.19 & 1508.19 & $A^{\prime}$ & 3.8930 \\
\hline 26 & vib & 1515.47 & 1515.47 & $A^{\prime}$ & 16.8796 \\
\hline 27 & vib & 2993.59 & 2993.59 & $A^{\prime}$ & 11.6902 \\
\hline 28 & vib & 3012.47 & 3012.47 & A" & 34.4854 \\
\hline 29 & vib & 3012.49 & 3012.49 & $A^{\prime}$ & 34.4787 \\
\hline 30 & vib & 3019.94 & 3019.94 & $A^{\prime}$ & 16.5737 \\
\hline 31 & vib & 3069.02 & 3069.02 & $\mathrm{~A}^{\prime \prime}$ & 7.3918 \\
\hline 32 & vib & 3069.04 & 3069.04 & $A^{\prime}$ & 7.4298 \\
\hline 33 & vib & 3078.54 & 3078.54 & A" & 0.0083 \\
\hline 34 & vib & 3079.04 & 3079.04 & $A^{\prime}$ & 102.3503 \\
\hline 35 & vib & 3082.46 & 3082.46 & $A^{\prime}$ & 58.9514 \\
\hline 36 & vib & 3082.51 & 3082.51 & A" & 58.9745 \\
\hline 37 & rot & 0.2597463 & - & & \\
\hline 38 & rot & 0.2597403 & - & & \\
\hline 39 & rot & 0.1503212 & - & & \\
\hline
\end{tabular}

\section{IsocyanicAcid-HNCO.log}

Charge $=0$ Multiplicity $=1$ Stoichiometry CHNO

\begin{tabular}{|c|c|c|c|c|c|}
\hline \multirow{2}{*}{$\begin{array}{l}\text { Center } \\
\text { Number }\end{array}$} & \multirow{2}{*}{$\begin{array}{l}\text { Atomic } \\
\text { Number }\end{array}$} & \multirow{2}{*}{$\begin{array}{c}\text { Atomic } \\
\text { Type }\end{array}$} & \multicolumn{3}{|c|}{ Coordinates (Angstroms) } \\
\hline & & & $\mathrm{X}$ & $\mathrm{Y}$ & $\mathrm{Z}$ \\
\hline 1 & 1 & 0 & 1.340371 & -1.393609 & 0.000000 \\
\hline 2 & 7 & 0 & 0.379494 & -1.100503 & 0.000000 \\
\hline 3 & 6 & 0 & 0.000000 & 0.048759 & 0.000000 \\
\hline 4 & 8 & 0 & -0.499603 & 1.100572 & 0.000000 \\
\hline
\end{tabular}




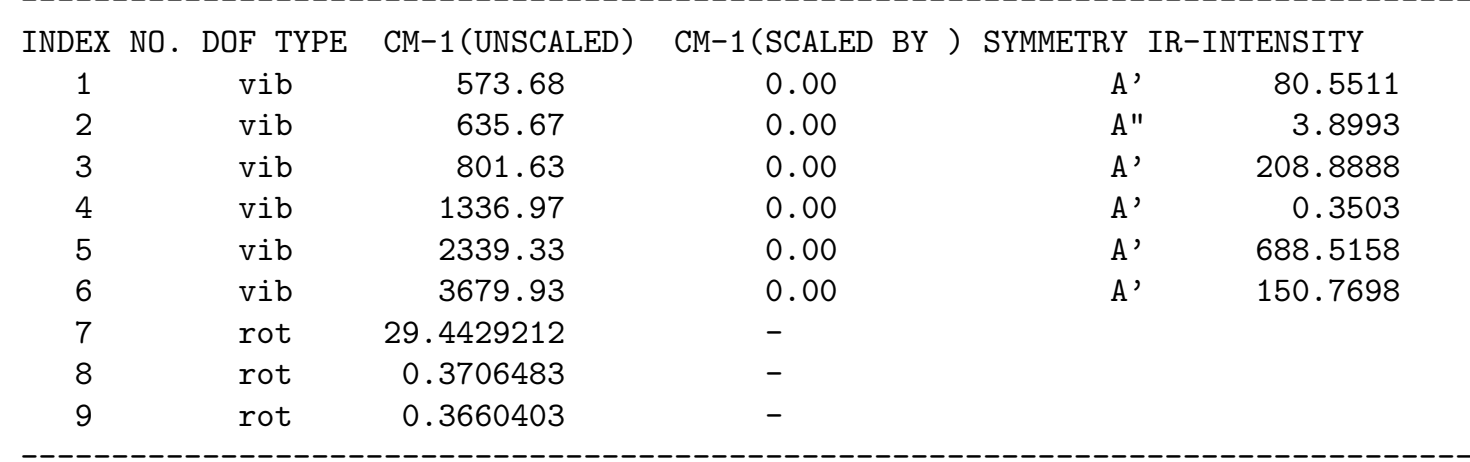

\section{Isocyanogen.log}

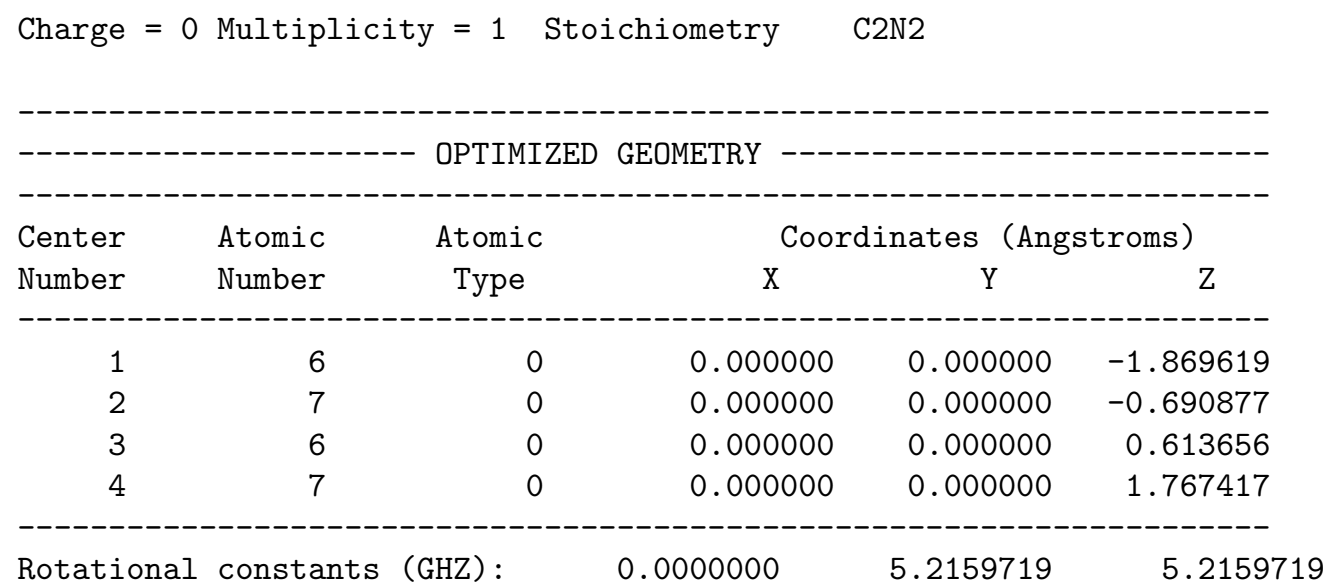

FREQUENCIES AND ROTATIONAL CONSTANTS

INDEX NO. DOF TYPE CM-1(UNSCALED) CM-1(SCALED BY) SYMMETRY IR-INTENSITY

$\begin{array}{rrrrrr}1 & \text { vib } & 214.49 & 0.00 & \mathrm{PI} & 9.3307 \\ 2 & \text { vib } & 214.49 & 0.00 & \mathrm{PI} & 9.3307 \\ 3 & \text { vib } & 508.64 & 0.00 & \mathrm{PI} & 7.3582 \\ 4 & \text { vib } & 508.64 & 0.00 & \mathrm{PI} & 7.3582 \\ 5 & \text { vib } & 960.44 & 0.00 & \mathrm{SG} & 0.6358 \\ 6 & \text { vib } & 2134.03 & 0.00 & \mathrm{SG} & 156.5266 \\ 7 & \text { vib } & 2393.80 & 0.00 & \mathrm{SG} & 107.5098 \\ 8 & \text { vib } & 0.00 & 0.00 & & \\ 9 & \text { vib } & 0.00 & 0.00 & & \\ 10 & \text { rot } & 0.0000000 & - & & \\ 11 & \text { rot } & 0.1739861 & - & & \\ 12 & \text { rot } & 0.1739861 & - & \end{array}$




\section{Isodiazine.log}

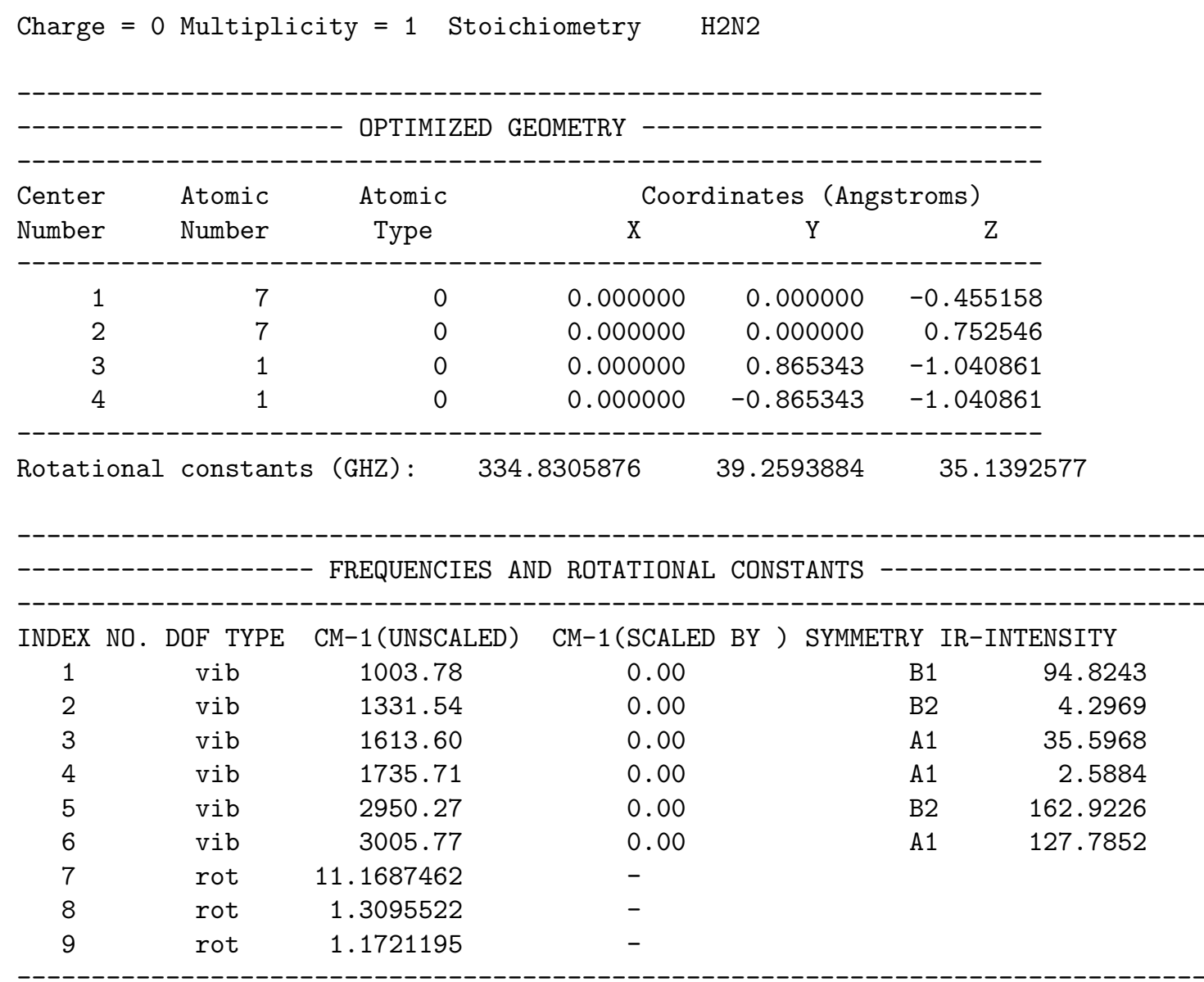

\section{Isoformyl.log}

Charge $=0$ Multiplicity $=2$ Stoichiometry $\mathrm{CHO}(2)$

\begin{tabular}{|c|c|c|c|c|c|}
\hline \multirow{2}{*}{$\begin{array}{l}\text { Center } \\
\text { Number }\end{array}$} & \multirow{2}{*}{$\begin{array}{l}\text { Atomic } \\
\text { Number }\end{array}$} & \multirow{2}{*}{$\begin{array}{l}\text { Atomic } \\
\text { Type }\end{array}$} & \multicolumn{3}{|c|}{ Coordinates (Angstroms) } \\
\hline & & & $\mathrm{X}$ & $\mathrm{Y}$ & $\mathrm{Z}$ \\
\hline 1 & 8 & 0 & 0.059774 & -0.480433 & 0.000000 \\
\hline 2 & 1 & 0 & -0.836841 & -0.893456 & 0.000000 \\
\hline 3 & 6 & 0 & 0.059774 & 0.789486 & 0.000000 \\
\hline
\end{tabular}

$\begin{array}{llll}\text { Rotational constants (GHZ) : } & 701.9597460 & 42.0774038 & 39.6978077\end{array}$

FREQUENCIES AND ROTATIONAL CONSTANTS

INDEX NO. DOF TYPE CM-1(UNSCALED) CM-1(SCALED BY ) SYMMETRY IR-INTENSITY

$\begin{array}{llllll}1 & \text { vib } & 1134.28 & 0.00 & A^{\prime} & 88.6689 \\ 2 & \text { vib } & 1388.64 & 0.00 & A^{\prime} & 34.0973\end{array}$




\begin{tabular}{|c|c|c|c|c|c|}
\hline 3 & vib & 3264.57 & 0.00 & $A^{\prime}$ & 60.7133 \\
\hline 4 & rot & 23.4148568 & - & & \\
\hline 5 & rot & 1.4035511 & - & & \\
\hline 6 & rot & 1.3241763 & - & & \\
\hline
\end{tabular}

SPIN CONTAMINATION

$\begin{array}{cccccc}\text { Charge } & \text { Multiplicity } & \mathrm{S}^{\wedge} 2 \text { Before } & \mathrm{S}^{\wedge} \text { 2 After } & \mathrm{S}^{\wedge} 2 \text { Expected } & \% \text { Difference? } \\ 0 & 2 & 0.7540 & 0.7500 & 0.7500 & 0.00\end{array}$

\section{IsofulminicAcid.log}

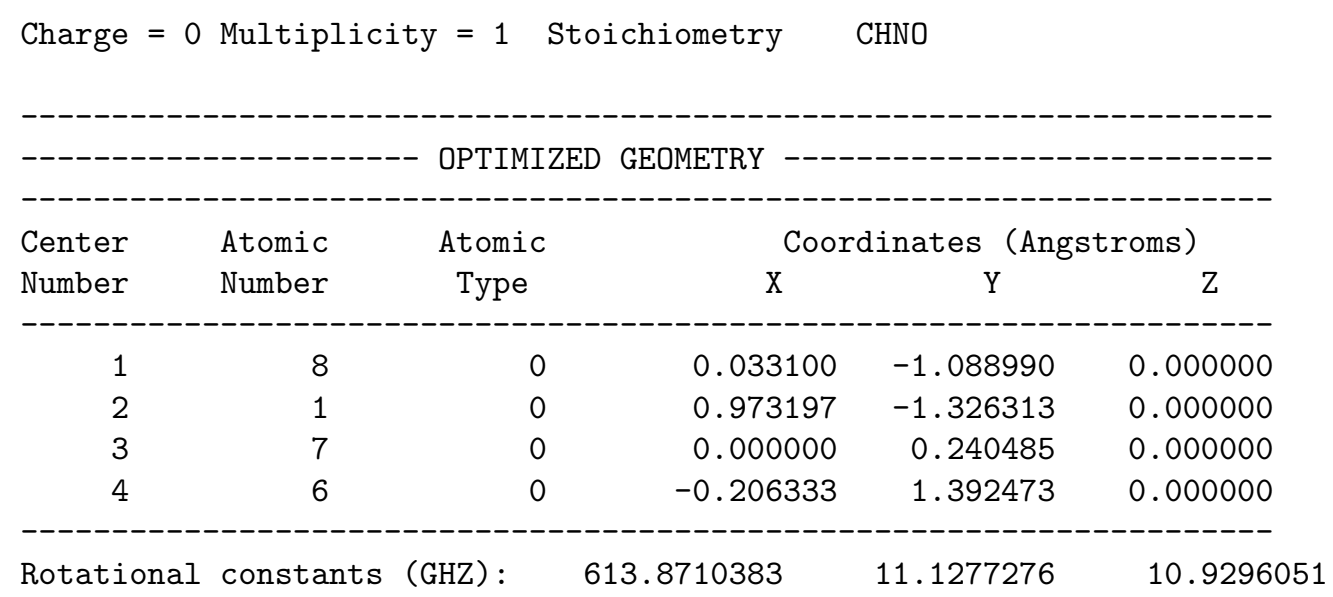

FREQUENCIES AND ROTATIONAL CONSTANTS

INDEX NO. DOF TYPE CM-1(UNSCALED) CM-1(SCALED BY) SYMMETRY IR-INTENSITY

$\begin{array}{rrrrrr}1 & \text { vib } & 264.98 & 0.00 & A^{\prime} & 4.0928 \\ 2 & \text { vib } & 322.94 & 0.00 & A^{\prime \prime} & 9.2629 \\ 3 & \text { vib } & 978.05 & 0.00 & A^{\prime} & 51.4798 \\ 4 & \text { vib } & 1415.42 & 0.00 & A^{\prime} & 61.9486 \\ 5 & \text { vib } & 2216.25 & 0.00 & A^{\prime} & 19.1674 \\ 6 & \text { vib } & 3719.26 & 0.00 & A^{\prime} & 105.4537 \\ 7 & \text { rot } & 20.4765337 & - & & \\ 8 & \text { rot } & 0.3711810 & - & & \\ 9 & \text { rot } & 0.3645724 & - & \end{array}$

\section{MethaneDiol-2.log}

Charge $=0$ Multiplicity $=1$ Stoichiometry $\quad$ CH402 


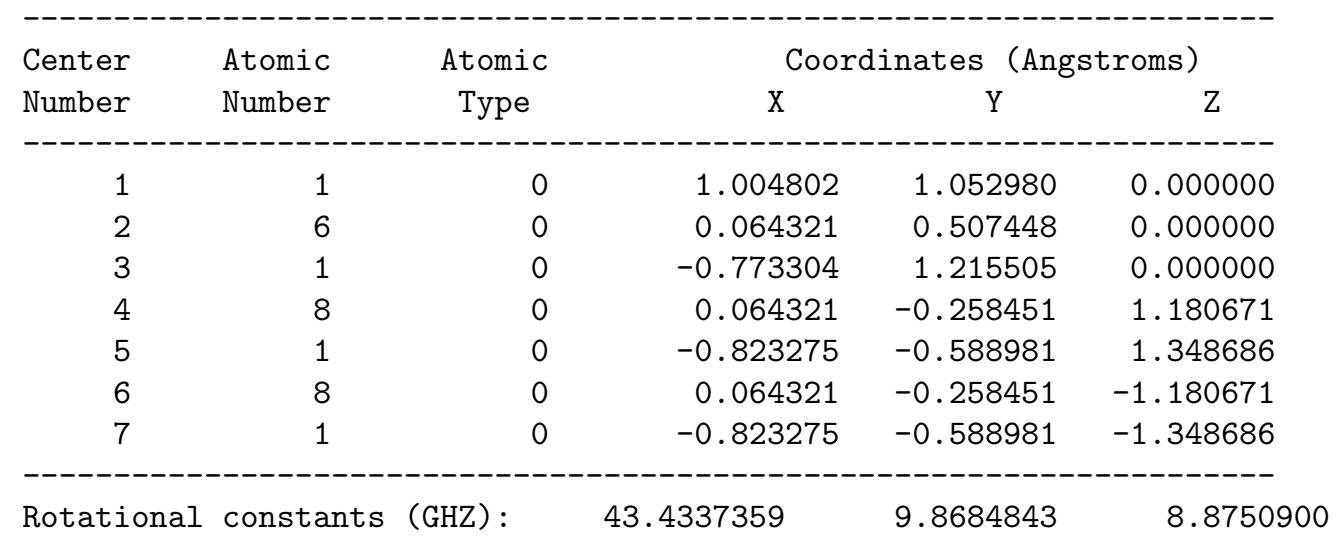

FREQUENCIES AND ROTATIONAL CONSTANTS

$\begin{array}{ccccrr}\text { INDEX NO. DOF TYPE } & \text { CM-1 (UNSCALED) } & \text { CM-1 (SCALED BY }) & \text { SYMMETRY IR-INTENSITY } \\ 1 & \text { vib } & 197.91 & 0.00 & A^{\prime \prime} & 72.1147 \\ 2 & \text { vib } & 392.34 & 0.00 & A^{\prime} & 116.5561 \\ 3 & \text { vib } & 538.23 & 0.00 & A^{\prime} & 31.0576 \\ 4 & \text { vib } & 997.26 & 0.00 & A^{\prime} & 53.3359 \\ 5 & \text { vib } & 1049.53 & 0.00 & A^{\prime \prime} & 289.9684 \\ 6 & \text { vib } & 1058.94 & 0.00 & A^{\prime} & 35.4241 \\ 7 & \text { vib } & 1168.67 & 0.00 & A^{\prime \prime} & 74.3057 \\ 8 & \text { vib } & 1375.09 & 0.00 & A^{\prime \prime} & 3.4683 \\ 9 & \text { vib } & 1413.92 & 0.00 & A^{\prime} & 21.1636 \\ 10 & \text { vib } & 1443.47 & 0.00 & A^{\prime \prime} & 27.0811 \\ 11 & \text { vib } & 1517.09 & 0.00 & A^{\prime} & 0.3578 \\ 12 & \text { vib } & 2978.44 & 0.00 & A^{\prime} & 86.2285 \\ 13 & \text { vib } & 3113.70 & 0.00 & A^{\prime} & 27.2723 \\ 14 & \text { vib } & 3812.33 & 0.00 & A^{\prime} & 32.7693 \\ 15 & \text { vib } & 3813.79 & 0.00 & & 13.5665 \\ 16 & \text { rot } & 1.4487935 & - & & \\ 17 & \text { rot } & 0.3291772 & - & & \\ 18 & \text { rot } & 0.2960411 & - & & \\ -----------------------------------------------------------------\end{array}$

\section{Methanediol.log}

Charge $=0$ Multiplicity $=1$ Stoichiometry $\quad \mathrm{CH} 402$

\begin{tabular}{|c|c|c|c|c|c|}
\hline \multirow{2}{*}{$\begin{array}{l}\text { Center } \\
\text { Number }\end{array}$} & \multirow{2}{*}{$\begin{array}{l}\text { Atomic } \\
\text { Number }\end{array}$} & \multirow{2}{*}{$\begin{array}{c}\text { Atomic } \\
\text { Type }\end{array}$} & \multicolumn{3}{|c|}{ Coordinates (Angstroms) } \\
\hline & & & $\mathrm{X}$ & Y & $\mathrm{Z}$ \\
\hline 1 & 6 & 0 & 0.000000 & 0.000000 & 0.529049 \\
\hline 2 & 1 & 0 & 0.890107 & 0.074094 & 1.157916 \\
\hline 3 & 1 & 0 & -0.890107 & -0.074094 & 1.157916 \\
\hline 4 & 8 & 0 & 0.000000 & -1.173223 & -0.246766 \\
\hline 5 & 1 & 0 & 0.808310 & -1.183792 & -0.770936 \\
\hline
\end{tabular}




\begin{tabular}{cccccc}
6 & 8 & 0 & 0.000000 & 1.173223 & -0.246766 \\
7 & 1 & 0 & -0.808310 & 1.183792 & -0.770936 \\
\hline-1 & 0 & -1.9900763 & 10.1518076 & 9.0026865
\end{tabular}

FREQUENCIES AND ROTATIONAL CONSTANTS

INDEX NO. DOF TYPE CM-1(UNSCALED) CM-1(SCALED BY ) SYMMETRY IR-INTENSITY

\begin{tabular}{|c|c|c|c|c|c|}
\hline 1 & vib & 369.77 & 0.00 & $\mathrm{~A}$ & 56.9035 \\
\hline 2 & vib & 373.57 & 0.00 & B & 166.4798 \\
\hline 3 & vib & 558.53 & 0.00 & $\mathrm{~A}$ & 63.3331 \\
\hline 4 & vib & 1010.78 & 0.00 & B & 9.3313 \\
\hline 5 & vib & 1032.33 & 0.00 & $\mathrm{~A}$ & 97.3431 \\
\hline 6 & vib & 1057.32 & 0.00 & B & 261.1010 \\
\hline 7 & vib & 1207.07 & 0.00 & $\mathrm{~A}$ & 1.4749 \\
\hline 8 & vib & 1373.68 & 0.00 & B & 34.4387 \\
\hline 9 & vib & 1393.92 & 0.00 & $\mathrm{~A}$ & 3.5218 \\
\hline 10 & vib & 1447.66 & 0.00 & B & 55.6139 \\
\hline 11 & vib & 1530.78 & 0.00 & $\mathrm{~A}$ & 0.4105 \\
\hline 12 & vib & 3022.22 & 0.00 & $\mathrm{~A}$ & 62.9983 \\
\hline 13 & vib & 3066.30 & 0.00 & B & 50.6113 \\
\hline 14 & vib & 3796.62 & 0.00 & B & 36.9051 \\
\hline 15 & vib & 3797.29 & 0.00 & $\mathrm{~A}$ & 20.6935 \\
\hline 16 & rot & 1.4006382 & - & & \\
\hline 17 & rot & 0.3386279 & - & & \\
\hline 18 & rot & 0.3002973 & - & & \\
\hline
\end{tabular}

\section{MethaneDiols-freqs.log}

Charge $=0$ Multiplicity $=1$ Stoichiometry $\quad$ CH402

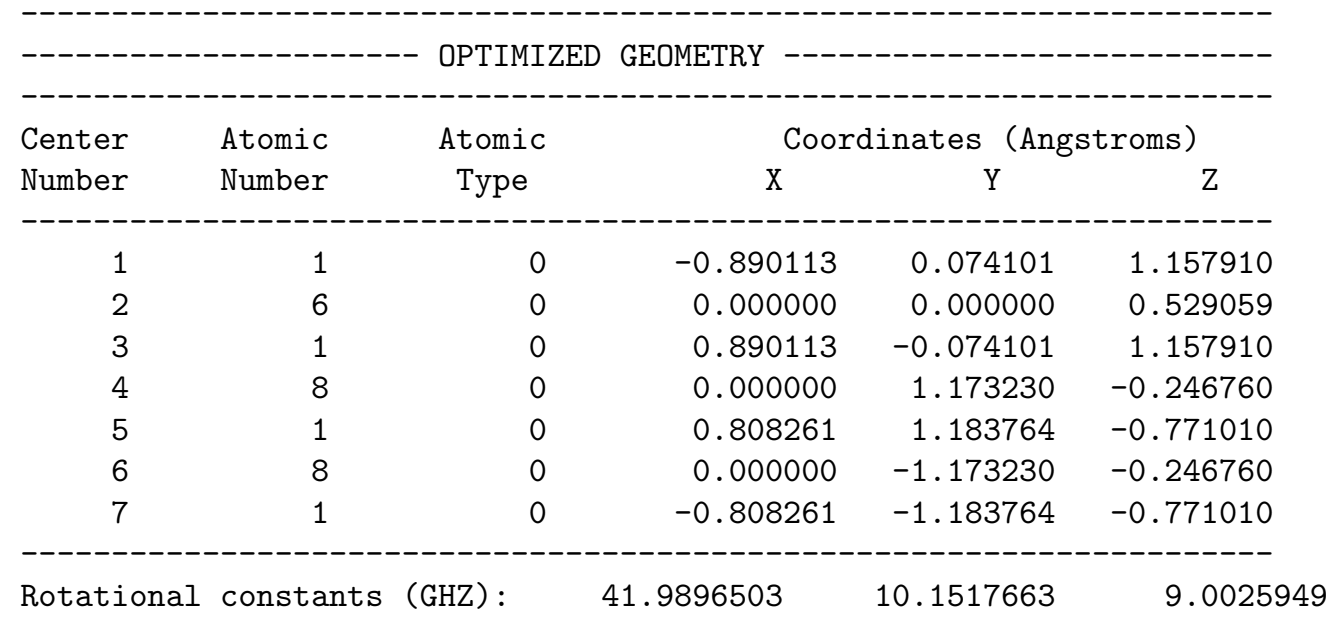

FREQUENCIES AND ROTATIONAL CONSTANTS

INDEX NO. DOF TYPE CM-1(UNSCALED) CM-1(SCALED BY) SYMMETRY IR-INTENSITY 


\begin{tabular}{|c|c|c|c|c|c|}
\hline 1 & vib & 374.40 & 0.00 & A & 55.7376 \\
\hline 2 & vib & 377.05 & 0.00 & B & 166.4464 \\
\hline 3 & vib & 559.38 & 0.00 & A & 64.5316 \\
\hline 4 & vib & 1011.26 & 0.00 & B & 8.9945 \\
\hline 5 & vib & 1032.54 & 0.00 & $\mathrm{~A}$ & 97.2101 \\
\hline 6 & vib & 1057.36 & 0.00 & B & 261.2220 \\
\hline 7 & vib & 1207.76 & 0.00 & A & 1.5069 \\
\hline 8 & vib & 1374.04 & 0.00 & B & 34.3723 \\
\hline 9 & vib & 1394.43 & 0.00 & A & 3.5597 \\
\hline 10 & vib & 1447.82 & 0.00 & B & 55.9973 \\
\hline 11 & vib & 1530.49 & 0.00 & A & 0.4147 \\
\hline 12 & vib & 3021.58 & 0.00 & A & 62.9890 \\
\hline 13 & vib & 3065.67 & 0.00 & B & 50.6100 \\
\hline 14 & vib & 3796.38 & 0.00 & B & 36.8828 \\
\hline 15 & vib & 3797.07 & 0.00 & A & 20.7037 \\
\hline 16 & rot & 1.4006240 & - & & \\
\hline 17 & rot & 0.3386265 & - & & \\
\hline 18 & rot & 0.3002942 & - & & \\
\hline
\end{tabular}

2) LEVEL OF THEORY: B3LYP/CC-PVTZ+D

Charge $=0$ Multiplicity $=1$ Stoichiometry $\quad \mathrm{CH} 402$

\begin{tabular}{|c|c|c|c|c|c|}
\hline \multirow{2}{*}{$\begin{array}{l}\text { Center } \\
\text { Number }\end{array}$} & \multirow{2}{*}{$\begin{array}{l}\text { Atomic } \\
\text { Number }\end{array}$} & \multirow{2}{*}{$\begin{array}{c}\text { Atomic } \\
\text { Type }\end{array}$} & \multicolumn{3}{|c|}{ Coordinates (Angstroms) } \\
\hline & & & $\mathrm{X}$ & $\mathrm{Y}$ & $\mathrm{Z}$ \\
\hline 1 & 1 & 0 & 1.004802 & 1.052980 & 0.000000 \\
\hline 2 & 6 & 0 & 0.064321 & 0.507448 & 0.000000 \\
\hline 3 & 1 & 0 & -0.773304 & 1.215505 & 0.000000 \\
\hline 4 & 8 & 0 & 0.064321 & -0.258451 & 1.180671 \\
\hline 5 & 1 & 0 & -0.823275 & -0.588981 & 1.348686 \\
\hline 6 & 8 & 0 & 0.064321 & -0.258451 & -1.180671 \\
\hline 7 & 1 & 0 & -0.823275 & -0.588981 & -1.348686 \\
\hline Rotatio & constan & (14) & 4337359 & 9.8684843 & 8.875090 \\
\hline
\end{tabular}

FREQUENCIES AND ROTATIONAL CONSTANTS

INDEX NO. DOF TYPE CM-1(UNSCALED) CM-1(SCALED BY) SYMMETRY IR-INTENSITY

$\begin{array}{rrrrrr}1 & \text { vib } & 197.91 & 0.00 & \text { A" } & 72.1147 \\ 2 & \text { vib } & 392.34 & 0.00 & \text { A', } & 116.5561 \\ 3 & \text { vib } & 538.23 & 0.00 & \text { A', } & 31.0576 \\ 4 & \text { vib } & 997.26 & 0.00 & A^{\prime} & 53.3359 \\ 5 & \text { vib } & 1049.53 & 0.00 & A^{\prime \prime} & 289.9684 \\ 6 & \text { vib } & 1058.94 & 0.00 & A^{\prime} & 35.4241 \\ 7 & \text { vib } & 1168.67 & 0.00 & A^{\prime \prime} & 74.3057 \\ 8 & \text { vib } & 1375.09 & 0.00 & A^{\prime \prime} & 3.4683 \\ 9 & \text { vib } & 1413.92 & 0.00 & A^{\prime} & 21.1636 \\ 10 & \text { vib } & 1443.47 & 0.00 & \text { A" } & 27.0811 \\ 11 & \text { vib } & 1517.09 & 0.00 & \text { A' } & 0.3578\end{array}$




\begin{tabular}{llllll}
12 & vib & 2978.44 & 0.00 & $A^{\prime}$ & 86.2285 \\
13 & vib & 3113.70 & 0.00 & $A^{\prime}$ & 27.2723 \\
14 & vib & 3812.33 & 0.00 & $A^{\prime}$ & 32.7693 \\
15 & vib & 3813.79 & 0.00 & $A^{\prime \prime}$ & 13.5665 \\
16 & rot & 1.4487935 & - & \\
17 & rot & 0.3291772 & - & \\
18 & rot & 0.2960411 & - & \\
\hline
\end{tabular}

3) LEVEL OF THEORY: B3LYP/CC-PVTZ+D

Charge $=0$ Multiplicity $=1$ Stoichiometry $\quad \mathrm{CH} 402$

\begin{tabular}{|c|c|c|c|c|c|}
\hline \multirow{2}{*}{$\begin{array}{l}\text { Center } \\
\text { Number }\end{array}$} & \multirow{2}{*}{$\begin{array}{l}\text { Atomic } \\
\text { Number }\end{array}$} & \multirow{2}{*}{$\begin{array}{c}\text { Atomic } \\
\text { Type }\end{array}$} & \multicolumn{3}{|c|}{ Coordinates (Angstroms) } \\
\hline & & & $\mathrm{X}$ & $\mathrm{Y}$ & Z \\
\hline 1 & 1 & 0 & 1.004802 & 1.052980 & 0.000000 \\
\hline 2 & 6 & 0 & 0.064321 & 0.507448 & 0.000000 \\
\hline 3 & 1 & 0 & -0.773304 & 1.215505 & 0.000000 \\
\hline 4 & 8 & 0 & 0.064321 & -0.258451 & 1.180670 \\
\hline 5 & 1 & 0 & -0.823275 & -0.588981 & 1.348686 \\
\hline 6 & 8 & 0 & 0.064321 & -0.258451 & -1.180670 \\
\hline 7 & 1 & 0 & -0.823275 & -0.588981 & -1.348686 \\
\hline
\end{tabular}

FREQUENCIES AND ROTATIONAL CONSTANTS

INDEX NO. DOF TYPE CM-1(UNSCALED) CM-1(SCALED BY ) SYMMETRY IR-INTENSITY

$\begin{array}{lrrrr}\text { vib } & 197.90 & 0.00 & A^{\prime \prime} & 72.1154 \\ \text { vib } & 392.34 & 0.00 & A^{\prime} & 116.5581 \\ \text { vib } & 538.23 & 0.00 & A^{\prime} & 31.0565 \\ \text { vib } & 997.26 & 0.00 & A^{\prime} & 53.3361 \\ \text { vib } & 1049.53 & 0.00 & A^{\prime \prime} & 289.9690 \\ \text { vib } & 1058.94 & 0.00 & A^{\prime} & 35.4239 \\ \text { vib } & 1168.67 & 0.00 & A^{\prime \prime} & 74.3056 \\ \text { vib } & 1375.09 & 0.00 & A^{\prime \prime} & 3.4681 \\ \text { vib } & 1413.92 & 0.00 & A^{\prime} & 21.1634 \\ \text { vib } & 1443.47 & 0.00 & A^{\prime \prime} & 27.0811 \\ \text { vib } & 1517.09 & 0.00 & A^{\prime} & 0.3578 \\ \text { vib } & 2978.44 & 0.00 & A^{\prime} & 86.2288 \\ \text { vib } & 3113.70 & 0.00 & A^{\prime} & 27.2725 \\ \text { vib } & 3812.33 & 0.00 & A^{\prime \prime} & 13.5663 \\ \text { vib } & 3813.79 & 0.00 & & \\ \text { rot } & 1.4487929 & - & & \\ \text { rot } & 0.3291773 & - & & \end{array}$


4) LEVEL OF THEORY: B3LYP/CC-PVTZ+D

Charge $=0$ Multiplicity $=1$ Stoichiometry $\quad$ CH402

\begin{tabular}{|c|c|c|c|c|c|}
\hline \multirow{2}{*}{$\begin{array}{l}\text { Center } \\
\text { Number }\end{array}$} & \multirow{2}{*}{$\begin{array}{l}\text { Atomic } \\
\text { Number }\end{array}$} & \multirow{2}{*}{$\begin{array}{c}\text { Atomic } \\
\text { Type }\end{array}$} & \multicolumn{3}{|c|}{ Coordinates (Angstroms) } \\
\hline & & & $\mathrm{X}$ & $\mathrm{Y}$ & $\mathrm{Z}$ \\
\hline 1 & 1 & 0 & -0.000001 & 0.920600 & 1.108124 \\
\hline 2 & 6 & 0 & 0.000000 & 0.502789 & 0.094026 \\
\hline 3 & 1 & 0 & 0.000000 & 1.309920 & -0.634431 \\
\hline 4 & 8 & 0 & -1.180669 & -0.226379 & -0.140316 \\
\hline 5 & 1 & 0 & -1.348714 & -0.812595 & 0.603604 \\
\hline 6 & 8 & 0 & 1.180669 & -0.226379 & -0.140316 \\
\hline 7 & 1 & 0 & 1.348715 & -0.812595 & 0.603604 \\
\hline
\end{tabular}

FREQUENCIES AND ROTATIONAL CONSTANTS

INDEX NO. DOF TYPE CM-1(UNSCALED) CM-1(SCALED BY) SYMMETRY IR-INTENSITY

$\begin{array}{lllll}\text { vib } & 198.42 & 0.00 & \text { A } & 72.1130\end{array}$

vib $\quad 392.56 \quad 0.00$

A $\quad 116.5275$

$2 \quad$ vib

0.00

vib

997.31

0.00

31.0842

vib

1049.54

0.00

53.3279

vib

1058.94

0.00

289.9138

vib

1168.79

0.00

35.4242

vib

1375.12

0.00

74.3349

vib

1414.00

0.00

1443.44

0.00

3.4816

vib

1517.07

0.00

21.1720

vib

2978.39

0.00

27.0857

0.3577

vib

3113.68

0.00

86.2300

vib

3812.46

0.00

27.2719

3813.93

0.00

32.7700

vib

1.4487989

$-$

rot $\quad 0.3291768$

rot

0.2960416

\section{Methylamidogen.log}

Charge $=0$ Multiplicity $=2$ Stoichiometry $\quad$ CH4N(2)

$\begin{array}{llcl}\text { Center } & \text { Atomic } & \text { Atomic } & \text { OPTIMIZED GEOMETRY } \\ \text { Number } & \text { Number } & \text { Type } & \text { X Coordinates (Angstroms) } \\ \text { Z }\end{array}$




\begin{tabular}{rrrrrr}
1 & 6 & 0 & -0.059803 & -0.623968 & 0.000000 \\
2 & 1 & 0 & 0.467537 & -1.028618 & 0.876215 \\
3 & 1 & 0 & 0.467537 & -1.028618 & -0.876215 \\
4 & 1 & 0 & -1.079302 & -1.008504 & 0.000000 \\
5 & 7 & 0 & -0.059803 & 0.814147 & 0.000000 \\
6 & 1 & 0 & 0.921670 & 1.110518 & 0.000000 \\
Rotational constants (GHZ) : & \multicolumn{1}{c}{125.3716072} & 25.4770091 & 24.3290256
\end{tabular}

FREQUENCIES AND ROTATIONAL CONSTANTS

INDEX NO. DOF TYPE CM-1(UNSCALED) CM-1(SCALED BY) SYMMETRY IR-INTENSITY

\begin{tabular}{|c|c|c|c|c|c|}
\hline 1 & vib & 249.99 & 0.00 & $A^{\prime \prime}$ & 51.8763 \\
\hline 2 & vib & 948.27 & 0.00 & $A^{\prime \prime}$ & 0.2701 \\
\hline 3 & vib & 1006.26 & 0.00 & $A^{\prime}$ & 12.9473 \\
\hline 4 & vib & 1041.31 & 0.00 & $A^{\prime}$ & 16.6584 \\
\hline 5 & vib & 1338.57 & 0.00 & $A^{\prime}$ & 10.0751 \\
\hline 6 & vib & 1392.43 & 0.00 & $A^{\prime}$ & 12.5826 \\
\hline 7 & vib & 1476.10 & 0.00 & $A^{\prime \prime}$ & 6.9745 \\
\hline 8 & vib & 1485.13 & 0.00 & $A^{\prime}$ & 3.4746 \\
\hline 9 & vib & 2944.55 & 0.00 & $A^{\prime}$ & 15.8059 \\
\hline 10 & vib & 2974.64 & 0.00 & A" & 28.3419 \\
\hline 11 & vib & 3083.19 & 0.00 & $A^{\prime}$ & 30.7793 \\
\hline 12 & vib & 3378.63 & 0.00 & $A^{\prime}$ & 8.8578 \\
\hline 13 & rot & 4.1819467 & - & & \\
\hline 14 & rot & 0.8498215 & - & & \\
\hline 15 & rot & 0.8115289 & - & & \\
\hline
\end{tabular}

SPIN CONTAMINATION

$\begin{array}{cccccc}\text { Charge } & \text { Multiplicity } & \mathrm{S}^{\wedge} 2 \text { Before } & \mathrm{S}^{\wedge} 2 \text { After } & \mathrm{S}^{\wedge} 2 \text { Expected } & \% \text { Difference? } \\ 0 & 2 & 0.7537 & 0.7500 & 0.7500 & 0.00\end{array}$

\section{Methylenimine.log}

Charge $=0$ Multiplicity $=1$ Stoichiometry $\quad$ CH3N

\begin{tabular}{|c|c|c|c|c|c|}
\hline \multirow{2}{*}{$\begin{array}{l}\text { Center } \\
\text { Number }\end{array}$} & \multirow{2}{*}{$\begin{array}{l}\text { Atomic } \\
\text { Number }\end{array}$} & \multirow{2}{*}{$\begin{array}{c}\text { Atomic } \\
\text { Type }\end{array}$} & \multicolumn{3}{|c|}{ Coordinates (Angstroms) } \\
\hline & & & $\mathrm{X}$ & $\mathrm{Y}$ & $\mathrm{Z}$ \\
\hline 1 & 6 & 0 & 0.055966 & 0.582356 & 0.000000 \\
\hline 2 & 7 & 0 & 0.055966 & -0.680862 & 0.000000 \\
\hline 3 & 1 & 0 & -0.840320 & 1.209778 & 0.000000 \\
\hline 4 & 1 & 0 & 1.009799 & 1.109425 & 0.000000 \\
\hline 5 & 1 & 0 & -0.897038 & -1.047308 & 0.000000 \\
\hline
\end{tabular}




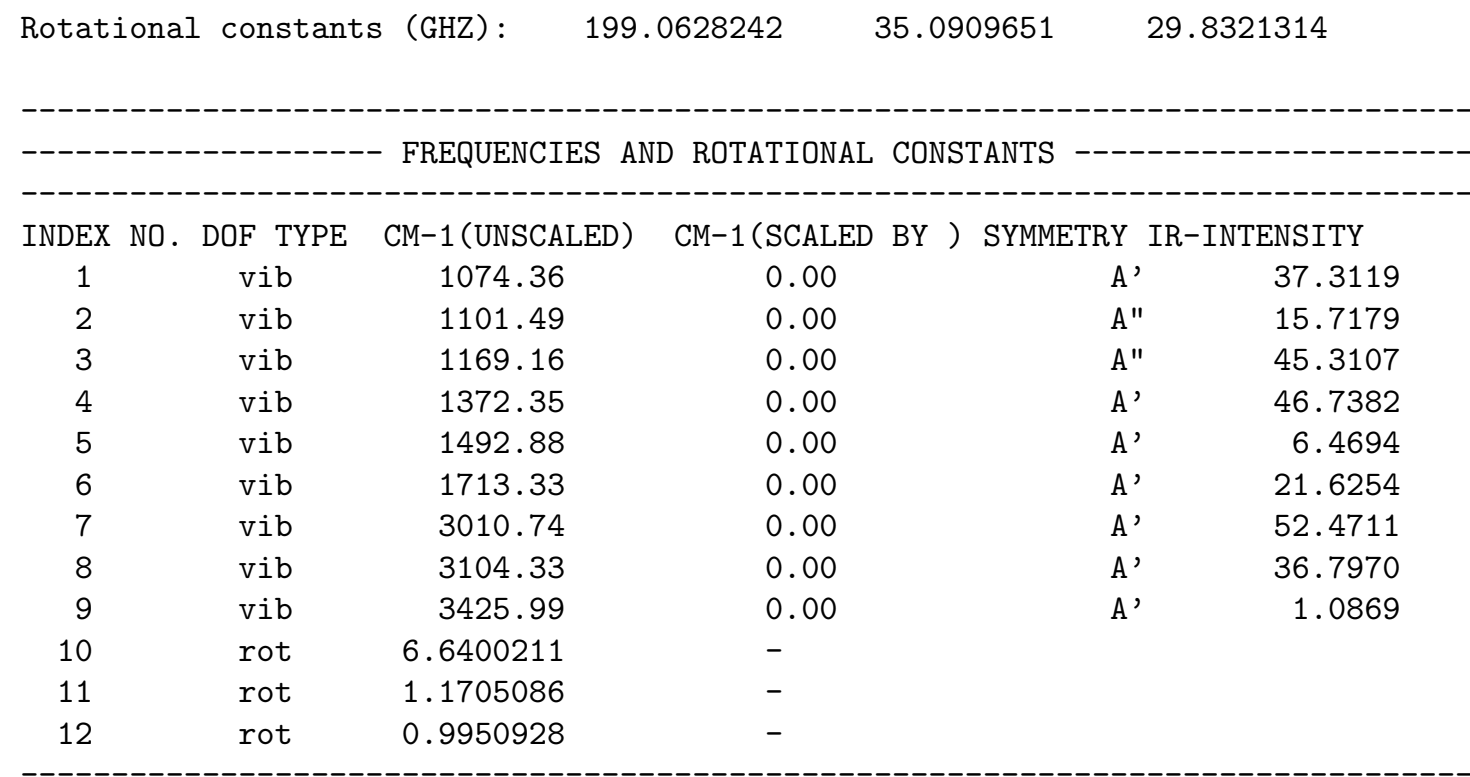

\section{Methylhydroperoxide.log}

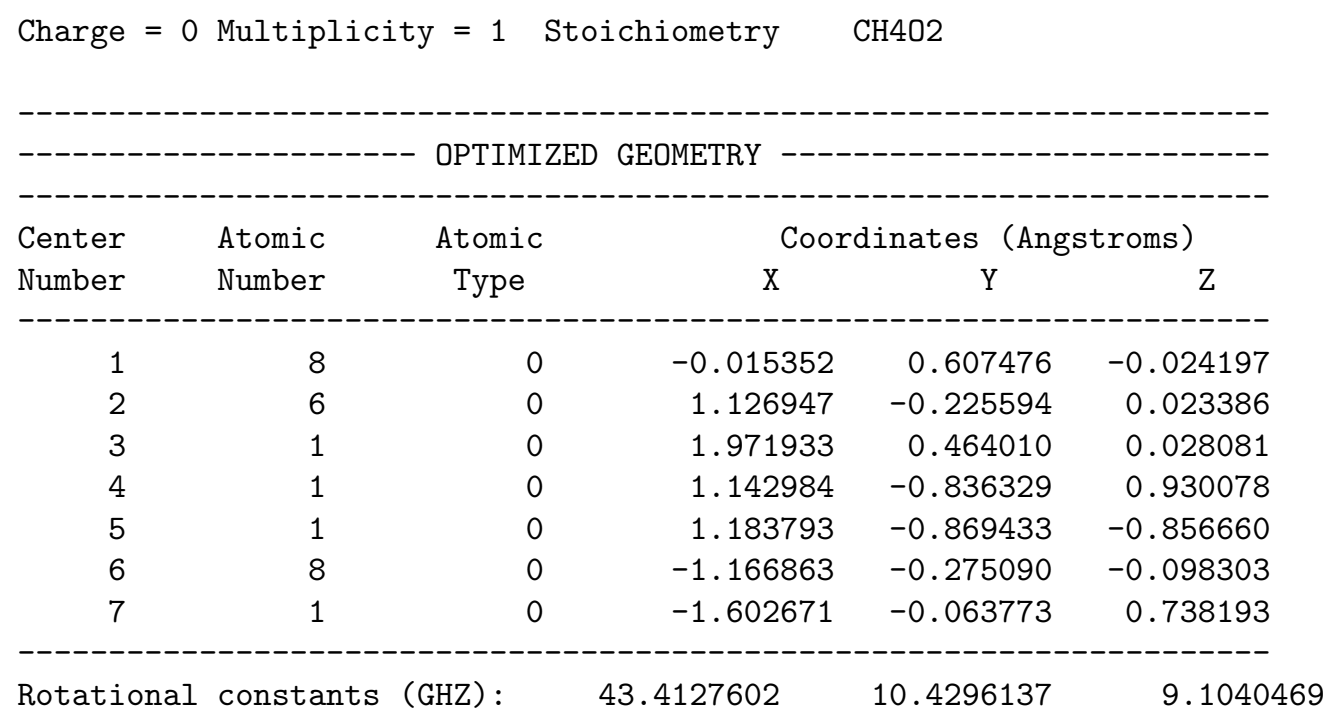

FREQUENCIES AND ROTATIONAL CONSTANTS

INDEX NO. DOF TYPE CM-1(UNSCALED) CM-1 (SCALED BY ) SYMMETRY IR-INTENSITY

\begin{tabular}{|c|c|c|c|c|c|}
\hline 1 & vib & 195.47 & 0.00 & A & 107.3107 \\
\hline 2 & vib & 249.67 & 0.00 & A & 11.1008 \\
\hline 3 & vib & 446.95 & 0.00 & A & 6.4389 \\
\hline 4 & vib & 884.83 & 0.00 & A & 17.8894 \\
\hline 5 & vib & 1037.51 & 0.00 & A & 17.3407 \\
\hline 6 & vib & 1172.65 & 0.00 & A & 2.3131 \\
\hline 7 & vib & 1207.48 & 0.00 & $\mathrm{~A}$ & 5.6981 \\
\hline 8 & vib & 1370.08 & 0.00 & A & 48.9431 \\
\hline 9 & vib & 1449.94 & 0.00 & A & 1.2577 \\
\hline
\end{tabular}




$\begin{array}{lllllr}10 & \text { vib } & 1462.20 & 0.00 & \text { A } & 5.9949 \\ 11 & \text { vib } & 1512.12 & 0.00 & \text { A } & 9.4502 \\ 12 & \text { vib } & 3004.35 & 0.00 & \text { A } & 40.0400 \\ 13 & \text { vib } & 3068.28 & 0.00 & \text { A } & 46.2630 \\ 14 & \text { vib } & 3102.48 & 0.00 & \text { A } & 17.1433 \\ 15 & \text { vib } & 3760.56 & 0.00 & \text { A } & 34.8295 \\ 16 & \text { rot } & 1.4480938 & - & & \\ 17 & \text { rot } & 0.3478945 & - & & \\ 18 & \text { rot } & 0.3036783 & - & \end{array}$

\section{Methylperoxy.log}

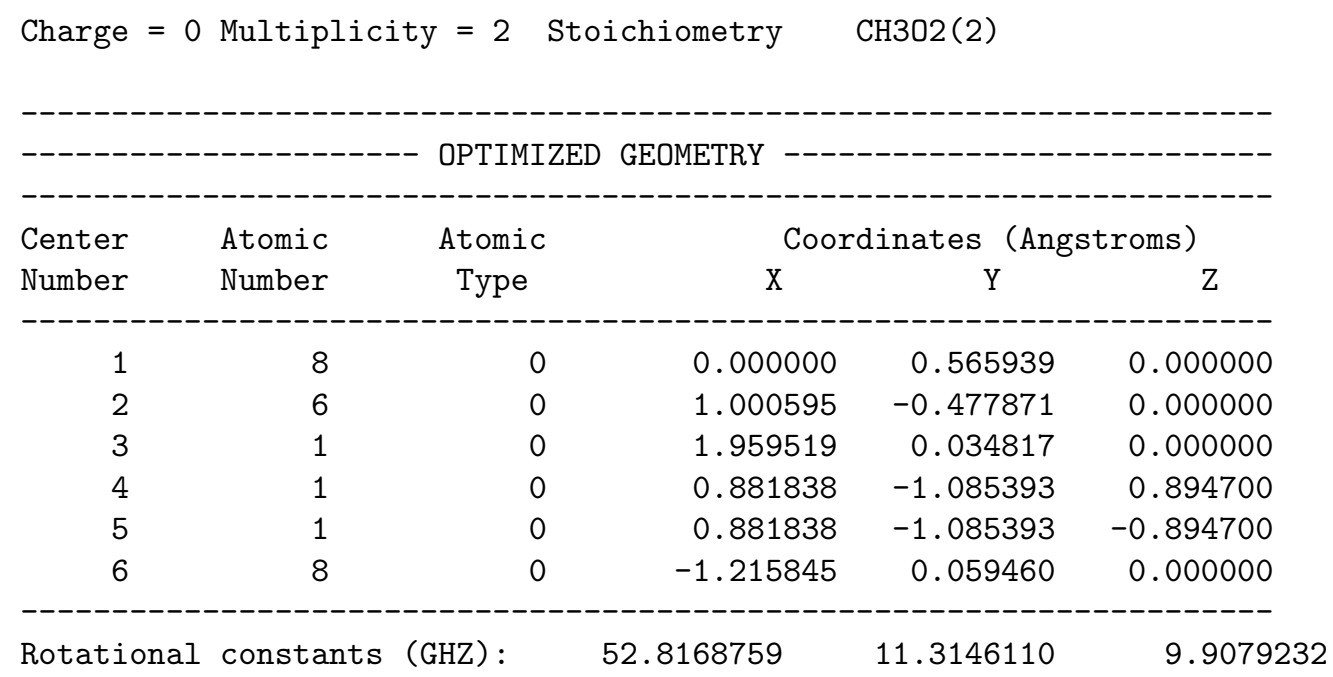

FREQUENCIES AND ROTATIONAL CONSTANTS

INDEX NO. DOF TYPE CM-1(UNSCALED) CM-1(SCALED BY) SYMMETRY IR-INTENSITY

\begin{tabular}{|c|c|c|c|c|c|}
\hline 1 & vib & 127.77 & 0.00 & $A^{\prime \prime}$ & 0.0885 \\
\hline 2 & vib & 491.55 & 0.00 & $A^{\prime}$ & 6.5800 \\
\hline 3 & vib & 915.17 & 0.00 & $A^{\prime}$ & 13.2232 \\
\hline 4 & vib & 1129.18 & 0.00 & $A^{\prime \prime}$ & 0.7003 \\
\hline 5 & vib & 1154.41 & 0.00 & $A^{\prime}$ & 1.8988 \\
\hline 6 & vib & 1217.11 & 0.00 & $A^{\prime}$ & 9.9712 \\
\hline 7 & vib & 1443.14 & 0.00 & $A^{\prime}$ & 1.1860 \\
\hline 8 & vib & 1472.65 & 0.00 & $A^{\prime \prime}$ & 9.1425 \\
\hline 9 & vib & 1484.60 & 0.00 & $A^{\prime}$ & 8.5912 \\
\hline 10 & vib & 3048.04 & 0.00 & $A^{\prime}$ & 14.4839 \\
\hline 11 & vib & 3135.47 & 0.00 & $A^{\prime \prime}$ & 12.9970 \\
\hline 12 & vib & 3148.20 & 0.00 & $A^{\prime}$ & 9.4940 \\
\hline 13 & rot & 1.7617813 & - & & \\
\hline 14 & rot & 0.3774148 & - & & \\
\hline 15 & rot & 0.3304927 & - & & \\
\hline
\end{tabular}




\begin{tabular}{|c|c|c|c|c|c|}
\hline $\begin{array}{c}\text { Charge } \\
0\end{array}$ & $\begin{array}{c}\text { Multiplicity } \\
2\end{array}$ & $\begin{array}{c}\text { S^2 Before } \\
0.7537\end{array}$ & $\begin{array}{l}\mathrm{S}^{\wedge} 2 \text { After } \\
0.7500\end{array}$ & $\begin{array}{c}\mathrm{S}^{\wedge} 2 \text { Expected } \\
0.7500\end{array}$ & $\begin{array}{c}\% \text { Difference? } \\
0.00\end{array}$ \\
\hline
\end{tabular}

\section{$92 \quad \mathrm{~N} 2 \mathrm{H} 3 . \log$}

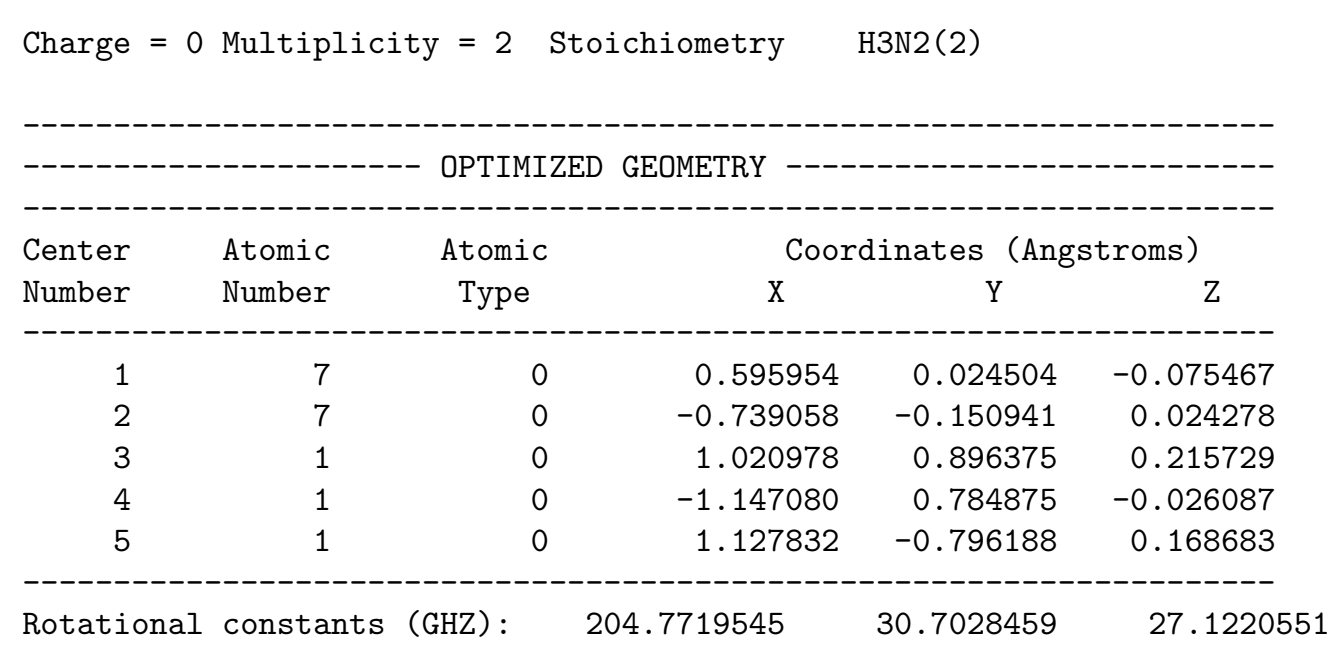

FREQUENCIES AND ROTATIONAL CONSTANTS

$\begin{array}{ccccrr}\text { INDEX NO. DOF TYPE } & \text { CM-1(UNSCALED) } & \text { CM-1(SCALED BY } & \text { 1) } & \text { SYMMETRY } & \text { IR-INTENSITY } \\ 1 & \text { vib } & 587.87 & 587.87 & \mathrm{~A} & 111.3632 \\ 2 & \text { vib } & 704.33 & 704.33 & \mathrm{~A} & 132.1567 \\ 3 & \text { vib } & 1141.19 & 1141.19 & \mathrm{~A} & 21.2230 \\ 4 & \text { vib } & 1228.77 & 1228.77 & \mathrm{~A} & 3.3791 \\ 5 & \text { vib } & 1487.01 & 1487.01 & \mathrm{~A} & 22.3699 \\ 6 & \text { vib } & 1656.95 & 1656.95 & \mathrm{~A} & 14.3832 \\ 7 & \text { vib } & 3406.65 & 3406.65 & \mathrm{~A} & 8.2614 \\ 8 & \text { vib } & 3471.82 & 3471.82 & \mathrm{~A} & 0.7130 \\ 9 & \text { vib } & 3619.37 & 3619.37 & \mathrm{~A} & 14.3408 \\ 10 & \text { rot } & 6.8304572 & - & & \\ 11 & \text { rot } & 1.0241367 & - & & \\ 12 & \text { rot } & 0.9046944 & - & & \\ -\end{array}$

\begin{tabular}{|c|c|c|c|c|c|}
\hline Charge & Multiplicity & $\mathrm{S}^{\wedge} 2$ Before & $S^{\wedge} 2$ After & $S^{\wedge} 2$ Expected & $\%$ Difference? \\
\hline 0 & 2 & 0.7540 & 0.7500 & 0.7500 & 0.00 \\
\hline
\end{tabular}

\section{NCCN-freqs.log}

Charge $=0$ Multiplicity $=1 \quad$ Stoichiometry $\quad$ C2N2 


\begin{tabular}{|c|c|c|c|c|c|c|}
\hline \multicolumn{7}{|c|}{ Center Atomic $\quad$ Atomic $\quad$ Coordinates (Angstroms) } \\
\hline Number & Number & Type & $\mathrm{X}$ & $\mathrm{Y}$ & \multicolumn{2}{|r|}{$\mathrm{Z}$} \\
\hline 1 & 6 & 0 & 0.000000 & 0.000000 & \multicolumn{2}{|c|}{0.687597} \\
\hline 2 & 7 & 0 & 0.000000 & 0.000000 & \multicolumn{2}{|c|}{1.840107} \\
\hline 3 & 6 & 0 & 0.000000 & 0.000000 & \multicolumn{2}{|c|}{-0.687597} \\
\hline 4 & 7 & 0 & 0.000000 & 0.000000 & \multicolumn{2}{|c|}{-1.840107} \\
\hline \multicolumn{3}{|c|}{ Rotational constants (GHZ): } & 0000000 & 4.7598428 & \multicolumn{2}{|c|}{4.7598428} \\
\hline \multicolumn{7}{|c|}{ ( } \\
\hline \multicolumn{7}{|c|}{ INDEX NO. DOF TYPE CM-1 (UNSCALED) CM-1 (SCALED BY ) SYMMETRY IR-INTENSITY } \\
\hline 1 & vib & 253.38 & 0.00 & & PIU & 21.1860 \\
\hline 2 & vib & 253.38 & 0.00 & & PIU & 21.1860 \\
\hline 3 & vib & 550.83 & 0.00 & & PIG & 0.0000 \\
\hline 4 & vib & 550.83 & 0.00 & & PIG & 0.0000 \\
\hline 5 & vib & 884.57 & 0.00 & & SGG & 0.0000 \\
\hline 6 & vib & 2267.23 & 0.00 & & SGU & 0.0059 \\
\hline 7 & vib & 2433.95 & 0.00 & & SGG & 0.0000 \\
\hline 8 & vib & 0.00 & 0.00 & & & \\
\hline 9 & vib & 0.00 & 0.00 & & & \\
\hline 10 & rot & 0.0000000 & - & & & \\
\hline 11 & rot & 0.1587713 & - & & & \\
\hline 12 & rot & 0.1587713 & - & & & \\
\hline
\end{tabular}

\section{NCN-freqs.log}

Charge $=0$ Multiplicity $=3$ Stoichiometry CN2(3)

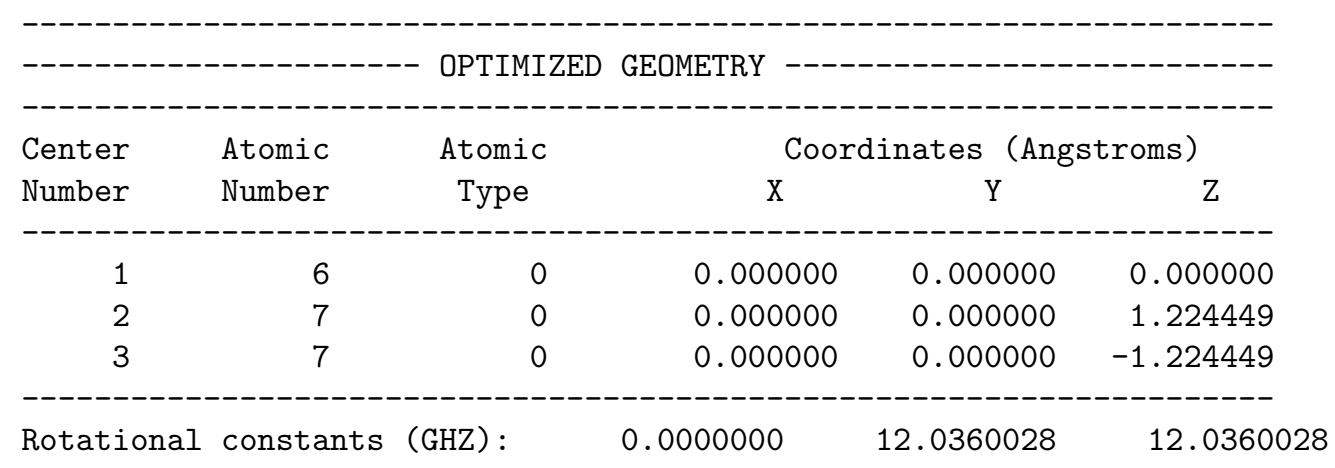

FREQUENCIES AND ROTATIONAL CONSTANTS

INDEX NO. DOF TYPE CM-1(UNSCALED) CM-1(SCALED BY) SYMMETRY IR-INTENSITY

$\begin{array}{llllll}1 & \text { Vib } & 448.37 & 0.00 & \text { PIU } & 19.8742\end{array}$ 


\begin{tabular}{rrrlrr}
2 & vib & 448.37 & 0.00 & PIU & 19.8742 \\
3 & vib & 1273.96 & 0.00 & SGG & 0.0000 \\
4 & vib & 1560.62 & 0.00 & SGU & 198.3510 \\
5 & vib & 0.00 & 0.00 & & \\
6 & vib & 0.00 & 0.00 & & \\
7 & rot & 0.0000000 & - & & \\
8 & rot & 0.4014778 & - & & \\
9 & rot & 0.4014778 & - & & \\
\hline
\end{tabular}

\section{NitricAcid.log}

Charge $=0$ Multiplicity $=1$ Stoichiometry HNO3

\begin{tabular}{|c|c|c|c|c|c|}
\hline \multirow{2}{*}{$\begin{array}{l}\text { Center } \\
\text { Number }\end{array}$} & \multirow{2}{*}{$\begin{array}{l}\text { Atomic } \\
\text { Number }\end{array}$} & \multirow{2}{*}{$\begin{array}{c}\text { Atomic } \\
\text { Type }\end{array}$} & \multicolumn{3}{|c|}{ Coordinates (Angstroms) } \\
\hline & & & $\mathrm{X}$ & Y & $\mathrm{Z}$ \\
\hline 1 & 7 & 0 & 0.000000 & 0.155821 & 0.000000 \\
\hline 2 & 8 & 0 & -0.267338 & -1.231624 & 0.000000 \\
\hline 3 & 8 & 0 & 1.168139 & 0.461319 & 0.000000 \\
\hline 4 & 8 & 0 & -0.978515 & 0.836759 & 0.000000 \\
\hline 5 & 1 & 0 & 0.621709 & -1.622386 & 0.000000 \\
\hline
\end{tabular}

FREQUENCIES AND ROTATIONAL CONSTANTS

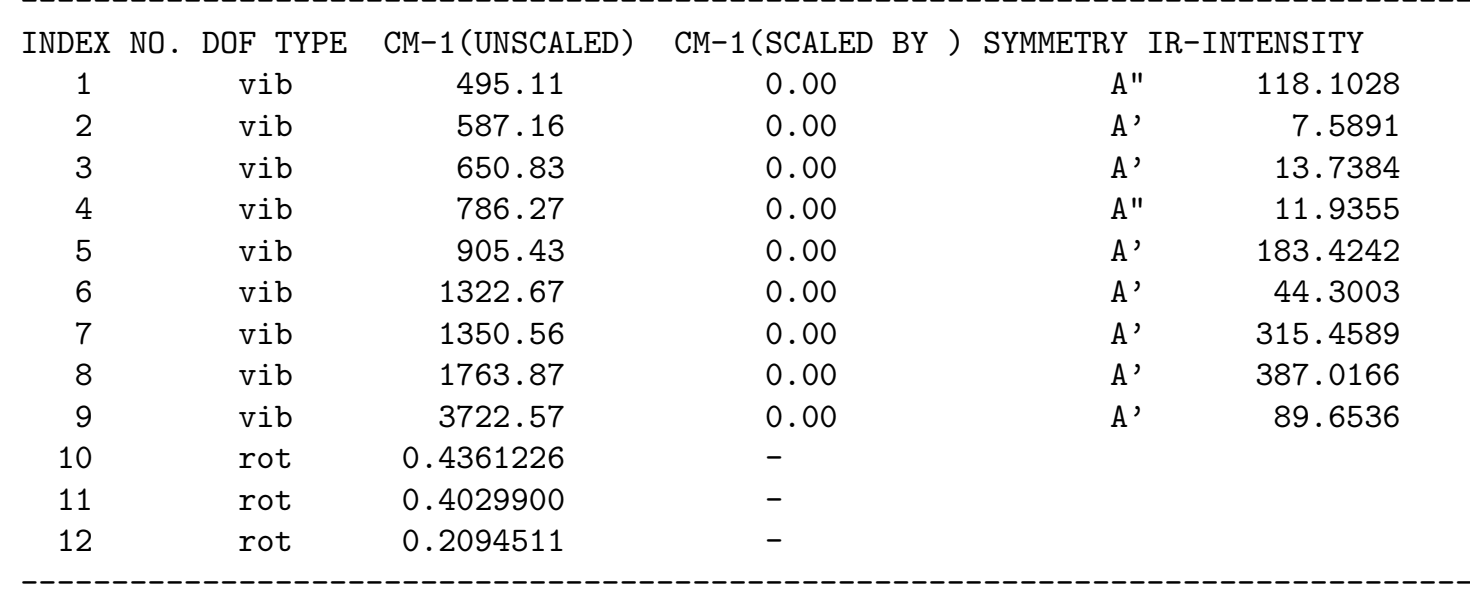




\section{Nitrosobenzene.log}

\begin{tabular}{|c|c|c|c|c|c|}
\hline \multirow{2}{*}{$\begin{array}{l}\text { Center } \\
\text { Number }\end{array}$} & \multirow{2}{*}{$\begin{array}{l}\text { Atomic } \\
\text { Number }\end{array}$} & \multirow{2}{*}{$\begin{array}{c}\text { Atomic } \\
\text { Type }\end{array}$} & \multicolumn{3}{|c|}{ Coordinates (Angstroms) } \\
\hline & & & $\mathrm{X}$ & $\mathrm{Y}$ & $\mathrm{Z}$ \\
\hline 1 & 6 & 0 & -2.167479 & 0.258645 & 0.000223 \\
\hline 2 & 6 & 0 & -1.272391 & 1.331189 & 0.000074 \\
\hline 3 & 6 & 0 & -1.702531 & -1.051771 & 0.000236 \\
\hline 4 & 1 & 0 & -1.648694 & 2.345341 & 0.000066 \\
\hline 5 & 1 & 0 & -2.401760 & -1.876492 & 0.000352 \\
\hline 6 & 6 & 0 & 0.090562 & 1.096912 & -0.000063 \\
\hline 7 & 6 & 0 & -0.335418 & -1.293541 & 0.000099 \\
\hline 8 & 1 & 0 & 0.810142 & 1.903209 & -0.000180 \\
\hline 9 & 1 & 0 & 0.067706 & -2.297283 & 0.000103 \\
\hline 10 & 6 & 0 & 0.551628 & -0.220893 & -0.000049 \\
\hline 11 & 1 & 0 & -3.232217 & 0.450847 & 0.000330 \\
\hline 12 & 7 & 0 & 1.947072 & -0.578815 & -0.000184 \\
\hline 13 & 8 & 0 & 2.723637 & 0.350355 & -0.000313 \\
\hline
\end{tabular}

FREQUENCIES AND ROTATIONAL CONSTANTS

INDEX NO. DOF TYPE CM-1(UNSCALED) CM-1(SCALED BY) SYMMETRY IR-INTENSITY

$\begin{array}{lrrrr}\text { vib } & 111.99 & 0.00 & \mathrm{~A} & 0.1284 \\ \text { vib } & 246.90 & 0.00 & \mathrm{~A} & 0.2012 \\ \text { vib } & 258.80 & 0.00 & \mathrm{~A} & 2.0106 \\ \text { vib } & 422.34 & 0.00 & \mathrm{~A} & 0.0008 \\ \text { vib } & 448.41 & 0.00 & \mathrm{~A} & 1.2404 \\ \text { vib } & 477.33 & 0.00 & \mathrm{~A} & 2.3959 \\ \text { vib } & 627.58 & 0.00 & \mathrm{~A} & 0.1479 \\ \text { vib } & 684.07 & 0.00 & \mathrm{~A} & 10.2379 \\ \text { vib } & 703.86 & 0.00 & \mathrm{~A} & 28.2879 \\ \text { vib } & 787.60 & 0.00 & \mathrm{~A} & 48.5660 \\ \text { vib } & 831.43 & 0.00 & \mathrm{~A} & 38.7413 \\ \text { vib } & 872.66 & 0.00 & \mathrm{~A} & 0.0022 \\ \text { vib } & 976.53 & 0.00 & \mathrm{~A} & 4.0000 \\ \text { vib } & 1014.72 & 0.00 & \mathrm{~A} & 0.0014 \\ \text { vib } & 1021.70 & 0.00 & \mathrm{~A} & 2.3168 \\ \text { vib } & 1030.90 & 0.00 & \mathrm{~A} & 0.1897 \\ \text { vib } & 1039.31 & 0.00 & \mathrm{~A} & 6.8624 \\ \text { vib } & 1098.72 & 0.00 & \mathrm{~A} & 5.3731 \\ \text { vib } & 1127.84 & 0.00 & \mathrm{~A} & 144.5560 \\ \text { vib } & 1185.84 & 0.00 & \mathrm{~A} & 1.3313 \\ \text { vib } & 1199.85 & 0.00 & \mathrm{~A} & 11.5400 \\ \text { vib } & 1344.86 & 0.00 & \mathrm{~A} & 8.8382 \\ \text { vib } & 1357.03 & 0.00 & \mathrm{~A} & 13.0801\end{array}$




$\begin{array}{rrrrrr}24 & \text { vib } & 1487.85 & 0.00 & \mathrm{~A} & 38.0410 \\ 25 & \text { vib } & 1505.45 & 0.00 & \mathrm{~A} & 9.7816 \\ 26 & \text { vib } & 1582.25 & 0.00 & \mathrm{~A} & 176.6311 \\ 27 & \text { vib } & 1635.76 & 0.00 & \mathrm{~A} & 2.6264 \\ 28 & \text { vib } & 1648.55 & 0.00 & \mathrm{~A} & 10.4728 \\ 29 & \text { vib } & 3169.11 & 0.00 & \mathrm{~A} & 0.5812 \\ 30 & \text { vib } & 3180.32 & 0.00 & \mathrm{~A} & 6.7697 \\ 31 & \text { vib } & 3187.30 & 0.00 & \mathrm{~A} & 7.9442 \\ 32 & \text { vib } & 3197.05 & 0.00 & \mathrm{~A} & 9.6890 \\ 33 & \text { vib } & 3202.79 & 0.00 & \mathrm{~A} & 5.6415 \\ 34 & \text { rot } & 0.1767737 & - & & \\ 35 & \text { rot } & 0.0549466 & - & & \\ 36 & \text { rot } & 0.0419174 & - & & \\ -------------------------------1\end{array}$

\section{Nitrosylhydride.log}

Charge $=0$ Multiplicity $=1$ Stoichiometry HNO

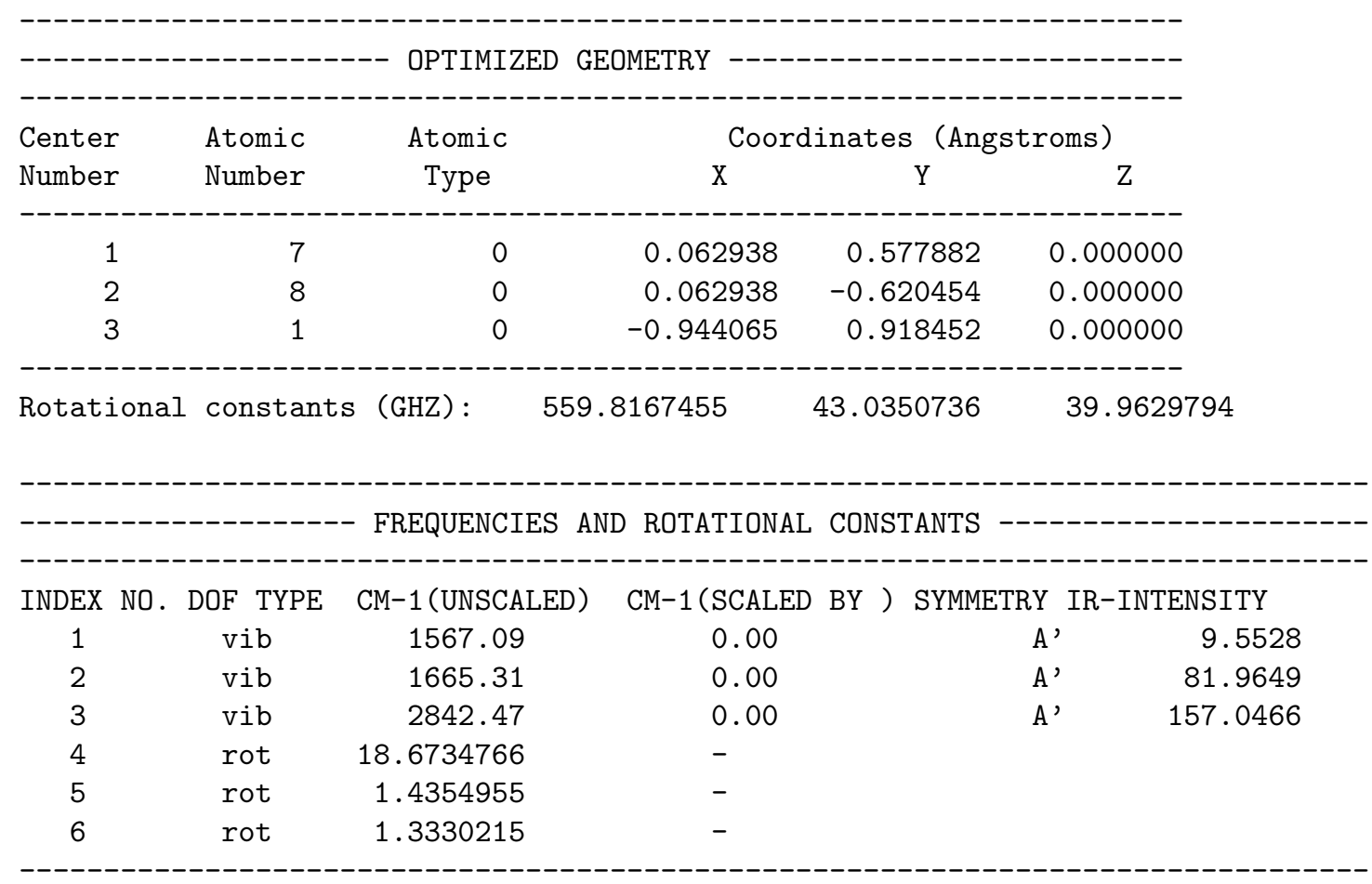

\section{NitrousAcidCis.log}

Charge $=0$ Multiplicity $=1$ Stoichiometry HNO2

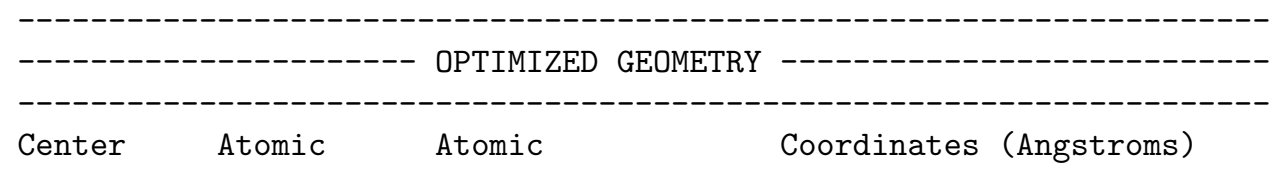




\begin{tabular}{|c|c|c|c|c|c|}
\hline Number & Number & Type & $\mathrm{X}$ & $\mathrm{Y}$ & Z \\
\hline 1 & 7 & 0 & -0.171256 & -0.519382 & -0.000001 \\
\hline 2 & 8 & 0 & -1.056055 & 0.257651 & 0.000000 \\
\hline 3 & 8 & 0 & 1.089193 & 0.067566 & 0.000001 \\
\hline 4 & 1 & 0 & 0.933692 & 1.033936 & 0.000003 \\
\hline
\end{tabular}

FREQUENCIES AND ROTATIONAL CONSTANTS

INDEX NO. DOF TYPE CM-1(UNSCALED) CM-1(SCALED BY) SYMMETRY IR-INTENSITY

$\begin{array}{rrrrrr}1 & \text { vib } & 631.65 & 0.00 & \mathrm{~A} & 30.8705 \\ 2 & \text { vib } & 702.62 & 0.00 & \mathrm{~A} & 99.5158 \\ 3 & \text { vib } & 879.27 & 0.00 & \mathrm{~A} & 279.5728 \\ 4 & \text { vib } & 1337.25 & 0.00 & \mathrm{~A} & 7.2354 \\ 5 & \text { vib } & 1714.95 & 0.00 & \mathrm{~A} & 192.3417 \\ 6 & \text { vib } & 3583.59 & 0.00 & \mathrm{~A} & 19.2885 \\ 7 & \text { rot } & 2.8392069 & - & & \\ 8 & \text { rot } & 0.4422893 & - & & \\ 9 & \text { rot } & 0.3826763 & - & \end{array}$

\section{NitrousAcidTrans.log}

Charge $=0$ Multiplicity $=1$ Stoichiometry HNO2

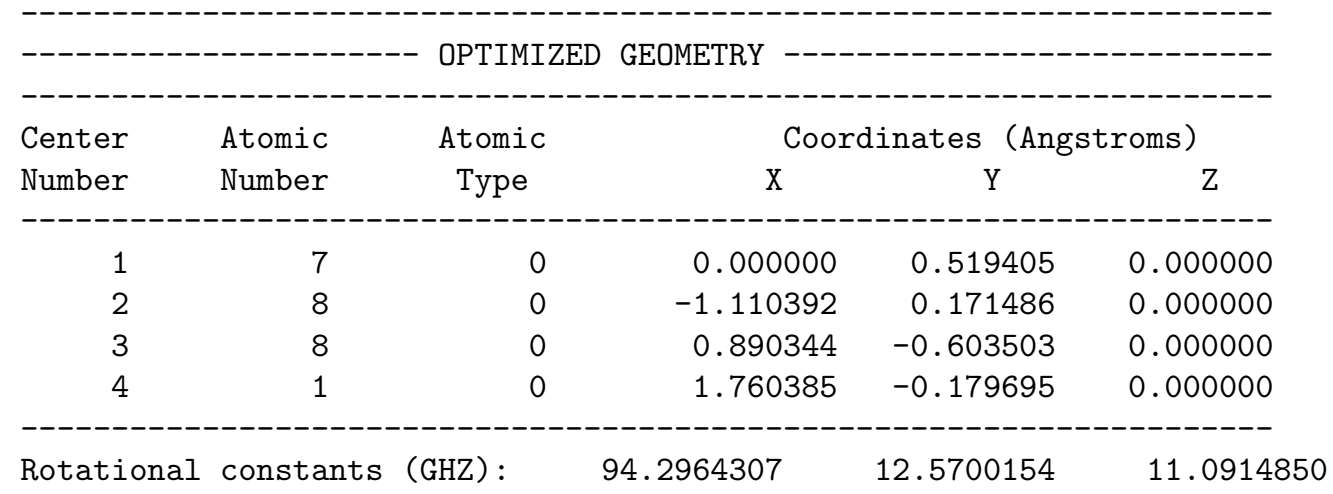

\section{FREQUENCIES AND ROTATIONAL CONSTANTS}

INDEX NO. DOF TYPE CM-1(UNSCALED) CM-1(SCALED BY) SYMMETRY IR-INTENSITY

$\begin{array}{rrrrrr}1 & \text { vib } & 591.19 & 0.00 & \text { A" } & 96.6402 \\ 2 & \text { vib } & 616.86 & 0.00 & \text { A } & 127.1589 \\ 3 & \text { vib } & 826.40 & 0.00 & \text { A } & 132.4632 \\ 4 & \text { vib } & 1302.21 & 0.00 & A & 182.9709 \\ 5 & \text { vib } & 1783.72 & 0.00 & \text { A } & 165.4425 \\ 6 & \text { vib } & 3762.37 & 0.00 & \text { A } & 71.6826 \\ 7 & \text { rot } & 3.1453904 & - & & \\ 8 & \text { rot } & 0.4192906 & - & \end{array}$




\section{NO-freqs.log}

1) LEVEL OF THEORY: UB3LYP/CC-PVTZ+D

Charge $=0$ Multiplicity $=2$ Stoichiometry $\quad$ NO(2)

\begin{tabular}{|c|c|c|c|c|c|}
\hline \multirow{2}{*}{$\begin{array}{l}\text { Center } \\
\text { Number }\end{array}$} & \multirow{2}{*}{$\begin{array}{l}\text { Atomic } \\
\text { Number }\end{array}$} & \multirow{2}{*}{$\begin{array}{c}\text { Atomic } \\
\text { Type }\end{array}$} & \multicolumn{3}{|c|}{ Coordinates (Angstroms) } \\
\hline & & & $\mathrm{X}$ & $\mathrm{Y}$ & $\mathrm{Z}$ \\
\hline 1 & 8 & 0 & 0.000000 & 0.000000 & 0.534793 \\
\hline 2 & 7 & 0 & 0.000000 & 0.000000 & -0.611192 \\
\hline
\end{tabular}

FREQUENCIES AND ROTATIONAL CONSTANTS

INDEX NO. DOF TYPE CM-1 (UNSCALED) CM-1(SCALED BY) SYMMETRY IR-INTENSITY

$\begin{array}{llllll}1 & \text { vib } & 1976.58 & 0.00 & \text { SG } & 37.5636\end{array}$

2 vib $\quad 0.00 \quad 0.00$

$\begin{array}{llll}3 & \text { vib } & 0.00 & 0.00\end{array}$

4 rot $0.0000000 \quad-$

5 rot 1.7191962 -

6 rot 1.7191962 -

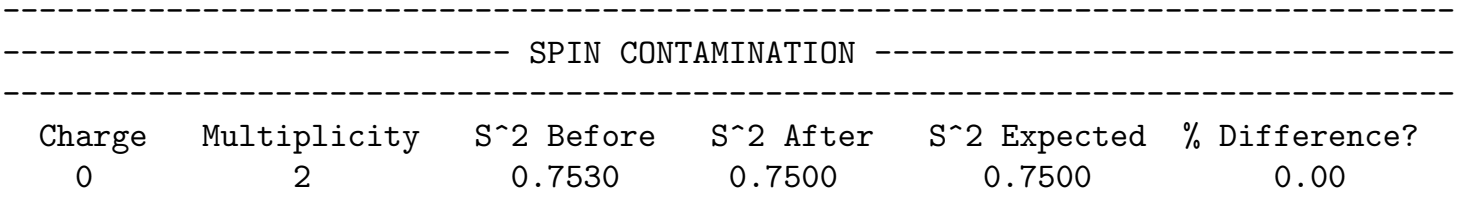

\section{1 nPrOO-freqs.log}

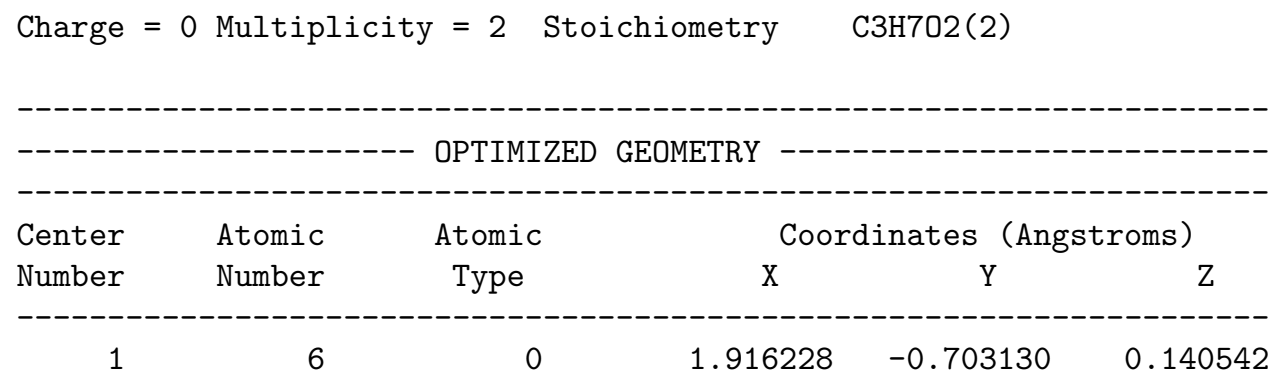

Page 95 of 107 


\begin{tabular}{|c|c|c|c|c|c|}
\hline 2 & 1 & 0 & 1.987587 & -0.802893 & 1.225322 \\
\hline 3 & 1 & 0 & 2.927789 & -0.737143 & -0.264039 \\
\hline 4 & 1 & 0 & 1.374186 & -1.568675 & -0.239333 \\
\hline 5 & 6 & 0 & 1.217998 & 0.599976 & -0.246637 \\
\hline 6 & 1 & 0 & 1.779877 & 1.455965 & 0.135368 \\
\hline 7 & 1 & 0 & 1.192603 & 0.709453 & -1.333321 \\
\hline 8 & 6 & 0 & -0.194759 & 0.718761 & 0.288071 \\
\hline 9 & 1 & 0 & -0.256154 & 0.531604 & 1.360347 \\
\hline 10 & 1 & 0 & -0.644310 & 1.686135 & 0.064535 \\
\hline 11 & 8 & 0 & -1.014270 & -0.293976 & -0.371872 \\
\hline 12 & 8 & 0 & -2.235527 & -0.327035 & 0.116780 \\
\hline
\end{tabular}

FREQUENCIES AND ROTATIONAL CONSTANTS

INDEX NO. DOF TYPE CM-1(UNSCALED) CM-1(SCALED BY ) SYMMETRY IR-INTENSITY

\begin{tabular}{|c|c|c|c|c|}
\hline vib & 59.54 & 0.00 & $\mathrm{~A}$ & 0.5888 \\
\hline vib & 132.95 & 0.00 & $\mathrm{~A}$ & 1.4116 \\
\hline vib & 199.65 & 0.00 & $\mathrm{~A}$ & 0.9628 \\
\hline vib & 296.12 & 0.00 & $\mathrm{~A}$ & 0.5619 \\
\hline vib & 379.28 & 0.00 & $\mathrm{~A}$ & 1.6132 \\
\hline vib & 517.39 & 0.00 & $\mathrm{~A}$ & 7.3869 \\
\hline vib & 750.63 & 0.00 & $\mathrm{~A}$ & 0.6602 \\
\hline vib & 877.80 & 0.00 & $\mathrm{~A}$ & 1.1728 \\
\hline vib & 904.05 & 0.00 & $\mathrm{~A}$ & 13.4932 \\
\hline vib & 924.51 & 0.00 & $\mathrm{~A}$ & 8.7124 \\
\hline vib & 1057.91 & 0.00 & $\mathrm{~A}$ & 3.8038 \\
\hline vib & 1121.91 & 0.00 & $\mathrm{~A}$ & 1.3842 \\
\hline vib & 1155.22 & 0.00 & $\mathrm{~A}$ & 1.8019 \\
\hline vib & 1196.39 & 0.00 & $\mathrm{~A}$ & 14.9626 \\
\hline vib & 1248.63 & 0.00 & $\mathrm{~A}$ & 2.0671 \\
\hline vib & 1306.79 & 0.00 & $\mathrm{~A}$ & 1.0993 \\
\hline vib & 1375.68 & 0.00 & $\mathrm{~A}$ & 10.3442 \\
\hline vib & 1394.91 & 0.00 & $\mathrm{~A}$ & 5.0301 \\
\hline vib & 1422.00 & 0.00 & A & 5.7535 \\
\hline vib & 1480.59 & 0.00 & A & 3.4139 \\
\hline vib & 1491.74 & 0.00 & A & 4.9090 \\
\hline vib & 1503.79 & 0.00 & A & 8.4336 \\
\hline vib & 1509.47 & 0.00 & A & 6.4613 \\
\hline vib & 3029.56 & 0.00 & A & 8.2172 \\
\hline vib & 3032.57 & 0.00 & A & 28.5363 \\
\hline vib & 3046.74 & 0.00 & A & 7.9859 \\
\hline vib & 3060.55 & 0.00 & A & 15.1488 \\
\hline vib & 3092.01 & 0.00 & A & 29.5042 \\
\hline vib & 3101.76 & 0.00 & A & 30.2120 \\
\hline vib & 3105.50 & 0.00 & A & 25.7304 \\
\hline rot & 0.4365882 & - & & \\
\hline rot & 0.0900456 & - & & \\
\hline t & 0.0816619 & - & & \\
\hline
\end{tabular}




$\begin{array}{cccccc}\text { Charge } & \text { Multiplicity } & S^{\wedge} 2 \text { Before } & S^{\wedge} 2 \text { After } & S^{\wedge} 2 \text { Expected } & \% \text { Difference? } \\ 0 & 2 & 0.7538 & 0.7500 & 0.7500 & 0.00\end{array}$

\section{2 o-Benzyne.log}

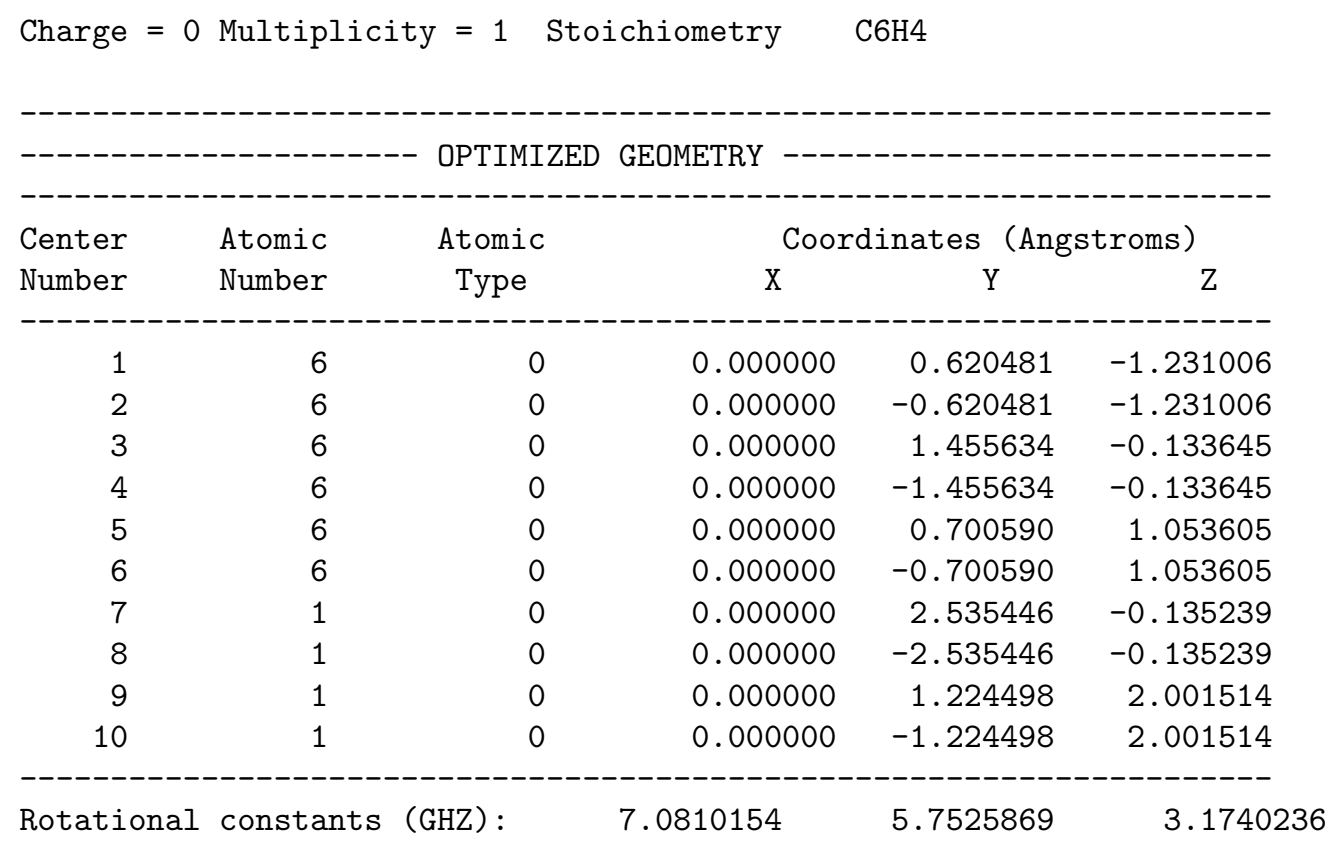

FREQUENCIES AND ROTATIONAL CONSTANTS

\begin{tabular}{|c|c|c|c|c|c|}
\hline INDEX NO. & DOF TYPE & CM-1 (UNSCALED) & CM-1 (SCALED BY ) & SYMMETRY & IR-INTENSITY \\
\hline 1 & vib & 396.81 & 0.00 & B1 & 5.9136 \\
\hline 2 & vib & 427.74 & 0.00 & B2 & 112.1904 \\
\hline 3 & vib & 455.27 & 0.00 & $\mathrm{~A} 2$ & 0.0000 \\
\hline 4 & vib & 610.05 & 0.00 & A2 & 0.0000 \\
\hline 5 & vib & 626.73 & 0.00 & $\mathrm{~A} 1$ & 0.4403 \\
\hline 6 & vib & 753.69 & 0.00 & B1 & 76.5646 \\
\hline 7 & vib & 851.81 & 0.00 & B2 & 31.6627 \\
\hline 8 & vib & 874.68 & 0.00 & $\mathrm{~A} 2$ & 0.0000 \\
\hline 9 & vib & 928.85 & 0.00 & B1 & 0.0037 \\
\hline 10 & vib & 979.49 & 0.00 & $\mathrm{~A} 2$ & 0.0000 \\
\hline 11 & vib & 1000.00 & 0.00 & $\mathrm{~A} 1$ & 7.0557 \\
\hline 12 & vib & 1076.26 & 0.00 & $\mathrm{~A} 1$ & 21.1553 \\
\hline 13 & vib & 1109.58 & 0.00 & B2 & 1.2637 \\
\hline 14 & vib & 1163.10 & 0.00 & A1 & 0.0006 \\
\hline 15 & vib & 1275.68 & 0.00 & B2 & 0.0417 \\
\hline 16 & vib & 1308.15 & 0.00 & A1 & 1.6031 \\
\hline 17 & vib & 1427.37 & 0.00 & B2 & 2.2997 \\
\hline 18 & vib & 1469.11 & 0.00 & B2 & 15.0274 \\
\hline 19 & vib & 1486.24 & 0.00 & A1 & 0.4502 \\
\hline 20 & vib & 2016.55 & 0.00 & $\mathrm{~A} 1$ & 0.0000 \\
\hline 21 & vib & 3160.54 & 0.00 & B2 & 1.1562 \\
\hline 22 & vib & 3176.09 & 0.00 & A1 & 4.6909 \\
\hline
\end{tabular}




\begin{tabular}{lllllr}
23 & vib & 3199.24 & 0.00 & B2 & 26.5840 \\
24 & vib & 3202.69 & 0.00 & A1 & 6.6098 \\
25 & rot & 0.2361972 & - & & \\
26 & rot & 0.1918856 & - & \\
27 & rot & 0.1058740 & - & \\
\hline
\end{tabular}

\section{OH-freqs.log}

Charge $=0$ Multiplicity $=2$ Stoichiometry HO(2)

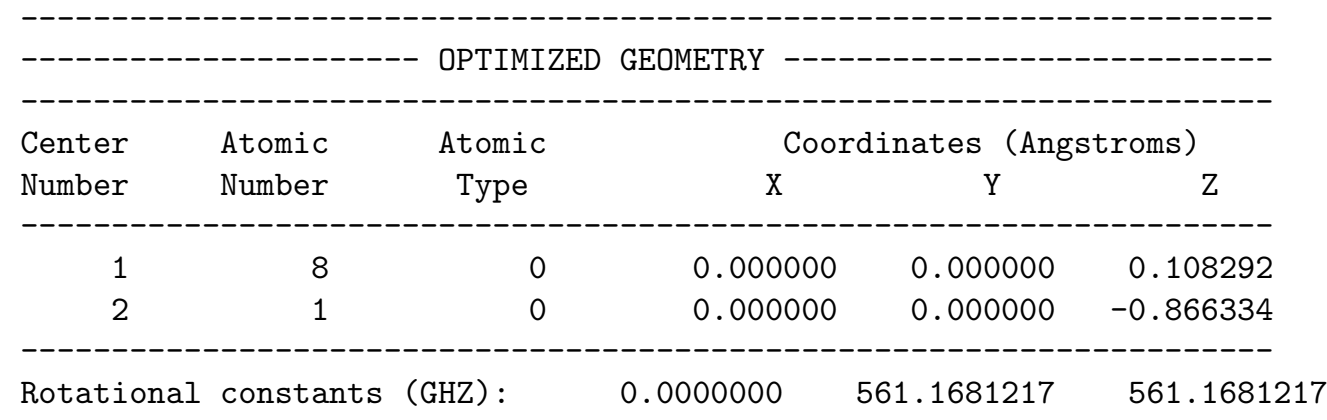

FREQUENCIES AND ROTATIONAL CONSTANTS

\begin{tabular}{|c|c|c|c|c|c|}
\hline INDEX & NO. DOF TYPE & CM-1 (UNSCALED) & CM-1 (SCALED BY 1) & SYMMETRY & IR-INTENSITY \\
\hline 1 & vib & 3698.25 & 3698.25 & SG & 8.6583 \\
\hline 2 & vib & 0.00 & 0.00 & & \\
\hline 3 & vib & 0.00 & 0.00 & & \\
\hline 4 & rot & 0.0000000 & - & & \\
\hline 5 & rot & 18.7185537 & - & & \\
\hline 6 & rot & 18.7185537 & - & & \\
\hline
\end{tabular}

\begin{tabular}{|c|c|c|c|c|c|}
\hline Charge & Multiplicity & $\mathrm{S}^{\wedge} 2$ Before & $\mathrm{S}^{\wedge} 2$ After & $\mathrm{S}^{\wedge} 2$ Expected & $\%$ Difference? \\
\hline 0 & 2 & 0.7522 & 0.7500 & 0.7500 & 0.00 \\
\hline
\end{tabular}

\section{Oxoethenyl.log}

Charge $=0$ Multiplicity $=2$ Stoichiometry $\mathrm{C} 2 \mathrm{HO}(2)$

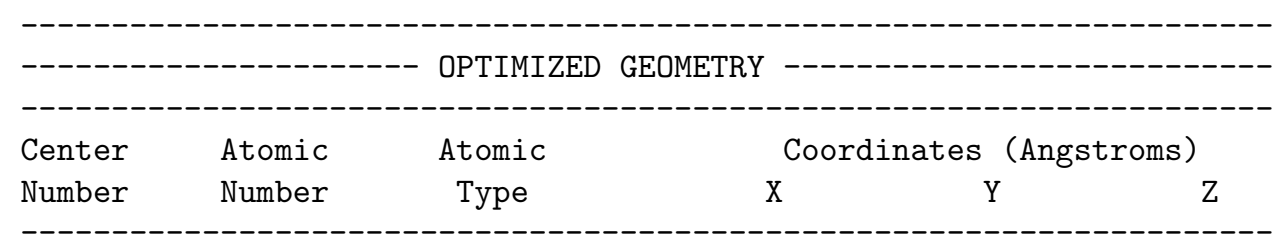




\begin{tabular}{ccrrrr}
1 & 6 & 0 & 1.060510 & -0.684176 & 0.000000 \\
2 & 1 & 0 & 2.119180 & -0.527679 & 0.000000 \\
3 & 6 & 0 & 0.000000 & 0.045527 & 0.000000 \\
4 & 8 & 0 & -1.060280 & 0.544947 & 0.000000 \\
\hline Rotational constants (GHZ) $:$ & 1165.4029239 & 10.9558441 & 10.8538085
\end{tabular}

FREQUENCIES AND ROTATIONAL CONSTANTS

$\begin{array}{ccccrr}\text { INDEX NO. DOF TYPE } & \text { CM-1(UNSCALED) } & \text { CM-1(SCALED BY ) } & \text { SYMMETRY IR-INTENSITY } \\ 1 & \text { vib } & 476.88 & 0.00 & \text { A' } & 225.6343 \\ 2 & \text { vib } & 503.49 & 0.00 & A^{\prime \prime} & 3.2800 \\ 3 & \text { vib } & 566.47 & 0.00 & \text { A } & 11.2617 \\ 4 & \text { vib } & 1264.53 & 0.00 & \text { A } & 6.6778 \\ 5 & \text { vib } & 2083.18 & 0.00 & \text { A } & 309.0501 \\ 6 & \text { vib } & 3343.03 & 0.00 & \text { A' } \\ 7 & \text { rot } & 38.8736572 & - & & 43.3751 \\ 8 & \text { rot } & 0.3654476 & - & & \\ 9 & \text { rot } & 0.3620441 & - & \end{array}$

SPIN CONTAMINATION

$\begin{array}{cccccc}\text { Charge } & \text { Multiplicity } & S^{\wedge} 2 \text { Before } & S^{\wedge} 2 \text { After } & S^{\wedge} 2 \text { Expected } & \% \text { Difference? } \\ 0 & 2 & 0.7578 & 0.7500 & 0.7500 & 0.00\end{array}$

\section{Peroxynitrousacid-cis-cis.log}

Charge $=0$ Multiplicity $=1$ Stoichiometry HNO3

\begin{tabular}{|c|c|c|c|c|c|}
\hline \multirow{2}{*}{$\begin{array}{l}\text { Center } \\
\text { Number }\end{array}$} & \multirow{2}{*}{$\begin{array}{l}\text { Atomic } \\
\text { Number }\end{array}$} & \multirow{2}{*}{$\begin{array}{c}\text { Atomic } \\
\text { Type }\end{array}$} & \multicolumn{3}{|c|}{ Coordinates (Angstroms) } \\
\hline & & & $\mathrm{X}$ & Y & Z \\
\hline 1 & 7 & 0 & 0.836426 & 0.539645 & -0.000001 \\
\hline 2 & 8 & 0 & -0.533370 & 0.735466 & 0.000000 \\
\hline 3 & 8 & 0 & 1.172568 & -0.594197 & 0.000000 \\
\hline 4 & 8 & 0 & -1.297847 & -0.470049 & 0.000000 \\
\hline 5 & 1 & 0 & -0.585792 & -1.147273 & 0.000000 \\
\hline
\end{tabular}

FREQUENCIES AND ROTATIONAL CONSTANTS

INDEX NO. DOF TYPE CM-1(UNSCALED) CM-1(SCALED BY ) SYMMETRY IR-INTENSITY

\begin{tabular}{|c|c|c|c|}
\hline & vib & 373.40 & 0.00 \\
\hline & & 30 & 0.00 \\
\hline
\end{tabular}




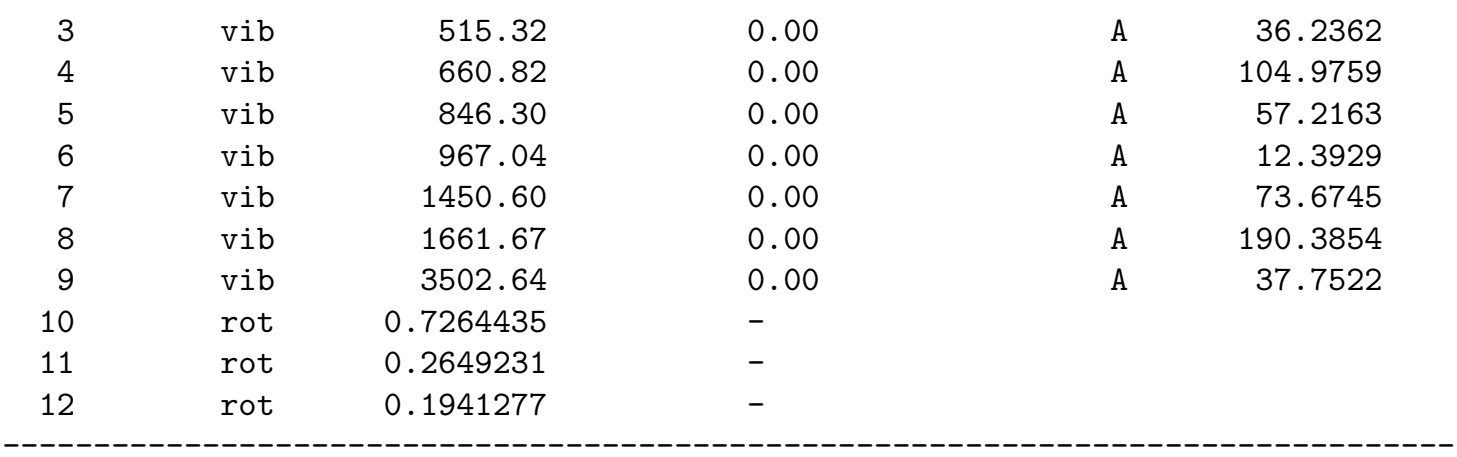

\section{Peroxynitrousacid-cis-perp.log}

Charge $=0$ Multiplicity $=1$ Stoichiometry HNO3

\begin{tabular}{|c|c|c|c|c|c|}
\hline \multirow{2}{*}{$\begin{array}{l}\text { Center } \\
\text { Number }\end{array}$} & \multirow{2}{*}{$\begin{array}{l}\text { Atomic } \\
\text { Number }\end{array}$} & \multirow{2}{*}{$\begin{array}{c}\text { Atomic } \\
\text { Type }\end{array}$} & \multicolumn{3}{|c|}{ Coordinates (Angstroms) } \\
\hline & & & $\mathrm{X}$ & $\mathrm{Y}$ & Z \\
\hline 1 & 7 & 0 & -0.903080 & 0.506160 & 0.006640 \\
\hline 2 & 8 & 0 & 0.549228 & 0.735260 & 0.019892 \\
\hline 3 & 8 & 0 & -1.218721 & -0.606977 & -0.008047 \\
\hline 4 & 8 & 0 & 1.289399 & -0.473418 & -0.117487 \\
\hline 5 & 1 & 0 & 1.362313 & -0.782030 & 0.798653 \\
\hline
\end{tabular}

FREQUENCIES AND ROTATIONAL CONSTANTS

INDEX NO. DOF TYPE CM-1(UNSCALED) CM-1(SCALED BY) SYMMETRY IR-INTENSITY

\begin{tabular}{|c|c|c|c|c|c|}
\hline 1 & vib & 150.33 & 0.00 & A & 61.0359 \\
\hline 2 & vib & 322.04 & 0.00 & A & 0.9806 \\
\hline 3 & vib & 389.58 & 0.00 & A & 2.8420 \\
\hline 4 & vib & 477.93 & 0.00 & A & 177.5880 \\
\hline 5 & vib & 831.04 & 0.00 & A & 45.3375 \\
\hline 6 & vib & 965.77 & 0.00 & A & 3.3925 \\
\hline 7 & vib & 1386.90 & 0.00 & A & 47.8077 \\
\hline 8 & vib & 1787.08 & 0.00 & A & 231.6795 \\
\hline 9 & vib & 3722.85 & 0.00 & A & 44.8243 \\
\hline 10 & rot & 0.7285524 & - & & \\
\hline 11 & rot & 0.2432486 & - & & \\
\hline 12 & rot & 0.1856736 & - & & \\
\hline
\end{tabular}

\section{Peroxynitrousacid-trans-perp.log}

Charge $=0$ Multiplicity $=1$ Stoichiometry HNO3 


\begin{tabular}{|c|c|c|c|c|c|c|}
\hline Center & Atomic & Atomic & \multicolumn{4}{|c|}{ Coordinates (Angstroms) } \\
\hline Number & Number & Type & $\mathrm{X}$ & $\mathrm{Y}$ & \multicolumn{2}{|r|}{$\mathrm{Z}$} \\
\hline 1 & 7 & 0 & -0.678783 & -0.466235 & \multicolumn{2}{|c|}{-0.002420} \\
\hline 2 & 8 & 0 & 0.415113 & 0.537436 & \multicolumn{2}{|c|}{-0.000917} \\
\hline 3 & 8 & 0 & -1.696514 & 0.084213 & \multicolumn{2}{|c|}{0.010800} \\
\hline 4 & 8 & 0 & 1.634184 & -0.186826 & \multicolumn{2}{|c|}{-0.109298} \\
\hline 5 & 1 & 0 & 1.929210 & -0.214940 & \multicolumn{2}{|c|}{0.812261} \\
\hline \multicolumn{3}{|c|}{ Rotational constants (GHZ): } & 54.9173834 & 4.9306682 & \multicolumn{2}{|r|}{4.5917474} \\
\hline \multicolumn{7}{|c|}{$\begin{array}{l}-1 \\
-1\end{array}$} \\
\hline \multirow{2}{*}{\multicolumn{7}{|c|}{ INDEX NO. DOF TYPE CM-1 (UNSCALED) CM-1 (SCALED BY ) SYMMETRY IR-INTENSITY }} \\
\hline & & & & & & \\
\hline 2 & vib & 290.26 & 0.00 & & A & 107.6613 \\
\hline 3 & vib & 357.09 & 0.00 & & A & 7.1812 \\
\hline 4 & vib & 468.25 & 0.00 & & A & 95.8893 \\
\hline 5 & vib & 812.92 & 0.00 & & $\mathrm{~A}$ & 121.6247 \\
\hline 6 & vib & 1017.63 & 0.00 & & A & 70.2398 \\
\hline 7 & vib & 1409.21 & 0.00 & & A & 56.3864 \\
\hline 8 & vib & 1803.40 & 0.00 & & A & 240.2073 \\
\hline 9 & vib & 3739.18 & 0.00 & & A & 49.4997 \\
\hline 10 & rot & 1.8318467 & - & & & \\
\hline 11 & rot & 0.1644694 & - & & & \\
\hline 12 & rot & 0.1531642 & - & & & \\
\hline
\end{tabular}

\section{Toluene.log}

Charge $=0$ Multiplicity $=1$ Stoichiometry $\quad$ C7H8

\begin{tabular}{|c|c|c|c|c|c|}
\hline \multirow{2}{*}{$\begin{array}{l}\text { Center } \\
\text { Number }\end{array}$} & \multirow{2}{*}{$\begin{array}{l}\text { Atomic } \\
\text { Number }\end{array}$} & \multirow{2}{*}{$\begin{array}{c}\text { Atomic } \\
\text { Type }\end{array}$} & \multicolumn{3}{|c|}{ Coordinates (Angstroms) } \\
\hline & & & $\mathrm{X}$ & Y & Z \\
\hline 1 & 6 & 0 & 0.007143 & -1.196413 & 1.200096 \\
\hline 2 & 6 & 0 & 0.007143 & 0.193393 & 1.197187 \\
\hline 3 & 6 & 0 & 0.004123 & 0.910508 & 0.000000 \\
\hline 4 & 6 & 0 & 0.007143 & 0.193393 & -1.197187 \\
\hline 5 & 6 & 0 & 0.007143 & -1.196413 & -1.200096 \\
\hline 6 & 6 & 0 & 0.006145 & -1.897576 & 0.000000 \\
\hline 7 & 1 & 0 & 0.011748 & -1.731637 & 2.140684 \\
\hline 8 & 1 & 0 & 0.011968 & 0.730042 & 2.138177 \\
\hline 9 & 1 & 0 & 0.011968 & 0.730042 & -2.138177 \\
\hline 10 & 1 & 0 & 0.011748 & -1.731637 & -2.140684 \\
\hline
\end{tabular}




$\begin{array}{ccccrr}11 & 1 & 0 & 0.009071 & -2.979355 & 0.000000 \\ 12 & 6 & 0 & -0.027320 & 2.416962 & 0.000000 \\ 13 & 1 & 0 & 0.464996 & 2.825623 & 0.882553 \\ 14 & 1 & 0 & 0.464996 & 2.825623 & -0.882553 \\ 15 & 1 & 0 & -1.055615 & 2.788171 & 0.000000 \\ -15 & & 0 & & & \end{array}$

FREQUENCIES AND ROTATIONAL CONSTANTS

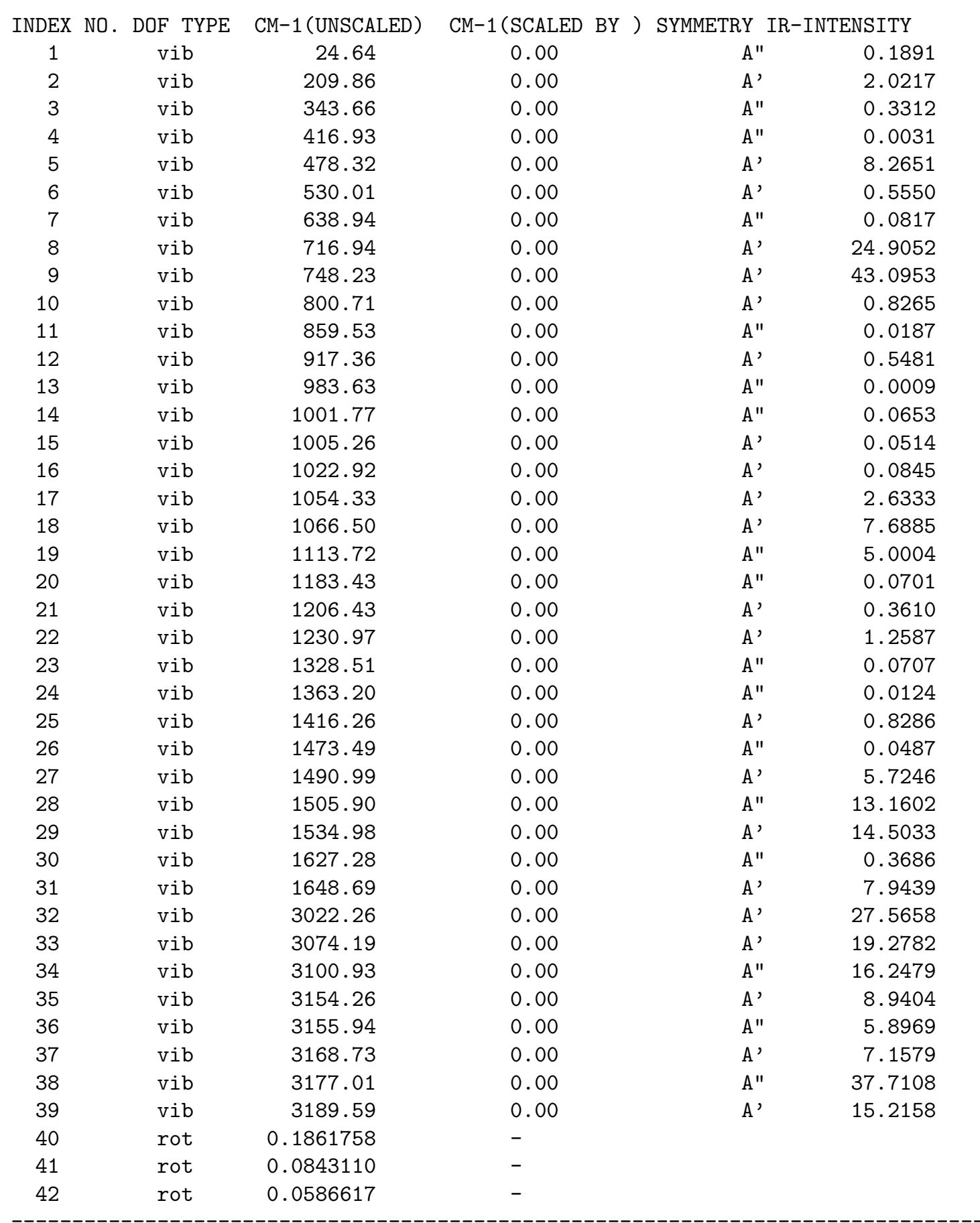




\section{Trans-2-butene.log}

\begin{tabular}{|c|c|c|c|c|c|}
\hline \multirow{2}{*}{$\begin{array}{l}\text { Center } \\
\text { Number }\end{array}$} & \multirow{2}{*}{$\begin{array}{l}\text { Atomic } \\
\text { Number }\end{array}$} & \multirow{2}{*}{$\begin{array}{l}\text { Atomic } \\
\text { Type }\end{array}$} & \multicolumn{3}{|c|}{ Coordinates (Angstroms) } \\
\hline & & & $\mathrm{X}$ & $\mathrm{Y}$ & Z \\
\hline 1 & 6 & 0 & -0.465946 & -0.462433 & -0.100814 \\
\hline 2 & 1 & 0 & -1.511218 & -0.165088 & -0.058190 \\
\hline 3 & 6 & 0 & 0.465946 & 0.462433 & 0.100814 \\
\hline 4 & 1 & 0 & 1.511218 & 0.165088 & 0.058190 \\
\hline 5 & 6 & 0 & -0.203557 & -1.908677 & -0.387824 \\
\hline 6 & 1 & 0 & 0.865135 & -2.123165 & -0.415828 \\
\hline 7 & 1 & 0 & -0.658498 & -2.551985 & 0.370429 \\
\hline 8 & 1 & 0 & -0.634139 & -2.206657 & -1.347772 \\
\hline 9 & 6 & 0 & 0.203557 & 1.908677 & 0.387824 \\
\hline 10 & 1 & 0 & 0.634139 & 2.206657 & 1.347772 \\
\hline 11 & 1 & 0 & 0.658498 & 2.551985 & -0.370429 \\
\hline 12 & 1 & 0 & -0.865135 & 2.123165 & 0.415828 \\
\hline
\end{tabular}

FREQUENCIES AND ROTATIONAL CONSTANTS

INDEX NO. DOF TYPE CM-1 (UNSCALED) CM-1 (SCALED BY ) SYMMETRY IR-INTENSITY

\begin{tabular}{|c|c|c|c|c|c|}
\hline & vib & 179.50 & 0.00 & $\mathrm{AU}$ & 1.0851 \\
\hline & vib & 230.27 & 0.00 & $\mathrm{AG}$ & 0.0000 \\
\hline & vib & 246.93 & 0.00 & $\mathrm{AU}$ & 3.3792 \\
\hline & vib & 283.04 & 0.00 & $\mathrm{AU}$ & 1.1994 \\
\hline & vib & 502.89 & 0.00 & AG & 0.0000 \\
\hline & vib & 763.85 & 0.00 & $\mathrm{AG}$ & 0.0000 \\
\hline & vib & 875.51 & 0.00 & $\mathrm{AG}$ & 0.0000 \\
\hline & vib & 990.97 & 0.00 & AU & 14.5863 \\
\hline & vib & 1002.65 & 0.00 & AU & 34.1192 \\
\hline & vib & 1071.03 & 0.00 & AU & 1.3370 \\
\hline & vib & 1073.70 & 0.00 & AU & 3.1843 \\
\hline & vib & 1074.22 & 0.00 & $\mathrm{AG}$ & 0.0000 \\
\hline & vib & 1168.51 & 0.00 & $\mathrm{AG}$ & 0.0000 \\
\hline & vib & 1335.82 & 0.00 & AU & 4.9180 \\
\hline & vib & 1338.67 & 0.00 & $\mathrm{AG}$ & 0.0000 \\
\hline & vib & 1415.39 & 0.00 & $\mathrm{AU}$ & 3.5471 \\
\hline & vib & 1416.81 & 0.00 & $A G$ & 0.0000 \\
\hline & vib & 1480.63 & 0.00 & $\mathrm{AU}$ & 12.3924 \\
\hline & vib & 1481.07 & 0.00 & $\mathrm{AG}$ & 0.0000 \\
\hline & vib & 1490.39 & 0.00 & $\mathrm{AG}$ & 0.0000 \\
\hline & vib & 1498.64 & 0.00 & $\mathrm{AU}$ & 18.1803 \\
\hline & vib & 1740.98 & 0.00 & $\mathrm{AG}$ & 0.0000 \\
\hline & vib & 3011.22 & 0.00 & $\mathrm{AU}$ & 70.5937 \\
\hline & vib & 3011.57 & 0.00 & $\mathrm{AG}$ & 0.0000 \\
\hline
\end{tabular}




\begin{tabular}{|c|c|c|c|c|c|}
\hline 25 & vib & 3049.91 & 0.00 & $\mathrm{AU}$ & 47.8786 \\
\hline 26 & vib & 3050.47 & 0.00 & $A G$ & 0.0000 \\
\hline 27 & vib & 3086.23 & 0.00 & $\mathrm{AU}$ & 19.8532 \\
\hline 28 & vib & 3087.79 & 0.00 & AG & 0.0000 \\
\hline 29 & vib & 3108.74 & 0.00 & $\mathrm{AG}$ & 0.0000 \\
\hline 30 & vib & 3117.96 & 0.00 & $\mathrm{AU}$ & 73.1618 \\
\hline 31 & rot & 1.1709801 & - & & \\
\hline 32 & rot & 0.1244132 & - & & \\
\hline 33 & rot & 0.1173104 & - & & \\
\hline
\end{tabular}

\section{Vinoxy-radical-freqs.log}

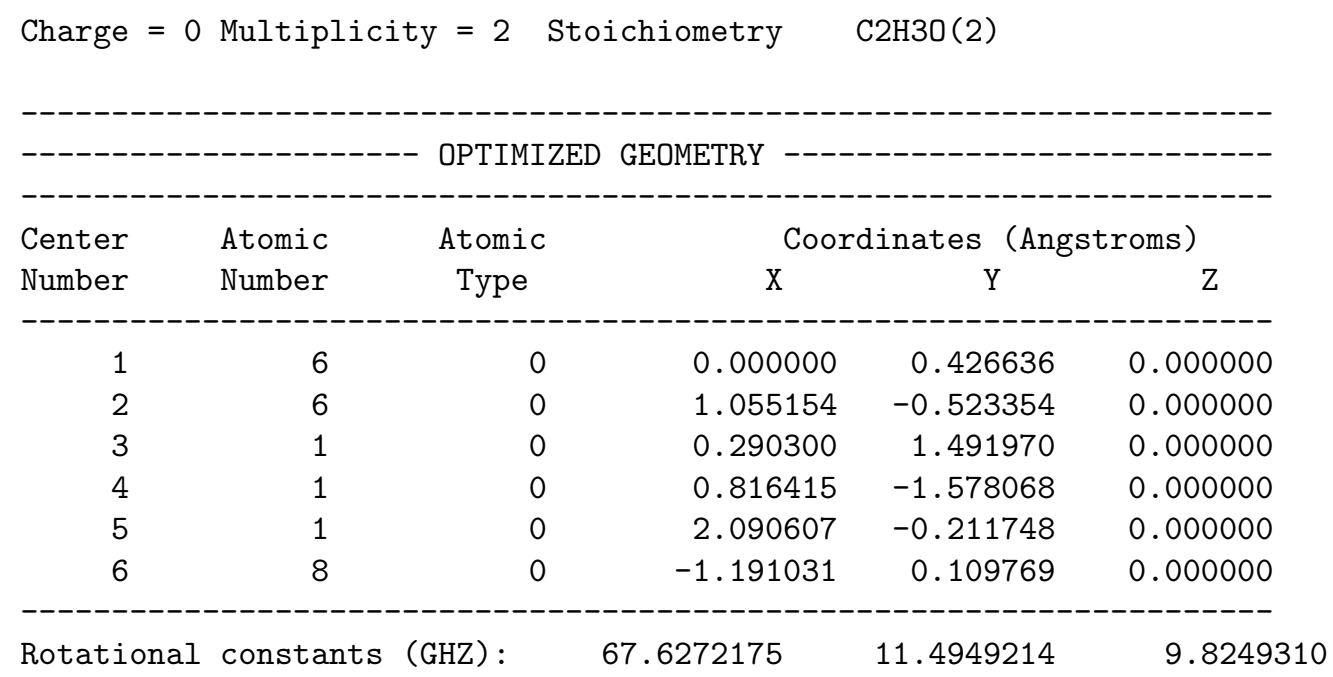

FREQUENCIES AND ROTATIONAL CONSTANTS

INDEX NO. DOF TYPE CM-1 (UNSCALED) CM-1 (SCALED BY) SYMMETRY IR-INTENSITY

\begin{tabular}{|c|c|c|c|c|c|}
\hline 1 & vib & 448.63 & 0.00 & A" & 0.2919 \\
\hline 2 & vib & 506.86 & 0.00 & $A^{\prime}$ & 12.6922 \\
\hline 3 & vib & 764.07 & 0.00 & $A^{\prime \prime}$ & 33.9091 \\
\hline 4 & vib & 978.75 & 0.00 & $A^{\prime}$ & 3.3439 \\
\hline 5 & vib & 982.57 & 0.00 & A" & 0.7748 \\
\hline 6 & vib & 1158.64 & 0.00 & $A^{\prime}$ & 20.7617 \\
\hline 7 & vib & 1399.84 & 0.00 & $A^{\prime}$ & 13.1868 \\
\hline 8 & vib & 1474.56 & 0.00 & $A^{\prime}$ & 12.0741 \\
\hline 9 & vib & 1551.36 & 0.00 & $A^{\prime}$ & 35.9596 \\
\hline 10 & vib & 2933.92 & 0.00 & $A^{\prime}$ & 91.3428 \\
\hline 11 & vib & 3141.01 & 0.00 & $A^{\prime}$ & 2.3671 \\
\hline 12 & vib & 3253.58 & 0.00 & $A^{\prime}$ & 3.1430 \\
\hline 13 & rot & 2.2558012 & - & & \\
\hline 14 & rot & 0.3834293 & - & & \\
\hline 15 & rot & 0.3277244 & - & & \\
\hline
\end{tabular}




\begin{tabular}{|c|c|c|c|c|c|}
\hline Charge & Multiplicity & $S^{\wedge} 2$ Before & $S^{\wedge} 2$ After & $\mathrm{S}^{\wedge} 2$ Expected & $\%$ Difference? \\
\hline 0 & 2 & 0.7679 & 0.7501 & 0.7500 & 0.01 \\
\hline
\end{tabular}

\section{Vinylalcohol-anti.log}

\begin{tabular}{|c|c|c|c|c|c|}
\hline \multirow{2}{*}{$\begin{array}{l}\text { Center } \\
\text { Number }\end{array}$} & \multirow{2}{*}{$\begin{array}{l}\text { Atomic } \\
\text { Number }\end{array}$} & \multirow{2}{*}{$\begin{array}{c}\text { Atomic } \\
\text { Type }\end{array}$} & \multicolumn{3}{|c|}{ Coordinates (Angstroms) } \\
\hline & & & $\mathrm{X}$ & Y & Z \\
\hline 1 & 6 & 0 & 1.234990 & -0.067769 & 0.000000 \\
\hline 2 & 1 & 0 & 1.426624 & -1.131351 & 0.000000 \\
\hline 3 & 1 & 0 & 2.073619 & 0.610339 & 0.000000 \\
\hline 4 & 6 & 0 & 0.000000 & 0.412241 & 0.000000 \\
\hline 5 & 1 & 0 & -0.199141 & 1.478802 & 0.000000 \\
\hline 6 & 8 & 0 & -1.101769 & -0.395828 & 0.000000 \\
\hline 7 & 1 & 0 & -1.896890 & 0.141997 & 0.000000 \\
\hline
\end{tabular}

\section{FREQUENCIES AND ROTATIONAL CONSTANTS}

INDEX NO. DOF TYPE CM-1 (UNSCALED) CM-1 (SCALED BY ) SYMMETRY IR-INTENSITY

\begin{tabular}{|c|c|c|c|c|c|}
\hline 1 & vib & 263.05 & 0.00 & A" & 97.5310 \\
\hline 2 & vib & 484.35 & 0.00 & $A^{\prime}$ & 3.3472 \\
\hline 3 & vib & 717.70 & 0.00 & A" & 18.9699 \\
\hline 4 & vib & 858.92 & 0.00 & A" & 57.2064 \\
\hline 5 & vib & 959.06 & 0.00 & $A^{\prime}$ & 53.2108 \\
\hline 6 & vib & 977.31 & 0.00 & A" & 19.9418 \\
\hline 7 & vib & 1144.66 & 0.00 & $A^{\prime}$ & 14.9356 \\
\hline 8 & vib & 1286.50 & 0.00 & $A^{\prime}$ & 215.3771 \\
\hline 9 & vib & 1351.58 & 0.00 & $A^{\prime}$ & 3.3567 \\
\hline 10 & vib & 1442.58 & 0.00 & $A^{\prime}$ & 0.2734 \\
\hline 11 & vib & 1730.08 & 0.00 & $A^{\prime}$ & 104.4100 \\
\hline 12 & vib & 3139.94 & 0.00 & $A^{\prime}$ & 19.0315 \\
\hline 13 & vib & 3163.48 & 0.00 & $A^{\prime}$ & 0.2708 \\
\hline 14 & vib & 3253.93 & 0.00 & $A^{\prime}$ & 5.7888 \\
\hline 15 & vib & 3852.34 & 0.00 & $A^{\prime}$ & 83.3072 \\
\hline 16 & rot & 2.1523543 & - & & \\
\hline 17 & rot & 0.3486084 & - & & \\
\hline 18 & rot & 0.3000160 & - & & \\
\hline
\end{tabular}

\section{Vinylalcohol-syn.log}

Charge $=0$ Multiplicity $=1$ Stoichiometry $\quad$ C2H40 


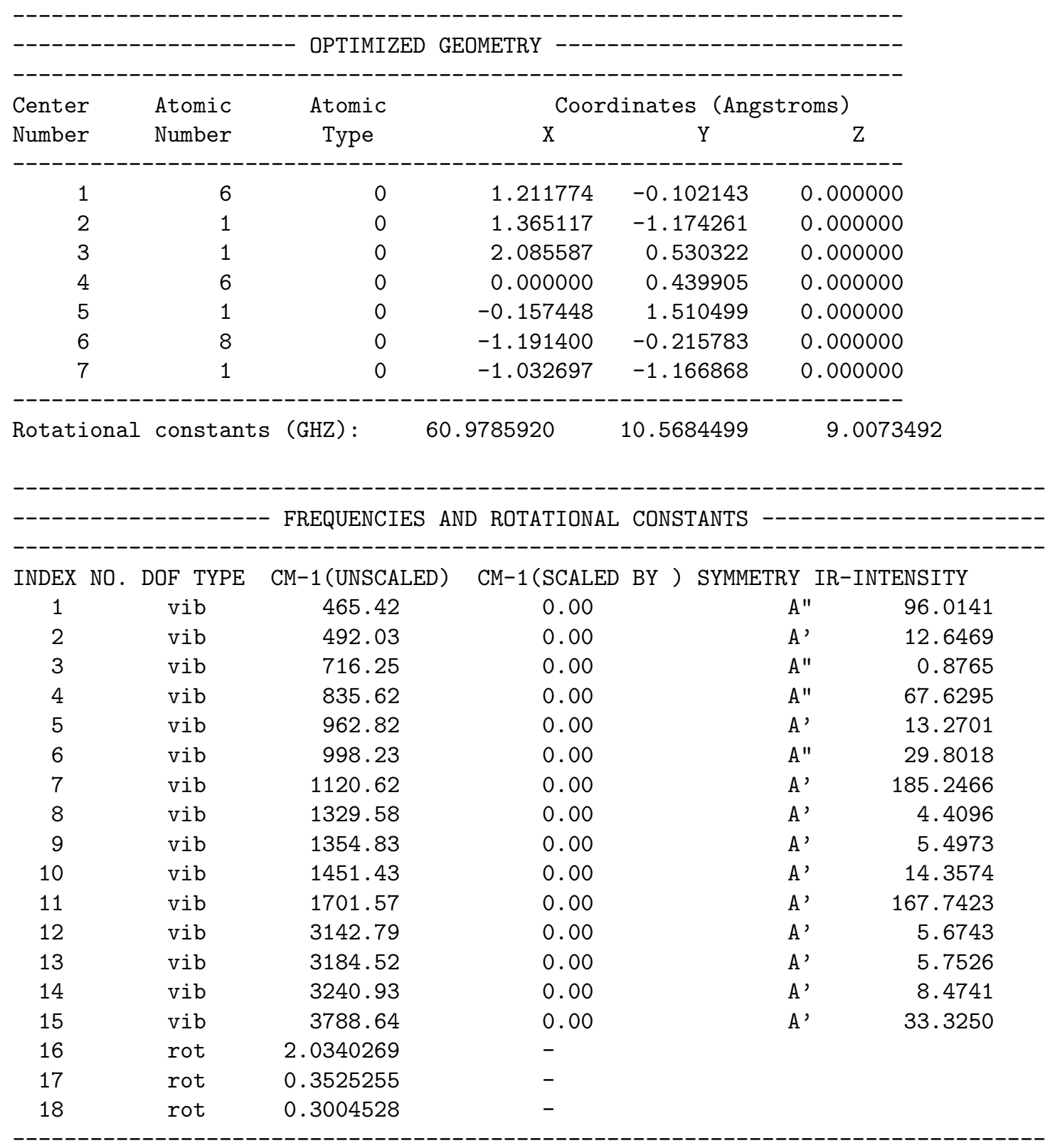

\section{Vinylidene.log}

\begin{tabular}{|c|c|c|c|c|c|}
\hline Center & Atomic & Atomic & Coo: & inates (Ang & troms) \\
\hline Number & Number & Type & $\mathrm{X}$ & $\mathrm{Y}$ & $\mathrm{Z}$ \\
\hline 1 & 6 & 0 & 0.000000 & 0.000000 & 0.816333 \\
\hline 2 & 6 & 0 & 0.000000 & 0.000000 & -0.474647 \\
\hline 3 & 1 & 0 & 0.000000 & 0.936629 & -1.025057 \\
\hline 4 & 1 & 0 & 0.000000 & -0.936629 & -1.025057 \\
\hline
\end{tabular}




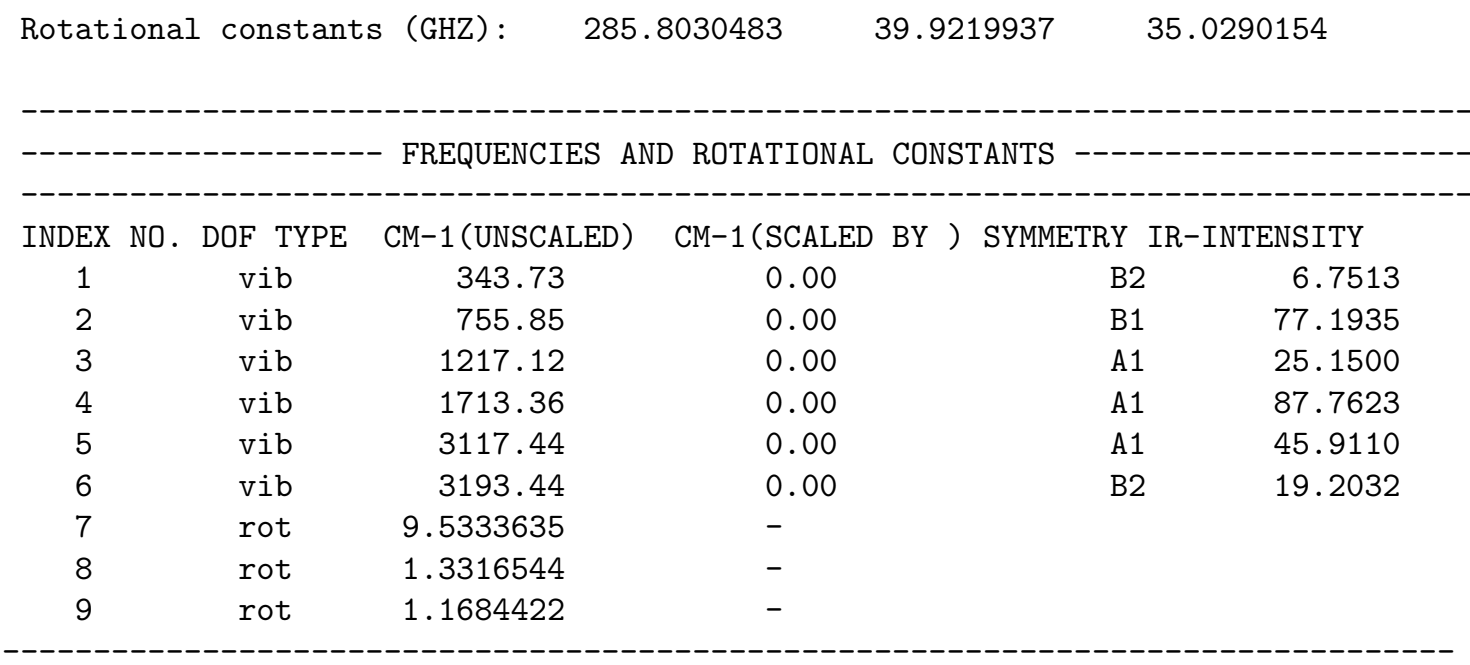

